Energy Systems Environmental Restoration Program

Clinch River Environmental Restoration Program

\title{
Water Quality Monitoring Report for the White Oak Creek Embayment
}

\author{
C. J. Ford \\ M. T. Wefer
}

Date Issued-January 1993

Prepared by

Environmental Sciences Division

Oak Ridge National Laboratory

ESD Publication 4039

Prepared for

U.S. Department of Energy

Office of Environmental Restoration and Waste Management under budget and reporting code EW 20

OAK RIDGE NATIONAL LABORATORY

Oak Ridge, Tennessee 37831-6285

managed by

MARTIN MARIETTA ENERGY SYSTEMS, INC.

for the

U.S. DEPARTMENT OF ENERGY under contract DE-AC05-84OR21400 


\section{Author Affiliations}

C. J. Ford is a member of the Environmental Sciences Division, Oak Ridge National Laboratory, Martin Marietta Energy, Systems, Inc. M. T.

Wefer is employed by Automated Sciences Group, Inc., Oak Ridge, Tennessee. 


\section{CONTENTS}

FIGURES $\ldots \ldots \ldots \ldots \ldots \ldots \ldots \ldots \ldots \ldots \ldots \ldots \ldots \ldots \ldots \ldots \ldots$

TABLES $\ldots \ldots \ldots \ldots \ldots \ldots \ldots \ldots \ldots \ldots \ldots \ldots \ldots \ldots \ldots \ldots \ldots \ldots$

ABBREVIATIONS $\ldots \ldots \ldots \ldots \ldots \ldots \ldots \ldots \ldots \ldots \ldots \ldots \ldots \ldots \ldots \ldots \ldots$

ACKNOWLEDGMENTS $\ldots \ldots \ldots \ldots \ldots \ldots \ldots \ldots \ldots \ldots \ldots \ldots \ldots \ldots \ldots$

EXECUTTVE SUMMARY $\ldots \ldots \ldots \ldots \ldots \ldots \ldots \ldots \ldots \ldots \ldots \ldots \ldots \ldots \ldots \ldots$

1. INTRODUCTION $\ldots \ldots \ldots \ldots \ldots \ldots \ldots \ldots \ldots \ldots \ldots \ldots \ldots \ldots$

1.1 SITE BACKGROUND $\ldots \ldots \ldots \ldots \ldots \ldots \ldots \ldots \ldots \ldots \ldots \ldots$

1.2 WOCE SITE CHARACTERIZATION $\ldots \ldots \ldots \ldots \ldots \ldots \ldots \ldots \ldots \ldots$

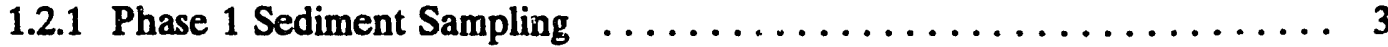

1.2.2 Characterization of WOCE Sediments ................ 3

1.2.3 Characterization of WOCE Surface Water .............. 3

1.3 OBJECTIVES ............................... 3

2. MATERIALS AND METHODS: SAMPLE COLLECTION, ANALYSIS,

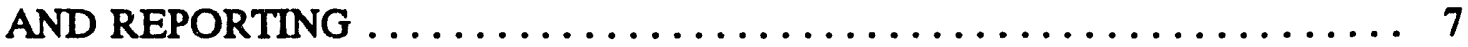

2.1 Monitoring Stations, Strategy, and Methods ............... 7

2.2 Water Quality Monitoring Variables ..................... 9

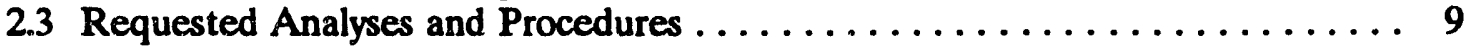

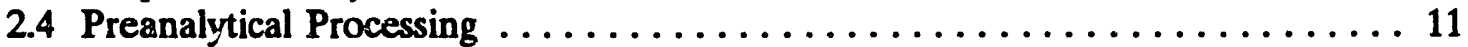

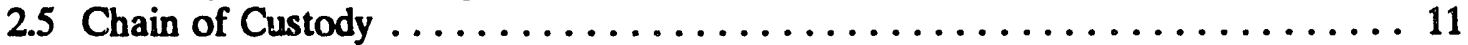

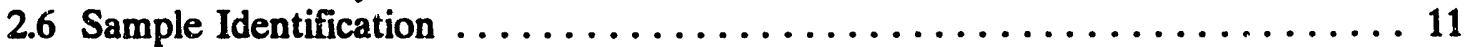

2.7 Quality Assurance/Quality Control ................... 11

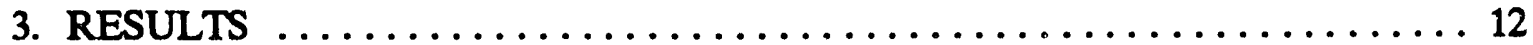

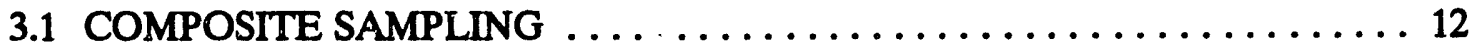

3.2 CONTINUOUS WATER QUALITY MONITORING ............ 23

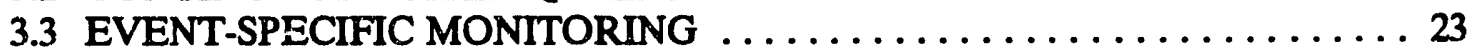

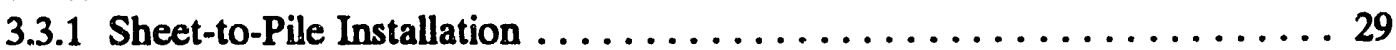

3.3.2 Rock Armoring . . . . . . . . . . . . . . . . . . . . 29

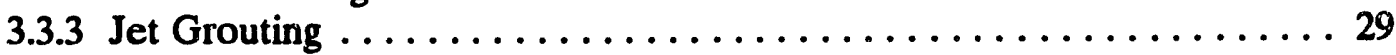

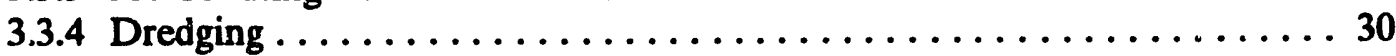

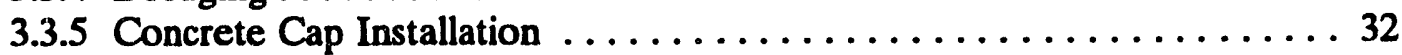

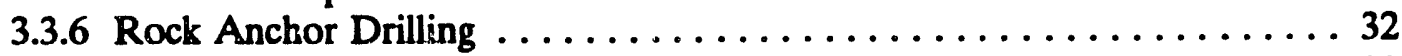

3.3.7 Postconstruction Monitoring .................... 32

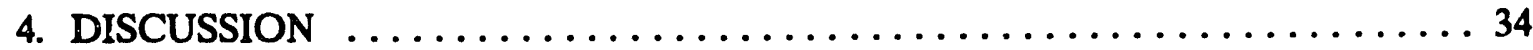

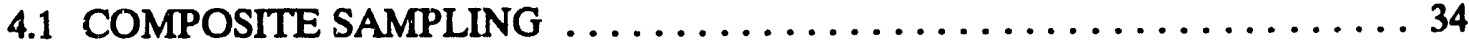

4.2 CONTINUOUS WATER QUALITY MONITORING $\ldots \ldots \ldots \ldots \ldots \ldots . \ldots \ldots$

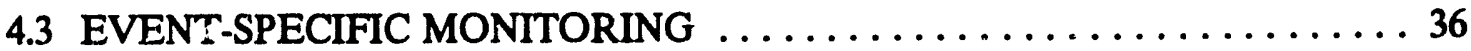

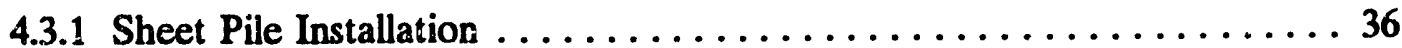




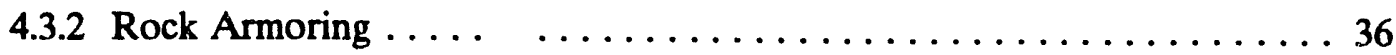

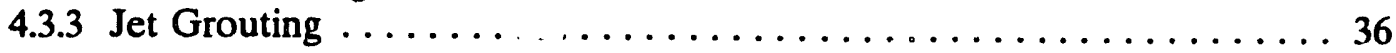

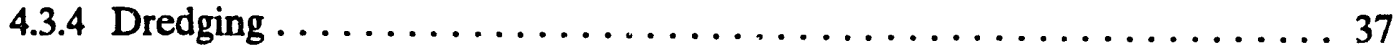

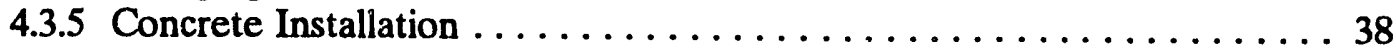

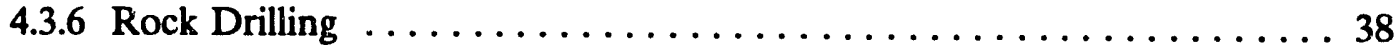

4.3.7 Postconstruction Monitoring $\ldots \ldots \ldots \ldots \ldots \ldots \ldots \ldots \ldots \ldots \ldots \ldots \ldots \ldots$

5. CONCLUSIONS $\ldots \ldots \ldots \ldots \ldots \ldots \ldots \ldots \ldots \ldots \ldots \ldots \ldots \ldots \ldots \ldots \ldots$

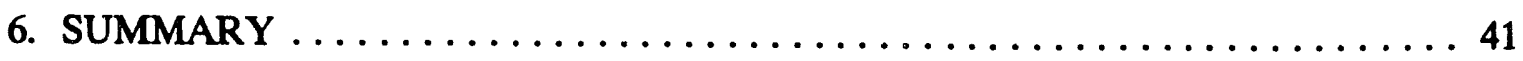

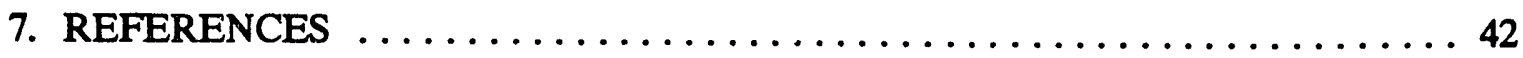

Appendix A. PHYSICAL-CHEMICAL MEASUREMENTS AND CONTAMINANT ANALYTICAL RESULTS FOR 24-h COMPOSITE SAMPLES

FROM THE K-1513 AND WOC STATIONS ............. A-1

Appendix B. ROUTINE WATER QUALITY AND CONTAMINANT RESULTS FROM THE WOD MONITORING STATION ........... B-1

Appendix C. CONTINUOUS WATER QUALITY MONITORING RESULTS ... C-1

Appendix D. WATER GRAB SAMPLE ANALYTICAL RESULTS FOR MONITORING OF SPECIFIC CONSTRUCTION ACTIVITIES . . . D D-1

Appendix E. QUALITY ASSURANCE/QUALITY CONTROL DATA . . . . . E E-1 


\section{FIGURES}

1 Site map including location of sediment-retention structure and monitoring

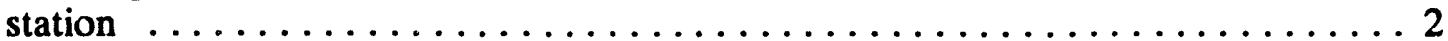

2 Continuous plot of hourly average water discharge from the White Oak Lake Dam, June 1991 through July $1992 \ldots \ldots \ldots \ldots \ldots$

3 Plots of 24-h composite samples taken at the mouth of White Oak Creek for (a) total ${ }^{137} \mathrm{Cs}(\mathrm{pCi} / \mathrm{L})$, (b) particle-associated ${ }^{137} \mathrm{Cs}(\mathrm{pCi} / \mathrm{g})$,

(c) $\mathrm{pH},(\mathrm{d})$ total suspended solids (mg/L), and (e) turbidity (NTU)

4 Plots of 24-h composite samples taken at the K-1513 water intake structure for (a) total ${ }^{137} \mathrm{Cs}(\mathrm{pCi} / \mathrm{L})$, (b) particle-associated ${ }^{137} \mathrm{Cs}(\mathrm{pCi} / \mathrm{g})$,

(c) $\mathrm{pH},(\mathrm{d})$ total suspended solids $(\mathrm{mg} / \mathrm{L})$, and (e) turbidity (NTU) $\ldots \ldots \ldots \ldots 18$

5 Continuous plots of (a) temperature, (b) $\mathrm{pH}$, (c) dissolved oxygen, and

(d) stage height for the water quality monitoring station at the mouth of White Oak Creek ........................ 25

6 Continuous plot of $\mathrm{pH}$ values for grout released to surface waters $\ldots \ldots \ldots 31$ 


\section{TABLES}

1 Chronology of construction activities monitored in association with the WOCE time-critical CERCLA removal action $\ldots \ldots \ldots \ldots \ldots \ldots \ldots$

2 Requested analyses and procedures for water quality monitoring in association with the WOCE time-critical CERCLA removal action .......8 8

3 Water quality sampling results from 24-h composite sampler operations at $\mathrm{K}-1513$ and the White Oak Creek mouth stations .............. 23

4 Water quality monitoring results for the White Oak Creek mouth

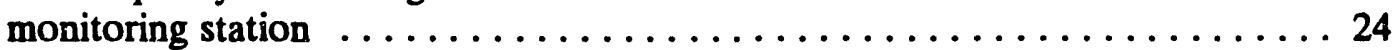

5 Summary of parameters, range, and number of observations for samples collected during sediment-retention structure construction at WOCK $0.0 \ldots 30$

6 Summary of parameters, range, and number of observations for samples collected during sediment dredging activities associated with construction of the sediment-retention structure at WOCK $0.0 \ldots \ldots \ldots \ldots 32$

7 List of parameters, range, and number of observations for samples collected during rock anchor drilling activities associated with construction of the sediment-retention structure at WOCK $0.0 \ldots \ldots \ldots \ldots \ldots$

A.1 Total ${ }^{137} \mathrm{Cs}$ results from $24-\mathrm{h}$ composite samples $\ldots \ldots \ldots \ldots \ldots \ldots \ldots$ A-3

A.2 Water chemistry results from 24 h composite samples $\ldots \ldots \ldots \ldots \ldots \ldots$ A-10

A.3 Composite sampler particle-associated ${ }^{137} \mathrm{Cs}$ results $\ldots \ldots \ldots \ldots \ldots \ldots$ A-22

B.1 Radiological data for White Oak Dam outflows, June 1991 through June $1992 \ldots \ldots \ldots \ldots \ldots \ldots \ldots \ldots \ldots \ldots \ldots \ldots \ldots \ldots$. . . . . . . . . . . . .

B.2 White Oak Dam physical-chemical parameters, June 1991 through July 1992

C.1 Water quality monitoring results for the 1 hite Oak Creek mouth station (CRM 20.8)

D.1 Water grab sample analytical results for monitoring of specific construction activities

E.1 Project quality assurance/quality control data for 24 -h composite samples ... E-3

E.2 Project quality assurance/quality control data for surface water grab samples 


\section{ABBREVIATIONS}

$\begin{array}{ll}\text { ACD } & \text { Analytical Chemistry Division } \\ \text { CERCLA } & \text { Comprehensive Environmental Response, Compensation and Liability Act } \\ \text { CRM } & \text { Clinch River mile } \\ \text { CRRI } & \text { Clinch River Remedial Investigation } \\ \text { EPA } & \text { U. S. Environmental Protection Agency } \\ \text { ER } & \text { Environmental Restoration } \\ \text { ESD } & \text { Environmental Sciences Division } \\ \text { LLRA } & \text { low-level radiological analysis } \\ \text { ORNL } & \text { Oak Ridge National Laboratory } \\ \text { QAVC } & \text { quality assurance/quality control } \\ \text { RAL } & \text { radiochemical analysis laboratory } \\ \text { RCRA } & \text { Resource Conservation and Recovery Act } \\ \text { SOP } & \text { standard operating procedure } \\ \text { SOW } & \text { statement of work } \\ \text { TSS } & \text { total suspended solids } \\ \text { TVA } & \text { Tennessee Valley Authority } \\ \text { WAG } & \text { waste area grouping } \\ \text { WOC } & \text { White Oak Creek } \\ \text { WOCE } & \text { White Oak Creek Embayment } \\ \text { WOD } & \text { White Oak Dam } \\ \text { WOCK } & \text { White Oak Creek kilometer } \\ \text { WOL } & \text { White Oak Lake }\end{array}$




\section{ACKNOWLEDGMENTS}

The authors wish to thank Tom Stephens and Susan Madix of the Environmental Sciences Division and Dan Levine and Scott Niemela of the Automated Sciences Group, Inc., Oak Ridge, Tennessee, for leading the teams that collected and processed the field data and samples. Thanks are also due Craig Brandt of the Engineering Physics and Mathematics Division; Antoinette Brenkert, David Cox, Rhonda Epler, and Jayne Haynes of the Environmental Sciences Division; Mark Bevelhimer, Dennis Crumby, and Don Harris of the Automated Sciences Group, Inc., Oak Ridge, Tennessee; and Jackie Grebmeier and Ken Ham of The University of Tennessee Graduate Program in Ecology for their assistance in field sampling. The untiring efforts put forth by Laury Hamilton and her staff of the ORNL Office of Environmental Health Protection, Environmental Surveillance Protection Section in maintaining and collecting samples from the 24-h composite samplers were invaluable to the project. Special thanks are also due Lauren Larsen of the Environmental Sciences Division and Marion Ferguson, Norman Teasley, and Jeff Wade of the ORNL Analytical Chemistry Division for their cooperation in the high-speed and high-quality analyses of all samples collected.

Data provided by P. Y. Goldberg, M. C. Salmons, and M. M. Stevens of the ORNL Office of Environmental Health Protection, and S. M. Gregory of the Environmental Sciences Division from monitoring of the White Oak Dam were invaluable to this report.

The reviews of this document by H. L. Boston, G. F. Cada, R. B. Cook, and R. R. Turner of the Environmental Sciences Division and B. L. Kimmel of the ORNL Environmental Restoration Division added significantly to the quality of this document and were greatly appreciated. 


\section{EXECUTIVE SUMMARY}

Water quality monitoring activities that focused on the detection of resuspended sediments in the Clinch River were conducted in conjunction with the White Oak Creek Embayment (WOCE) time-critical Comprehensive Environmental Response, Compensation, and Liability Act (CERCLA) removal action to construct a sediment-retention structure at the mouth of White Oak Creek (WOC). Samples were collected by use of a 24-h composite sampler and through real-time water grab sampling of sediment plumes generated by the construction activities. Sampling stations were established both at the WOC mouth, immediately adjacent to the construction site, and at K-1513, the Oak Ridge K-25 Site drinking water intake approximately $9.6 \mathrm{~km}$ downstream in the Clinch River.

Surface water radiological data collected prior to coffer cell closure were used to gauge the effectiveness of the coffer-cell structure at the WOC mouth. These data showed low but measurable quantities of ${ }^{137} \mathrm{Cs}$ leaving the embayment under normal flow conditions.

At both the WOC mouth and $\mathrm{K}-1513$ stations, ${ }^{137} \mathrm{Cs}$ levels were low during late summer and early fall, immediately following closure of the coffer-cell structure. At both stations, ${ }^{137} \mathrm{Cs}$ levels rose with increasing late fall rainstorms, runoff, and increasing TSS levels, a function of seasonal changes in water discharge from the WOC watershed.

Increases in stream $\mathrm{pH}$ caused by the release of cement during jet grout activities were observed at the WOC mouth. Cesium-137 also was observed coincident with jet grouting, though observed levels were comparable with discharges from White Oak Lake (WOL), and the observations may be attributed routine operations at White Oak Dam (WOD). Review of data from 24-h composite samples collected during concrete cap construction do not indicate increased ${ }^{137} \mathrm{Cs}$ or stream $\mathrm{pH}$ levels associated with these activities. No increases in ${ }^{137} \mathrm{Cs}$ or $\mathrm{pH}$ were observed at the $\mathrm{K}-1513$ station during jet grouting.

For both 24-h composite samples (WOC and K-1513) and surface water grab samples ${ }^{137} \mathrm{Cs}$ activity increased in conjunction with sedimerit dredging and rock anchor drilling. At the WOC station, ${ }^{137} \mathrm{Cs}$ values for dredging at WOC were elevated by one to two orders of magnitude relative to nonconstruction reference levels observed from October through February. Values for $\mathrm{K}-1513$ were also elevated for the time frame, though they were one to two orders of magnitude lower than those upstream.

Levels of particle-associated ${ }^{137} \mathrm{Cs}$ at both monitoring stations dropped below detection limits following completion of the sediment-retention structure. However, levels of ${ }^{137} \mathrm{Cs}$ are expected to be comparable to those observed following the establishment of surface-sediment control and nonconstruction monitoring. Cesium-137 releases to the Clinch River from WOD through WOCE appear to be related to sediment transport from WOL during high discharge events. 


\section{INTRODUCTION}

The purpose of the White Oak Creek Embayment (WOCE) time-critical Comprehensive Environmental Response, Compensation, and Liability Act (CERCLA) removal action was to construct a coffer-cell sediment-retention structure at the mouth of WOCE. The sedimentretention structure was designed to contain contaminated sediments in lower WOCE and prevent their transport into the Clinch River for a period consistent with the eventual remeciiation of environmental contamination of the White Oak Creek (WOC) drainage. Other functions of the sediment-retention structure are discussed elsewhere (Kimmel and Ford 1991, Leslie and Kimmel 1992, Blaylock et al. 1993). This monitoring effort was focused on (1) the detection of contaminated WOCE sediments that, if disturbed, could be transported into the Clinch River during construction activities and (2) postconstruction monitoring to assess the effectiveness of the sediment-retention structure. The purpose of this report is to present and discuss the results of water quality monitoring activities from September 1991 through July 1992.

\subsection{SITE BACKGROUND}

WOC is the primary surface-water drainage for the Oak Ridge National Laboratory (ORNL) area (Fig. 1). The WOC drainage is described elsewhere (Blaylock et al. 1993). White Oak Lake (WOL) is a small impoundment of WOC that has served as a settling basin for low-level radioactive effluents from ORNL since 1943. The WOL dam, located approximately $1 \mathrm{~km}$ above the confluence of WOC and the Clinch River, is the last controlled ORNL discharge point on WOC (Blaylock et al. 1993). The WOCE extends downstream of the WOL dam to the (CRM) 20.8 (Fig. 1).

Water flow conditiciss at the mouth of the embayment are regulated by operation of hydroelectric generators at the Melton Hill dam, located on the Clinch River $4.2 \mathrm{~km}$ upstream from the WOC mouth. Tivis hydroelectric capacity is used to meet peak demands in the Tennessee Valley Authority's (TVA's) power production grid, usually generating electricity twice daily. The release of r vater associated with initiation of hydroelectric power generation causes water to flow upstream into WOCE. This surge is held in the embayment by the increased water level in the river resulting from hydroelectric generation. Upon completion of hydropower operation, the water level in the river decreases, and the water Jow in WOC reverses again, moving water from the WOCE into the Clinch River. Prior to construction of the sediment-retention structure, water depth changed by $2 \mathrm{ft}$ or more throughout the embayment in as little as $5 \mathrm{~min}$ following hydropower start-up or shutdown (Blaylock et al. 1993). The hydrodynamics of the WOCE are described elsewhere (Kimmel and Ford 1991, Blaylock et al. 1993). 


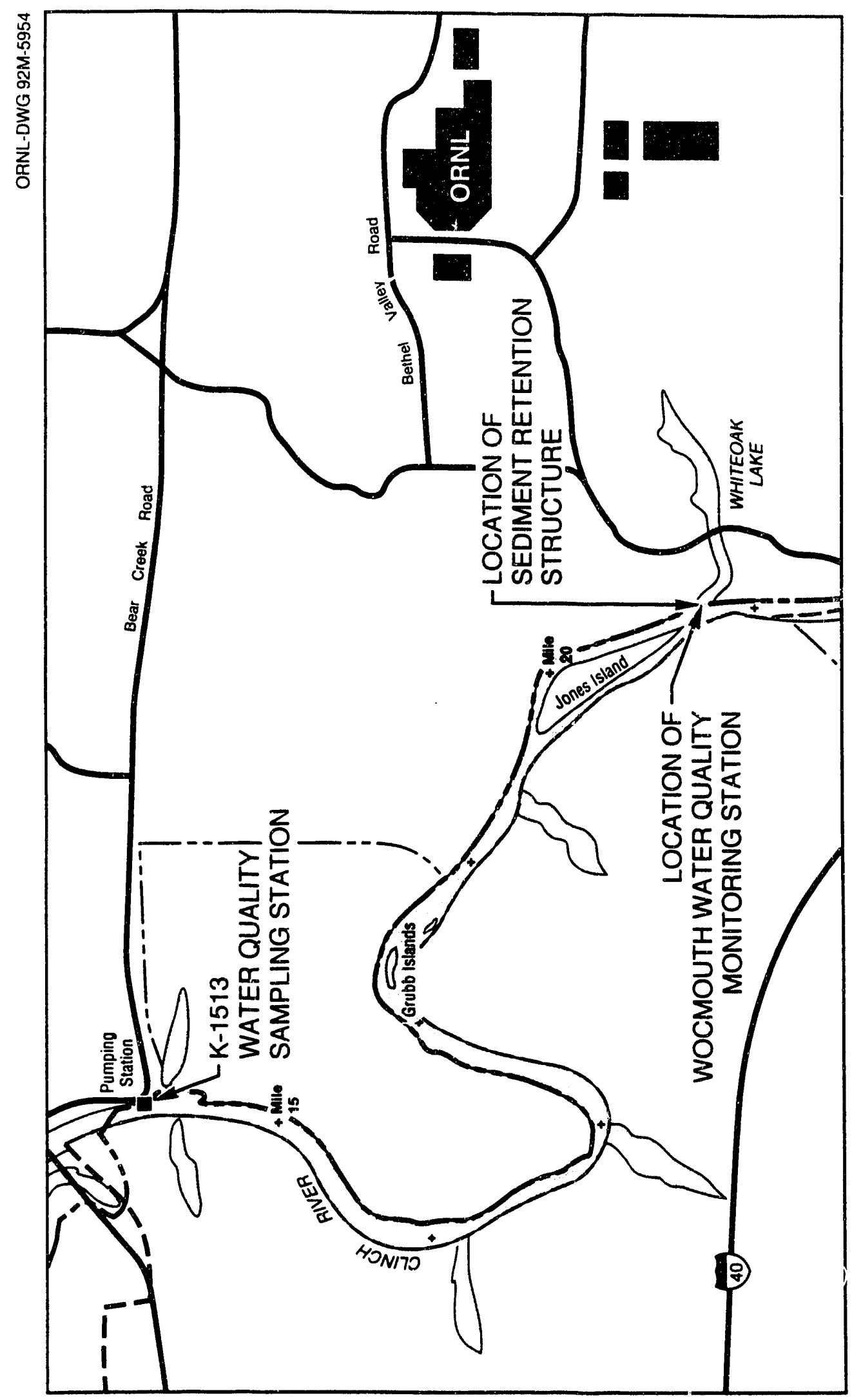

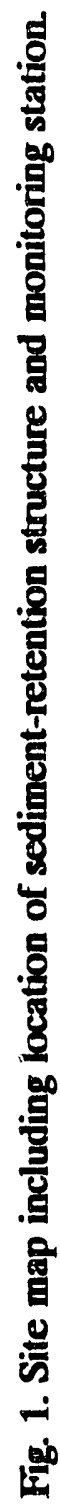




\subsection{WOCE SITE CHARACTERIZATION}

\subsubsection{Phase I Sediment Sampling}

Sediment grab and core samples were obtained from the lower portion of WOCE in summer 1990 as part of Phase 1 of the Clinch River Remedial Investigation (CRRI). Analysis of these samples revealed the presence of higher than expected levels of ${ }^{137} \mathrm{Cs}$ activity in near-surface (top $10 \mathrm{~cm}$ ) sediments (Blaylock et al. 1993). Cesium-137 (half-life is 30 years) is a radioactive isotope historically produced by research reactor operations at ORNL. Mercury, chromium, lead, and ${ }^{60} \mathrm{Co}$ have also been observed in the WOCE sediment samples. Studies in 1979 and 1984 of sediment contamination indicated that the most highly contaminated sediment strata were located between 25 and $50 \mathrm{~cm}$ below the sediment surface. The occurrence of these strata had been ascribed to peak historical releases from WOL in the mid-to-late 1950s (Blaylock et al. 1993).

\subsubsection{Characterization of WOCE Sediments}

Extensive characterization of the WOCE sediments was conducted from August 1990 to May 1991. For sediments located at or near the mouth of WOC, the characterization results indicated that the ${ }^{137} \mathrm{Cs}$ contamination occurred in the upper $1 \mathrm{~m}$ of sediment in the main stream channel (Blaylock et al. 1993). The underlying soil layers (soils between $1 \mathrm{~m}$ and the bedrock) were found to be uncontaminated (Fig. 2 in Kimmel and Ford 1991).

\subsection{Characterization of WOCE Surface Wator}

Water samples from WOCE were also wited as part of the CRRI Phase 1 sampling and the WOCE characterization effort (Blaylocis et al. 1993). WOCE surface water carries similar contaminants as found in WOL and its tributaries. Nonradiological inorganic and organic contaminants were not detected in the water column (Blaylock et al. 1993). Particleassociated gamma radiological contamination of WOCE surface waters consisted primarily of ${ }^{137} \mathrm{Cs}$. Concentrated ${ }^{137} \mathrm{Cs}$ values associated with particles (500 to $3000 \mathrm{pCi} / \mathrm{g}$ of suspended matter, $50 \mathrm{pCi} / \mathrm{g}$ minimum detectable quantity) and dissolved in the water (1 to $40 \mathrm{pCi} / \mathrm{L}$ of water, $0.5 \mathrm{pCi} / \mathrm{L}$ minimum detectable quantity) declined slightly with downstream distance in the embayment. These particle-associated values were similar to the average values observed for surface sediments in WOCE (Blaylock et al. 1993). However, ${ }^{137} \mathrm{Cs}$ concentrations were highly variable at the WOC mouth, fluctuating greatly due to WOC discharge and extent of dilution from the Clinch River. Concentrations of other radionuclides have also been observed to fluctuate with water flow conditions at the mouth of the embayment. In the Clinch River, radiological contaminants appear to be rapidly diluted by Clinch River flows, and they are normally not detectable a short distance $(1 \mathrm{~km})$ downstream within the Clinch.

\subsection{OBJECTIVF}

The primary objective of the Water Quality Monitoring Project for the WOCE timecritical CERCLA removal action was to assess and document the impact to water quality before, during, and after the construction of the sediment-retention structure. Construction using normal excavation techniques was not possible because of the ${ }^{137} \mathrm{Cs}$ contamination of the sediments. Construction activities were designed specifically to minimize the disturbance, 
resuspension, and off-site transport of contaminated sediments. This primary objective, summarized in the White Oak Creek Embayment Time-Critical CERCLA Removal Action Water Quality Monitoring Plan (Kimmel and Ford 1991), defined the parameters of the data collected in association with construction activitie.

$>$ The characterization efforts conducted in 1990 and 1991 (Sect. 1.2) indicated that the WOCE contamination is primarily a result of ${ }^{137} \mathrm{Cs}$ accumulation in the embayment sediments. Therefore, monitoring activities associated with construction activities initially focused on

1. determination of ${ }^{137} \mathrm{Cs}$ activity in the water as a direct indicator of contaminated sediment disturbance and transport at the construction site;

2. daily composite-sample determination of suspended sediment concentrations and continuous monitoring of turbidity as add:ional indicators of sediment disturbance and transport at the WOC mouth;

3. continuous monitoring of standard water quality variables $(\mathrm{pH}$, specific conductance, water temperature, and dissolved oxygen concentration) at the WOC mouth; and

4. monitoring of ${ }^{137} \mathrm{Cs}$ levels at the K-25 Site water supply intake (K-1513) located at CRM 14.5, 8.8 river $\mathrm{km}$ downstream from the mouth of WOC.

Construction activities that had a higher probability of disturbing contaminated sediment were identified prior to construction. The focus of the monitoring efforts addressed the specific requirements of each phase of construction (Table 1). Other natural sediment-disturbing forces, such as seasonal movement of suspended sediment by storms, were assessed by comparison of continuous monitoring results during construction with (1) monitoring results from periods when no construction occurred (Appendix A) and (2) monitoring data from water releases over WOD, upstream from the site, for the same time frame (Fig. 2 and Appendix B). Section 2 addresses specific sampling and analysis methods. Sections 3 and 4 present results and discuss the impact of each activity relevant to the monitoring project objectives. 
Table 1. Chronology of construction activities monitored in association with the WOCE time-critical CERCLA removal action

\begin{tabular}{|c|c|c|}
\hline Date & Title for activity & Description/discussion \\
\hline $05 / 31 / 91$ & $\begin{array}{l}\text { Monitoring station } \\
\text { established }\end{array}$ & $\begin{array}{l}\text { Establish locations for collection of water samples } \\
\text { and monitoring during later sediment-retention } \\
\text { structure construction. Background water samples } \\
\text { composited and collected daily with water-quality } \\
\text { monitoring data and stage height recorded } \\
\text { continuously from this date throughout life of the } \\
\text { project. }\end{array}$ \\
\hline $6-8 / 91$ & $\begin{array}{l}\text { Construction and } \\
\text { baseline monitoring }\end{array}$ & $\begin{array}{l}\text { Site preparatior. and other nonsediment-disturbing } \\
\text { activities. }\end{array}$ \\
\hline $8 / 20-27 / 91$ & $\begin{array}{l}\text { First sediment- } \\
\text { disturbing activities }\end{array}$ & $\begin{array}{l}\text { Placement and driving of sheet pile walls into the } \\
\text { WOC streambed. Water sample collection } \\
\text { conducted to detect resuspension of contam } \\
\text { sediments associated with this construction activity } \\
\text { (moderate to low potential for sediment } \\
\text { disturbance). }\end{array}$ \\
\hline $8,27 / 91$ & Coffer-cell closure & Effective control of WOCE sedi, rents. \\
\hline $9 / 6 / 91$ & $\begin{array}{l}\text { Composite sampler } \\
\text { operation }\end{array}$ & $\begin{array}{l}\text { ORNL-ESP maintenance and composite sample } \\
\text { collection from the WOC mouth and } \mathrm{K}-1513 \text { sites } \\
\text { commences. }\end{array}$ \\
\hline $9 / 10 / 91$ & $\begin{array}{l}\text { Coffer-cell } \\
\text { construction }\end{array}$ & Sheet and H-pile work completed. \\
\hline 9/17/91 & $\begin{array}{l}\text { Composite sampler } \\
\text { operation }\end{array}$ & $\begin{array}{l}\text { WOC monitoring station relocated, and new } \\
\text { monitoring equipment installed. }\end{array}$ \\
\hline $\begin{array}{l}9 / 18- \\
10 / 8 / 91\end{array}$ & Rock armoring & $\begin{array}{l}\text { Water sampling associated with shoreline and } \\
\text { discrete monitoring (high potential for sediment } \\
\text { disturbance). }\end{array}$ \\
\hline $10 / 7 / 91$ & $\begin{array}{l}\text { Composite sampler } \\
\text { operation }\end{array}$ & $\begin{array}{l}\text { Composite sampler operation continued. Water- } \\
\text { quality monitoring discontirued. }\end{array}$ \\
\hline 01/24/92 & $\begin{array}{l}\text { Cornposite sampler } \\
\text { operation }\end{array}$ & $\begin{array}{l}\text { Monitoring station relocated to mid WOCE } \\
\text { mouth. }\end{array}$ \\
\hline $2 / 7-2 / 24 / 92$ & Jet grout & $\begin{array}{l}\text { Initial jet grout test work (moderate to low } \\
\text { sediment disturbance, high pH disturbance). }\end{array}$ \\
\hline $3 / 7-25 / 92$ & Jet grout & $\begin{array}{l}\text { Production with sediment heaving (low sediment } \\
\text { disturbance, high } \mathrm{pH} \text { disturbance potential). }\end{array}$ \\
\hline $\begin{array}{l}3 / 26 \& \\
4 / 1 / 92\end{array}$ & Dredging & $\begin{array}{l}\text { Removal of excess sediment from center cell of } \\
\text { dam (high sediment disturbance). }\end{array}$ \\
\hline
\end{tabular}


Table 1 (continued)

\begin{tabular}{l|l|l}
\hline Date & Title for activity & Description/discussion \\
\hline $05 / 31 / 91$ & $\begin{array}{l}\text { Monitoring station } \\
\text { established }\end{array}$ & $\begin{array}{l}\text { Establish locations for collcction of water samples } \\
\text { and monitoring during later sediment-retention } \\
\text { structure construction. Background water samples } \\
\text { composited and collected daily with water-quality } \\
\text { monitoring data and stage height recorded } \\
\text { continuously from this date throughout life of the } \\
\text { project. }\end{array}$ \\
\hline $3 / 27 \& 4 / 5$ & Concrete cap & $\begin{array}{l}\text { Installation of concrete cap (low sediment } \\
\text { disturbance, high pH disturtance). }\end{array}$ \\
\hline $4 / 4-4 / 10 / 92$ & $\begin{array}{l}\text { Rock anchor } \\
\text { drilling }\end{array}$ & $\begin{array}{l}\text { Installation of rock anchors (very high sediment } \\
\text { disturbance). }\end{array}$ \\
\hline $4 / 14 / 92$ & $\begin{array}{l}\text { Composite sampler } \\
\text { operation }\end{array}$ & $\begin{array}{l}\text { Sediment-retention structure complete. Water } \\
\text { sample collection and water-quality sampling } \\
\text { reduced to least weekly. Water-quality monitoring } \\
\text { discontinued. }\end{array}$ \\
\hline $7 / 29 / 92$ & $\begin{array}{l}\text { Composite sampler } \\
\text { opera' ion }\end{array}$ & $\begin{array}{l}\text { Postconstruction monitoring complete. No further } \\
\text { samples collected for project. }\end{array}$ \\
\hline
\end{tabular}




\section{MATERIALS AND METHODS: SAMPLE COLLECTION, ANALYSIS, AND REPORTING}

The methods for accomplishing the monitoring objectives listed above were modified for each sediment-disturbing construction activity. The schematic for continuous monitoring was established prior to initiation of construction. Guidance for real-time monitoring of specific activities was provided by internally distributed statements of work (Ford 1993a), which are summarized below.

Water samples were collected in support of project objectives. Monitoring parameters for the WOC mouth site are listed in Sects. 2.2 and 2.3. Specific details regarding the continuous monitoring stations, data and sample retrieval and equipment maintenance and calibration are found in Sect 4.1 of the Water Quality Monitoring Plan (Kimmel and Ford 1991).

The sampling strategy was modified to address the conditions of specific construction activities. Quality Assurance/Quality Control (QA/QC) requirements for field sampling are found in Sects. 4.4.3 and 5.4 of the Water Quality Monitorir - Plan (Kimmel and Ford 1991). Field and analytical $r$-ta quality object es were focused on the monitoring requirements for specific construction activities, as described in the activity-specific statements of work (SOWs) (Ford 1993a).

Weather conditions, equipment function, and a description of monitoring activities, as well as certain field measurements, were recorded in field notebooks or on specially designed data forms. All entries into field notebooks followed the standard operating procedure (SOP) "Data Entry and Recording of Technical Information" (SOP-1, addendum to ES/ER/INT-72). Specific precautions regarding the detection of any material resuspended by construction activities and potentialiy contaminated with ${ }^{137} \mathrm{Cs}$ and other particle-reactive contaminants were included in the activity-specific SOWs (Ford 1993a).

\subsection{MONITORING STATIONS, STRATEGY, AND METHODS}

Composite water sample collection stations were established at the WOC mouth (CRM 20.8) and at the K-1513 water intake structure (CRM 14.5). The monitoring station at the WOC mouth consisted of two PVC pipes in the embayment mouth at mid channel. These pipes served as a stilling well, a composite-sample collection point, and a platform for in situ monitoring equipment. The data logging equipment, connected to monitoring instrumentation submersed on these platforms, was housed in an onshore building.

Composite water samples were collected from the two stations (Table 2) and submitted for determination of particle-associated and total ${ }^{137} \mathrm{Cs}$, total suspended solids (TSS), turbidity, and $\mathrm{pH}$ for 24-h composite water samples. Water quality monitoring for temperature $\mathrm{pH}$, dissolved oxygen content, and water stage height was also conducted at the WOC mouth station. Monitoring of $24-\mathrm{h}$ composite sample $\mathrm{pH}$ began during jet grout activities. 
Table 2 Requested analyses and procedures for water-quality monitoring in association with the WOCE time-critical CERCLA removal action.

\begin{tabular}{|c|c|c|}
\hline Analysis & $\begin{array}{l}\text { Container type } \\
\text { (matrix/tield } \\
\text { preservative) }\end{array}$ & $\begin{array}{l}\text { Analytical } \\
\text { procedure }\end{array}$ \\
\hline Total suspended solids & $\begin{array}{l}\text { 1-L HDPE bottle } \\
\text { (residue/chill) }\end{array}$ & EPA $160.2^{b}$ \\
\hline $\mathrm{pH}$ & $\begin{array}{l}\text { 1-L HDPE bottle } \\
\text { (water/chill) }\end{array}$ & EPA $150.1^{b}$ \\
\hline Turbidity & $\begin{array}{l}\text { 1-L HDPE bottle } \\
\text { (water/chill) }\end{array}$ & EPA $180.1^{b}$ \\
\hline Gamma spectroscopy (total, $\mathrm{pCi} / \mathrm{L}$ ) & $\begin{array}{l}\text { 1-L Marinelli beaker } \\
\text { (water/acidified) }\end{array}$ & EPA $901.1^{c}$ \\
\hline $\begin{array}{l}\text { Gamma spectroscopy (particle- } \\
\text { associated, pCi/g) }\end{array}$ & $\begin{array}{l}\text { Cartridge filter } \\
\text { (residue/filter, chill) }\end{array}$ & $\begin{array}{l}\text { EPA 901.1 } \\
\text { Ford 1993a }\end{array}$ \\
\hline
\end{tabular}

${ }^{2} \mathrm{HDPE}=$ high-density polyethylene.

'EPA 1980.

'EPA 1979.

Note: Sample analysis per site, per date to be performed by ORNL ACD following sample delivery.

The general strategy for all sampling fi cused on monitoring resuspended materials rather than collecting a given number of samp a sediment or cement-grout plume, presuming that all samples were collected from a visible plume with a single point source and constant rate of generation. Sample-collection conditions, influenced by river flows, determined qualitatively, and visual extent of the plume on the surface, were as follows:

1. Under high flow conditions (hydroelectric generation at Melton Hill dam), water quality data and sample collection occurred first within a few meters of the plume geseration point, second at approximately $10 \mathrm{~m}$ downstream, and finally at approximately $100 \mathrm{~m}$ downstream.

2. Under baseline flow conditions (no generation from Melton Hill dam), a series of samples were collected over a given period from a stationary point representative of the plume.

For either set of conditions, the optimum rate of sample collection was never more than 3 samples per hour. Actual timing, frequency, and distance of sample collection from construction activities were determined by the sampling team leader. The specific construction activities monitored are listed in Sect. 1.4 and Table 1. Due to the time-critical nature of these data, all radiological sample results during construction monitoring were requested with a 24- to 48-h turnaround. Data were returned directly to the project coordinator (Ford 1993a). Results were reviewed by the project coordinator and evaluated relative to construction-specific SOWs regarding monitoring activities (Ford 1993a). 


\section{WATER QUALITY MONITORING VARIABLES}

Water quality variables monitored continuously from the WOCE station with multiparameter monitoring instruments (Hydrolab Corporation, Austin, Texas) included temperature, $\mathrm{pH}$, conductivity, and dissolved oxygen. Monitoring was modified to meet the objectives of particular contaminant release events. In accordance with manufacturer's instructions, Hydrolab instruments were calibrated to traceable standards. Deviations from calibration for particular instruments were recorded on calibration log sheets. Calibration for continuous monitoring devices was conducted biweekly. Hydrolab instruments used for monitoring from a boat were calibrated prior to sampling, and the calibrations were checked upon return to the laboratory.

The initial focus of this monitoring study included continuous nephelometric monitoring of turbidity in situ to detect construction-related events. However, during the early portions of the monitoring task, the limitations of continuous turbidity measurements were identified, including difficulty in calibration, nonavailability of replacement parts for the instrument, and relatively high ambient turbidities at the WOC mouth. Calibration of the in situ nephelometer was not maintained, and nephelometric monitoring data are therefore not reported. Turbidity was determined in the laboratory using a Hach instrument for each sample collected for this project (Sect. 2.3).

Data on water discharged from the WOL dam from June 199i through July 1992 in cubic feet per second (Fig. 2) were obtained from the ORNL Environmental Restoration (ER) Division Waste Area Grouping 2 (WAG 2) project. Monthly and quarterly radiological and water quality data were also obtained from the ORNL Environmental Monitoring and Compliance Group. These data provide information on sources of suspended solids and contaminants that contributed to observed values at the WOC mouth as well as comparable water quality monitoring information for this project.

\section{REQUESTED ANALYSES AND PROCEDURES}

All samples were analyzed as specified in Sect. 4.3 of the Water Quality Management Plan (Kimmel and Ford 1991) (Table 2). Determination of pH was added during jet grouting activities (Communication WOCE1030692A.SAP in Ford 1993a). Samples submitted to the ORNL Analytical Chemistry Division (ACD) Inorganic Analysis Group were analyzed using NPDES procedures 180.1 for turbidity, 160.2 for TSS, and 150.1 for pH (EPA 1979).

Samples submitted to the ORNL-ACD Low-Level Radiological Analysis (LLRA) group were analyzed using Environmental Protection Agency (EPA) method 901.1, gammaspectrometry (EPA 1980). Additional field samples were analyzed for gamma spectrometry by the Environmental Sciences Division (ESD) Radiochemical Analysis Laboratory (RAL). All gamma spectrometry samples were placed on the detectors for 100 -min counts. Results reported in either picocuries or becquerels per unit mass or volume were converted to picocuries for this report $(1 \mathrm{~Bq}=27.03 \mathrm{pCi})$. Preparation and use of appropriate standard geometries for the samples to be counted were performed by the analytical laboratories.

Particle-associated ${ }^{137} \mathrm{Cs}$ results (in picocuries per gram) from gamma-spectrometry of high-capacity cartridge filters may greatly exceed ${ }^{137} \mathrm{Cs}$ results for total ${ }^{137} \mathrm{Cs}$ activity (in 


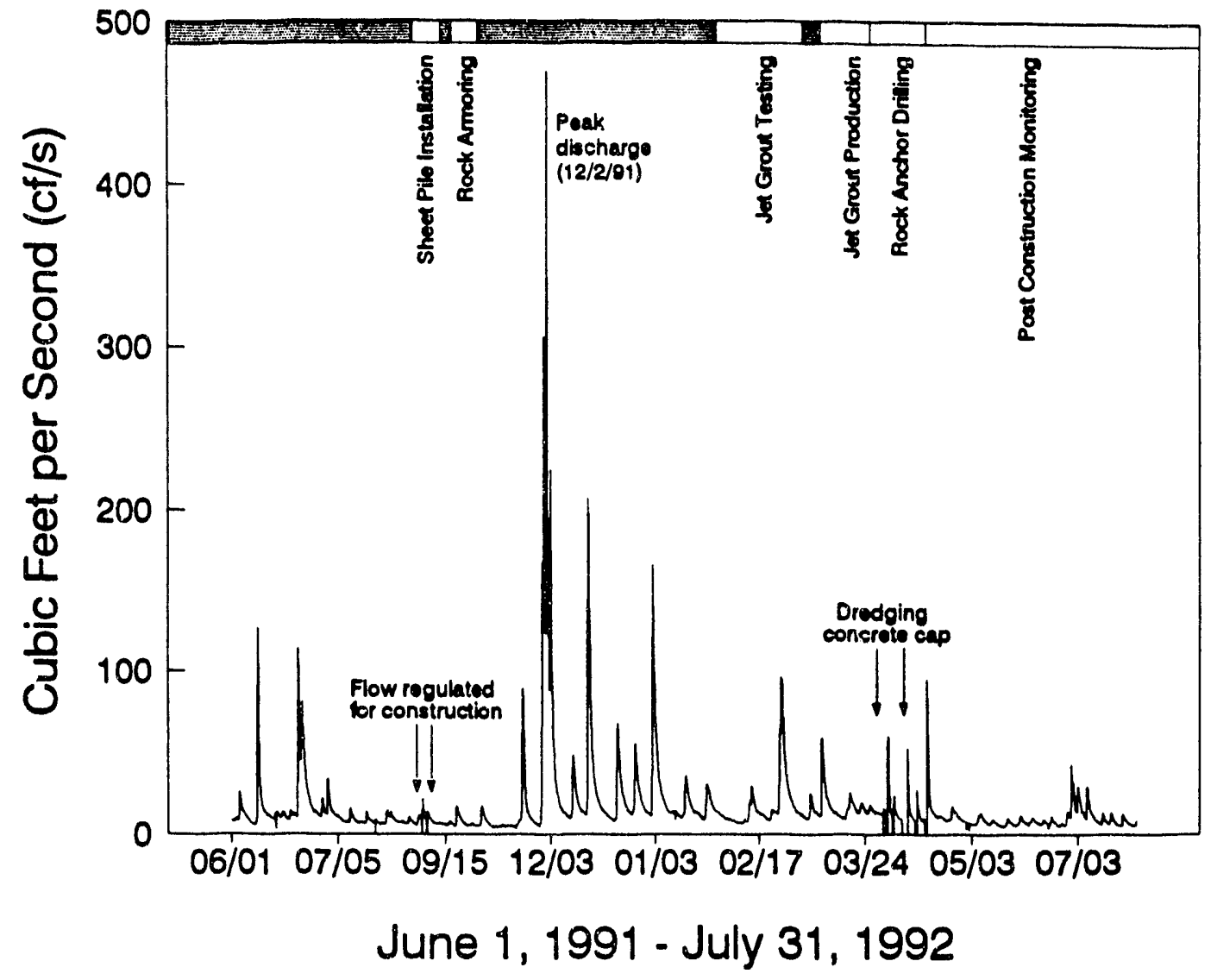

Fig. 2 Continuous plot of hourly average water discharge from the White Oak Lake Dam, June 1991 through July 1992 
picocuries per gram). The use of cartridge filters for surface water samples has provided much improved detection limits, allowing for direct comparison of surface water and surface sediment contaminant concentrations. However, data for water placed in Marinelli beakers of water for gamma spectrometry are the basis for comparison with regulatory limits.

\section{PREANALYTICAL PROCESSING}

Samples collected through use of a time-proportional composite sampler set to collect $0.1 \mathrm{~L}$ of sample every 10 to $30 \mathrm{~min}$, depending upon monitoring requirements, were apportioned in the field into four aliquots, as summarized in Table 2 . The first and second aliquots consisted of duplicate prelabeled 1-L I-Chem bottles submitted for turbidity and TSS analyses. For the third aliquot, $900 \mathrm{~mL}$ of well-mixed sample was placed in a prelabeled 1-L Marinelli beaker and acidified. The fourth aliquot, which consisted of sample material remaining in the sample carboy ${ }_{3}$ was processed through a cartridge filter. The third and fourth aliquots were analyzed $b_{y}$ gamma spectrometry.

\section{CHAIN OF CUSTODY}

Sample custody was maintained cand documented as specified in guidance SOWs (Ford 1993a) using standard CRRI procedures (ES/ER/INT-72) and the Water Quality Management Pian (Kimmel and Ford 1991). Chain-of-custod; definitions and procedures followed those of "Sample Custody Procedures" (Sect. 10.3 in Energy Systems 1990) and Energy Systems ESP-500 (Kimbrough et al. 1990).

\section{¿6 SAMPLE IDENTIFICATION}

The sample identification codes used for this project were specified in weekly communications from the project coordinator to the environmental sampling teams (Ford 1993b). All results reported from the analytical laboratories used this standard sample identification format.

\section{QUALTTY ASSURANCERUALTTY CONTROL}

QA/QC procedures for this project are detailed in Sect. 5.4 of the Water Quality Management Plan (Kimmel and Ford 1991). Laboratory QA/QC piocedures are discussed in Communication WOCEl070891A.SOW (Ford 1993a). Routine field quality control samples, consisting of field duplicates and sampling device rinse water samples, were collected as specified in sample identification communications (Ford 1993b). All QA/QC field data for this project are reported in Appendix E. Records management for this project was specified in Sect. 5.5 of the Water Quality Management Plan (Table 4 in Kimmel and Ford 1991). 


\section{RESULTS}

WOD discharge values in cubic feet per second, for the period June 1991 through July 1992, are presented in Fig. 2. This plot illustrates the highly variable nature of WOD discharges. WOD flows were reduced to nearly zero during critical construction phases, including installation of final sheet pilings, dredging of excess material from inside the structure, and installation of the concrete cap. Typically, Melton Hill Dam discharges dilute those from "NOD by a ratio of 50 to 500 within the Clinch River, depending upon flows. Discharges from WOC may not be fully mixed with the Clinch River and the full dilution not observed for 10 to $15 \mathrm{~km}$ downstream from the WOC mouth (Dennis Borders, ORNL, personal communication).

\subsection{COMPOSITE SAMPLING}

Total and particle-associated ${ }^{137} \mathrm{Cs}$, and TSS for 24-h composite water samples collected from stations located approsimately in the WOC mouch (Fig. 3) and at the K-1513 water intake structure (Fig. 4) are summarized in Table 3. Monitoring results from the analytical chemistry laboratories are presented in Appendix A. Fiesults for turbidity and TSS analyses are seasonally variable, reflecting changes in discharge: from WOC.

Review of QA/QC data (Appendix E) indicated that (1) duplicate samples for both composite and grab samples had standard error values of $10 \%$ or less, (2) rinse blanks for field data throughout this project did not yield detectable valu/ss of residual contamination, and (3) rinse blanks for composite samplers initially were low but showed some elevated levels of contaminants ciuring the spring (Appendix E). The increasing rinse-composite-sampler rinse-blank results may be the result of the inefficient cleaning methods used for composite sampling equipment.

Radiological data collected during the preconstruction period at the WOC mouth show low but measurable concentrations of ${ }^{137} \mathrm{C}$ leaving WOC during normal flow conditions prior to coffer-cell closure. These values are comparable with results from the WOD monitoring station collected over the same time period (Kornegay et al. 1992 and App endix B). These values dropped and remained low during the early fall of 1991 (Fig. 3). Levels of ${ }^{137} \mathrm{Cs}$ and TSS concentrations rose during the late fall but declined during the winter and remained low, normally below the counting error throughout December, January, and part of February (see Figs. 3a, 3b, 3d, and 3e). The peak total ${ }^{137} \mathrm{Cs}$ value for $\mathrm{K}-1513$ was observed in conjunction with a high volume discharge from WOL (Fig. 2), presumably associated with a rain event in the WOC watershed. With this exception, data from the K-1513 station during the fall and winter showed little or no detectable ${ }^{137} \mathrm{Cs}$ concentrations (Figs. 4a, 4b, 4d, and $4 \mathrm{e}$ ).

There were increases in radiological results from 24-h composite samples coincident with jet grouting, concrete cap construction, sediment dredging, and rock anchor drilling at the WOC mouth station (Figs. $3 \mathrm{a}$ and $3 \mathrm{~b}$ ). Dredging and drilling activities also increased the ${ }^{137} \mathrm{Cs}$ levels at the K-1513 station (Figs. $4 \mathrm{a}$ and $4 \mathrm{~b}$ ). Levels of radiological contaminants at both monitoring stations dropped following completion of the sediment-retention structure (Figs. 3a, 3b, 4a, and 4b; Appendix A). 


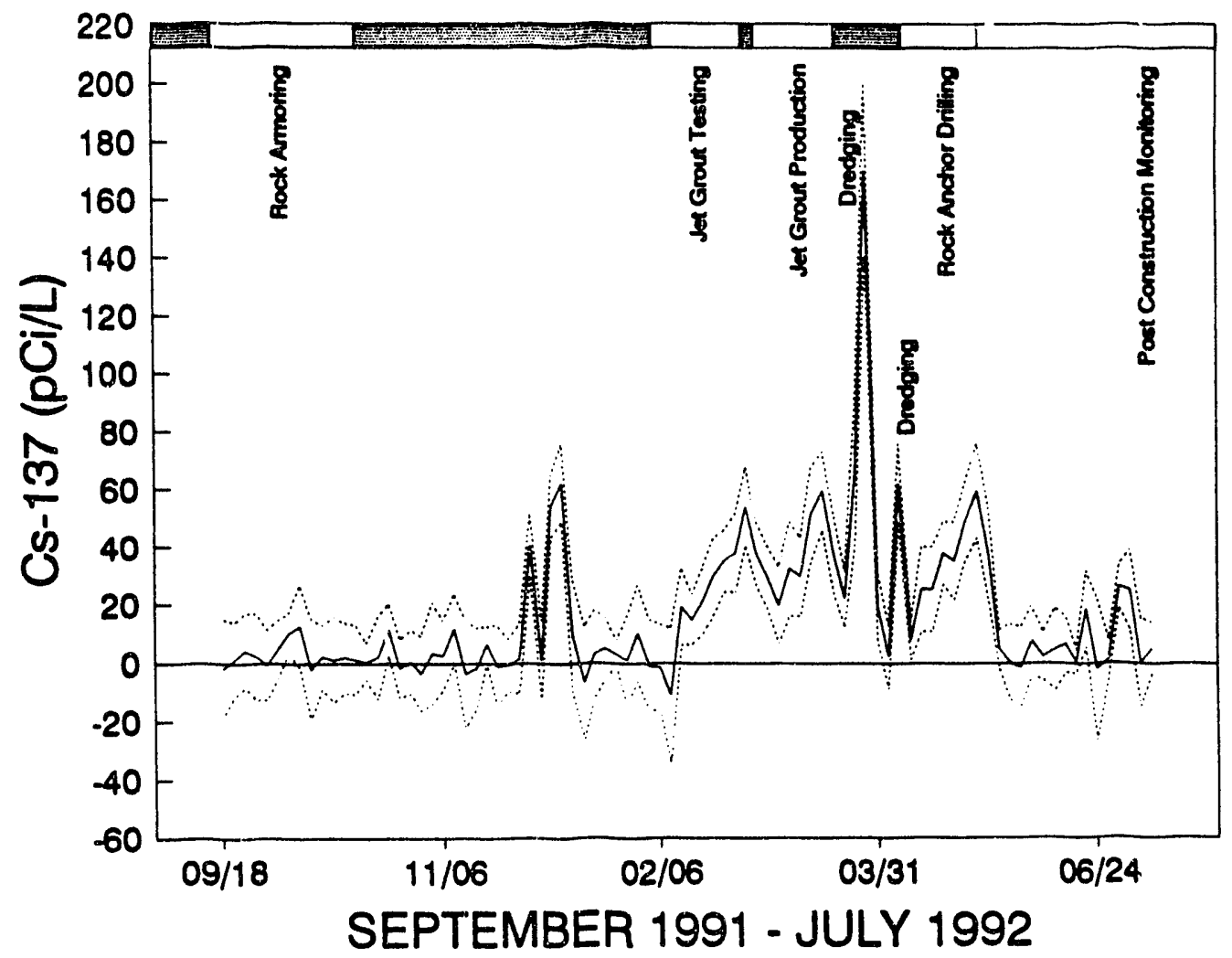
Creek.

Fig. 3a. Plot of total ${ }^{137} \mathrm{Cs}(\mathrm{pCi} / \mathrm{L})$ in 24 -h composite samples at the mouth of White Oak 


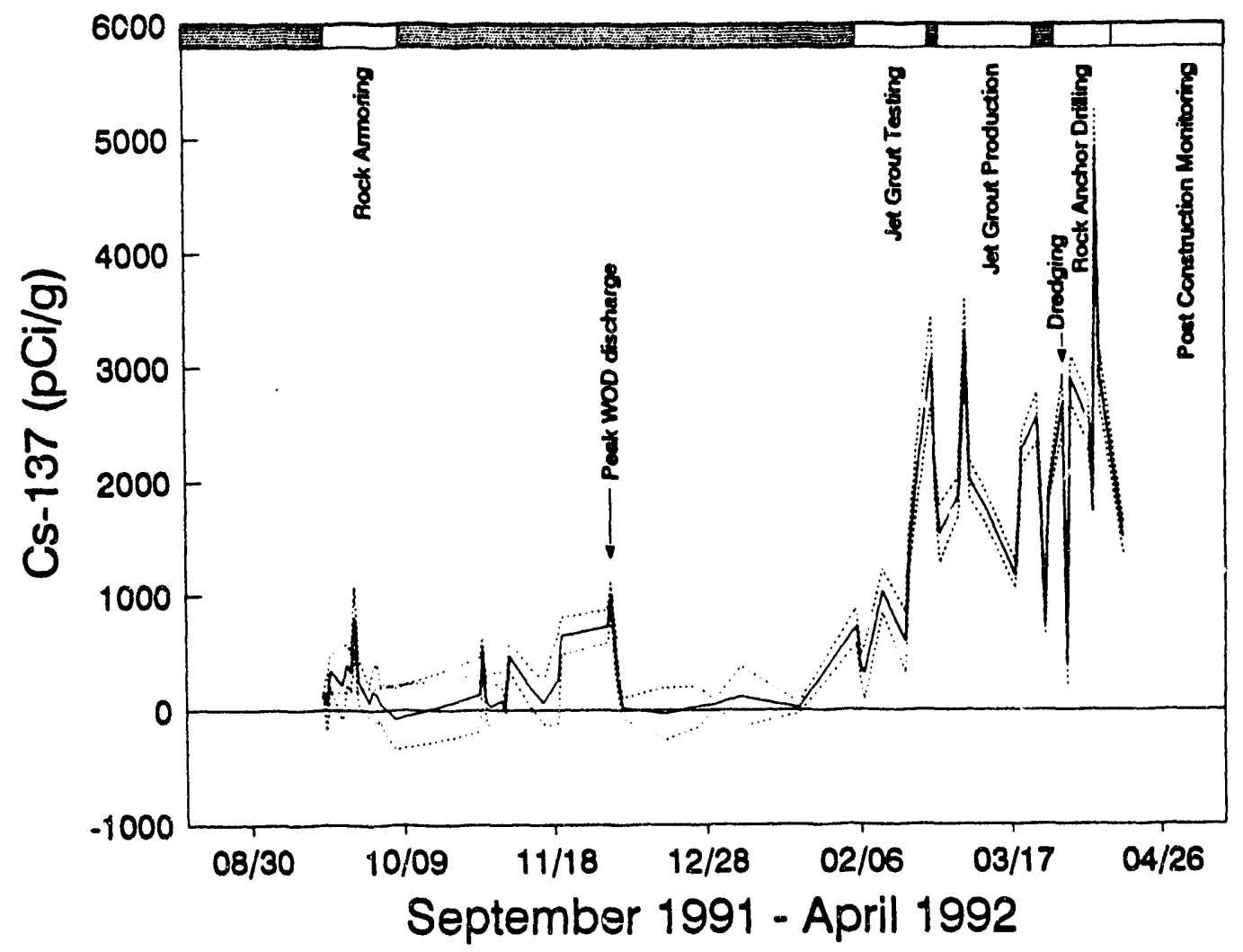

Fig. 3b. Plot of particle-associated ${ }^{137} \mathrm{Cs}(\mathrm{pCi} / \mathrm{g})$ in 24 -h composite samples at the mr uth of White Oak Creek. 


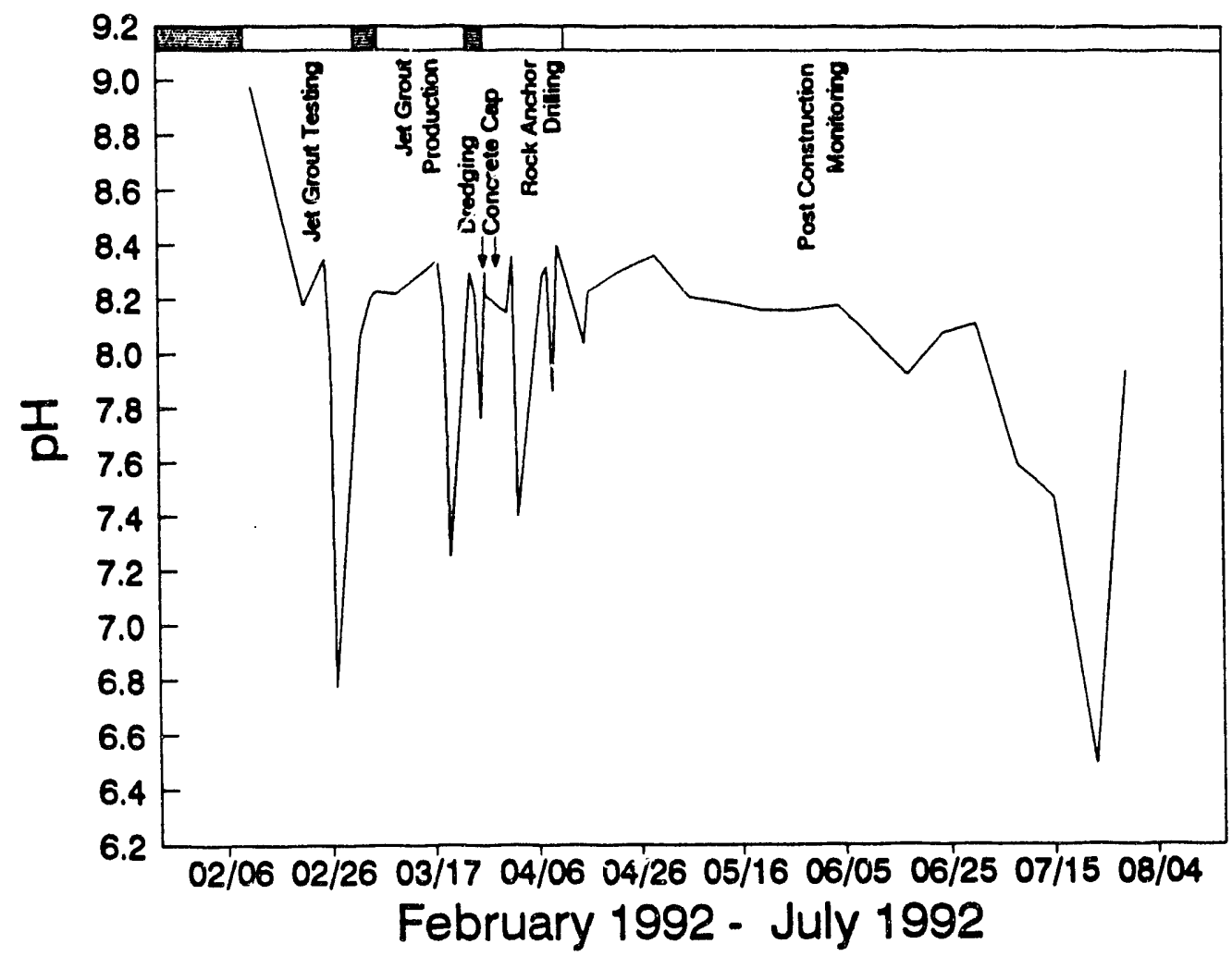

Fig. 3c. Plot of $\mathrm{pH}$ in 24-h composite samples at the mouth of White Oak Creek. 


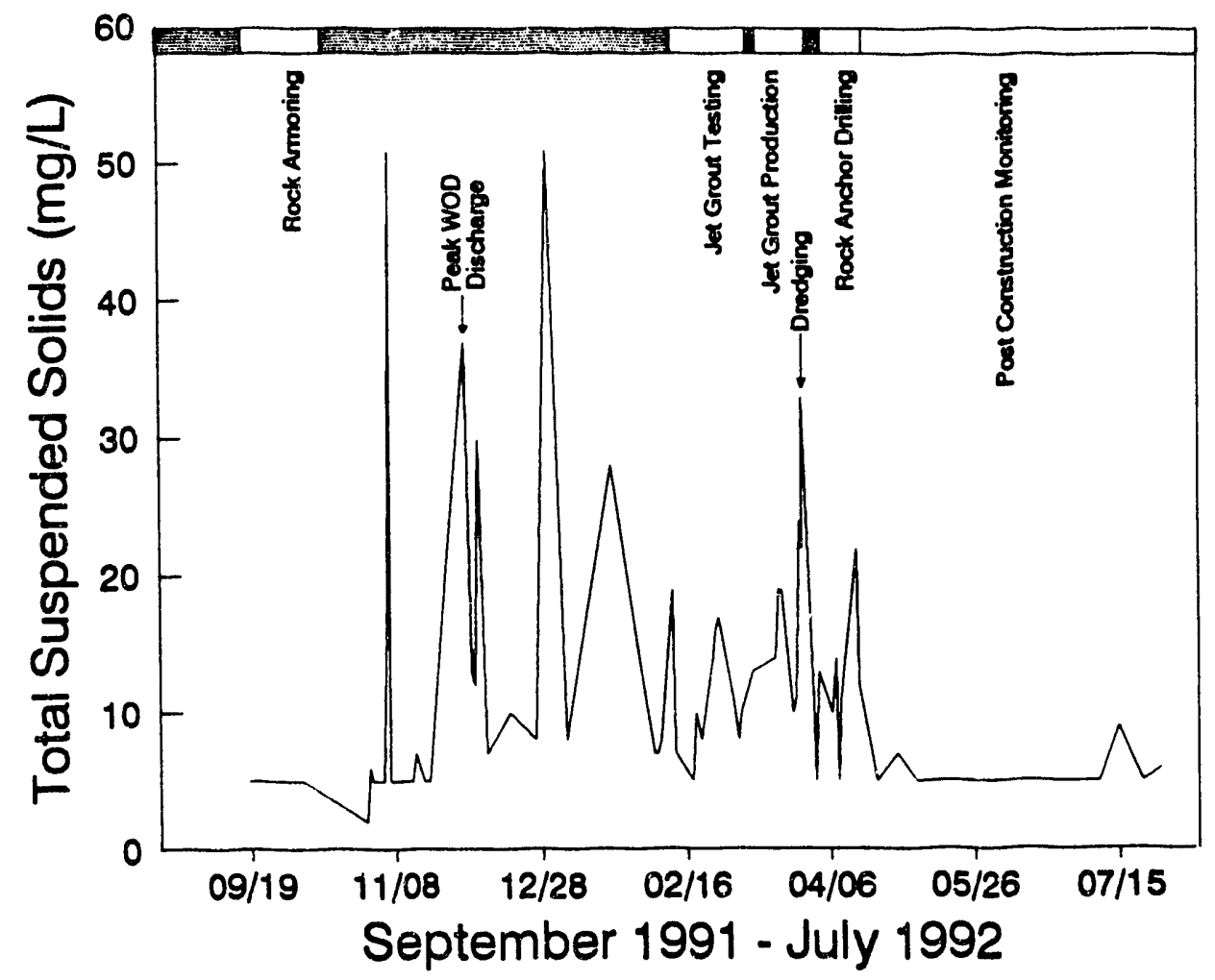

Fig. 3d. Plot of total suspended solids (mg/L) in 24-h composite samples at the mouth of White Oak Creek. 


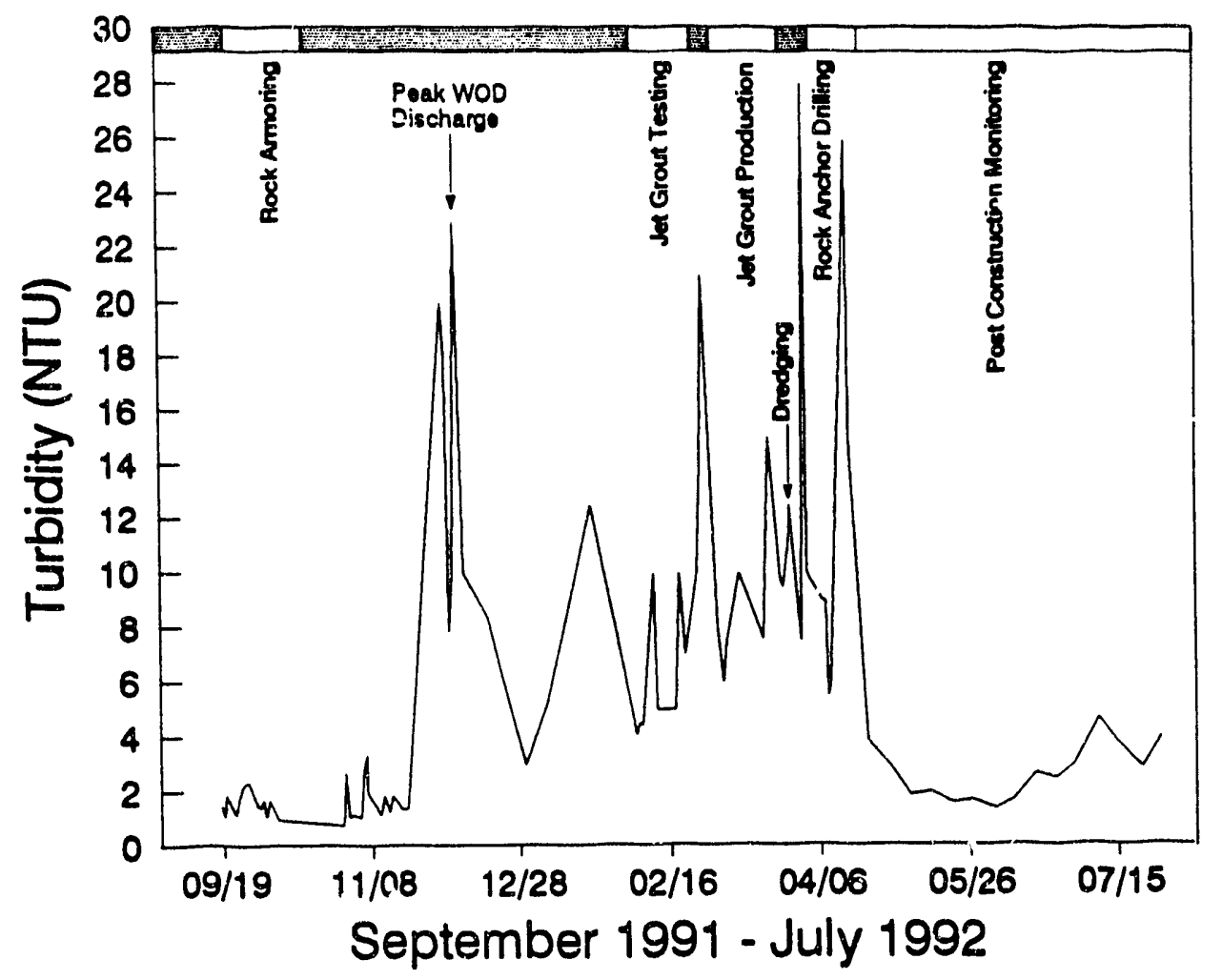
Creek

Fig. 3e. Plot of turbidity (NTU) in 24-h composite samples at the mouth of White Oak 


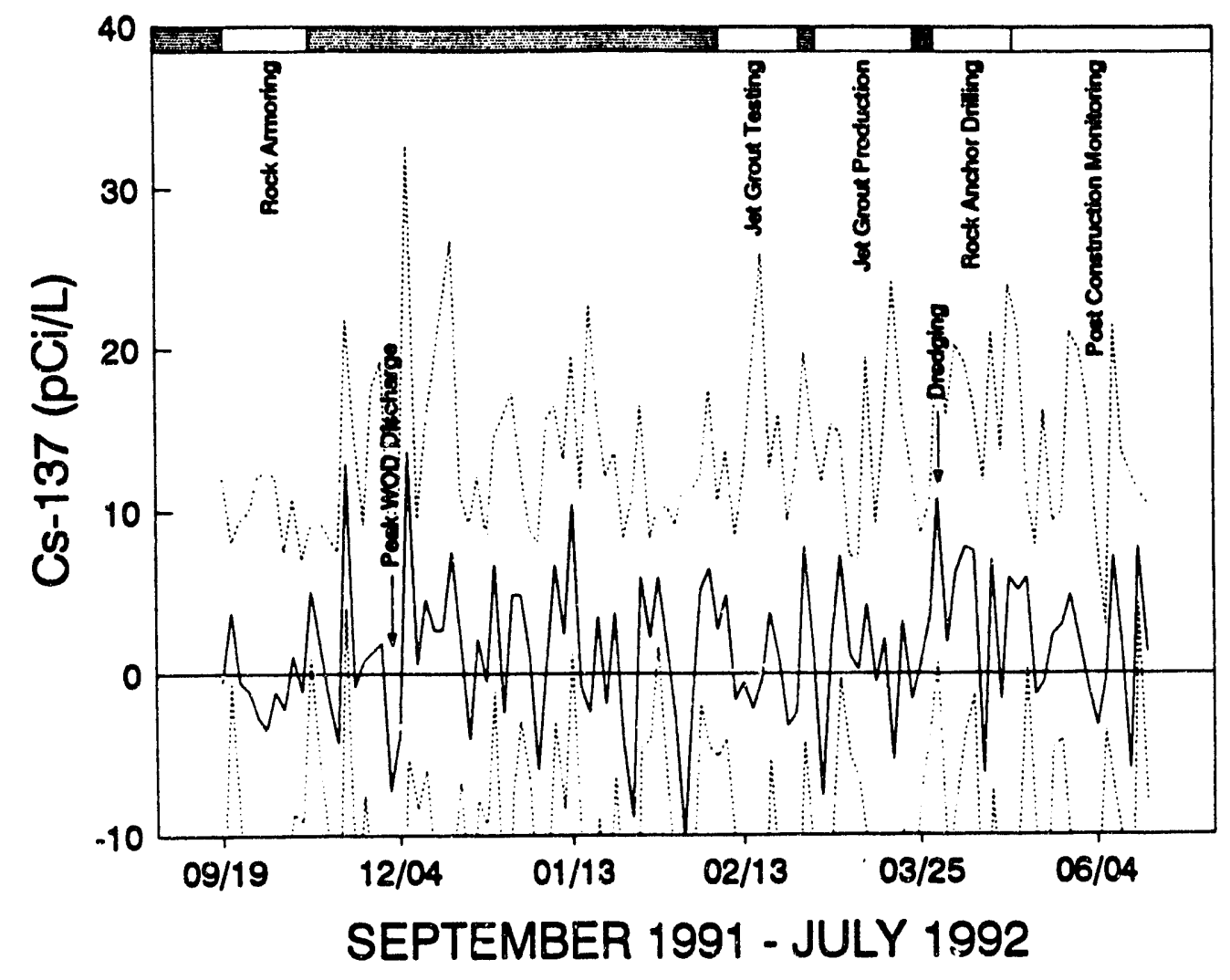

Fig. 4a. Plot of total ${ }^{137} \mathrm{Cs}$ ( $\left.\mathrm{pCi} / \mathrm{L}\right)$ in $24 \mathrm{~h}$ composite samples at the $\mathrm{K}-1513$ water intake structure. 


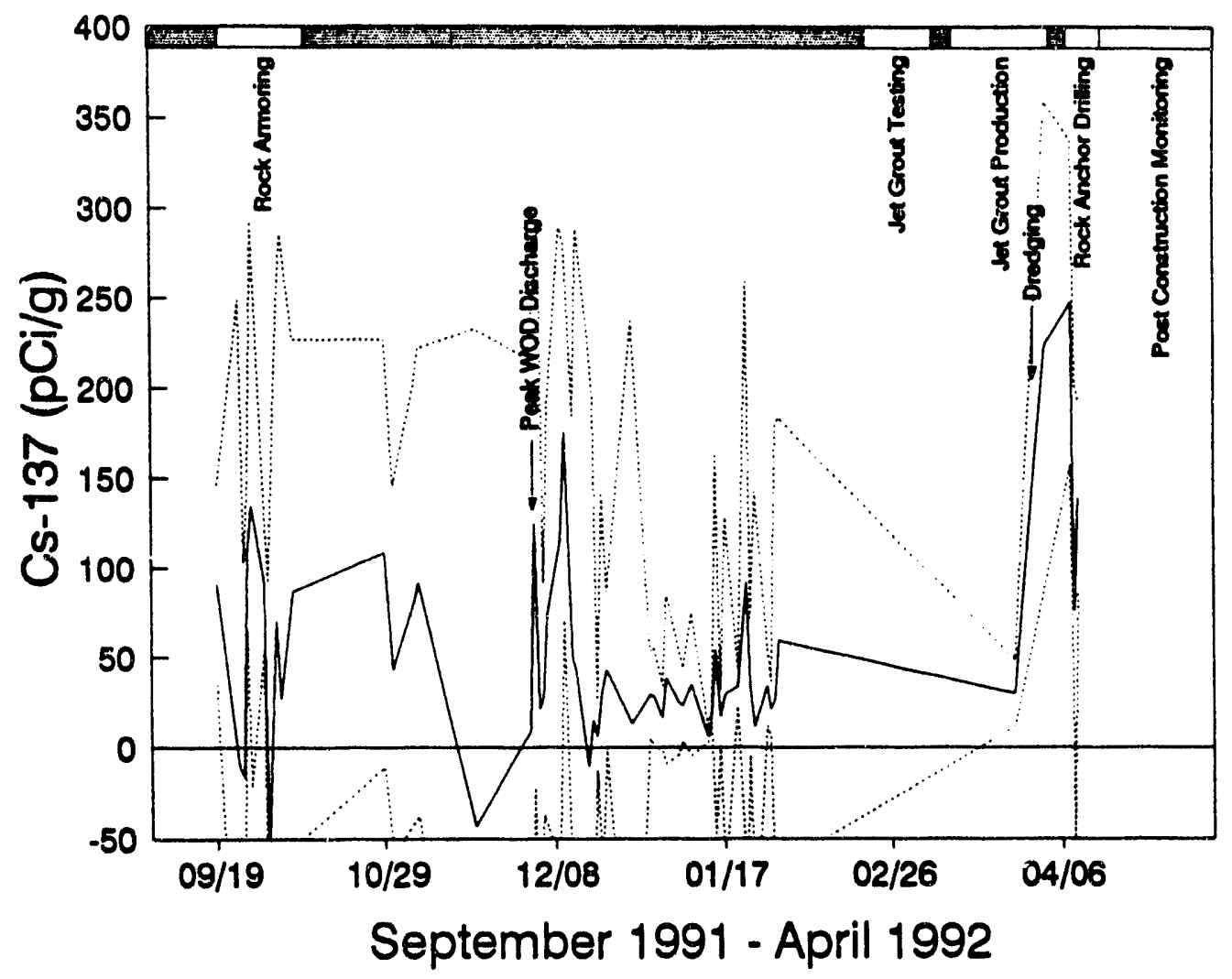

Fig. 4b. Plot of particle-associated ${ }^{137} \mathrm{Cs}$ (pCi/g) in 24h composite samples at the K-1513 water intake structure. 


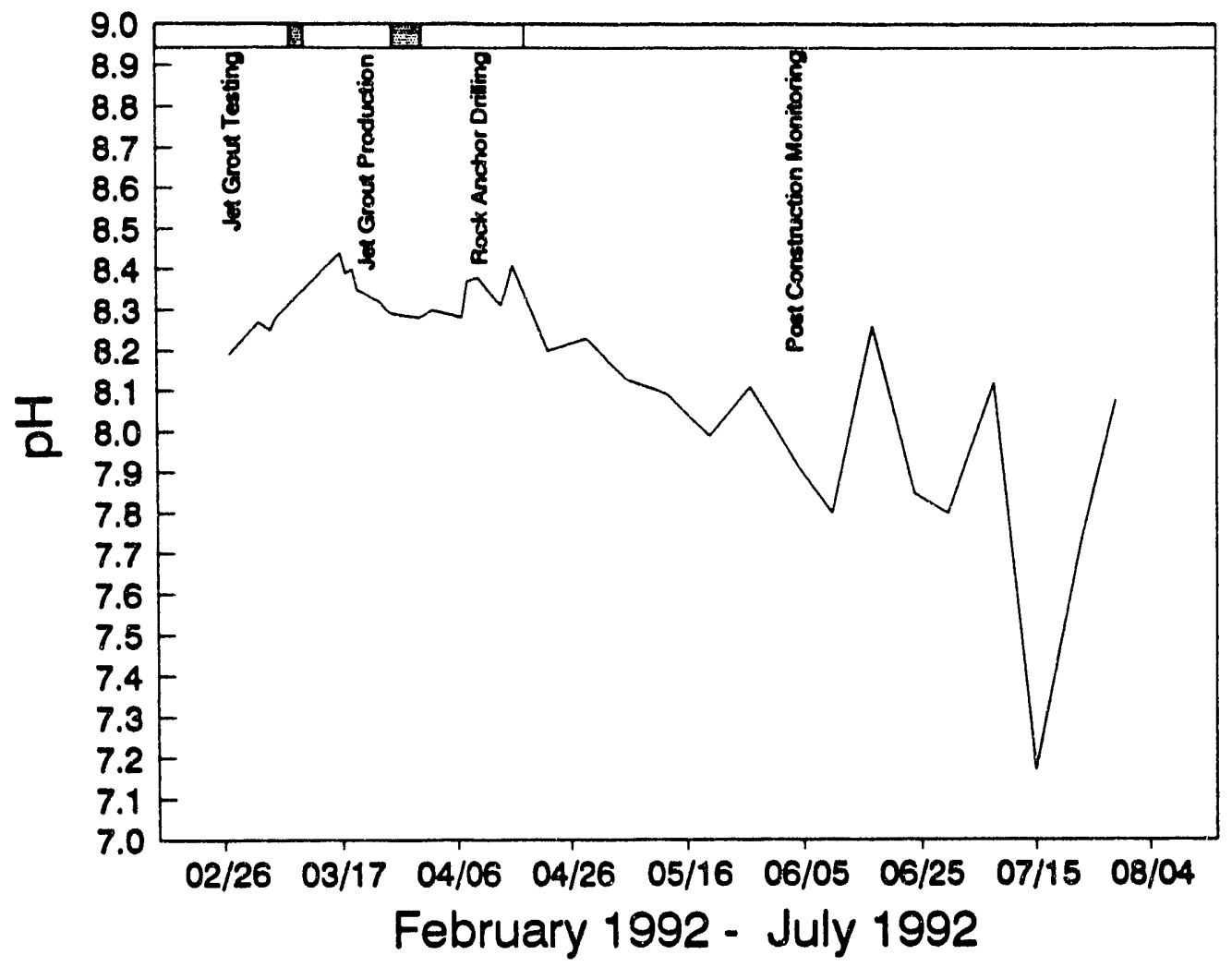

Fig. 4c. Plot of pH in 24-h composite samples at the K-1513 water intake structure. 


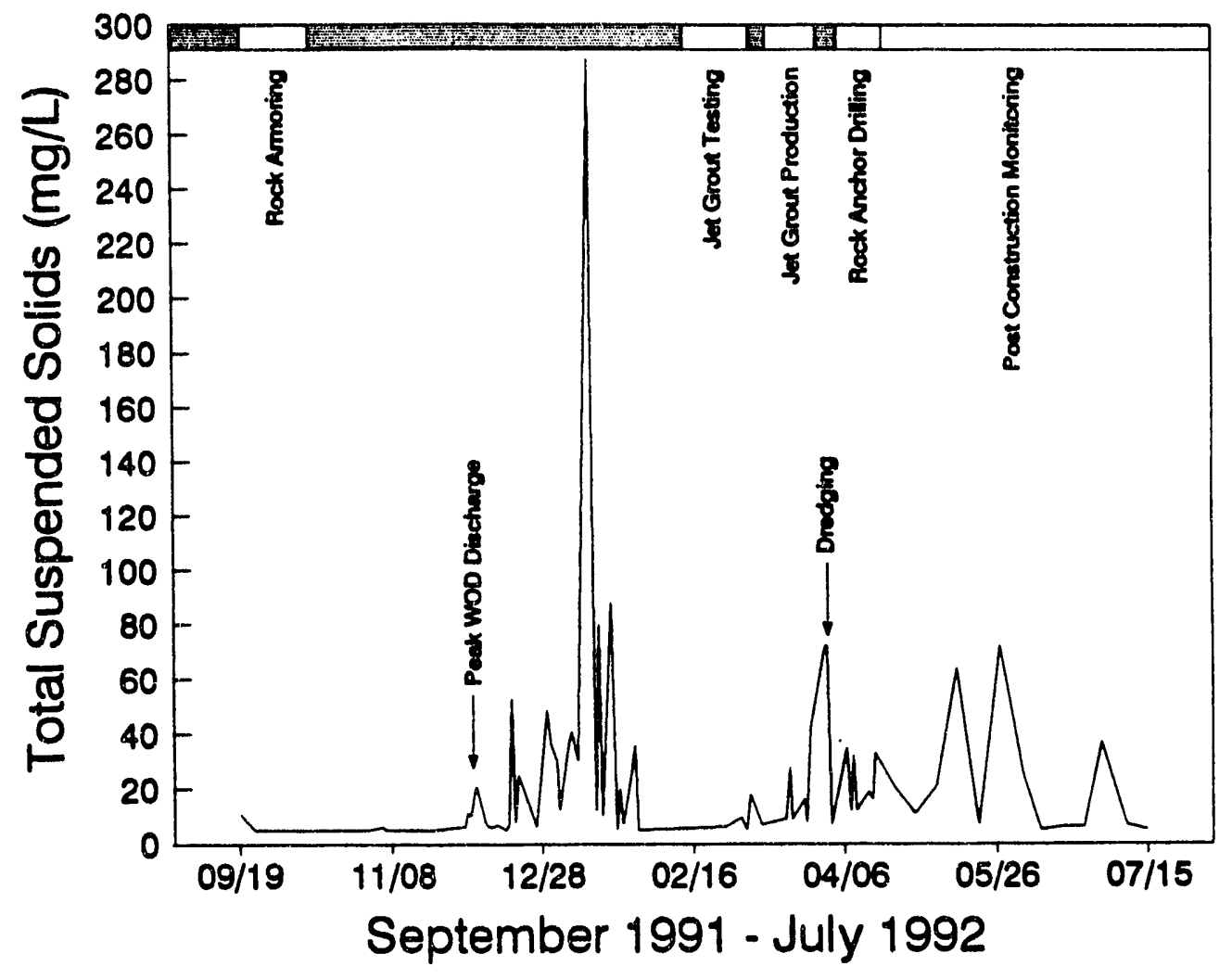

Fig. 4d. Plot of total suspended solids (mg/L) in 24-h composite samples at the K-1513 water intake structure. 


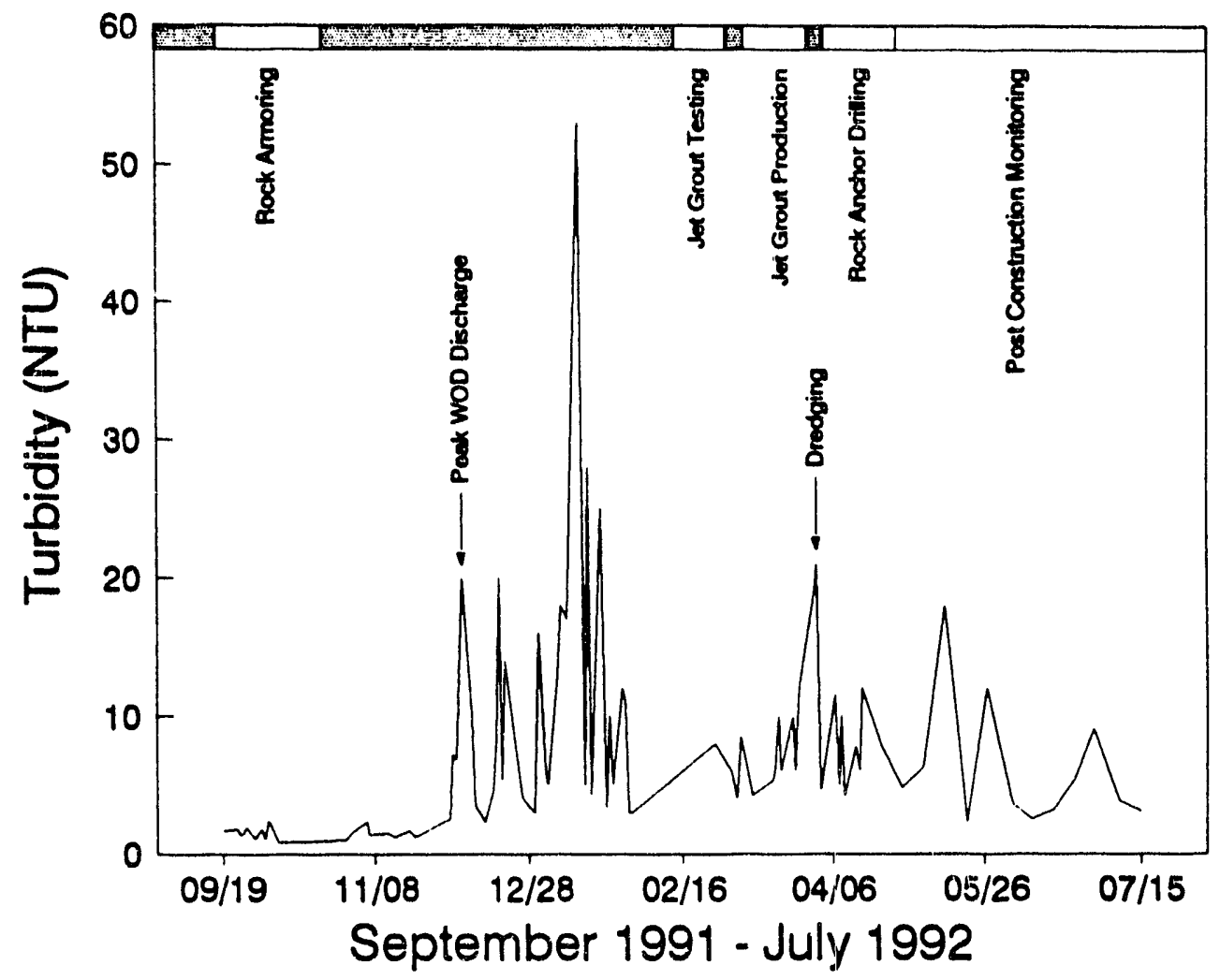

Fig. 4e. Plot of turbidity (NTU) in 24h composite samples at the K-1513 water intake structure. 
Table 3. Water quality sampling results from $24-\mathrm{h}$ composite sampler operations at the K-1513 and WOC mouth stations

\begin{tabular}{lrrrrr}
\hline Analyte & Number & Maximum & Minimum & Mean & Units \\
\hline K-1513 (CRM 14.5) & 109 & 13.8 & -10.8 & 1.3 & $\mathrm{pCi} / \mathrm{L}$ \\
${ }^{137} \mathrm{Cs}$ (total) & 56 & 247.8 & -64.9 & 51.8 & $\mathrm{pCi} / \mathrm{g}$ \\
${ }^{137} \mathrm{Cs}$ (particle-associated) & 90 & 288.0 & 0.0 & 22.3 & $\mathrm{mg} / \mathrm{L}$ \\
TSS & 88 & 55.0 & 0.8 & 8.4 & $\mathrm{NTU}$ \\
Turbidity & & & & & \\
& & & & & \\
WOC mouth (CRM 20.8) & 84 & 170.3 & -11.1 & 16.7 & $\mathrm{pCi} / \mathrm{L}$ \\
${ }^{137}$ Cs (total) & 66 & 4919.5 & -540.6 & 943.3 & $\mathrm{pCi} / \mathrm{g}$ \\
${ }^{137}$ Cs (particle-associated) & 84 & 51.0 & 2.0 & 10.3 & $\mathrm{mg} / \mathrm{L}$ \\
TSS & 85 & 28.0 & 0.8 & 6.1 & $\mathrm{NTU}$ \\
Turbidity & & & & & \\
\hline
\end{tabular}

\subsection{CONTINUOUS WATER QUALITY MONITORING}

Routine water quality monitoring results at the WOC station, including temperature, $\mathrm{pH}$, dissolved oxygen, conductivity, and stage height, are summarized in Table 4 and presented in Fig. 5. All water quality results are found in Appendix C. Data from $\mathrm{pH}$ monitoring will be highlighted in Sects. 3.3.3 and 3.3.5. Copies of calibration records for the water quality monitoring equipment are available upon request.

\subsection{EVENT-SPECIFIC MONITORING}

Monitoring results for the various construction activities are presented below. Reasons for monitoring each project phase are as follows:

- Composite water sample collection was initiated to determine reference conditions for the monitoring project, to provide information regarding the downstream transport of contaminants from WOCE due to both natural flows and construction activities, and to evaluate the short-term effectiveness of the structure (Communication WOCEl070891B.SOW in Ford 1993a).

- Sheet- and H-pile driving operations in the WOCE mouth and subsequent restriction of the flow of water into and out of WOCE had the potential for disturbance, resuspension, and release of contaminated sediments (Communication 081591A.SOW in Ford 1993a).

- Rock armoring of the shoreline to prevent erosion involved the placement of large rocks on the sediment surface and had the potential for disturbance resuspension and release of contaminated sediments (Communication WOCEl091791A.SOW in Ford 1993a). 
- Jet grout construction activities to reinforce the internal base of the sediment-retention structure had the potential to both disturb sediment and release high-pH grout to the surface water. All solutions with a $\mathrm{pH}$ greater than 12.5 , such as grout, are classified and regulated by Resource Conservation and Recovery Act (RCRA) as being corrosive (Communications WOCEl030692A.SAP and WOCEl030992A.SOW in Ford 1993a).

Table 4. Water quality sampling results for the WOC mouth monitoring station

\begin{tabular}{|c|c|c|c|c|}
\hline Parameter & Number & Maximum & Minimum & Average \\
\hline \multicolumn{5}{|c|}{ June 1991} \\
\hline $\begin{array}{l}\text { Temperature }\left({ }^{\circ} \mathrm{C}\right) \\
\text { pH } \\
\text { Dissolved oxygen }(\mathrm{mg} / \mathrm{L})\end{array}$ & $\begin{array}{l}366 \\
366 \\
366\end{array}$ & $\begin{array}{r}22.15 \\
7.15 \\
8.61\end{array}$ & $\begin{array}{r}18.73 \\
5.99 \\
3.89\end{array}$ & $\begin{array}{c}20.40 \\
\text { NA } \\
6.89\end{array}$ \\
\hline \multicolumn{5}{|c|}{ Juhy 1991} \\
\hline $\begin{array}{l}\text { Temperature }\left({ }^{\circ} \mathrm{C}\right) \\
\text { pH } \\
\text { Dissolved oxygen }(\mathrm{mg} / \mathrm{L})\end{array}$ & $\begin{array}{l}353 \\
353 \\
353\end{array}$ & $\begin{array}{r}21.97 \\
6.87 \\
9.13\end{array}$ & $\begin{array}{r}17.42 \\
4.77 \\
6.20\end{array}$ & $\begin{array}{l}18.95 \\
\text { NA } \\
7.72\end{array}$ \\
\hline \multicolumn{5}{|c|}{ August 1991} \\
\hline $\begin{array}{l}\text { Temperature }\left({ }^{\circ} \mathrm{C}\right) \\
\mathrm{pH} \\
\text { Dissolved oxygen }(\mathrm{mg} / \mathrm{L})\end{array}$ & $\begin{array}{l}167 \\
167 \\
167\end{array}$ & $\begin{array}{r}21.33 \\
6.76 \\
6.83\end{array}$ & $\begin{array}{r}18.22 \\
6.17 \\
5.04\end{array}$ & $\begin{array}{c}19.16 \\
\text { NA } \\
5.86\end{array}$ \\
\hline \multicolumn{5}{|c|}{ September 1991} \\
\hline $\begin{array}{l}\text { Temperature }\left({ }^{\circ} \mathrm{C}\right) \\
\mathrm{pH} \\
\text { Dissolved oxygen }(\mathrm{mg} / \mathrm{L})\end{array}$ & $\begin{array}{l}354 \\
354 \\
354\end{array}$ & $\begin{array}{r}21.23 \\
8.35 \\
11.31\end{array}$ & $\begin{array}{r}19.08 \\
5.58 \\
4.98\end{array}$ & $\begin{array}{c}354.00 \\
\text { NA } \\
6.84\end{array}$ \\
\hline \multicolumn{5}{|c|}{ February 1992} \\
\hline $\begin{array}{l}\text { Temperature }\left({ }^{\circ} \mathrm{C}\right) \\
\mathrm{pH} \\
\text { Dissolved oxygen }(\mathrm{mg} / \mathrm{L})\end{array}$ & $\begin{array}{l}238 \\
238 \\
238\end{array}$ & $\begin{array}{r}22.47 \\
9.50 \\
22.08\end{array}$ & $\begin{array}{l}4.99 \\
4.99 \\
6.38\end{array}$ & $\begin{array}{l}9.75 \\
\text { NA } \\
14.78\end{array}$ \\
\hline \multicolumn{5}{|c|}{ March 1992} \\
\hline $\begin{array}{l}\text { Temperature }\left({ }^{\circ} \mathrm{C}\right) \\
\mathrm{pH} \\
\text { Dissolved oxygen }(\mathrm{mg} / \mathrm{L})\end{array}$ & $\begin{array}{l}244 \\
244 \\
244\end{array}$ & $\begin{array}{r}14.38 \\
8.84 \\
18.57\end{array}$ & $\begin{array}{r}9.20 \\
7.37 \\
10.92\end{array}$ & $\begin{array}{c}11.94 \\
\text { NA } \\
15.88\end{array}$ \\
\hline \multicolumn{5}{|c|}{ April 1992} \\
\hline $\begin{array}{l}\text { Temperature }\left({ }^{\circ} \mathrm{C}\right) \\
\mathrm{pH} \\
\text { Dissolved oxygen }(\mathrm{mg} / \mathrm{L})\end{array}$ & $\begin{array}{l}205 \\
205 \\
205\end{array}$ & $\begin{array}{r}16.82 \\
8.96 \\
18.44\end{array}$ & $\begin{array}{r}9.65 \\
7.83 \\
10.25\end{array}$ & $\begin{array}{c}12.57 \\
\text { NA } \\
16.09\end{array}$ \\
\hline
\end{tabular}



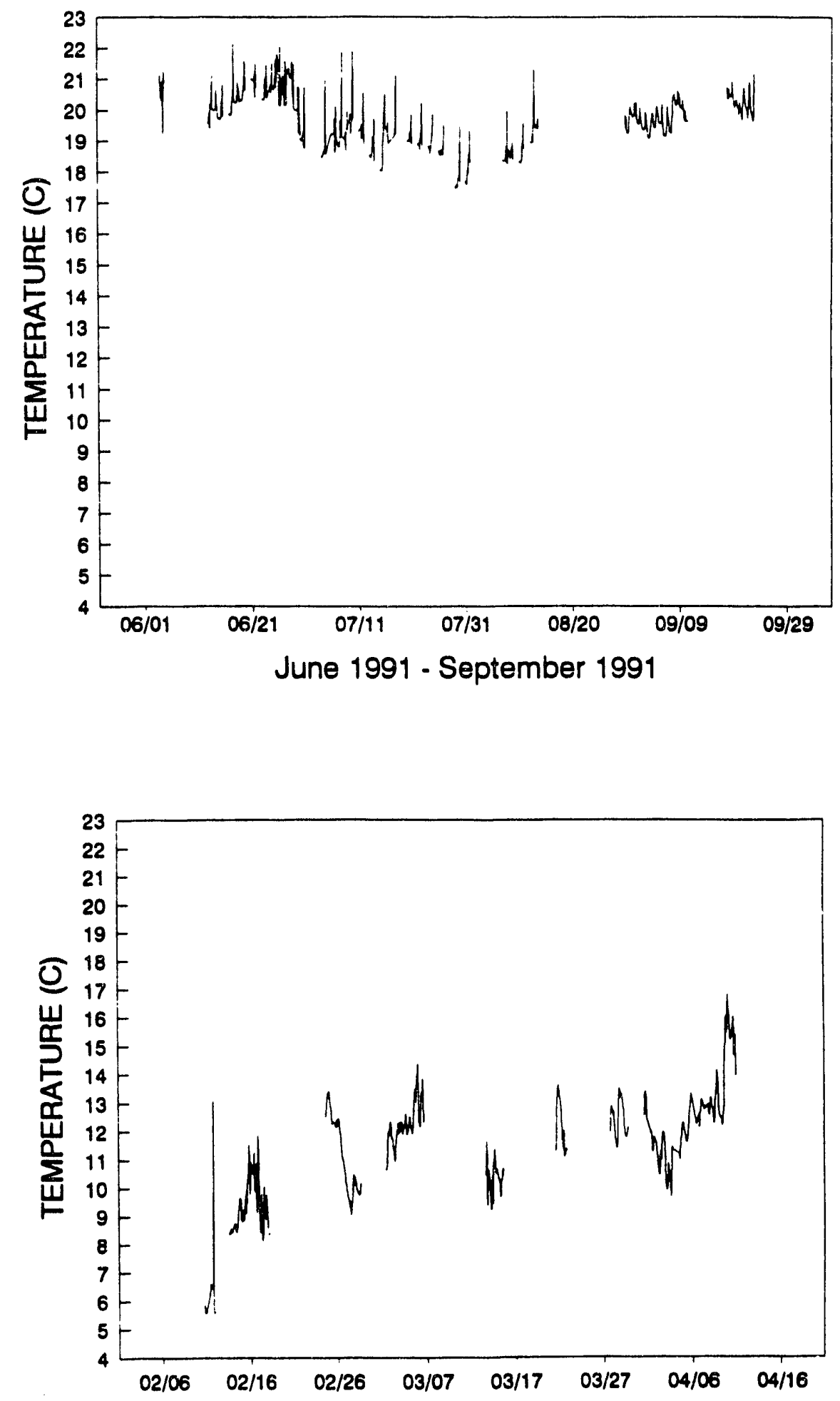

February 10, 1992 - April 10, 1992

Fig. 5a. Continuous plot of temperature for the water quality monitoring station at the mouth of White Oak Creek. 


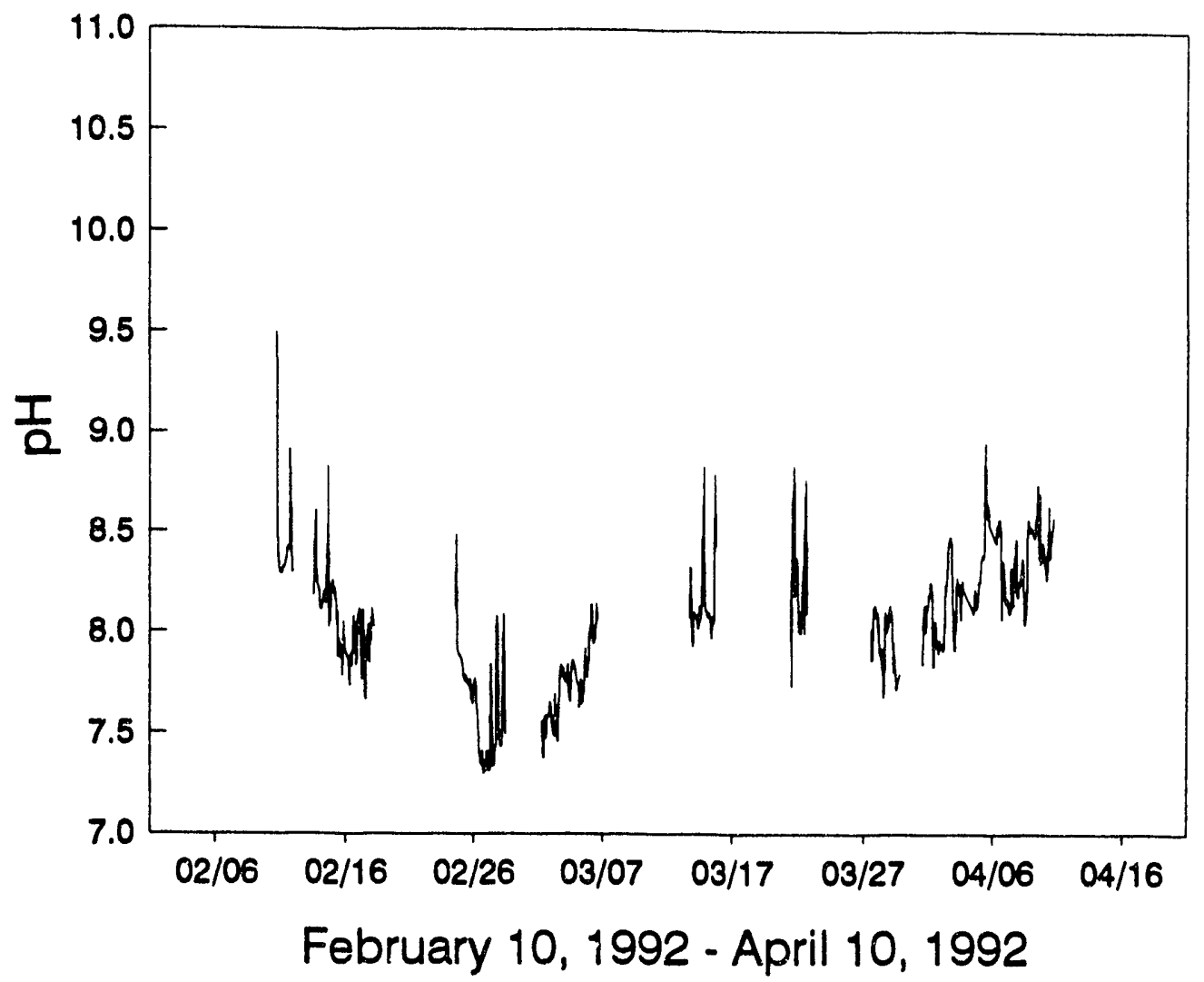

Fig. 5b. Continuous plot of pH for the water quality monitoring station at the mouth of White Oak Creek. 

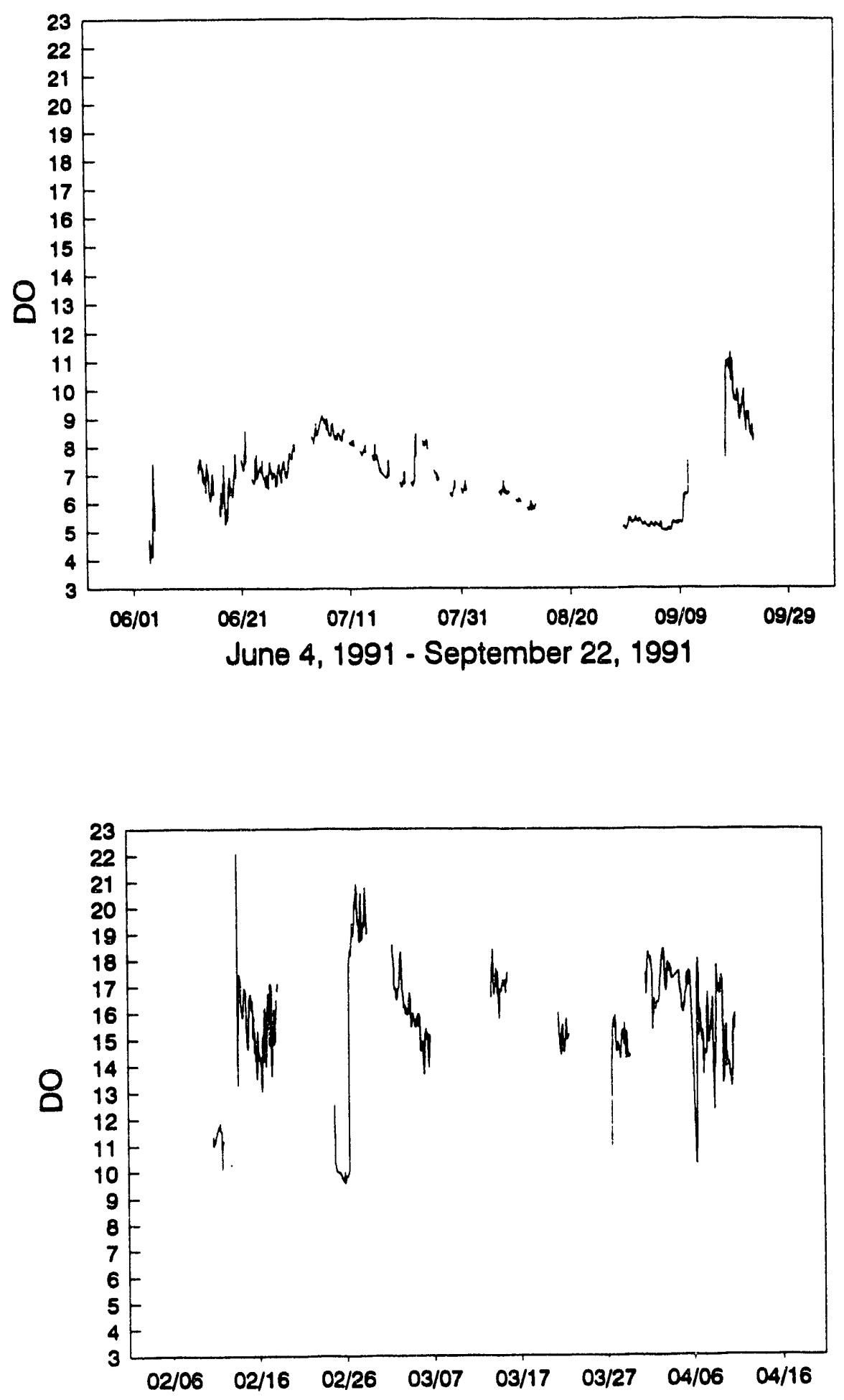

February 10, 1992 - April 10, 1992

Fig. 5c. Continuous plot of dissolved axygen for the water quality monitoring station at the mouth of White Oak Creek. 

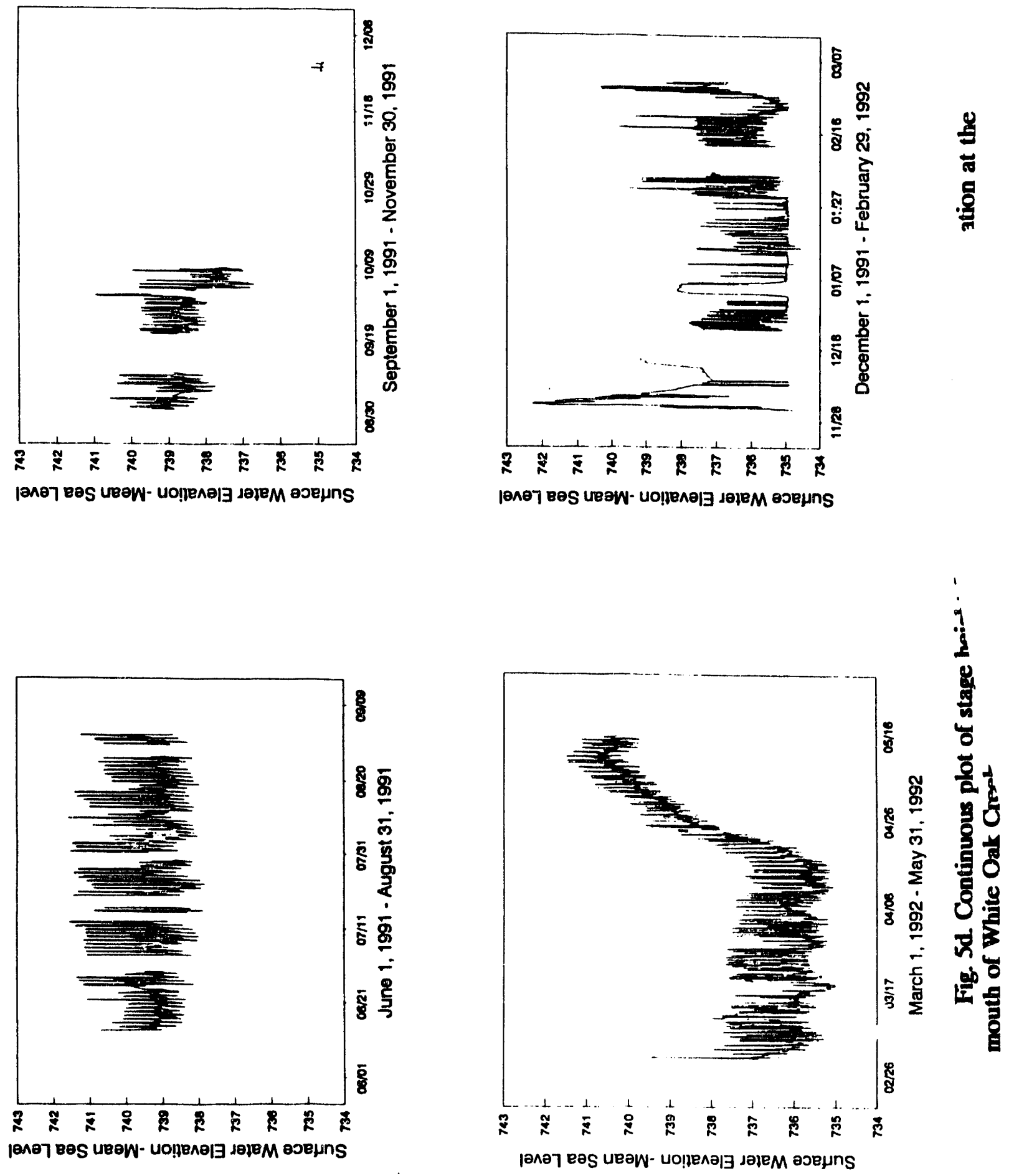
Additional construction activities including the following were monitored:

- Surface sediment relocation within the coffer cell was performed to provide adequate space for concrete cap installation following jet grout completion. Monitoring focused on sediment disturbance and transport downstream due to dredge operations (Communication WOCEl032592A in Ford 1993a).

- Concrete cap installation had the potential for release of high-pH cement (RCRA corrosive) materials; monitoring followed the same guidance as that for $\mathrm{pH}$ monitoring of jet grout operations (Communication WOCE|030992A.SOW in Ford 1993a).

- Rock anchor drilling and installation had a great potential for disturbance of surface sediments; suspended materials collection followed the same guidance as given for dredging (Communication WOCE 032592A in Ford 1993a).

Rock anchor drilling and installation was the last construction activity monitored for specific sediment-disturbing activities. Monitoring continued for $3 \frac{1}{2} 2$ months after construction activity ceased.

Results from each phase of the construction project requiring on-site, real-time sample and data collection are presented below. All event-specific monitoring data are found in Appendix D.

\subsubsection{Sheet Pile Installation}

Sheet pile installation in the WOC streambed occurred from August 20 through August 27, 1991. Discharge rates from both the WOD (Fig. 2) and Melton Hill Dam were regulated during the final stages of sheet pile installation to minimize flows and flow changes at the mouth of WOC. Increases in contaminant concentrations were not observed at the WOC mouth during pile-driving operations. All water quality parameters were within values previously observed for this season and were comparable with WOD data for the same time frame (Kornegay et al. 1992 and Appendix B). Contaminant data collected in conjunction with pile driving are presented in Appendix $\mathrm{D}$.

\subsubsection{Rock Armoring}

Rock armoring of the shoreline was conducted from September 18 through October 8, 1991 , to reinforce the banks of rivers and creeks in the vicinity of the sediment-retention structure. Small-scale turbidity plumes were observed by sampling teams during rock armoring of the shoreline at the coffer cell; however, contaminant concentrations did not increase at the WOC mouth in association with these operations. All water quality parameters were within values previously observed for this season and were comparable with WOD data for the same time frame (Kornegay et al. 1992 and Appendix B). Data collected in conjunction with rock armoring are presented in Appendix D.

\subsubsection{Jet Grouting}

Jet grout activities were conducted from February 10 through March 25, 1992. In-stream $\mathrm{pH}$ increased at the mouth of WOC in conjunction with jet groutilig activities (Figs. 3c, 5b, 
6, Table 5, Appendix D). Twenty-four hour composite sample $\mathrm{pH}$ values never exceeded $\mathrm{pH} 9$ during grouting activities (Fig. 3c). Manual recording (Fig. 5b) and continuous monitoring at the site yielded $\mathrm{pH}$ maxima near 10 during jet grouting. A single construction-related release resulted in a pH peak of approximately 10.6 for surface waters (Fig. 6). Increases in ${ }^{137} \mathrm{Cs}$ activity during jet grouting in the coffer cell were observed at the WOC mouth (Figs. 3a and 3b). Temperature and dissolved oxygen parameters were within seasonally expected normal values. Increases in ${ }^{137} \mathrm{Cs}$ activity associated with jet grouting were observed at the WOC mouth. No increases in $\mathrm{pH}$ or ${ }^{137} \mathrm{Cs}$ activity were observed at $\mathrm{K}-1513$ in association with jet grouting activities (Fig. 4).

Table 5. Summary of parameters, range, and number of observations for samples collected during construction of the sediment retention structure at WOCK 0.0

(Data were collected from February 10 through March 25, 1992.)

\begin{tabular}{lccccc}
\hline Analyte & Number & Maximum & Minimum & Mean & Units \\
\hline $\begin{array}{l}{ }^{137} \mathrm{Cs} \text { (particle- } \\
\text { associated) }\end{array}$ & 22 & 3186.4 & 585.7 & 1858.6 & $\mathrm{pCi} / \mathrm{g}$ \\
& 58 & 324.4 & -10.8 & 110.0 & $\mathrm{pCi} / \mathrm{L}$ \\
${ }^{137} \mathrm{Cs}$ (total) & 22 & 22.6 & 6.2 & 14.3 & $\mathrm{mg} / \mathrm{L}$ \\
$\begin{array}{l}\text { Total suspended } \\
\text { solids }\end{array}$ & & & & & \\
\hline
\end{tabular}

\subsubsection{Dredging}

Dredging to remove excess sediment from the sediment-retention structure was conducted on March 26 and April 1, 1992. Turbidity plumes were observed in conjunction with dredging activities. Resuspension and transport of contaminated sediment was evident in both the composite sampler and grab samples collected in conjunction with dredging (Figs. 3a and 3b; Tables 3 and 6). Composite samples indicated that ${ }^{137} \mathrm{Cs}$-contaminated sediment had been released.

Twenty-four-hour composite sampler results for ${ }^{137} \mathrm{Cs}$ activity at both the WOC mouth and K-1513 monitoring stations increased in conjunction with sediment dredging (Table 3). At the WOC station, particle-assusiated ${ }^{137} \mathrm{Cs}$ values for dredging activity at WOC were elevated by one to two orders of magnitude $\left[170 \mathrm{pCi} / \mathrm{L}\right.$ total ${ }^{137} \mathrm{Cs}$ and $2900 \mathrm{pCi} / \mathrm{g}$ dry weight suspended solids of ${ }^{137} \mathrm{Cs}$ (Appendix A)] relative to nonconstruction reference levels (Appendix A). Values for $\mathrm{K}-1513$ were also elevated for the time frame, though they were much lower than those in WOC [range 1 to $10 \mathrm{pCi} / \mathrm{L}$ for total ${ }^{137} \mathrm{Cs}$ activity, maximum particle-associated ${ }^{137} \mathrm{Cs}=223 \mathrm{pCi} / \mathrm{g}$ (Appendix A)].

Grab sample results for ${ }^{137} \mathrm{Cs}$ values in surface-water grab samples collected in the immediate vicinity of dredging-se timent plumes in bulk samples ranged from 10 to $324 \mathrm{pCi} / \mathrm{L}$, while particle-associated values ringed from 800 to $7150 \mathrm{pCi} / \mathrm{g}$ (Table 6 and Appendix D). 


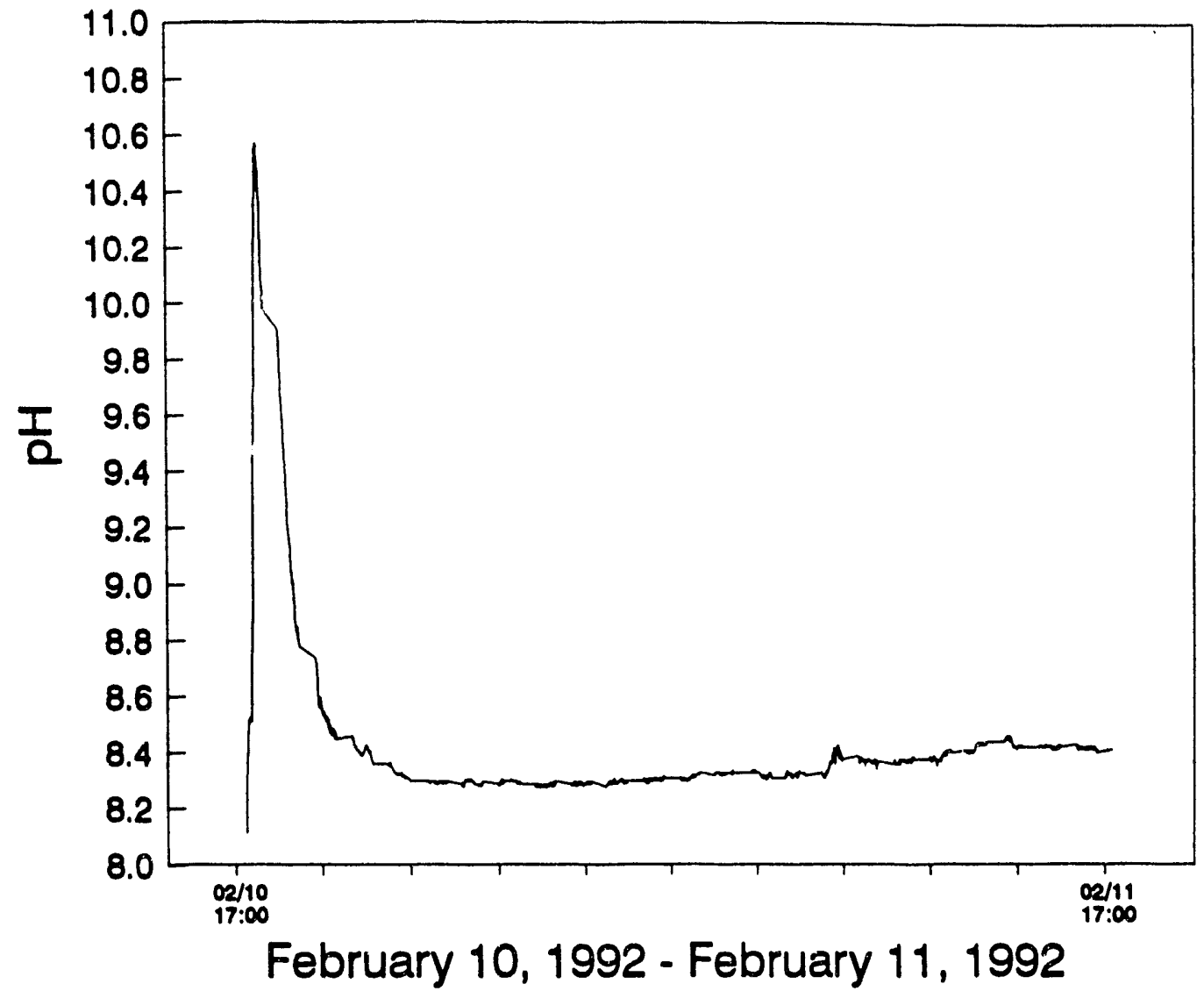

Fig. 6. Continuous plot of $\mathrm{pH}$ values for grout released to surface waters. 
Table 6. Summary of parameters, range, and number of observations for samples collected during sediment-dredging activities associated with construction of the sediment retention structure at WOCK 0.0

(Data were collected on March 26 and April 1, 1992.)

\begin{tabular}{|c|c|c|c|c|c|}
\hline Analyte & Number & Maximum & Minimum & Mean & Units \\
\hline $\begin{array}{l}{ }^{137} \mathrm{Cs} \text { (particle- } \\
\text { associated) }\end{array}$ & 13 & 7148.4 & 0.0 & 2815.4 & $\mathrm{pCi} / \mathrm{g}$ \\
\hline${ }^{137} \mathrm{Cs}$ (total) & 8 & 324.4 & 10.8 & 90.6 & $\mathrm{pCi} / \mathrm{L}$ \\
\hline $\begin{array}{l}\text { Total suspended } \\
\text { solids }\end{array}$ & 13 & 47.0 & 3.4 & 23.9 & $\mathrm{mg} / \mathrm{L}$ \\
\hline
\end{tabular}

\subsubsection{Concrete Cap Installation}

Concreie caps were installed in the sediment-retention structure or Viarch 27 and April 5, 1992. Radiological data fron: composite sampies sollested during concrete cap construction do not indicate increased ${ }^{137} \mathrm{Cs}$ levels assoriated with these activities. No increases in pH or contaminant concentrations associated writh concrete cap installation were observed at the WOC mouth (Appendix D). All water quality parameters viere within seasonally expected normal values.

\subsubsection{Rock Archor Drilling}

Drilling of rock anchors was conducted in the sedimerit-retention structure from April 4 through April 10, 19:2. Cesium-137 activity in 24-h composite samples at the WOC mouth and $\mathrm{K}-1513$ increased in conjunction with this phase of construction (Figs. 3a, 3b, 4a, and 4b; Table 7); activities were also elevated in grab samples collected at the WOC mouth. The maximum total ${ }^{137} \mathrm{Cs}$ level for 24 -h composites at WOC was approximately $2400 \mathrm{pCi} / \mathrm{L}$ (Appendix A), with maximum particle-associated ${ }^{137} \mathrm{Cs}$ values of $4900 \mathrm{pCi} / \mathrm{g}$ (Appendix A). Composite sampler maximum values for $\mathrm{K}-1513$ were $1 \mathrm{pCi} / \mathrm{L}$ total ${ }^{137} \mathrm{Cs}$ and $250 \mathrm{pCi} / \mathrm{g}$ for particle-associated ${ }^{137} \mathrm{Cs}$ samples (Appendix A). Grab samples collected directly from resuspended sediment plumes at the WOC mouth ranged from below background ( $1 \mathrm{pCi} / \mathrm{L}$ ) to $2400 \mathrm{pCi} / \mathrm{L}$ total ${ }^{13} \mathrm{Cs}$. Particle-associated ${ }^{137} \mathrm{Cs}$ values ranged from 400 to $6700 \mathrm{pCi} / \mathrm{g}$ (Appendix D).

\subsubsection{Postconstruction Monitoring}

Composite sample collection continued for $3 \frac{1}{2}$ months following the conclusion of construction. Values for total ${ }^{137} \mathrm{Cs}$, total suspended solids, and turbidity dropped following final completion of the coffer-cell structure (Figs. 3a, 3b, 4a, and 4b; Appendix A). Collection of 24-h composite samples for assessment of particle-associated ${ }^{137} \mathrm{Cs}$ was discontinued shortly after construction was complete. Though detectable quantities of radionuclides were observed at both monitoring stations, these values were within the range of measured values in water 
released from WOL over the same time period (Appendix B) and were comparable to values reported in previous years (Kornegay et al. 1990, 1991, and 1992).

Table 7. List of parameters, range, and number of observations for samples collected during rock anchor drilling activities associated with construction of the sediment retention structure at WOCK 0.0

(Data were collected from April 6 through April 10, 1992.)

\begin{tabular}{lccccc}
\hline Analyte & Number & Maximum & Minimum & Mean & Units \\
\hline $\begin{array}{l}{ }^{137} \mathrm{Cs} \text { (particle- } \\
\text { associated; }\end{array}$ & 21 & 6735.2 & 407.4 & 3144.3 & $\mathrm{pCi} / \mathrm{g}$ \\
& 10 & 2378.6 & -10.8 & 595.7 & $\mathrm{pCi} / \mathrm{L}$ \\
${ }^{137} \mathrm{Cs}$ (total) & & & & & \\
Total suspended solids & 21 & 113.0 & 5.7 & 38.7 & $\mathrm{mg} / \mathrm{L}$ \\
\hline
\end{tabular}




\section{DISCUSSION}

Monitoring results for specific construction activities are discussed in the following sections. Specific construction project-driven objectives for monitoring appear in Sect. 3.3.

\subsection{COMPOSITE SAMPLING}

Composite sampler operations at or near the WOC mouth station were conducted from June 1991 through July 1992 (Table 1). During periods when no construction occurred, the sampler at this station did not function because of cold temperatures and low water levels (Table 3). Composite sampler operations at the K-1513 station (the K-25 Site water intake structure at CRM 14.5) began in September 1991 and continued through July 1992; samples from this station were obtained regardless of lake-level fluctuations. Composite sample $\mathrm{pH}$ values were collected from February through July 1992 and are discussed in Sect. 4.3.3. The frequency of sample collection from either the WOC or K-1513 stations depended upon the construction activity.

Turbidity and TSS concentrations at both stations varied greatly with seasonal changes in ambient suspended solids concentrations in the Clinch River (Figs. 3d, 3e, 4d, and 4e). During the early fall (September, October, and early November 1991), TSS values rarely rose above the detection limit of $5 \mathrm{mg} / \mathrm{L}$, with turbidity values below 3 NTU. By late November, values had begun to rise; they varied between 6 and $50 \mathrm{mg} / \mathrm{L}$. TSS peaked at over $250 \mathrm{mg} / \mathrm{L}$ (Table 3 and Appendix A) during early January, dropping gradually back to the 10 - to $50-\mathrm{mg} / \mathrm{L}$ range in February and March. TSS dropped to or below the limits of detection by early May and remained low through the summer, except when summer storms briefly (episodically) drove suspended solids values up again. No increases attributable solely to construction activities were observed in 24-h composite samples. Neither did analytical results for sample $\mathrm{pH}$, initiated in February for 24-h composite samples, indicate observable impacts to surface waters from construction activities.

Cesium-137 is a highly particle-reactive radionuclide with a particle-to-water distribution coefficient $\left(\mathrm{K}_{\mathrm{D}}=\right.$ concentration per kilogram of particles divided by concentration per liter of water) of approximately $10^{5}$ (Olsen et al. 1992). The WOD and WOC mouth water samples show low but measurable concentrations of ${ }^{137} \mathrm{Cs}$ leaving WOC during normal flow conditions (Appendixes B and D). These values for the WOC mouth dropped immediately following closure of the coffer-cell wall and stayed low during the early fall of 1991 regardless of construction activities (Figs. 3a and 3b). Cesium-137 levels at the WOC mouth rose with increasing late fall rainstorms, runoff, and increasing TSS levels, all due to normal seasonal changes in water conditions. Similar episodic patterns in discharge, water quality, and contaminant concentrations were observed at the WOD sampling station (Fig. 2 and Appendix B). Throughout December, January, and part of February, the ${ }^{137} \mathrm{Cs}$ values at the WOC mouth remained low, normally below detection. The peak total ${ }^{137} \mathrm{Cs}$ value for $\mathrm{K}-1513$ was observed during December 1991 coincident with a high-volume discharge from WOD (Figs. 2 and 4a; Table 3; Appendix A). With this exception, data from the K-1513 station from September 1991 through February 1992 showed little or no detectable ${ }^{137} \mathrm{Cs}$ contamination at the K-25 Site water intake despite high particle loads in the Clinch River (Fig. 4). This implies that (1) a majority of the suspended-particle load in the Clinch River comes from 
sources not contaminated with ${ }^{137} \mathrm{Cs}$ upstream in the Clinch River watershed, and (2) for most time periods, WOC discharge to the Clinch River is diluted sufficiently so as to be undetectable in surface water samples $10 \mathrm{~km}$ downstream.

Radiological data from 24-h composite samples collected from the WOC station during jet grouting and concrete cap construction indicate that there were no increases in ${ }^{137} \mathrm{Cs}$ levels associated with these activities over levels measured upstream from WOD (Table 5; Fig. 4; Appendix A). Cesium-137 results at the K-1513 station were below the quantitation limit. However, there were marked increases in 24-h composite radiological results coincident with sediment dredging and rock anchor drilling (Figs. 3a, 3b, 4a, and 4b; Appendix D). At the WOC station, maximum values during dredging activities were $170 \mathrm{pCi} / \mathrm{L}$ and $2900 \mathrm{pCi} / \mathrm{g}$ for total and particle-associated ${ }^{137} \mathrm{Cs}$, respectively, greatly elevated relative to all previous 24 -h composite sample observations at the WOC mouth. The values for $\mathrm{K}-1513$ were also elevated for the time frame, though they were lower than observed at the WOC mouth; K-1513 maximum values were $10 \mathrm{pCi} / \mathrm{L}$ and $1400 \mathrm{pCi} / \mathrm{g}$ total ${ }^{137} \mathrm{Cs}$ and particle-associated ${ }^{137} \mathrm{Cs}$ respectively. These elevated $24 \mathrm{~h}$ composite results may be a function of low discharge rates from White Oak and Melton Hill dams, resulting in low dilution ratios for contaminants. Flow volumes were maintained at a low level during dredge operations to reduce sediment scouring (Fig. 2). This elevation in ${ }^{137} \mathrm{Cs}$ illustrates the importance of minimizing to the extent practical the disturbance of these sediments.

Levels of particle-associated radiological contaminants in water dropped to below detection limits at both the K-1513 and WOC mouth stations following completion of the sediment-retention structure. Values were comparable with those observed during nonconstruction monitoring, following the establishment of surface sediment control in September 1991 (Figs. 3a, 3b, 4a, and 4b). There were still episodes of ${ }^{137} \mathrm{Cs}$ release to the Clinch River, though these may be related to sediment resuspension and transport from WOL through WOCE and into the Clinch River during elevated discharge (Fig. 2 and Appendix B).

QAVC data collected in conjunction with 24-h composite samples indicate that the analytical standard errors for total suspended particles, turbidity, and $\mathrm{pH}$ results were $10 \%$ or less.

\subsection{CONITNUOUS WATER QUALTY MONITORING}

Routine water quality monitoring results at the WOC station indicated normal seasonal shifts in temperature, pH, dissolved oxygen, and conductivity during the course of this monitoring project (Fig. 5; Table 4; Appendix C). Monitoring of $\mathrm{pH}$ during jet grout and concrete cap construction is discussed in Sects. 4.3.3 and 4.3.4. Stage height data collected throughout this project illustrate the impact of routine reservoir operations on water elevations at the WOC mouth (Fig. Se). As noted in Sect. 2.1.2, continuous nephelometric monitoring at the site was discontinued prior to construction. 


\subsection{EVENT-SPECIFIC MONITORING}

Results from each phase of the construction project requiring on-site, real-time sample and data collection are discussed here. The objectives for real-time monitoring (Sect. 1.3) focused on construction-related contaminant releases and determined the data and sample collection for each construction event. All event-specific monitoring data are found in Appendix D.

\subsubsection{Sheet Pile Installation}

Pile-driving activities conducted in August 1991 and the resulting restriction of the WOC channel increased the potential for scouring of contaminated sediments out of the streambed. These activities were monitored by close-interval sampling from a boat in the embayment mouth as well as through composite sampler monitoring (Communication 081591A.SOW in Ford 1993a). Increases in contamination at either the WOCE or the K-1513 stations would be indicative of sediment scour. Water releases from White Oak and Melton Hill dams were regulated to minimize flows during the final stages of this installation process.

No increases in contaminant resuspension and transport into the Clinch River associated with pile-driving operations were observed for any monitored parameters (Appendix D). None of the monitored water quality variables were affected by the construction activity. Water flow restriction from both dams had no observable impact upon contaminant concentrations.

\subsubsection{Rock Armoring}

Placement of rock to armor the shoreline was performed from mid-September through early October 1991 . Erosion prevention by rock armoring involves the placement of large rocks on the sediment surface using a clamshell dredge. This activity had a great potential to disturb and release contaminated sediments (Communication WOCEl091791A.SOW in Ford 1993a). These activities were completed prior to grout reinforcement and rock anchor drilling, out of the original construction sequence due to construction delays (Table 2; Kimmel and Ford 1991). Resuspension events resulting from the displacement of potentially contaminated sediments by armoring were monitored by close-interval sampling from a boat as well as through composite sampler monitoring. Samples collected from the plume were analyzed for particle-associated and total ${ }^{137} \mathrm{Cs}$, TSS, and turbidity.

Rock armoring of the shoreline resulted in generation of small-scale observable plumes of sediment in lower WOCE and the Clinch River; however, these plumes dissipated quickly and did not result in changes in any monitored parameter at either monitoring station (Appendix D).

\subsubsection{Jet Grouting}

The injection of grout/water mixtures to reinforce the foundation of the sedimentretention structure at the sediment/bedrock interface (jet grouting) was performed during February and March 1992 (Communication WOCEl030992A.SOW in Ford 1993a). Concentrated grout mixtures such as are used for jet grouting have a $\mathrm{pH}$ of approximately 12, which places them in the range of RCRA corrosive materials. Monitoring activities during jet 
grouting focused upon potential sediment disturbance and the possible release of high-pH grout directly to surface waters in violation of Tennessee state water quality standards (Communications WOCEl030692A.SAP and WOCEl030992A.SOW in Ford 1993a). Release of grout-contaminated materials was indicated by an observed suspended solids plume accompanied by increased surface water $\mathrm{pH}$. Continuous monitoring of in-stream pH occurred whenever jet grout activity was in progress. Samples were analyzed for particle-associated and total ${ }^{137} \mathrm{Cs}$, TSS, turbidity, and pH (Table 5 and Appendix D). Cesium-137 activity in grab samples at the WOC station, comparable with that from WOD (Appendix B), was observed in association with jet grouting at the WOC mouth.

High-pH grout releases during the jet grouting activity were not evident from routine analytical requests for $24 \mathrm{~h}$ composite samples, which yielded maximum values below $\mathrm{pH} 9$ (Fig. 3c). However, monitoring data from the continuous recording device at the site (Figs. 5b and 6) closely tracked $\mathrm{pH}$ increases related to the release of grout. A single constructionrelated release resulted in a $\mathrm{pH}$ peak of approximately 10.6 for surface waters (Fig. 6). The impact to surface water $\mathrm{pH}$ from this event dissipated within $4 \mathrm{~h}$. Additional high-pH grout releases resulted in momentary increases to over $\mathrm{pH}$ 9.7, though such releases were rare and short-lived. Occurrence reports detailing the impact of construction activities on surface waters were filed when in-stream $\mathrm{pH}$ values rose above 10 for any length of time.

\subsubsection{Dredging}

Surface sediment relocation within the coffer cell was performed in order to provide adequate space for concrete cap installation following jet grout completion (Communication WOCEl032592A.SOW in Ford 1993a). A small amount of surface sediment relocation was necessitated by sediment upheaval resulting from jet grout operations. Sediment relocation was accomplished with a clamshell dredge. Monitoring activities focused upon sediment disturbance and transport downstream due to dredge operations. Water discharge from both White Oak (Fig. 2) and Melton Hill dams was minimized during the sediment relocation process.

Between a few centimeters and $0.5 \mathrm{~m}$ of surface sediment, with an estimated volume of $2.1 \mathrm{~m}^{3}$, was relocated. This activity resulted in resuspension and transport of contaminated sediment out of the coffer cell. Release of ${ }^{137} \mathrm{Cs}$-contaminated sediments by this small relocation was evident in both the 24-h composite and on-site grab samples collected in conjunction with dredging (Figs. $3 a$ and $3 b$; Table 6). At the WOC station, grab samples from real-time monitoring yielded values from 800 to $7150 \mathrm{pCi}$ of ${ }^{137} \mathrm{Cs}$ per gram of suspended solids, values comparable with surface sediments in the vicinity of the dredging activity (Blaylock et al. 1993). Twenty-four-hour composite values for particle-associated ${ }^{137} \mathrm{Cs}$ were also greatly elevated, with maximum particle-associated ${ }^{137} \mathrm{Cs}$ of $2900 \mathrm{pCi} / \mathrm{g}$. Values for 24-h composite sample collection at $\mathrm{K}-1513$ were also high for the time frame, though they were lower than those upstream (range 1 to $10 \mathrm{pCi} / \mathrm{L}$ for total ${ }^{137} \mathrm{Cs}$ activity; maximum particleassociated ${ }^{137} \mathrm{Cs}$ was $\left.1400 \mathrm{pCi} / \mathrm{g}\right)$.

Though construction was designed to avoid such overt sediment disturbance, relocation became necessary to ensure the structural integrity of the coffer cell. These data reinforce the importance of minimal sediment disturbance during construction, suggesting that large quantities of particle-associated ${ }^{137} \mathrm{Cs}$ would have been released if conventional excavation construction techniques had been used. 


\subsubsection{Concrete Cap Installation}

Monitoring for concrete cap installation followed the same scheme as monitoring for jet grouting. No sediment disturbance was anticipated or observed with the concrete cap installation. Installation was performed in two stages to allow proper curing of the concrete prior to inundation; the side cells were poured on March 26,1992, and the center cell was poured on April 5, 1992. Water discharge from White Oak (Fig. 2) and Melton Hill dams was minimized during this installation process.

Site sample collection was tied to the observation of elevated $\mathrm{pH}$ values or turbidity plumes; composite samples were collected in conjunction with the March 26 work only. No increases in $\mathrm{pH}$ or in cement-related plumes were observed in conjunction with cap installation. Unlike jet grouting, there were no increases in ${ }^{137} \mathrm{Cs}$ associated with concrete cap installation (Appendix D).

\subsubsection{Rock Drilling}

Rock anchor drilling and installation was the last construction activity monitored for sediment disturbance. Rock anchor construction involved use of a drill rig over preformed holes in the concrete cap to drill through the sediment and $20 \mathrm{ft}$ into bedrock to install the rock anchors. Release of contaminated sediment during rock anchor drilling had not been considered as a possibility in initial monitoring plans (Kimmel and Ford 1991). The bedrock encountered was fractured, necessitating the high-pressure injection of a fluid through the drill rod to keep the drill bit from becoming fouled; attempts to keep the drill clean and to complete the rock anchor holes with cleaning fluids other than air failed. The possibilities for turbulence and sediment disturbance were not recognized until late in the project when the drilling of a test hole resulted in significant resuspension of surface sediments within the coffer cell. Because of previous delays in the project, there was not time to consider other rock anchor drilling alternatives. Air was injected through the drill stem under high pressure, exiting through the drill bit. After leaving the cutting head, the pressurized air forced its way back to the surface. These high-pressure streams of air effectively resuspended surface sediments outside of the coffer-cell structures both upstream in WOCE and downstream at the WOC mouth.

As reported in Sect. 3.3.6, ${ }^{137} \mathrm{Cs}$ activities in 24-h composite samples from both monitoring stations increased during rock anchor drilling (Figs. 3a, 3b, 4a, and 4b; Table 7). Cesium-137 activities were also elevated for water grab samples collected at the WOC mouth. The maximum ${ }^{137} \mathrm{Cs}$ values for both composite and grab samples are presented in Sect. 3.3.6. Sediment disturbance resulted from streams of air that followed fractures in the bedrock to reach the surface sediment. As with the sediment relocation activity (Sect. 4.3 .4 ), ${ }^{137} \mathrm{Cs}$ levels in water samples were similar to those observed on WOCE surface sediments throughout the embayment (Blaylock et al. 1993). Contaminant levels in 24-h composites for K-1513 were also elevated, with the maximum particle-associated ${ }^{137} \mathrm{Cs}$ value observed during rock anchor drilling (Table 3).

\subsection{Postconstruction Monitoring}

Short-term monitoring conducted after completion of the sediment-retention structure indicates a decrease in ${ }^{137} \mathrm{Cs}$ in the Clinch River over previous years, given similar input to 
WOCE from WOD. This suggests that the structure has effectively created a zone of quiescent water, allowing particle-associated contaminants to settle out of the water column.

Values for total ${ }^{137} \mathrm{Cs}$, TSS, and turbidity dropped following final completion of the sediment-retention structure (Figs. 3a, 3b, 4a, and 4b; Appendix A). Though detectable quantities of radionuclides were observed at both monitoring stations, these values were within the ranges of those released from WOD over the same time period (Appendix B) and comparable with those reported previously (Kornegay et al. 1992). 


\section{CONCLUSIONS}

Construction of the coffer-cell structure at the WOC mouth was performed with a minimal amount of sediment disturbance as compared with conventional excavation techniques. However, the high-pressure injection of air to prevent the rock anchor drill bit frong fouling resuited in the disturbance, resuspension, and transport of contaminated sediment into the Clinch River and downstream. This impact dissipated when rock anchor drilling was completed.

Short-term postconstruction monitoring indicates a decrease in ${ }^{137} \mathrm{Cs}$ in the Clinch River compared with previous years, given similar input to WOCE from WOL. This suggests that the structure has effectively created a zone of quiescent water, allowing particle-associated contaminants to settle out of the water column.

Complete evaluation of the effectiveness of this structure will require more thorough investigation, particularly regarding the impact of high-flow storm events on particle resuspension and transport within and through the embayment. These longer term studies will be carried out by the WAG 2 ER Project, in coordination with the Clinch River ER Program. 


\section{SUMMARY}

Water quality monitoring activities, focused on the detection of resuspended sediments in the Clinch River, were conducted in conjunction with the WOCE time-critical CERCLA removal action to construct a sediment-retention coffer-cell structure at the mouth of WOC. Samples were collected by use of both a 24-h composite sampler and real-time water grab sampling of sediment plumes generated by the construction activities.

Surface water radiological data collected prior to coffer-cell closure were used to gauge the effectiveness of the coffer-cell structure at the WOC mouth. These data showed low but measurable quantities of ${ }^{137} \mathrm{Cs}$ leaving the embayment under normal flow conditions.

Preconstruction ${ }^{137} \mathrm{Cs}$ values dropped immediately following closure of the coffer-cell wall and stayed low during the early fall of 1991 regardless of construction activities. No increase in contaminant resuspension and transport into the Clinch River was observed in association with sheet pile driving or rock armoring operations. However, during periods of no construction activity, there were seasonal increases in ${ }^{137} \mathrm{Cs}$ concentrations at the WOC mouth.

Levels of ${ }^{137} \mathrm{Cs}$ at both stations rose with increasing late fall rainstorms, runoff, and increasing TSS levels as a function of seasonal changes in the WOC watershed. Values remained below the counting error in the absence of concentrations of high suspended solids associated with storms throughout October, November, December, January, and February. Data from the K-1513 station over the same time period showed little or no detectable ${ }^{137} \mathrm{Cs}$ contamination at the K-25 Site water intake.

Increases in ${ }^{137} \mathrm{Cs}$ activity associated with jet grouting were observed at the WOC mouth, though these impacts did not extend downstream to the K-1513 station. Review of radiological data from composite samples collected during concrete cap construction does not indicate increased ${ }^{137} \mathrm{Cs}$ levels associated with these activities.

Cesium-137 activity for both 24-h composite samples (WOC and K-1513) and surface water grab samples increased in conjunction with sediment dredging and rock anchor drilling. At the WOC station, ${ }^{137} \mathrm{Cs}$ values for dredging at WOC were elevated by one to two orders of magnitude relative to nonconstruction reference levels observed from October through February. Values for $\mathrm{K}-1513$ were also elevated for the time frame, though they were one to two orders of magnitude lower than those upstream.

Levels of particle-associated ${ }^{137} \mathrm{Cs}$ at both monitoring stations dropped below detection limits following completion of the sediment-retention structure. Following the establishment of surface sediment control, values were comparable with those observed during nonconstruction monitoring. Release of ${ }^{137} \mathrm{Cs}$ to the Clinch River from WOD through WOCE appears to be related to sediment resuspension and transport from WOL during high-flow events. 


\section{REFERENCES}

Blaylock, B. G., C. J. Ford, M. L. Frank, F. O. Hoffman, and L. A. Hook. 1993. White Oak Creek Embayment Site Characterization and Contaminant Screening Analysis, ORNL/ER-81. Martin Marietta Energy Systems, Inc., Oak Ridge, Tennessee.

EPA (U.S. Environmental Protection Agency). 1979. Methods for the Chemical Analysis of Water and Wastes, EPA-600/4-79-020. EPA, Washington, D.C.

EPA (U.S. Environmental Protection Agency). 1980. Prescribed Procedures for Measurement of Radioactivity in Drinking Water, EPA-600/4-80-032. H. L. Krieger, Environmental Monitoring and Support La sratory, and E. A. Whittaker, Environmental Monitoring Systems Laboratory.

Energy Systems (Martin Marietta Energy Systems, Inc.). 1990. Clinch River RCRA Facility Investigation Plan, ES/ER-1/D1. Martin Marietta Energy Systems Environmental Restoration Program, Oak Ridge, Tennessee.

Ford, C. J. 1993a. Compilation of Statements of Work for the WOCM Project. Oak Ridge National Laboratory, Oak Ridge, Tennessee.

Communication WOCE/070891A.SOW. "Statement of Work for Analytical Services to be Provided by ORNL-ACD to ORNL-ESD in Support of the White Oak Creek Monitoring Project." C. J. Ford, Off-Site Environmental Restoration Program, Martin Marietta Energy Systems, Inc., Oak Ridge, Tennessee, 1991.

Communication WOCEl070891B.SOW. "Statement of Work for Sample Collection and Processing Services To Be Provided by ORNL-OEHP-ESP to ORNL-ESD in Support of the White Oak Creek Monitoring Project." C. J. Ford, Off-Site Environmental Restoration Program, Martin Marietta Energy Systems, Inc., Oak Ridge, Tennessee, 1991.

Communication WOCE/081591A.SOW. "Statement of Work for Collection of Surface Water Quality Data and Samples in Conjunction with the Construction of a Coffer-Cell Structure at the Mouth of White Oak Creek Embayment, August 19, 20, 21 1991. C. J. Ford, Off-Site Environmental Restoration Program, Environmental Sciences Division, Oak Ridge National Laboratory, Martin Marietta Energy Systems, Inc., Oak Ridge, Tennessee, 1991.

Communication WOCEl091791A.SOW. "Statement of Work for Collection of Surface Water Quality Data and Samples in Conjunction with Shoreline Rock Armoring Activities at and Immediately Downstream from the Mouth of White Oak Creek Embayment, Beginning September 18, 1991." C. J. Ford, Off-Site Environmental Restoration Program, Environmental Sciences Division, Oak Ridge National Laboratory, Martin Marietta Energy Systems, Inc., Oak Ridge, Tennessee, 1991. 
Communication WOCE1030692A.SAP. "Plan for the Collection and Analysis of Samples from Jet Grout Rinse Waste Waters at White Oak Creek Embayment." C. J. Ford, Clinch River Environmental Restoration Program, Environmental Sciences Division, Oak Ridge National Laboratory, Martin Marietta Energy Systems, Inc., Oak Ridge, Tennessee, 1992.

Communication WOCE1030992A.SOW. "Statement of Work for Collection of Surface Water Quality Data and Samples in Conjunction with Jet Grout Armoring Activities in the White Oak Creek Embayment, March 1992." C. J. Ford, Clinch River Environmental Restoration Program, Environmental Sciences Division, Oak Ridge National Laboratory, Martin Marietta Energy Systems, Inc., Oak Ridge, Tennessee, 1992.

Communication WOCE1032592A.SOW. "Statement of Work for Collection of Surface Water Quality Data and Samples in Conjunction with Dredging of Coffer Cell Surface Sediments in the White Oak Creek Embayment, March 1992. C. J. Ford, Clinch River Environmental Restoration Program, Environmental Sciences Division, Oak Ridge National Laboratory, Martin Marietta Energy Systems, Inc., Oak Ridge, Tennessee, 1992.

Ford, C. J. 1993b. Compilation of Sample Identification Numbers for the WOCM Project. Martin Marietta Energy Systems, Inc., Oak Ridge, Tennessee.

Kimbrough, C. W., L. W. Long, and L. W. McMahon (eds.). 1990. Environmental Surveillance Procedures Quality Control Program, ESH/Sub/87-21706/1. Martin Marietta Energy Systems, Inc., Oak Ridge, Tennessee.

Kimmel, B. L., and C. J. Ford. 1991. White Oak Creek Embayment, Time-Critical CERCLA Removal Action Water Quality Monitoring Plan. Off-Site Environmental Restoration Program, Martin Marietta Energy Systems, Inc., Oak Ridge, Tennessee.

Kornegay, F. C., D. C. West, S. T. Goodpasture, C. W. Kimbrough, M. F. Tardiff, V. A. Jacobs, and A. R. Wilson. 1990. Oak Ridge Reservation Environmental Report for 1989, ES/ESH-13. Martin Marietta Energy Systems, Inc., Oak Ridge, Tennesse 6

Kornegay, F. C., D. C. West, R. A. Evans, S. T. Goodpasture, M. F. Tardiff, and A. R. Wilson. 1991. Oak Ridge Reservation Environmental Report for 1990, ES/ESH-18. Martin Marietta Energy Systems, Inc., Oak Ridge, Tennessee.

Kornegay, F. C., D. C. West, R. A. Evans, M. F. Tardiff, F. D. Adams, and P. C. Mucke. 1992. Oak Ridge Reservation Environmental Report for 1991, ES/ESH-22. Martin Marietta Energy Systems, Inc., Oak Ridge, Tennessee.

Leslie, M., and B. J. Kimmel. 1992. White Oak Creek Embayment Time-Critical CERCLA Removal Action Regulatory Compliance Study, ORNL/ER/Sub/91-KA931/3. Martin Marietta Energy Systems, Inc., Oak Ridge, Tennessee

Olsen, C. R., I. L. Larsen, P. D. Lowry, C. R. Moriones, C. J. Ford, K. C. Dearstone, R. R. Turner, B. L. Kimmel, and C. C. Brandt. 1992. Transport and Accumulation of Cesium-137 and Mercury in the Clinch River and Watts Bar Reservoir System, ORNL/ER-7. Martin Marietta Energy Systems, Inc., Oak Ridge, Tennessee. 


\section{Appendix A}

\section{PHYSICAL-CHEMICAL MEASUREMENTS AND}

CONTAMINANT ANALYTICAL RESULTS FOR 24-h COMPOSITE SAMPLES FROM THE K-1513 AND WHITE OAK CREEK STATIONS 
A-3

Table A.1. Total ${ }^{137} \mathrm{Cs}$ results from 24 h composite samples

\begin{tabular}{|c|c|c|c|c|}
\hline Date & $\begin{array}{r}\text { River } \\
\text { mile }\end{array}$ & Result & $\begin{array}{l}\text { Rad. } \\
\text { error }\end{array}$ & Units \\
\hline 18 Sep 91 & 20.8 & -1.89 & 17.03 & $\mathrm{pCi} / \mathrm{L}$ \\
\hline 19 Sep 91 & 14.5 & -0.54 & 12.70 & $\mathrm{pCi} / \mathrm{L}$ \\
\hline 19 Sep 91 & 20.8 & 1.08 & 12.70 & $\mathrm{pCi} / \mathrm{L}$ \\
\hline 20 Sep 91 & 20.8 & 4.32 & 12.70 & $\mathrm{pCi} / \mathrm{L}$ \\
\hline 23 Sep 91 & 20.8 & 2.16 & 14.60 & $\mathrm{pCi} / \mathrm{L}$ \\
\hline $24 \operatorname{Sep} 91$ & 14.5 & 3.78 & 4.32 & $\mathrm{pCi} / \mathrm{L}$ \\
\hline $24 \operatorname{Sep} 91$ & 20.8 & -0.54 & 11.89 & $\mathrm{pCi} / \mathrm{L}$ \\
\hline $25 \operatorname{Sep} 91$ & 14.5 & -0.54 & 10.00 & $\mathrm{pCi} / \mathrm{L}$ \\
\hline 25 Sep 91 & 20.8 & 5.14 & 9.73 & $\mathrm{pCi} / \mathrm{L}$ \\
\hline $26 \operatorname{Sep} 91$ & 14.5 & -1.08 & 11.08 & $\mathrm{pCi} / \mathrm{L}$ \\
\hline 26 Sep 91 & 20.8 & 10.00 & 7.03 & $\mathrm{pCi} / \mathrm{L}$ \\
\hline 27 Sep 91 & 14.5 & -2.70 & 14.87 & $\mathrm{pCi} / \mathrm{L}$ \\
\hline 27 Sep 91 & 20.8 & 12.70 & 14.06 & $\mathrm{pCi} / \mathrm{L}$ \\
\hline 30 Sep 91 & 14.5 & -3.51 & 15.95 & $\mathrm{pCi} / \mathrm{L}$ \\
\hline $30 \operatorname{Sep} 91$ & 20.8 & -2.16 & 17.57 & $\mathrm{pCi} / \mathrm{L}$ \\
\hline 01 Oct 91 & 14.5 & -1.08 & 13.24 & $\mathrm{pCi} / \mathrm{L}$ \\
\hline 01 Oct 91 & 20.8 & 2.16 & 10.81 & $\mathrm{pCi} / \mathrm{L}$ \\
\hline 02 Oct 91 & 14.5 & -2.16 & 9.73 & $\mathrm{pCi} / \mathrm{L}$ \\
\hline 02 Oct 91 & 20.8 & 1.08 & 14.60 & $\mathrm{pCi} / \mathrm{L}$ \\
\hline 03 Oct 91 & 14.5 & 1.08 & 9.73 & $\mathrm{pCi} / \mathrm{L}$ \\
\hline 03 Oct 91 & 20.8 & 1.89 & 11.89 & $\mathrm{pCi} / \mathrm{L}$ \\
\hline 04 Oct 91 & 14.5 & -1.08 & 8.11 & $\mathrm{pCi} / \mathrm{L}$ \\
\hline 04 Oct 91 & 20.8 & 1.35 & 11.89 & $\mathrm{pCi} / \mathrm{L}$ \\
\hline 07 Oct 91 & 14.5 & 5.14 & 4.05 & $\mathrm{pCi} / \mathrm{L}$ \\
\hline 07 Oct 91 & 20.8 & 0.54 & 6.22 & $\mathrm{pCi} / \mathrm{L}$ \\
\hline 29 Oct 91 & 14.5 & 1.62 & 7.84 & $\mathrm{pCi} / \mathrm{L}$ \\
\hline 29 Oct 91 & 20.8 & 1.89 & 14.06 & $\mathrm{pCi} / \mathrm{L}$ \\
\hline 30 Oct 91 & 20.8 & 11.62 & 8.92 & $\mathrm{pCi} / \mathrm{L}$ \\
\hline 31 Oct 91 & 14.5 & -1.62 & 10.00 & $\mathrm{pCi} / \mathrm{L}$ \\
\hline 31 Oct 91 & 20.8 & -1.62 & 10.00 & $\mathrm{pCi} / \mathrm{L}$ \\
\hline 01 Nov 91 & 20.8 & 0.54 & 10.81 & $\mathrm{pCi} / \mathrm{L}$ \\
\hline
\end{tabular}


Table A.1 (continued)

\begin{tabular}{|c|c|c|c|c|}
\hline Date & $\begin{array}{r}\text { River } \\
\text { mile }\end{array}$ & Result & $\begin{array}{l}\text { Rad. } \\
\text { error }\end{array}$ & Units \\
\hline 04 Nov 91 & 20.8 & -3.51 & 12.70 & $\mathrm{pCi} / \mathrm{L}$ \\
\hline 05 Nov 91 & 14.5 & -4.32 & 11.89 & $\mathrm{pCi} / \mathrm{L}$ \\
\hline 05 Nov 91 & 20.8 & 3.51 & 17.30 & pCi/L \\
\hline 06 Nov 91 & 14.5 & 12.97 & 8.92 & $\mathrm{pCi} / \mathrm{L}$ \\
\hline 06 Nov 91 & 20.8 & 11.89 & 11.89 & $\mathrm{pCi} / \mathrm{L}$ \\
\hline 06 Nov 91 & 20.8 & 2.70 & 11.89 & $\mathrm{pCi} / \mathrm{L}$ \\
\hline 07 Nov 91 & 20.8 & -3.51 & 18.38 & $\mathrm{pCi} i$ \\
\hline 11 Nov 91 & 20.8 & -1.62 & 13.79 & $\mathrm{pCi} / \mathrm{L}$ \\
\hline 15 Nov 91 & 20.8 & 6.49 & 6.22 & $\mathrm{pCi} / \mathrm{L}$ \\
\hline 18 Nov 91 & 20.8 & -0.81 & 13.79 & $\mathrm{pCi} / \mathrm{L}$ \\
\hline 19 Nov 91 & 14.5 & -0.81 & 15.41 & $\mathrm{pCi} / \mathrm{L}$ \\
\hline 19 Nov 91 & 20.8 & -0.54 & 8.92 & $\mathrm{pCi} / \mathrm{L}$ \\
\hline 20 Nov 91 & 20.8 & 1.62 & 11.89 & $\mathrm{pCi} / \mathrm{L}$ \\
\hline 21 Nov 91 & 14.5 & 0.81 & 8.38 & $\mathrm{pCi} / \mathrm{L}$ \\
\hline 22 Nov 91 & 20.8 & 40.55 & 10.81 & $\mathrm{pCi} / \mathrm{L}$ \\
\hline 25 Nov 91 & 20.8 & 0.81 & 13.79 & $\mathrm{pCi} / \mathrm{L}$ \\
\hline 26 Nov 91 & 14.5 & 1.35 & 16.22 & pCi/L \\
\hline 02 Dec 91 & 14.5 & 1.89 & 17.30 & $\mathrm{pCi} / \mathrm{L}$ \\
\hline $02 \operatorname{Dec} 91$ & 20.8 & 54.06 & 10.81 & $\mathrm{pCi} / \mathrm{L}$ \\
\hline $03 \operatorname{Dec} 91$ & 14.5 & -7.30 & 15.41 & $\mathrm{pCi} / \mathrm{L}$ \\
\hline 03 Dec 91 & 20.8 & 62.17 & 13.52 & pCi/L. \\
\hline $04 \operatorname{Dec} 91$ & 14.5 & -3.78 & 14.87 & $\mathrm{pCi} / \mathrm{L}$ \\
\hline $04 \operatorname{Dec} 91$ & 20.8 & 9.73 & 19.19 & $\mathrm{pCi} / \mathrm{L}$ \\
\hline $05 \operatorname{Dec} 91$ & 14.5 & 13.79 & 18.92 & $\mathrm{PCi} / \mathrm{L}$ \\
\hline 05 Dec 91 & 20.8 & -6.76 & 19.19 & $\mathrm{pCi} / \mathrm{L}$ \\
\hline $06 \operatorname{Dec} 91$ & 14.5 & 0.54 & 8.92 & $\mathrm{pCi} / \mathrm{L}$ \\
\hline $06 \operatorname{Dec} 91$ & 20.8 & 2.97 & 15.14 & $\mathrm{pCi} / \mathrm{L}$ \\
\hline $09 \operatorname{Dec} 91$ & 14.5 & 4.60 & 10.54 & $\mathrm{pCi} / \mathrm{L}$ \\
\hline $10 \operatorname{Dec} 91$ & 14.5 & 2.70 & 16.22 & $\mathrm{pCi} / \mathrm{L}$ \\
\hline $12 \operatorname{Dec} 91$ & 14.5 & 2.70 & 20.00 & $\mathrm{pCi} / \mathrm{L}$ \\
\hline $13 \operatorname{Dec} 91$ & 14.5 & 7.57 & 19.19 & $\mathrm{pCi} / \mathrm{L}$ \\
\hline
\end{tabular}




\section{A-5}

Table A.1 (continued)

\begin{tabular}{|c|c|c|c|c|}
\hline Date & $\begin{array}{c}\text { River } \\
\text { mile }\end{array}$ & Result & $\begin{array}{l}\text { Rad. } \\
\text { error }\end{array}$ & Units \\
\hline 16 Dec 91 & 14.5 & 2.16 & 8.92 & $\mathrm{pCi} / \mathrm{L}$ \\
\hline $17 \operatorname{Dec} 91$ & 14.5 & -4.05 & 13.24 & $\mathrm{pCi} / \mathrm{L}$ \\
\hline $17 \operatorname{Dec} 91$ & 20.8 & 5.14 & 10.27 & $\mathrm{pCi} / \mathrm{L}$ \\
\hline $18 \operatorname{Dec} 91$ & 14.5 & 2.16 & 10.00 & $\mathrm{pCi} / \mathrm{L}$ \\
\hline $19 \operatorname{Dec} 91$ & 14.5 & -0.54 & 8.92 & $\mathrm{pCi} / \mathrm{L}$ \\
\hline $20 \operatorname{Dec} 91$ & 14.5 & 6.76 & 7.84 & $\mathrm{pCi} / \mathrm{L}$ \\
\hline 26 Dec 91 & 14.5 & -2.43 & 18.38 & pCilL \\
\hline 26 Dec 91 & 20.8 & 3.24 & 4.32 & $\mathrm{pCi} / \mathrm{L}$ \\
\hline $30 \operatorname{Dec} 91$ & 14.5 & 4.87 & 12.43 & $\mathrm{pCi} / \mathrm{L}$ \\
\hline 30 Dec 91 & 20.8 & 1.08 & 15.14 & $\mathrm{pCi} / \mathrm{L}$ \\
\hline 31 Dec 91 & 14.5 & 4.87 & 7.84 & $\mathrm{pCi} / \mathrm{L}$ \\
\hline $02 \operatorname{Jan} 92$ & 14.5 & 1.08 & 7.84 & $\mathrm{pCi} / \mathrm{L}$ \\
\hline 03 Jan 92 & 14.5 & -5.95 & 14.06 & $\mathrm{pCi} / \mathrm{L}$ \\
\hline $06 \operatorname{Jan} 92$ & 14.5 & 0.81 & 14.87 & $\mathrm{pCi} / \mathrm{L}$ \\
\hline $06 \operatorname{Jan} 92$ & 20.8 & 10.27 & 16.49 & $\mathrm{pCi} / \mathrm{L}$ \\
\hline $07 \operatorname{Jan} 92$ & 14.5 & 6.76 & 9.73 & pCi/L \\
\hline $09 \operatorname{Jan} 92$ & 14.5 & 2.43 & 10.81 & $\mathrm{p} C \mathrm{Ci} / \mathrm{L}$ \\
\hline $13 \operatorname{Jan} 92$ & 14.5 & 10.54 & 9.19 & pCi/L \\
\hline $14 \operatorname{Jan} 9 ?$ & 14.5 & -0.81 & 12.16 & $\mathrm{pCi} / \mathrm{L}$ \\
\hline $15 \operatorname{Jan} 92$ & 14.5 & -2.43 & 25.14 & $\mathrm{pCi} / \mathrm{L}$ \\
\hline $16 \operatorname{Jan} 92$ & 14.5 & 3.51 & 12.43 & $\mathrm{pCi} / \mathrm{L}$ \\
\hline $17 \operatorname{Jan} 92$ & 14.5 & -1.89 & 14.06 & $\mathrm{pCi} / \mathrm{L}$ \\
\hline $20 \operatorname{Jan} 92$ & 14.5 & 3.78 & 10.00 & $\mathrm{pCi} / \mathrm{L}$ \\
\hline $21 \operatorname{Jan} 92$ & 14.5 & -4.32 & 12.70 & $\mathrm{pCi} / \mathrm{L}$ \\
\hline $22 \operatorname{Jan} 92$ & 14.5 & -8.92 & 19.73 & $\mathrm{pCi} / \mathrm{L}$ \\
\hline $23 \operatorname{Jan} 92$ & 14.5 & 5.95 & 10.54 & $\mathrm{pCi} / \mathrm{L}$ \\
\hline $24 \operatorname{Jan} 92$ & 14.5 & 2.16 & 6.22 & $\mathrm{pCi} / \mathrm{L}$ \\
\hline $27 \operatorname{Jan} 92$ & 14.5 & 5.95 & 4.32 & $\mathrm{pCi} / \mathrm{L}$ \\
\hline $28 \operatorname{Jan} 92$ & 14.5 & 1.62 & 8.65 & $\mathrm{pCi} / \mathrm{L}$ \\
\hline $29 \operatorname{Jan} 92$ & 14.5 & -2.70 & 11.89 & $\mathrm{pCi} / \mathrm{L}$ \\
\hline $30 \mathrm{Jan} 92$ & 14.5 & -10.81 & 22.43 & $\mathrm{pCi} / \mathrm{L}$ \\
\hline
\end{tabular}


A-6

Table A.1 (continued)

\begin{tabular}{|c|c|c|c|c|}
\hline Date & $\begin{array}{r}\text { River } \\
\text { mile }\end{array}$ & Result & $\begin{array}{l}\text { Rad. } \\
\text { error }\end{array}$ & Units \\
\hline 05 Feb 92 & 14.5 & 5.14 & 7.03 & $\mathrm{pCi} / \mathrm{L}$ \\
\hline 05 Feb 92 & 14.5 & -1.62 & 12.97 & $\mathrm{pCi} / \mathrm{L}$ \\
\hline 05 Feb 92 & 20.8 & -0.81 & 15.68 & $\mathrm{pCi} / \mathrm{L}$ \\
\hline 06 Feb 92 & 14.5 & 6.49 & 11.08 & $\mathrm{pCi} / \mathrm{L}$ \\
\hline 06 Feb 92 & 20.8 & -1.35 & 14.60 & $\mathrm{pCi} / \mathrm{L}$ \\
\hline 07 Feb 92 & 14.5 & 2.70 & 7.84 & $\mathrm{pCi} / \mathrm{L}$ \\
\hline 07 Feb 92 & 20.8 & -11.08 & 22.98 & $\mathrm{pCi} / \mathrm{L}$ \\
\hline 11 Feb 92 & 14.5 & 4.87 & 8.92 & $\mathrm{pCi} / \mathrm{L}$ \\
\hline 12 Feb 92 & 14.5 & -1.62 & 10.00 & $\mathrm{pCi} / \mathrm{L}$ \\
\hline 12 Feb 92 & 20.8 & 19.19 & 13.24 & $\mathrm{pCi} / \mathrm{L}$ \\
\hline 13 Feb 92 & 14.5 & -0.54 & 12.97 & $\mathrm{pCi} / \mathrm{L}$ \\
\hline 14 Feb 92 & 14.5 & -2.16 & 21.62 & $\mathrm{pCi} / \mathrm{L}$ \\
\hline 18 Feb 92 & 14.5 & -0.27 & 26.22 & $\mathrm{pCi} / \mathrm{L}$ \\
\hline 18 Feb 92 & 20.8 & 14.87 & 8.65 & $\mathrm{pCi} / \mathrm{L}$ \\
\hline 19 Feb 92 & 14.5 & 3.78 & 8.92 & $\mathrm{pCi} / \mathrm{L}$ \\
\hline 19 Feb 92 & 20.8 & 21.08 & 11.35 & $\mathrm{pCi} / \mathrm{L}$ \\
\hline 21 Feb 92 & 14.5 & 0.81 & 15.14 & $\mathrm{pCi} / \mathrm{L}$ \\
\hline 21 Feb 92 & 20.8 & 29.73 & 13.52 & $\mathrm{pCi} / \mathrm{L}$ \\
\hline 25 Feb 92 & 14.5 & -3.24 & 12.70 & $\mathrm{pCi} / \mathrm{L}$ \\
\hline 25 Feb 92 & 20.8 & 35.14 & 10.81 & $\mathrm{pCi} / \mathrm{L}$ \\
\hline 26 Feb 92 & 20.8 & 37.84 & 13.52 & $\mathrm{pCi} / \mathrm{L}$ \\
\hline 27 Feb 92 & 14.5 & -2.43 & 15.41 & $\mathrm{pCi} / \mathrm{L}$ \\
\hline 27 Feb 92 & 20.8 & 54.06 & 13.52 & $\mathrm{pCi} / \mathrm{L}$ \\
\hline 28 Feb 92 & 14.5 & 7.84 & 11.89 & $\mathrm{pCi} / \mathrm{L}$ \\
\hline $03 \operatorname{Mar} 92$ & 14.5 & 1.35 & 13.24 & $\mathrm{pCi} / \mathrm{L}$ \\
\hline 03 Mar 92 & 20.8 & 37.84 & 10.81 & $\mathrm{pCi} / \mathrm{L}$ \\
\hline 05 Mar 92 & 14.5 & -7.57 & 19.46 & $\mathrm{pCi} / \mathrm{L}$ \\
\hline $05 \operatorname{Mar} 92$ & 20.8 & 29.73 & 10.81 & $\mathrm{pCi} / \mathrm{L}$ \\
\hline 06 Mar 92 & 14.5 & 2.16 & 13.24 & $\mathrm{pCi} / \mathrm{L}$ \\
\hline 06 Mar 92 & 20.8 & 19.73 & 13.24 & $\mathrm{pCi} / \mathrm{L}$ \\
\hline 10 Mar 92 & 14.5 & 7.30 & 7.57 & $\mathrm{pCi} / \mathrm{L}$ \\
\hline
\end{tabular}


Table A.1 (continued)

\begin{tabular}{|c|c|c|c|c|}
\hline Date & $\begin{array}{r}\text { River } \\
\text { mile }\end{array}$ & Result & $\begin{array}{l}\text { Rad. } \\
\text { error }\end{array}$ & Units \\
\hline $10 \operatorname{Mar} 92$ & 20.8 & 32.44 & 16.22 & $\mathrm{pCi} / \mathrm{L}$ \\
\hline 11 Mar 92 & 14.5 & 1.08 & 6.22 & $\mathrm{pCi} / \mathrm{L}$ \\
\hline $12 \operatorname{Mar} 92$ & 14.5 & 0.27 & 6.76 & $\mathrm{pCi} / \mathrm{L}$ \\
\hline 13 Mar 92 & 14.5 & 4.32 & 15.14 & $\mathrm{pCi} / \mathrm{L}$ \\
\hline $17 \operatorname{Mar} 92$ & 14.5 & -0.54 & 9.73 & $\mathrm{pCi} / \mathrm{L}$ \\
\hline $18 \operatorname{Mar} 92$ & 14.5 & 2.16 & 13.24 & $\mathrm{pCi} / \mathrm{L}$ \\
\hline $18 \operatorname{Mar} 92$ & 20.8 & 29.73 & 13.52 & $\mathrm{pCi} / \mathrm{L}$ \\
\hline $19 \operatorname{Mar} 92$ & 14.5 & -5.41 & 29.73 & $\mathrm{pCi} / \mathrm{L}$ \\
\hline $19 \operatorname{Mar} 92$ & 20.8 & 51.36 & 16.22 & $\mathrm{pCi} / \mathrm{L}$ \\
\hline $20 \operatorname{Mar} 92$ & 14.5 & 3.24 & 12.97 & $\mathrm{pCi} / \mathrm{L}$ \\
\hline 20 Mar 92 & 20.8 & 59.47 & 13.52 & pCill \\
\hline $24 \operatorname{Mar} 92$ & 14.5 & -1.62 & 14.06 & $\mathrm{pCi} / \mathrm{L}$ \\
\hline $24 \operatorname{Mar} 92$ & 20.8 & 37.84 & 13.52 & $\mathrm{pCi} / \mathrm{L}$ \\
\hline $25 \operatorname{Mar} 92$ & 14.5 & 0.54 & 8.11 & $\mathrm{pCi} / \mathrm{L}$ \\
\hline 25 Mar 92 & 20.8 & 22.16 & 9.73 & $\mathrm{pCi} / \mathrm{L}$ \\
\hline $26 \operatorname{Mar} 92$ & 14.5 & 3.51 & 7.03 & $\mathrm{pCi} / \mathrm{L}$ \\
\hline $26 \operatorname{Mar} 92$ & 20.8 & 64.87 & 24.33 & $\mathrm{pCi} / \mathrm{L}$ \\
\hline 27 Mar 92 & 14.5 & 10.81 & 10.00 & $\mathrm{pCi} / \mathrm{L}$ \\
\hline $27 \operatorname{Mar} 92$ & 20.8 & 170.29 & 29.73 & $\mathrm{pCi} / \mathrm{L}$ \\
\hline 31 Mar 92 & 14.5 & 1.89 & 14.06 & $\mathrm{pCi} / \mathrm{L}$ \\
\hline 31 Mar 92 & 20.8 & 19.46 & 11.89 & $\mathrm{pCi} / \mathrm{L}$ \\
\hline 01 Apr 92 & 14.5 & 6.22 & 14.06 & $\mathrm{pCi} / \mathrm{L}$ \\
\hline 01 Apr 92 & 20.8 & 2.16 & 10.81 & $\mathrm{pCi} / \mathrm{L}$ \\
\hline 02 Apr 92 & 14.5 & 7.84 & 11.35 & $\mathrm{pCi} / \mathrm{L}$ \\
\hline 02 Apr 92 & 20.8 & 62.17 & 13.52 & $\mathrm{pCi} / \mathrm{L}$ \\
\hline 03 Apr 92 & 14.5 & 7.57 & 8.92 & $\mathrm{pCi} / \mathrm{L}$ \\
\hline 03 Apr 92 & 20.8 & 7.84 & 6.49 & $\mathrm{pCi} / \mathrm{L}$ \\
\hline 07 Apr 92 & 14.5 & -6.22 & 18.11 & $\mathrm{pCi} / \mathrm{L}$ \\
\hline 07 Apr 92 & 14.5 & -6.22 & 18.11 & $\mathrm{pCi} / \mathrm{L}$ \\
\hline 07 Apr 92 & 20.8 & 25.41 & 14.60 & $\mathrm{pCi} / \mathrm{L}$ \\
\hline 07 Apr 92 & 20.8 & 25.41 & 14.60 & $\mathrm{pCi} / \mathrm{L}$ \\
\hline
\end{tabular}


A.8

Table A.1 (continued)

\begin{tabular}{|c|c|c|c|c|}
\hline Date & $\begin{array}{r}\text { River } \\
\text { mile }\end{array}$ & Result & $\begin{array}{l}\text { Rad. } \\
\text { error }\end{array}$ & Units \\
\hline 08 Apr 92 & 14.5 & 7.03 & 14.06 & $\mathrm{pCi} / \mathrm{L}$ \\
\hline 08 Apr 92 & 20.8 & 37.84 & 10.81 & $\mathrm{pCi} / \mathrm{L}$ \\
\hline 09 Арг 92 & 14.5 & -1.62 & 15.41 & $\mathrm{pCi} / \mathrm{L}$ \\
\hline 09 Apr 92 & 20.8 & 35.14 & 13.52 & $\mathrm{pCi} / \mathrm{L}$ \\
\hline 10 Apr 92 & 14.5 & 5.95 & 18.11 & $\mathrm{pCi} / \mathrm{L}$ \\
\hline 14 Apr 92 & 14.5 & 5.14 & 16.22 & $\mathrm{pCi} / \mathrm{L}$ \\
\hline 15 Apr 92 & 14.5 & 5.95 & 5.41 & $\mathrm{pCi} / \mathrm{L}$ \\
\hline 15 Apr 92 & 20.8 & 59.47 & 16.22 & $\mathrm{pCi} / \mathrm{L}$ \\
\hline 16 Apr 92 & 14.5 & -1.35 & 9.19 & $\mathrm{pCi} / \mathrm{L}$ \\
\hline 16 Apr 92 & 20.8 & 37.84 & 16.22 & $\mathrm{pCi} / \mathrm{L}$ \\
\hline 22 Apr 92 & 14.5 & -0.54 & 16.76 & $\mathrm{pCi} / \mathrm{L}$ \\
\hline 22 Apr 92 & 20.8 & 4.87 & 6.76 & $\mathrm{pCi} / \mathrm{L}$ \\
\hline 29 Apr 92 & 14.5 & 2.43 & 7.03 & $\mathrm{pCi} / \mathrm{L}$ \\
\hline 29 Apr 92 & 20.8 & 0.54 & 12.97 & $\mathrm{pCi} / \mathrm{L}$ \\
\hline 06 May 92 & 14.5 & 2.97 & 7.03 & $\mathrm{pCi} / \mathrm{L}$ \\
\hline D6 May 92 & 20.8 & -1.35 & 13.79 & $\mathrm{pCi} / \mathrm{L}$ \\
\hline 13 May 92 & 14.5 & 4.87 & 16.22 & $\mathrm{pCi} / \mathrm{L}$ \\
\hline 13 May 92 & 20.8 & 7.57 & 11.89 & $\mathrm{pCi} / \mathrm{L}$ \\
\hline 20 May 92 & 14.5 & 1.89 & 18.11 & $\mathrm{pCi} / \mathrm{L}$ \\
\hline 20 May 92 & 20.8 & 2.43 & 8.11 & $\mathrm{pCi} / \mathrm{L}$ \\
\hline 27 May 92 & 14.5 & -0.81 & 17.30 & $\mathrm{pCi} / \mathrm{L}$ \\
\hline 27 May 92 & 20.8 & 4.87 & 14.06 & $\mathrm{pCi} / \mathrm{L}$ \\
\hline 04 Jun 92 & 14.5 & -3.24 & 11.62 & $\mathrm{pCi} / \mathrm{L}$ \\
\hline 04 Jun 92 & 20.8 & 6.49 & 9.73 & $\mathrm{pCi} / \mathrm{L}$ \\
\hline 10 Jun 92 & 14.5 & -0.27 & 3.24 & $\mathrm{pCi} / \mathrm{L}$ \\
\hline 10 Jun 92 & 20.8 & 0.54 & 4.05 & $\mathrm{pCi} / \mathrm{L}$ \\
\hline 17 Jun 92 & 14.5 & 7.30 & 14.33 & $\mathrm{pCi} / \mathrm{L}$ \\
\hline 17 Jun 92 & 20.8 & 18.65 & 13.24 & $\mathrm{pCi} / \mathrm{L}$ \\
\hline 24 Jun 92 & 14.5 & 1.62 & 12.16 & $\mathrm{pCi} / \mathrm{L}$ \\
\hline 24 Jun 92 & 20.8 & -1.89 & 24.60 & $\mathrm{pCi} / \mathrm{L}$ \\
\hline 30 Jun 92 & 14.5 & -5.95 & 18.38 & $\mathrm{pCi} / \mathrm{L}$ \\
\hline
\end{tabular}


A-9

Table A.1 (continued)

\begin{tabular}{ccccc}
\hline \multicolumn{1}{c}{ Date } & $\begin{array}{c}\text { River } \\
\text { mile }\end{array}$ & \multicolumn{1}{c}{ Result } & \multicolumn{1}{c}{ Rad. } \\
\hline 30 Jun 92 & 20.8 & 1.35 & 7.03 & UCi/L \\
08 Jul 92 & 14.5 & 7.84 & 3.51 & $\mathrm{pCi} / \mathrm{L}$ \\
08 Jul 92 & 20.8 & 26.22 & 7.57 & $\mathrm{pCi} / \mathrm{L}$ \\
15 Jul 92 & 14.5 & 1.35 & 9.19 & $\mathrm{pCi} / \mathrm{L}$ \\
15 Jul 92 & 20.8 & 25.14 & 13.79 & $\mathrm{pCi} / \mathrm{L}$ \\
23 Jul 92 & 14.5 & 10.812 & 8.92 & $\mathrm{pCi} / \mathrm{L}$ \\
23 Jul 92 & 20.8 & 0.27 & 15.14 & $\mathrm{pCi} / \mathrm{L}$ \\
29 Jul 92 & 20.8 & 5.14 & 8.92 & $\mathrm{pCi} / \mathrm{L}$ \\
\hline
\end{tabular}


A-10

Table A.2 Water chemistry results from 24-h composite samples

\begin{tabular}{|c|c|c|c|c|}
\hline Date & $\begin{array}{c}\text { River } \\
\text { mile } \\
\end{array}$ & Analyte & Result & Units \\
\hline $18 \operatorname{Sep} 91$ & 20.8 & Turbidity & 1.5 & NTU \\
\hline $18 \operatorname{Sep} 91$ & 20.8 & Tot. susp. solids & 5.0 & $\mathrm{mg} / \mathrm{L}$ \\
\hline 19 Sep 91 & 14.5 & Turbidity & 1.7 & NTU \\
\hline 19 Sep 91 & 14.5 & Tot. susp. solids & 11.0 & $\mathrm{mg} / \mathrm{L}$ \\
\hline 19 Sep 91 & 20 & Turbidity & 1.1 & NTU \\
\hline $20 \operatorname{Sep} 91$ & 20.8 & Turbidity & 1.9 & NTU \\
\hline 20 Sep 91 & 20.8 & Tot. susp. solids & 5.0 & $\mathrm{mg} / \mathrm{L}$ \\
\hline 23 Sep 91 & 20.8 & Turbidity & 1.2 & NTU \\
\hline 23 Sep 91 & 20.8 & Tot. susp. solids & 5.0 & $\mathrm{mg} / \mathrm{L}$ \\
\hline 24 Sep 91 & 14.5 & Tot. susp. solids & 5.0 & $\mathrm{mg} / \mathrm{L}$ \\
\hline $24 \operatorname{Sep} 91$ & 14.5 & Turbidity & 1.8 & NTU \\
\hline 24 Sep 91 & 20.8 & Tot. susp. solids & 5.0 & $\mathrm{mg} / \mathrm{L}$ \\
\hline 24 Sep 91 & 20.8 & Turbidity & 1.8 & NTU \\
\hline $25 \operatorname{Sep} 91$ & 14.5 & Turbidity & 1.4 & NTU \\
\hline 25 Sep 91 & 14.5 & Tot. susp. solids & 5.0 & $\mathrm{mg} / \mathrm{L}$ \\
\hline 25 Sep 91 & 20.8 & Tot. susp. solids & 5.0 & $\mathrm{mg} / \mathrm{L}$ \\
\hline 25 Sep 91 & 20.8 & Turbidity & 2.2 & NTU \\
\hline $26 \operatorname{Sep} 91$ & 14.5 & Tot. susp. solids & 5.0 & $\mathbf{m g} / \mathbf{L}$ \\
\hline $26 \operatorname{Sep} 91$ & 14.5 & Turbidity & 1.5 & NTU \\
\hline $26 \operatorname{Sep} 91$ & 20.8 & Turbidity & 2.3 & NTU \\
\hline $26 \operatorname{Sep} 91$ & 20.8 & Tot. susp. solids & 5.0 & $\mathrm{mg} / \mathrm{L}$ \\
\hline $27 \operatorname{Sep} 91$ & 14.5 & Turbidity & 1.9 & NTU \\
\hline 27 Sep 91 & 14.5 & Tot. susp. solids & 5.0 & $\mathrm{mg} / \mathrm{L}$ \\
\hline 27 Sep 91 & 20.8 & Tot. susp. solids & 5.0 & $\mathrm{mg} / \mathrm{L}$ \\
\hline 27 Sep 91 & 20.8 & Turbidity & 2.4 & NTU \\
\hline 30 Sep 91 & 14.5 & Turbidity & 1.1 & NTU \\
\hline 30 Sep 91 & 14.5 & Tot. susp. solids & 5.0 & $\mathrm{mg} / \mathrm{L}$ \\
\hline 30 Sep 91 & 20.8 & Turbidity & 1.5 & NTU \\
\hline 30 Sep 91 & 20.8 & Tot. susp. solids & 5.0 & $\mathrm{mg} / \mathrm{L}$ \\
\hline 01 Oct 91 & 14.5 & Tot. susp. solids & 5.0 & $\mathrm{mg} / \mathrm{L}$ \\
\hline 01 Oct 91 & 14.5 & Turbidity & 1.5 & NTU \\
\hline
\end{tabular}


Table A.2 (continued)

\begin{tabular}{|c|c|c|c|c|}
\hline Date & $\begin{array}{l}\text { River } \\
\text { mile }\end{array}$ & Analyte & Result & Units \\
\hline 01 Oct 91 & 20.8 & Turbidity & 1.5 & NTU \\
\hline 01 Oct 91 & 20.8 & Tot. susp. solids & 5.0 & $\mathrm{mg} / \mathrm{L}$ \\
\hline 03 Oct 91 & 14.5 & Turbidity & 1.1 & NTU \\
\hline 03 Oct 91 & 14.5 & Tot. susp. solids & 5.0 & $\mathrm{mg} / \mathrm{L}$ \\
\hline 03 Oct 91 & 20.8 & Turbidity & 1.1 & NTU \\
\hline 03 Oct 91 & 20.8 & Tot. susp. solids & 5.0 & $\mathrm{mg} / \mathrm{L}$ \\
\hline 04 Oct 91 & 14.5 & Tot. susp. solids & 5.0 & $\mathrm{mg} / \mathrm{L}$ \\
\hline 04 Oct 91 & 14.5 & Turbidity & 2.4 & NTU \\
\hline 04 Oct 91 & 20.8 & Turbidity & 1.7 & NTU \\
\hline 04 Oct 91 & 20.8 & Tot. susp. solids & 5.0 & $\mathrm{mg} / \mathrm{L}$ \\
\hline 07 Oct 91 & 14.5 & Turbidity & 0.8 & NTU \\
\hline 07 Oct 91 & 14.5 & Tot. susp. solids & 5.0 & $\mathbf{m g} / \mathbf{L}$ \\
\hline 07 Oct 91 & 20.8 & Turbidity & 1.0 & NTU \\
\hline 07 Oct 91 & 20.8 & Tot. susp. solids & 5.0 & $\mathbf{m g} / \mathbf{L}$ \\
\hline 08 Oct 91 & 14.5 & Tot. susp. solids & 5.0 & $\mathrm{mg} / \mathrm{L}$ \\
\hline 08 Oct 91 & 14.5 & Turbidity & 1.7 & NTU \\
\hline 29 Oct 91 & 14.5 & Tot. susp. solids & 5.0 & $\mathbf{m g} / \mathbf{L}$ \\
\hline 29 Oct 91 & 14.5 & Turbidity & 1.0 & NTU \\
\hline 29 Oct 91 & 20.8 & Tot. susp. solids & 2.0 & $\mathrm{mg} / \mathrm{L}$ \\
\hline 29 Oct 91 & 20.8 & Turbidity & 0.8 & NTU \\
\hline 30 Oct 91 & 20.8 & Tot. susp. solids & 6.0 & $\mathrm{mg} / \mathrm{L}$ \\
\hline 30 Oct 91 & 20.8 & Turbidity & 2.7 & NTU \\
\hline 31 Oct 91 & 14.5 & Tot. susp. solids & 5.0 & $\mathrm{mg} / \mathrm{L}$ \\
\hline 31 Oct 91 & 14.5 & Turbidity & 1.5 & NTU \\
\hline 31 Oct 91 & 20.8 & Tot. susp. solids & 5.0 & $\mathbf{m g} / \mathrm{L}$ \\
\hline 31 Oct 91 & 20.8 & Turbidity & 1.1 & NTU \\
\hline 01 Nov 91 & 20.8 & Turbidity & 1.2 & NTU \\
\hline 01 Nov 91 & 20.8 & Tot. susp. solids & 5.0 & $\mathrm{mg} / \mathrm{L}$ \\
\hline 04 Nov 91 & 20.8 & Turbidity & 1.1 & NTU \\
\hline 04 Nov 91 & 20.8 & Tot. susp. solids & 5.0 & $\mathrm{mg} / \mathrm{L}$ \\
\hline 05 Nov 91 & 14.5 & Tot. susp. solids & 6.0 & $\mathrm{mg} / \mathrm{L}$ \\
\hline
\end{tabular}


Table A.2 (continued)

\begin{tabular}{|c|c|c|c|c|}
\hline Date & $\begin{array}{c}\text { River } \\
\text { mile }\end{array}$ & Analyte & Result & Units \\
\hline 05 Nov 91 & 14.5 & Turbidity & 2.3 & NTU \\
\hline 05 Nov 91 & 20.8 & Tot. susp. solids & 19.0 & $\mathrm{mg} / \mathrm{L}$ \\
\hline 05 Nov 91 & 20.8 & Turbidity & 2.9 & NTU \\
\hline 06 Nov 91 & 14.5 & Tot. susp. solids & 5.0 & $\mathbf{m g} / \mathbf{L}$ \\
\hline 06 Nov 91 & 14.5 & Turbidity & 1.4 & NTU \\
\hline 06 Nov 91 & 20.8 & Tot. susp. solids & 5.0 & $\mathrm{mg} / \mathrm{L}$ \\
\hline 06 Nov 91 & 20.8 & Turbidity & 2.0 & NTU \\
\hline 06 Nov 91 & 20.8 & Turbidity & 3.4 & NTU \\
\hline 06 Nov 91 & 20.8 & Tot. susp. solids & 51.0 & $\mathrm{mg} / \mathrm{L}$ \\
\hline 11 Nov 91 & 20.8 & Turbidity & 1.2 & NTU \\
\hline 11 Nov 91 & 20.8 & Tot. susp. solids & 5.0 & $\mathrm{mg} / \mathrm{L}$ \\
\hline 12 Nov 91 & 14.5 & Tot. susp. solids & 5.0 & $\mathrm{mg} / \mathrm{L}$ \\
\hline 12 Nov 91 & 14.5 & Turbidity & 1.5 & NTU \\
\hline 12 Nov 91 & 20.8 & Turbidity & 1.9 & NTU \\
\hline 12 Nov 91 & 20.8 & Tot. susp. solids & 5.0 & $\mathrm{mg} / \mathrm{L}$ \\
\hline 14 Nov 91 & 14.5 & Turbidity & 1.2 & NTU \\
\hline 14 Nov 91 & 14.5 & Tot. susp. solids & 5.0 & $\mathrm{mg} / \mathrm{L}$ \\
\hline 14 Nov 91 & 20.8 & Tot. susp. solids & 5.0 & $\mathrm{mg} / \mathrm{L}$ \\
\hline 14 Nov 91 & 20.8 & Turbidity & 1.3 & NTU \\
\hline 15 Nov 91 & 20.8 & Tot. susp. solids & 7.0 & $\mathrm{mg} / \mathrm{L}$ \\
\hline 15 Nov 91 & 20.8 & Turbidity & 1.9 & NTU \\
\hline 18 Nov 91 & 20.8 & Turbidity & 1.4 & NTU \\
\hline 18 Nov 91 & 20.8 & Tot. susp. solids & 5.0 & $\mathrm{mg} / \mathrm{L}$ \\
\hline 19 Nov 91 & 14.5 & Turbidity & 1.7 & NTU \\
\hline 19 Nov 91 & 14.5 & Tot. susp. solids & 5.0 & $\mathrm{mg} / \mathrm{L}$ \\
\hline 19 Nov 91 & 20.8 & Turbidity & 1.4 & NTU \\
\hline 19 Nov 91 & 20.8 & Tot. susp. solids & 5.0 & $\mathrm{mg} / \mathrm{L}$ \\
\hline 20 Nov 91 & 20.8 & Turbidity & 1.4 & NTU \\
\hline 20 Nov 91 & 20.8 & Tot. susp. solids & 5.0 & $\mathrm{mg} / \mathrm{L}$ \\
\hline 21 Nov 91 & 14.5 & Turbidity & 1.2 & NTU \\
\hline 21 Nov 91 & 14.5 & Tot. susp. solids & 5.0 & $\mathrm{mg} / \mathrm{L}$ \\
\hline
\end{tabular}


Table A.2 (continued)

\begin{tabular}{|c|c|c|c|c|}
\hline Date & $\begin{array}{l}\text { River } \\
\text { mile }\end{array}$ & Analyte & Result & Units \\
\hline 02 Dec 91 & 14.5 & Turbidity & 2.5 & NTU \\
\hline $02 \operatorname{Dec} 91$ & 14.5 & Tot. susp. solids & 6.0 & $\mathrm{mg} / \mathrm{L}$ \\
\hline $02 \operatorname{Dec} 91$ & 20.8 & Turbidity & 20.0 & NTU \\
\hline 02 Dec 91 & 20.8 & Tot. susp. solids & 37.0 & $\mathrm{mg} / \mathrm{L}$ \\
\hline 03 Dec 91 & 14.5 & Tot. susp. solids & 11.0 & $\mathrm{mg} / \mathrm{L}$ \\
\hline 03 Dec 91 & 14.5 & Turbidity & 7.2 & NTU \\
\hline $03 \operatorname{Dec} 91$ & 20.8 & Tot. susp. solids & 24.0 & $\mathrm{mg} / \mathrm{L}$ \\
\hline 03 Dec 91 & 20.8 & Turbidity & 18.0 & NTU \\
\hline 04 Dec 91 & 14.5 & Tot. susp. solids & 10.0 & $\mathrm{mg} / \mathrm{L}$ \\
\hline $04 \operatorname{Dec} 91$ & 14.5 & Turbidity & 6.8 & NTU \\
\hline $04 \operatorname{Dec} 91$ & 20.8 & Tot. susp. solids & 13.0 & $\mathrm{mg} / \mathrm{L}$ \\
\hline $04 \operatorname{Dec} 91$ & 20.8 & Turbidity & 7.9 & NTU \\
\hline $05 \operatorname{Dec} 91$ & 14.5 & Tot. susp. solids & 16.0 & $\mathrm{mg} / \mathrm{L}$ \\
\hline $05 \operatorname{Dec} 91$ & 14.5 & Turbidity & 13.0 & NTU \\
\hline 05 Dec 91 & 20.8 & Tot. susp. solids & 12.0 & $\mathbf{m g} / \mathbf{L}$ \\
\hline 05 Dec 91 & 20.8 & Turbidity & 10.0 & NTU \\
\hline $06 \operatorname{Dec} 91$ & 14.5 & Turbidity & 20.0 & NTU \\
\hline 06 Dec 91 & 14.5 & Tot. susp. solids & 21.0 & $\mathrm{mg} / \mathrm{L}$ \\
\hline $06 \operatorname{Dec} 91$ & 20.8 & Tot. susp. solids & 30.0 & $\mathbf{m g} / \mathbf{L}$ \\
\hline $06 \operatorname{Dec} 91$ & 20.8 & Turbidity & 23.0 & NTU \\
\hline 09 Dec 91 & 14.5 & Tot. susp. solids & 8.0 & $\mathrm{mg} / \mathrm{L}$ \\
\hline $09 \operatorname{Dec} 91$ & 14.5 & Turbidity & 10.0 & NTU \\
\hline $09 \operatorname{Dec} 91$ & 20.8 & Turbidity & 10.0 & NTU \\
\hline $09 \operatorname{Dec} 91$ & 20.8 & Tot. susp. solids & 7.0 & $\mathrm{mg} / \mathrm{L}$ \\
\hline $10 \operatorname{Dec} 91$ & 14.5 & Tot. susp. solids & 6.0 & $\mathrm{mg} / \mathrm{L}$ \\
\hline $10 \operatorname{Dec} 91$ & 14.5 & Turbidity & 3.5 & NTU \\
\hline 12 Dec 91 & 14.5 & Turbidity & 2.9 & NTU \\
\hline 12 Dec 91 & 14.5 & Tot. susp. solids & 6.0 & $\mathrm{mg} / \mathrm{L}$ \\
\hline 13 Dec 91 & 14.5 & Tot. susp. solids & 7.0 & $\mathrm{mg} / \mathrm{L}$ \\
\hline $13 \operatorname{Dec} 91$ & 14.5 & Turbidity & 2.3 & NTU \\
\hline $16 \operatorname{Dec} 91$ & 14.5 & Tot. susp. solids & 5.0 & $\mathrm{mg} / \mathrm{L}$ \\
\hline
\end{tabular}


Table A.2 (continued)

\begin{tabular}{|c|c|c|c|c|}
\hline Date & $\begin{array}{c}\text { River } \\
\text { mile }\end{array}$ & Analyte & Result & Units \\
\hline $16 \operatorname{Dec} 91$ & 14.5 & Turbidity & 4.5 & NTU \\
\hline 17 Dec 91 & 14.5 & Turbidity & 8.1 & NTU \\
\hline 17 Dec 91 & 14.5 & Tot. susp. solids & 7.0 & $\mathrm{mg} / \mathrm{L}$ \\
\hline $17 \operatorname{Dec} 91$ & 20.8 & Turbidity & 8.4 & NTU \\
\hline 17 Dec 91 & 20.8 & Tot. susp. solids & 10.0 & $\mathrm{mg} / \mathrm{L}$ \\
\hline $18 \operatorname{Dec} 91$ & 14.5 & Turbidity & 20.0 & NTU \\
\hline $18 \operatorname{Dec} 91$ & 14.5 & Tot. susp. solids & 53.0 & $\mathrm{mg} / \mathrm{L}$ \\
\hline $19 \operatorname{Dec} 91$ & 14.5 & Tot. susp. solids & 8.0 & $\mathrm{mg} / \mathrm{L}$ \\
\hline $19 \operatorname{Dec} 91$ & 14.5 & Turbidity & 5.4 & NTU \\
\hline $20 \operatorname{Dec} 91$ & 14.5 & Turbidity & 14.0 & NTU \\
\hline $20 \operatorname{Dec} 91$ & 14.5 & Tot. susp. solids & 25.0 & $\mathrm{mg} / \mathrm{L}$ \\
\hline $26 \operatorname{Dec} 91$ & 14.5 & Tot. susp. solids & 6.0 & $\mathrm{mg} / \mathrm{L}$ \\
\hline $26 \operatorname{Dec} 91$ & 14.5 & Turbidity & 4.1 & NTU \\
\hline $26 \operatorname{Dec} 91$ & 20.8 & Tot. susp. solids & 8.0 & $\mathbf{m g} / \mathbf{L}$ \\
\hline 26 Dec 91 & 20.8 & Turbidity & 4.7 & NTU \\
\hline $30 \operatorname{Dec} 91$ & 14.5 & Tot. susp. solids & 49.0 & $\mathrm{mg} / \mathrm{L}$ \\
\hline $30 \operatorname{Dec} 91$ & 14.5 & Turbidity & 3.0 & NTU \\
\hline 30 Dec 91 & 20.8 & Turbidity & 3.0 & NTU \\
\hline $30 \operatorname{Dec} 91$ & 20.8 & Tot. susp. solids & 51.0 & $\mathrm{mg} / \mathrm{L}$ \\
\hline 31 Dec 91 & 14.5 & Tot. susp. solids & 36.0 & $\mathrm{mg} / \mathrm{L}$ \\
\hline 2. Dec 91 & 14.5 & Turbidity & 16.0 & NTU \\
\hline $02 \operatorname{Jan} 92$ & 14.5 & Turbidity & 6.0 & NTU \\
\hline $02 \operatorname{Jan} 92$ & 14.5 & Tot. susp. solids & 30.0 & $\mathrm{mg} / \mathrm{L}$ \\
\hline $03 \mathrm{Jan} 92$ & 14.5 & Tot. susp. solids & 12.0 & $\mathrm{mg} / \mathrm{L}$ \\
\hline $03 \operatorname{Jan} 92$ & 14.5 & Turbidity & 5.0 & NTU \\
\hline $06 \operatorname{Jan} 92$ & 14.5 & Tot. susp. solids & 36.0 & $\mathrm{mg} / \mathrm{L}$ \\
\hline $06 \operatorname{Jan} 92$ & 14.5 & Turbidity & 13.0 & NTU \\
\hline $06 \operatorname{Jan} 92$ & 20.8 & Tot. susp. solids & 8.0 & $\mathrm{mg} / \mathrm{L}$ \\
\hline $06 \operatorname{Jan} 92$ & 20.8 & Turbidity & 5.2 & NTU \\
\hline 07 Jan 92 & 14.5 & Turbidity & 18.0 & NTU \\
\hline 07 Jan 92 & 14.5 & Tot. susp. solids & 41.0 & $\mathrm{mg} / \mathrm{L}$ \\
\hline
\end{tabular}


Table A.2 (continued)

\begin{tabular}{|c|c|c|c|c|}
\hline Date & $\begin{array}{c}\text { River } \\
\text { mile }\end{array}$ & Analyte & Result & Units \\
\hline $09 \operatorname{Jan} 92$ & 14.5 & Turbidity & 17.0 & NTU \\
\hline $09 \mathrm{Jan} 92$ & 14.5 & Tot. susp. solids & 30.0 & $\mathrm{mg} / \mathrm{L}$ \\
\hline $13 \operatorname{Jan} 92$ & 14.5 & Tot. susp. solids & 288.0 & $\mathrm{mg} / \mathrm{L}$ \\
\hline $13 \operatorname{Jan} 92$ & 14.5 & Turbidity & 53.0 & NTU \\
\hline $14 \operatorname{Jan} 92$ & 14.5 & Turbidity & 31.0 & NTU \\
\hline $14 \operatorname{Jan} 92$ & 14.5 & Tot. susp. solids & 131.0 & $\mathrm{mg} / \mathrm{L}$ \\
\hline $15 \operatorname{Jan} 92$ & 14.5 & Turbidity & 5.0 & NTU \\
\hline 15 Jan 92 & 14.5 & Tot. susp. solids & 12.0 & $\mathrm{mg} / \mathrm{L}$ \\
\hline $16 \operatorname{Jan} 92$ & 14.5 & Tot. susp. solids & 80.0 & $\mathbf{m g} / \mathrm{L}$ \\
\hline $16 \operatorname{Jan} 92$ & 14.5 & Turbidity & 28.0 & NTU \\
\hline $17 \operatorname{Jan} 92$ & 14.5 & Turbidity & 4.3 & NTU \\
\hline $17 \operatorname{Jan} 92$ & 14.5 & Tot. susp. solids & 10.0 & $\mathrm{mg} / \mathrm{L}$ \\
\hline $20 \mathrm{Jan} 92$ & 14.5 & Turbidity & 25.0 & NTU \\
\hline $20 \operatorname{Jan} 92$ & 14.5 & Tot. susp. solids & 88.0 & $\mathrm{mg} / \mathrm{L}$ \\
\hline $22 \operatorname{Jan} 92$ & 14.5 & Tot. susp. solids & 5.0 & $\mathrm{mg} / \mathrm{L}$ \\
\hline $22 \operatorname{Jan} 92$ & 14.5 & Turbidity & 3.4 & NTU \\
\hline $23 \operatorname{Jan} 92$ & 14.5 & Tot. susp. solids & 20.0 & $\mathrm{mg} / \mathrm{L}$ \\
\hline $23 \operatorname{Jan} 92$ & 14.5 & Turbidity & 10.0 & NTU \\
\hline $24 \operatorname{Jan} 92$ & 14.5 & Turbidity & 5.0 & NTU \\
\hline $24 \operatorname{Jan} 92$ & 14.5 & Tot. susp. solids & 7.0 & $\mathrm{mg} / \mathrm{L}$ \\
\hline 27 Jan 92 & 14.5 & Turbidity & 12.0 & NTU \\
\hline $27 \operatorname{Jan} 92$ & 14.5 & Tot. susp. solids & 27.0 & $\mathrm{mg} / \mathrm{L}$ \\
\hline $28 \operatorname{Jan} 92$ & 14.5 & Turbidity & 11.0 & NTU \\
\hline $28 \operatorname{Jan} 92$ & 14.5 & Tot. susp. solids & 36.0 & mg/L \\
\hline $29 \operatorname{Jan} 92$ & 14.5 & Turbidity & 3.0 & NTU \\
\hline $29 \mathrm{Jan} 92$ & 14.5 & Tot. susp. solids & 5.0 & $\mathrm{mg} / \mathrm{L}$ \\
\hline $30 \operatorname{Jan} 92$ & 14.5 & Turbidity & 3.0 & NTU \\
\hline $30 \mathrm{Jan} 92$ & 14.5 & Tot. susp. solids & 5.0 & $\mathrm{mg} / \mathrm{L}$ \\
\hline 05 Feb 92 & 20.8 & Turbidity & 4.1 & NTU \\
\hline 05 Feb 92 & 20.8 & Tot. susp. solids & 7.0 & $\mathrm{mg} / \mathrm{L}$ \\
\hline 06 Feb 92 & 20.8 & Turbidity & 4.5 & NTU \\
\hline
\end{tabular}


Table A.2 (continued)

\begin{tabular}{|c|c|c|c|c|}
\hline Date & $\begin{array}{c}\text { River } \\
\text { mile }\end{array}$ & Analyte & Result & Units \\
\hline 06 Feb 92 & 20.8 & Tot. susp. solids & 7.0 & $\mathbf{m g} / \mathbf{L}$ \\
\hline 08 Feb 92 & 20.8 & Turbidity & 4.5 & NTU \\
\hline 08 Feb 92 & 20.8 & Tot. susp. solids & 8.0 & $\mathrm{mg} / \mathrm{L}$ \\
\hline 11 Feb 92 & 20.8 & Tot. susp. solids & 19.0 & $\mathbf{m g} / \mathbf{L}$ \\
\hline 11 Feb 92 & 20.8 & Turbidity & 10.0 & NTU \\
\hline 12 Feb 92 & 20.8 & Tot. susp. solids & 7.0 & $\mathrm{mg} / \mathrm{L}$ \\
\hline 12 Feb 92 & 20.8 & Turbidity & 5.0 & NTU \\
\hline 18 Feb 92 & 20.8 & Turbidity & 5.0 & NTU \\
\hline 18 Feb 92 & 20.8 & Tot. susp. solids & 5.0 & $\mathrm{mg} / \mathrm{L}$ \\
\hline 19 Feb 92 & 20.8 & Turbidity & 10.0 & NTU \\
\hline 19 Feb 92 & 20.8 & Tot. susp. solids & 10.0 & $\mathrm{mg} / \mathrm{L}$ \\
\hline 21 Feb 92 & 20.8 & Tot. susp. solids & 8.0 & $\mathrm{mg} / \mathrm{L}$ \\
\hline 21 Feb 92 & 20.8 & Turbidity & 7.0 & NTU \\
\hline 21 Feb 92 & 20.8 & Tot. susp. solids & 8.0 & $\mathbf{m g} / \mathbf{L}$ \\
\hline 25 Feb 92 & 20.8 & Turbidity & 10.0 & NTU \\
\hline 25 Feb 92 & 20.8 & Tot. susp. solids & 14.0 & $\mathrm{mg} / \mathrm{L}$ \\
\hline 26 Feb 92 & 20.8 & Tot. susp. solids & 16.0 & $\mathrm{mg} / \mathrm{L}$ \\
\hline 26 Feb 92 & 20.8 & Turbidity & 14.0 & NTU \\
\hline 27 Feb 92 & 14.5 & Turbidity & 8.0 & NTU \\
\hline 27 Feb 92 & 14.5 & Tot. susp. solids & 6.0 & $\mathrm{mg} / \mathrm{L}$ \\
\hline 27 Feb 92 & 20.8 & Tot. susp. solids & 17.0 & $\mathrm{mg} / \mathrm{L}$ \\
\hline 27 Feb 92 & 20.8 & Turbidity & 21.0 & NTU \\
\hline 03 Mar 92 & 14.5 & Tot. susp. solids & 9.5 & $\mathrm{mg} / \mathrm{L}$ \\
\hline 03 Mar 92 & 14.5 & Turbidity & 6.0 & NTU \\
\hline 03 Mar 92 & 20.8 & Tot. susp. solids & 11.0 & $\mathrm{mg} / \mathrm{L}$ \\
\hline 03 Mar 92 & 20.8 & Turbidity & 8.0 & NTU \\
\hline 05 Mar 92 & 14.5 & Tot. susp. solids & 5.0 & $\mathrm{mg} / \mathrm{L}$ \\
\hline 05 Mar 92 & 14.5 & Turbidity & 4.0 & NTU \\
\hline 05 Mar 92 & 20.8 & Turbidity & 6.0 & NTU \\
\hline $05 \operatorname{Mar} 92$ & 20.8 & Tot. susp. solids & 8.0 & $\mathrm{mg} / \mathrm{L}$ \\
\hline 06 Mar 92 & 14.5 & Turbidity & 8.5 & NTU \\
\hline
\end{tabular}


Table A.2 (continued)

\begin{tabular}{|c|c|c|c|c|}
\hline Date & $\begin{array}{l}\text { River } \\
\text { mile }\end{array}$ & Analyte & Result & Units \\
\hline $06 \operatorname{Mar} 92$ & 14.5 & Tot. susp. solids & 18.0 & $\mathrm{mg} / \mathrm{L}$ \\
\hline 06 Mar 92 & 20.8 & Tot. susp. solids & 10.0 & $\mathrm{mg} / \mathrm{L}$ \\
\hline 06 Mar 92 & 20.8 & Turbidity & 7.5 & NTU \\
\hline $10 \operatorname{Mar} 92$ & 14.5 & Turbidity & 4.3 & NTU \\
\hline $10 \operatorname{Mar} 92$ & 14.5 & Tot. susp. solids & 7.0 & $\mathrm{mg} / \mathrm{L}$ \\
\hline $10 \operatorname{Mar} 92$ & 20.8 & Turbidity & 10.0 & NTU \\
\hline $10 \operatorname{Mar} 92$ & 20.8 & Tot. susp. solids & 13.0 & $\mathrm{mg} / \mathrm{L}$ \\
\hline $17 \operatorname{Mar} 92$ & 14.5 & Turbidity & 5.3 & NTU \\
\hline $17 \operatorname{Mar} 92$ & 14.5 & Tot. susp. solids & 9.0 & mg/L \\
\hline 18 Mar 92 & 14.5 & Turbidity & 6.1 & NTU \\
\hline 18 Mar 92 & 14.5 & Tot. susp. solids & 9.0 & $\mathrm{mg} / \mathrm{L}$ \\
\hline $18 \operatorname{Mar} 92$ & 20.8 & Turbidity & 7.6 & NTU \\
\hline $18 \operatorname{Mar} 92$ & 20.8 & Tot. susp. solids & 14.0 & $\mathrm{mg} / \mathrm{L}$ \\
\hline $19 \operatorname{Mar} 92$ & 14.5 & Turbidity & 10.0 & NTU \\
\hline 19 Mar 92 & 14.5 & Tot. susp. solids & 28.0 & $\mathrm{mg} / \mathrm{L}$ \\
\hline 19 Mar 92 & 20.8 & Turbidity & 11.0 & NTU \\
\hline 20 Mar 92 & 14.5 & Turbidity & 6.0 & NTU \\
\hline 20 Mar 92 & 14.5 & Tot. susp. solids & 9.0 & $\mathbf{m g} / \mathbf{L}$ \\
\hline 20 Mar 92 & 20.8 & Tot. susp. solids & 19.0 & $\mathrm{mg} / \mathrm{L}$ \\
\hline $20 \operatorname{Mar} 92$ & 20.8 & Turbidity & 15.0 & NTU \\
\hline $24 \operatorname{Mar} 92$ & 14.5 & Turbidity & 10.0 & NTU \\
\hline $24 \operatorname{Mar} 92$ & 14.5 & Tot. susp. solids & 16.0 & $\mathrm{mg} / \mathrm{L}$ \\
\hline $24 \operatorname{Mar} 92$ & 20.8 & Turbidity & 9.8 & NTU \\
\hline $24 \operatorname{Mar} 92$ & 20.8 & Tot. susp. solids & 10.0 & $\mathrm{mg} / \mathrm{L}$ \\
\hline $25 \operatorname{Mar} 92$ & 14.5 & Turbidity & 5.9 & NTU \\
\hline $25 \operatorname{Mar} 92$ & 14.5 & Tot. susp. solids & 8.0 & $\mathrm{mg} / \mathrm{L}$ \\
\hline $25 \operatorname{Mar} 92$ & 20.8 & Turbidity & 9.5 & NTU \\
\hline $25 \operatorname{Mar} 92$ & 20.8 & Tot. susp. solids & 11.0 & $\mathrm{mg} / \mathrm{L}$ \\
\hline $26 \operatorname{Mar} 92$ & 14.5 & Turbidity & 12.0 & NTL \\
\hline $26 \operatorname{Mar} 92$ & 14.5 & Tot. susp. solids & 42.0 & $\mathrm{mg} / \mathrm{L}$ \\
\hline 26 Mar 92 & 20.8 & Tot. susp. solids & 24.0 & $\mathrm{mg} / \mathrm{L}$ \\
\hline
\end{tabular}


Table A.2 (continued)

\begin{tabular}{|c|c|c|c|c|}
\hline Date & $\begin{array}{c}\text { River } \\
\text { mile }\end{array}$ & Analyte & Result & Units \\
\hline $26 \operatorname{Mar} 92$ & 20.8 & Turbidity & 10.5 & NTU \\
\hline $27 \operatorname{Mar} 92$ & 20.8 & Turbidity & 12.5 & NTU \\
\hline $27 \operatorname{Mar} 92$ & 20.8 & Tot. susp. solids & 33.0 & $\mathrm{mg} / \mathrm{L}$ \\
\hline $31 \operatorname{Mar} 92$ & 14.5 & Turbidity & 19.0 & NTU \\
\hline 31 Mar 92 & 14.5 & Tot. susp. solids & 72.0 & $\mathrm{mg} / \mathrm{L}$ \\
\hline $31 \operatorname{Mar} 92$ & 20.8 & Turbidity & 7.5 & NTU \\
\hline $31 \operatorname{Mar} 92$ & 20.8 & Tot. susp. solids & 11.0 & $\mathrm{mg} / \mathrm{L}$ \\
\hline 01 Apr 92 & 14.5 & Tot. susp. solids & 72.0 & $\mathrm{mg} / \mathrm{L}$ \\
\hline 01 Apr 92 & 14.5 & Turbidity & 21.0 & NTU \\
\hline 01 Apr 92 & 20.8 & Tot. susp. solids & 5.0 & $\mathrm{mg} / \mathrm{L}$ \\
\hline 01 Apr 92 & 20.8 & Turbidity & 28.0 & NTU \\
\hline 02 Apr 92 & 14.5 & Turbidity & 4.6 & NTU \\
\hline 02 A.pr 92 & 14.5 & Tot. susp. solids & 7.0 & $\mathrm{mg} / \mathrm{L}$ \\
\hline 02 Apr 92 & 20.8 & Turbidity & 10.0 & NTU \\
\hline 02 Apr 92 & 20.8 & Tot. susp. solids & 13.0 & $\mathrm{mg} / \mathrm{L}$ \\
\hline 07 Apr 92 & 14.5 & Turbidity & 11.5 & NTU \\
\hline 07 Apr 92 & 14.5 & Tot. susp. solids & 35.0 & $\mathrm{mg} / \mathrm{L}$ \\
\hline 07 Apr 92 & 20.8 & Turbidity & 9.0 & NTU \\
\hline 07 Apr 92 & 20.8 & Tot. susp. solids & 10.0 & $\mathrm{mg} / \mathrm{L}$ \\
\hline 08 Apr 92 & 14.5 & Tot. susp. solids & 12.0 & $\mathrm{mg} / \mathrm{L}$ \\
\hline 08 Apr 92 & 14.5 & Turbidity & 5.0 & NTU \\
\hline 08 Apr 92 & 20.8 & Tot. susp. solids & 14.0 & $\mathrm{mg} / \mathrm{L}$ \\
\hline 08 Apr 92 & 20.8 & Turbidity & 9.0 & NTU \\
\hline 09 Apr 92 & 14.5 & Turbidity & 10.0 & NTU \\
\hline 09 Apr 92 & 14.5 & Tot. susp. solids & 32.0 & $\mathrm{mg} / \mathrm{L}$ \\
\hline 09 Apr 92 & 20.8 & Tot. susp. solids & 5.0 & $\mathrm{mg} / \mathrm{L}$ \\
\hline 09 Apr 92 & 20.8 & Turbidity & 5.5 & NrU \\
\hline 10 Apr 92 & 14.5 & Turbidity & 4.2 & NTU \\
\hline 10 Apr 92 & 14.5 & Tot. susp. solids & 12.0 & $\mathrm{mg} / \mathrm{L}$ \\
\hline 10 Apr 92 & 20.8 & Tot. susp. solids & 11.0 & $\mathrm{mg} / \mathrm{L}$ \\
\hline $10 \mathrm{Apr} 92$ & 20.8 & Turbidity & 6.0 & NTU \\
\hline
\end{tabular}


Table A.2 (continued)

\begin{tabular}{|c|c|c|c|c|}
\hline Date & $\begin{array}{c}\text { River } \\
\text { mile }\end{array}$ & Analyte & Result & Units \\
\hline 14 Apr 92 & 14.5 & Tot. susp. solids & 19.0 & $\mathrm{mg} / \mathrm{L}$ \\
\hline 14 Apr 92 & 14.5 & Turbidity & 7.8 & NTU \\
\hline 15 Apr 92 & 14.5 & Turbidity & 6.0 & NTU \\
\hline 15 Apr 92 & 14.5 & Tot. susp. solids & 16.0 & $\mathrm{mg} / \mathrm{L}$ \\
\hline 15 Apr 92 & 20.8 & Turbidity & 26.0 & NTU \\
\hline 15 Apr 92 & 20.8 & Tot. susp. solids & 22.0 & $\mathrm{mg} / \mathrm{L}$ \\
\hline 16 Apr 92 & 14.5 & Tot. susp. solids & 33.0 & $\mathrm{mg} / \mathrm{L}$ \\
\hline 16 Apr 92 & 14.5 & Turbidity & 12.0 & NTU \\
\hline 16 Apr 92 & 20.8 & Tot. susp. solids & 12.0 & $\mathrm{mg} / \mathrm{L}$ \\
\hline 16 Apr 92 & 20.8 & Turbidity & 15.0 & NTU \\
\hline 22 Apr 92 & 14.5 & Tot. susp. solids & 21.0 & $\mathrm{mg} / \mathrm{L}$ \\
\hline 22 Apr 92 & 14.5 & Turbidity & 8.0 & NTU \\
\hline 22 Apr 92 & 20.8 & Tot. susp. solids & 5.0 & $\mathrm{mg} / \mathrm{L}$ \\
\hline 22 Apr 92 & 20.8 & Turbidity & 3.9 & NTU \\
\hline 29 Apr 92 & 14.5 & Tot. susp. solids & 11.0 & $\mathrm{mg} / \mathrm{L}$ \\
\hline 29 Apr 92 & 14.5 & Turbidity & 4.8 & NiU \\
\hline 29 Apr 92 & 20.8 & Turbidity & 3.0 & NTU \\
\hline 29 Apr 92 & 20.8 & Tot. susp. solids & 7.0 & mg/L \\
\hline 06 May 92 & 14.5 & Turbidity & 6.3 & NTU \\
\hline 06 May 92 & 14.5 & Tot. susp. solids & 21.0 & $\mathrm{mg} / \mathrm{L}$ \\
\hline 06 May 92 & 20.8 & Turbidity & 1.9 & NTU \\
\hline 06 May 92 & 20.8 & Tot. susp. solids & 5.0 & $\mathrm{mg} / \mathrm{L}$ \\
\hline 13 May 92 & 14.5 & Turbidity & 18.0 & NTI \\
\hline 13 May 92 & 14.5 & Tot. susp. solids & 64.0 & mgh \\
\hline 13 May 92 & 20.8 & Turbidity & 2.0 & $\because \bar{L} U$ \\
\hline 13 May 92 & 20.8 & Tot. susp. solids & 5.0 & $\mathrm{mg} / \mathrm{L}$ \\
\hline 20 May 92 & 14.5 & Turbidity & 2.3 & NTU \\
\hline 20 May 92 & 14.5 & Tot. susp. solids & 7.0 & $\mathrm{mg} / \mathrm{L}$ \\
\hline 20 May 92 & 20.8 & Turbidity & 1.6 & NTU \\
\hline 20 May 92 & 20.8 & Tot. susp. solids & 5.0 & $\mathrm{mg} / \mathrm{L}$ \\
\hline 28 May 92 & 14.5 & Turbidity & 12.0 & NTU \\
\hline
\end{tabular}


Table A.2 (continued)

\begin{tabular}{|c|c|c|c|c|}
\hline Date & $\begin{array}{c}\text { River } \\
\text { mile }\end{array}$ & Analyte & Result & Units \\
\hline 28 May 92 & 14.5 & Tot. susp. solids & 72.0 & $\mathrm{mg} / \mathrm{L}$ \\
\hline 28 May 92 & 20.8 & Turbidity & 1.7 & NTU \\
\hline 28 May 92 & 20.8 & Tot. susp. solids & 5.0 & $\mathrm{mg} / \mathrm{L}$ \\
\hline 04 Jun 92 & 14.5 & Turbidity & 3.6 & NTU \\
\hline 04 Jun 92 & 14.5 & Tot. susp. solids & 25.0 & $\mathrm{mg} / \mathrm{L}$ \\
\hline 04 Jun 92 & 20.8 & Tot. susp. solids & 5.0 & $\mathrm{mg} / \mathrm{L}$ \\
\hline $04 \mathrm{~J} \in \operatorname{n} 92$ & 20.8 & Turbidity & 1.4 & NTU \\
\hline 10 Jun 92 & 14.5 & Tot. susp. solids & 5.0 & $\mathbf{m g} / \mathbf{L}$ \\
\hline 10 Jun 92 & 14.5 & Turbidity & 2.5 & NTU \\
\hline 10 Jun 92 & 20.8 & Tot. susp. solids & 5.0 & $\mathbf{m g} / \mathrm{L}$ \\
\hline 10 Jun 92 & 20.8 & Turbidity & 1.7 & NTU \\
\hline 17 Jun 92 & 14.5 & Tot. susp. solids & 6.0 & mgll \\
\hline 17 Jun 92 & 14.5 & Turbidity & 3.2 & NTU \\
\hline 17 Jun 92 & 20.8 & Turbidity & 2.7 & NTU \\
\hline 17 Jun 92 & 20.8 & Tot. susp. solids & 5.0 & $\mathrm{mg} / \mathrm{L}$ \\
\hline 24 Jun 92 & 14.5 & Tot. susp. soliuis & 6.0 & $\mathrm{mg} / \mathrm{L}$ \\
\hline 24 Jun 92 & 14.5 & Turbidity & 5.5 & NTU \\
\hline 24 Jun 92 & 20.8 & Turbidity & 2.5 & NTU \\
\hline 24 Jun 92 & 20.8 & Tot. susp. solids & 5.0 & $\mathrm{mg} / \mathrm{L}$ \\
\hline $01 \mathrm{Jul} 92$ & 14.5 & Tot. susp. solids & 37.0 & $\mathrm{mg} / \mathrm{L}$ \\
\hline $01 \mathrm{Jul} 92$ & 14.5 & Turbidity & 9.0 & NTU \\
\hline 01 Jul 92 & 20.8 & Tot. susp. solids & 5.0 & $\mathbf{m g} / \mathbf{L}$ \\
\hline $01 \mathrm{Jul} 92$ & 20.8 & Turbidity & 3.0 & NTU \\
\hline 08 Jul 92 & 14.5 & Tot. susp, solids & 7.0 & $\mathrm{mg} / \mathrm{L}$ \\
\hline $08 \mathrm{Jul} 92$ & 14.5 & Turbidity & 3.9 & NTU \\
\hline $08 \mathrm{Jul} 92$ & 20.8 & Turbidity & 4.7 & NTU \\
\hline $08 \mathrm{Jul} 92$ & 20.8 & Tot. susp. solids & 5.0 & $\mathrm{mg} / \mathrm{L}$ \\
\hline 15 Jul 92 & 14.5 & Tot. susp. solids & 5.0 & $\mathrm{mg} / \mathrm{L}$ \\
\hline 15 Jul 92 & 14.5 & Turbidity & 3.1 & NTU \\
\hline $15 \mathrm{Jul} 92$ & 20.8 & Turbidity & 3.8 & NTU \\
\hline 15 Jul 92 & 20.8 & Tot. susp. solids & 9.0 & $\mathrm{mg} / \mathrm{L}$ \\
\hline
\end{tabular}


Table A.2 (continued)

\begin{tabular}{cclcc}
\hline Date & $\begin{array}{c}\text { River } \\
\text { mile }\end{array}$ & \multicolumn{1}{c}{ Analyte } & Result & Units \\
\hline 23 Jul 92 & 14.5 & Turbidity & 55.0 & $\mathrm{NTU}$ \\
23 Jul 92 & 14.5 & Tot. susp. solids & 363.0 & $\mathrm{mg} / \mathrm{L}$ \\
23 Jul 92 & 20.8 & Tot. susp. solids & 5.0 & $\mathrm{mg} / \mathrm{L}$ \\
23 Jul 92 & 20.8 & Turbidity & 2.9 & $\mathrm{NTU}$ \\
29 Jul 92 & 20.8 & Tot. susp. solids & 6.0 & $\mathrm{mg} /$ \\
29 Jul 92 & 20.8 & Tot. susp. solids & 5.0 & $\mathrm{mg} /$ \\
29 Jul 92 & 20.8 & Turbidity & 2.5 & $\mathrm{NTU}$ \\
29 Jul 92 & 20.8 & Turbidity & 4.0 & $\mathrm{NTU}$ \\
\hline
\end{tabular}


Table A.3. Composite sampler particle-associated ${ }^{17} \mathrm{Cs}$ results

\begin{tabular}{|c|c|c|c|c|c|c|c|}
\hline Date & $\begin{array}{c}\text { River } \\
\text { mile }\end{array}$ & $\begin{array}{l}\text { TSP } \\
\text { result }\end{array}$ & Error & Units & $\begin{array}{l}{ }^{137} \mathrm{Cs} \\
\text { result }\end{array}$ & $\begin{array}{l}\text { Rad } \\
\text { error }\end{array}$ & Unit \\
\hline $18 \operatorname{Sep} 91$ & 20.8 & & & & 1.92 & 1.22 & $\mathrm{pCi} / \mathrm{L}$ \\
\hline 18 Sep 91 & 20.8 & 5.0 & & $\mathrm{mg} / \mathrm{L}$ & 135.15 & 0.00 & $\mathrm{pCi} / \mathrm{g}$ \\
\hline $19 \operatorname{Sep} 91$ & 20.8 & & & & 0.22 & 1.24 & $\mathrm{pCi} / \mathrm{L}$ \\
\hline $19 \operatorname{Sep} 91$ & 20.8 & 5.0 & & ing/L & 43.25 & 248.68 & $\mathrm{pCi} / \mathrm{g}$ \\
\hline 19 Sep 91 & 14.5 & & & & 1.00 & 0.59 & $\mathrm{pCi} / \mathrm{L}$ \\
\hline $19 \operatorname{Sep} 91$ & 14.5 & 11.0 & & $\mathrm{mg} / \mathrm{L}$ & 90.92 & 54.06 & $\mathrm{pCi} / \mathrm{g}$ \\
\hline $20 \operatorname{Sep} 91$ & 20.8 & & & & 1.73 & 0.70 & $\mathrm{pCi} / \mathrm{L}$ \\
\hline $20 \operatorname{Sep} 91$ & 20.8 & 5.0 & & $\mathrm{mg} / \mathrm{L}$ & 345.98 & 140.56 & $\mathrm{pCi} / \mathrm{g}$ \\
\hline $23 \operatorname{Sep} 91$ & 20.8 & & & & 1.08 & 1.62 & $\mathrm{pCi} / \mathrm{L}$ \\
\hline 23 Sep 91 & 20.8 & 5.0 & & $\mathrm{mg} / \mathrm{L}$ & 216.24 & 324.36 & $\mathrm{pCi} / \mathrm{g}$ \\
\hline $24 \operatorname{Sep} 91$ & 20.8 & & & & 2.00 & 0.89 & $\mathrm{pCi} / \mathrm{L}$ \\
\hline 24 Sep 91 & 20.8 & 5.0 & & $\mathrm{mg} / \mathrm{L}$ & 400.04 & 178.40 & $\mathrm{pCi} / \mathrm{g}$ \\
\hline $24 \operatorname{Sep} 91$ & 14.5 & & & & -0.05 & 1.30 & $\mathrm{pCi} / \mathrm{L}$ \\
\hline $24 \operatorname{Sep} 91$ & 14.5 & 5.0 & & $\mathbf{m g} / \mathbf{L}$ & -10.81 & 259.49 & $\mathrm{pCi} / \mathrm{g}$ \\
\hline $25 \operatorname{Sep} 91$ & 20.8 & & & & 1.65 & 0.81 & $\mathrm{pCi} / \mathrm{L}$ \\
\hline $25 \operatorname{Sep} 91$ & 20.8 & 5.0 & & $\mathrm{mg} / \mathrm{L}$ & 329.77 & 162.18 & $\mathrm{pCi} / \mathrm{g}$ \\
\hline 25 Sep 91 & 14.5 & & & & -0.08 & 0.59 & $\mathrm{pCi} / \mathrm{L}$ \\
\hline 25 Sep 91 & 14.5 & 5.0 & & $\mathrm{mg} / \mathrm{L}$ & -16.22 & 118.93 & $\mathrm{pCi} / \mathrm{g}$ \\
\hline $26 \operatorname{Sep} 91$ & 20.8 & & & & 4.05 & 1.35 & $\mathrm{pCi} / \mathrm{L}$ \\
\hline 26 Sep 91 & 20.8 & 5.0 & & $\mathrm{mg} / \mathrm{L}$ & 810.90 & 270.30 & $\mathrm{pCi} / \mathrm{g}$ \\
\hline $26 \operatorname{Sep} 91$ & 14.5 & & & & 0.54 & 0.22 & $\mathrm{pCi} / \mathrm{L}$ \\
\hline 26 Sep 91 & 14.5 & 5.0 & & $\mathrm{mg} / \mathrm{L}$ & 108.12 & 43.25 & $\mathrm{pCi} / \mathrm{g}$ \\
\hline $27 \operatorname{Sep} 91$ & 20.8 & & & & 1.27 & 1.03 & $\mathrm{pCi} / \mathrm{L}$ \\
\hline $27 \operatorname{Sep} 91$ & 20.8 & 5.0 & & $\mathrm{mg} / \mathrm{L}$ & 254.08 & 205.43 & $\mathrm{pCi} / \mathrm{g}$ \\
\hline 27 Sep 91 & 14.5 & & & & 0.68 & 0.78 & $\mathrm{pCi} / \mathrm{L}$ \\
\hline 27 Sep 91 & 14.5 & 5.0 & & $\mathrm{mg} / \mathrm{L}$ & 135.15 & 156.77 & $\mathrm{pCi} / \mathrm{g}$ \\
\hline 30 Sep 91 & 20.8 & & & & 0.35 & 0.65 & $\mathrm{pCi} / \mathrm{L}$ \\
\hline 30 Sep 91 & 20.8 & 5.0 & & $\mathrm{mg} / \mathrm{L}$ & 70.28 & 129.74 & $\mathrm{pCi} / \mathrm{g}$ \\
\hline $30 \operatorname{Sep} 91$ & 14.5 & & & & 0.46 & 0.19 & $\mathrm{pCi} / \mathrm{L}$ \\
\hline 30 Sep 91 & 14.5 & 5.0 & & $\mathrm{mg} / \mathrm{L}$ & 91.90 & 37.84 & $\mathrm{pCi} / \mathrm{g}$ \\
\hline
\end{tabular}




\section{A-23}

Table A.3 (continued)

\begin{tabular}{|c|c|c|c|c|c|c|c|}
\hline Date & $\begin{array}{l}\text { River } \\
\text { mile }\end{array}$ & $\begin{array}{l}\text { TSP } \\
\text { result }\end{array}$ & Error & Units & $\begin{array}{l}{ }^{137} \mathrm{Cs} \\
\text { result }\end{array}$ & $\begin{array}{l}\text { Rad } \\
\text { error }\end{array}$ & Unit \\
\hline 01 Oct 91 & 20.8 & & & & 0.84 & 1.05 & $\mathrm{pCi} / \mathrm{L}$ \\
\hline 01 Oct 91 & 20.8 & 5.0 & & $\mathrm{mg} / \mathrm{L}$ & 167.59 & 210.83 & $\mathrm{pCi} / \mathrm{g}$ \\
\hline 01 Oct 91 & 14.5 & & & & -0.32 & 0.78 & $\mathrm{pCI} / \mathrm{L}$ \\
\hline 01 Oct 91 & 14.5 & 5.0 & & $\mathrm{mg} / \mathrm{L}$ & -64.87 & 156.77 & $\mathrm{pCi} / \mathrm{g}$ \\
\hline 02 Oct 91 & 20.8 & & & & 0.73 & 1.32 & $\mathrm{pCi} / \mathrm{L}$ \\
\hline 02 Oct 91 & 20.8 & 5.0 & & $\mathrm{mg} / \mathrm{L}$ & 145.96 & 264.89 & $\mathrm{pCi} / \mathrm{g}$ \\
\hline 02 Oct 91 & 14.5 & & & & 0.05 & 0.81 & $\mathrm{pCi} / \mathrm{L}$ \\
\hline 02 Oct 91 & 14.5 & 5.0 & & $\mathrm{mg} / \mathrm{L}$ & 10.81 & 162.18 & $\mathrm{pCi} / \mathrm{g}$ \\
\hline 03 Oct 91 & 20.8 & & & & 0.32 & 0.73 & $\mathrm{pCi} / \mathrm{L}$ \\
\hline 03 Oct 91 & 20.8 & 5.0 & & $\mathrm{mg} / \mathrm{L}$ & 64.87 & 145.96 & $\mathrm{pCi} / \mathrm{g}$ \\
\hline 03 Oct 91 & 14.5 & & & & 0.35 & 0.84 & $\mathrm{pCi} / \mathrm{L}$ \\
\hline 03 Oct 91 & 14.5 & 5.0 & & $\mathrm{mg} / \mathrm{L}$ & 70.28 & 167.59 & $\mathrm{pCi} / \mathrm{g}$ \\
\hline 04 Oct 91 & 20.8 & & & & 0.14 & 1.05 & $\mathrm{pCi} / \mathrm{L}$ \\
\hline 04 Oct 91 & 20.8 & 5.0 & & $\mathrm{mg} / \mathrm{L}$ & 27.03 & 210.83 & $\mathrm{pCi} / \mathrm{g}$ \\
\hline 04 Oct 91 & 14.5 & & & & 0.14 & 1.30 & $\mathrm{pCi} / \mathrm{L}$ \\
\hline 04 Oct 91 & 14.5 & 5.0 & & $\mathrm{mg} / \mathrm{L}$ & 27.03 & 259.49 & $\mathrm{pCi} / \mathrm{g}$ \\
\hline 07 Oct 91 & 20.8 & & & & -0.38 & 1.35 & $\mathrm{pCi} / \mathrm{L}$ \\
\hline 07 Oct 91 & 20.8 & 5.0 & & $\mathbf{m g} / \mathrm{L}$ & -75.68 & 270.30 & $\mathrm{pCi} / \mathrm{g}$ \\
\hline 07 Oct 91 & 14.5 & & & & 0.43 & 0.70 & $\mathrm{pCi} / \mathrm{L}$ \\
\hline 07 Oct 91 & 14.5 & 5.0 & & $\mathrm{mg} / \mathrm{L}$ & 86.50 & 140.56 & $\mathrm{pCi} / \mathrm{g}$ \\
\hline 29 Oct 91 & 20.8 & & & & 0.27 & 0.65 & $\mathrm{pCi} / \mathrm{L}$ \\
\hline 29 Oct 91 & 20.8 & 2.0 & & $\mathrm{mg} / \mathrm{L}$ & 135.15 & 324.36 & $\mathrm{pCi} / \mathrm{g}$ \\
\hline 29 Oct 91 & 14.5 & & & & 0.54 & 0.59 & $\mathrm{pCi} / \mathrm{L}$ \\
\hline 29 Oct 91 & 14.5 & 5.0 & & $\mathrm{mg} / \mathrm{L}$ & 108.12 & 118.93 & $\mathrm{pCi} / \mathrm{g}$ \\
\hline 30 Oct 91 & 20.8 & & & & 3.24 & 0.54 & $\mathrm{pCi} / \mathrm{L}$ \\
\hline 30 Oct 91 & 20.8 & 6.0 & & $\mathrm{mg} / \mathrm{L}$ & 540.60 & 90.10 & $\mathrm{pCi} / \mathrm{g}$ \\
\hline 31 Oct 91 & 20.8 & & & & 0.35 & 0.89 & $\mathrm{pCi} / \mathrm{L}$ \\
\hline 31 Oct 91 & 20.8 & 5.0 & & $\mathbf{m g} / \mathbf{L}$ & 70.28 & 178.40 & $\mathrm{pCi} / \mathrm{g}$ \\
\hline 31 Oct 91 & 14.5 & & & & 0.22 & 0.51 & $\mathrm{pCi} / \mathrm{L}$ \\
\hline 31 Oct 91 & 14.5 & 5.0 & & $\mathrm{mg} / \mathrm{L}$ & 43.25 & 102.71 & $\mathrm{pCi} / \mathrm{g}$ \\
\hline 01 Nov 91 & 20.8 & & & & 0.14 & 0.86 & $\mathrm{pCi} / \mathrm{L}$ \\
\hline
\end{tabular}


Table A.3 (continued)

\begin{tabular}{|c|c|c|c|c|c|c|c|}
\hline Date & $\begin{array}{c}\text { River } \\
\text { mile }\end{array}$ & $\begin{array}{l}\text { TSP } \\
\text { result }\end{array}$ & Error & Units & $\begin{array}{l}{ }^{137} \mathrm{Cs} \\
\text { result }\end{array}$ & $\begin{array}{l}\text { Rad } \\
\text { error }\end{array}$ & Unit \\
\hline 01 Nov 91 & 20.8 & 5.0 & & $\mathrm{mg} / \mathrm{L}$ & 27.03 & 172.99 & $\mathrm{pCi} / \mathrm{g}$ \\
\hline 04 Nov 91 & 20.8 & & & & 0.41 & 0.81 & $\mathrm{pCi} / \mathrm{L}$ \\
\hline 04 Nov 91 & 20.8 & 5.0 & & $\mathrm{mg} / \mathrm{L}$ & 81.09 & 162.18 & $\mathrm{pCi} / \mathrm{g}$ \\
\hline 05 Nov 91 & 20.8 & & & & -0.11 & 1.22 & $\mathrm{pCi} / \mathrm{L}$ \\
\hline 05 Nov 91 & 20.8 & 19.0 & & $\mathrm{mg} / \mathrm{L}$ & -5.69 & 64.02 & $\mathrm{pCi} / \mathrm{g}$ \\
\hline 05 Nov 91 & 14.5 & & & & 0.49 & 0.73 & $p \mathrm{Ci} / \mathrm{L}$ \\
\hline 05 Nov 91 & 14.5 & 6.0 & & $\mathrm{mg} / \mathrm{L}$ & 81.09 & 121.64 & $\mathrm{pCi} / \mathrm{g}$ \\
\hline 06 Nov 91 & 20.8 & & & & 22.16 & 4.87 & $\mathrm{pCi} / \mathrm{L}$ \\
\hline 06 Nov 91 & 20.8 & 51.0 & & $\mathrm{mg} / \mathrm{L}$ & 434.60 & 95.40 & $\mathrm{pCi} / \mathrm{g}$ \\
\hline 06 Nov 91 & 20.8 & & & & 2.38 & 0.68 & $\mathrm{pCi} / \mathrm{L}$ \\
\hline 06 Nov 91 & 20.8 & 5.0 & & $\mathrm{mg} / \mathrm{L}$ & 475.73 & 135.15 & $\mathrm{pCi} / \mathrm{g}$ \\
\hline 06 Nov 91 & 14.5 & & & & 0.46 & 0.65 & $\mathrm{pCi} / \mathrm{L}$ \\
\hline 06 Nov 91 & 14.5 & 5.0 & & $\mathrm{mg} / \mathrm{L}$ & 91.90 & 129.74 & $\mathrm{pCi} / \mathrm{g}$ \\
\hline 07 Nov 91 & 20.8 & & & & 0.62 & 0.86 & $\mathrm{pCi} / \mathrm{L}$ \\
\hline 11 Nov 91 & 20.8 & & & & 1.11 & 0.84 & $\mathrm{pCi} / \mathrm{L}$ \\
\hline 11 Nov 91 & 20.8 & 5.0 & & $\mathrm{mg} / \mathrm{L}$ & 221.65 & 167.59 & $\mathrm{pCi} / \mathrm{g}$ \\
\hline 15 Nov 91 & 20.8 & & & & 0.46 & 1.30 & $\mathrm{pCi} / \mathrm{L}$ \\
\hline 15 Nov 91 & 20.8 & 7.0 & & $\mathbf{m g} / \mathrm{L}$ & 65.64 & 185.35 & $\mathrm{pCi} / \mathrm{g}$ \\
\hline 18 Nov 91 & 20.8 & & & & -2.70 & 54.06 & $\mathrm{pCi} / \mathrm{L}$ \\
\hline 18 Nov 91 & 20.8 & 5.0 & & $\mathrm{mg} / \mathrm{L}$ & -540.60 & 10812.00 & $\mathrm{pCi} / \mathrm{g}$ \\
\hline 19 Nov 91 & 20.8 & & & & 1.30 & 2.00 & $\mathrm{pCi} / \mathrm{L}$ \\
\hline 19 Nov 91 & 20.8 & 5.0 & & $\mathbf{m g} / \mathbf{L}$ & 259.49 & 400.04 & $\mathrm{pCi} / \mathrm{g}$ \\
\hline 19 Nov 91 & 14.5 & & & & -0.22 & 1.38 & $\mathrm{pCi} / \mathrm{L}$ \\
\hline 19 Nov 91 & 14.5 & 5.0 & & $\mathrm{mg} / \mathrm{L}$ & -43.25 & 275.71 & $\mathrm{pCi} / \mathrm{g}$ \\
\hline 20 Nov 91 & 20.8 & & & & 3.24 & 0.81 & $\mathrm{pCi} / \mathrm{L}$ \\
\hline 20 Nov 91 & 20.8 & 5.0 & & $\mathrm{mg} / \mathrm{L}$ & 648.72 & 162.18 & $\mathrm{pCi} / \mathrm{g}$ \\
\hline $02 \operatorname{Dec} 91$ & 20.8 & & & & 27.03 & 5.41 & $\mathrm{pCi} / \mathrm{L}$ \\
\hline $02 \operatorname{Dec} 91$ & 20.8 & 37.0 & 37.0 & $\mathrm{mg} / \mathrm{L}$ & 730.54 & 146.11 & $\mathrm{pCi} / \mathrm{g}$ \\
\hline $02 \operatorname{Dec} 91$ & 14.5 & & & & 0.05 & 1.24 & $\mathrm{pCi} / \mathrm{L}$ \\
\hline $02 \operatorname{Dec} 91$ & 14.5 & 6.0 & 6.0 & $\mathrm{mg} / \mathrm{L}$ & 9.01 & 207.23 & $\mathrm{pCi} / \mathrm{g}$ \\
\hline 03 Dec 91 & 20.8 & & & & 24.33 & 2.97 & $\mathrm{pCi} / \mathrm{L}$ \\
\hline
\end{tabular}


Table A.3 (continued)

\begin{tabular}{|c|c|c|c|c|c|c|c|}
\hline Date & $\begin{array}{c}\text { River } \\
\text { mile }\end{array}$ & $\begin{array}{l}\text { TSP } \\
\text { result }\end{array}$ & Error & Units & $\begin{array}{l}{ }^{137} \mathrm{Cs} \\
\text { result }\end{array}$ & $\begin{array}{l}\text { Rad } \\
\text { error }\end{array}$ & Unit \\
\hline 03 Dec 91 & 20.8 & 24.0 & 24.0 & $\mathrm{mg} / \mathrm{L}$ & 1013.63 & 123.89 & $\mathrm{pCi} / \mathrm{g}$ \\
\hline $03 \operatorname{Dec} 91$ & 14.5 & & & & 1.38 & 1.62 & $\mathrm{pCi} / \mathrm{L}$ \\
\hline $03 \operatorname{Dec} 91$ & 14.5 & 11.0 & 11.0 & $\mathrm{mg} / \mathrm{L}$ & 125.32 & 147.44 & $\mathrm{pCi} / \mathrm{g}$ \\
\hline $04 \operatorname{Dec} 91$ & 20.8 & & & & 7.30 & 2.97 & $\mathrm{pCi} / \mathrm{L}$ \\
\hline $04 \operatorname{Dec} 91$ & 20.8 & 13.0 & 13.0 & $\mathrm{mg} / \mathrm{L}$ & 561.39 & 228.72 & $\mathrm{pCi} / \mathrm{g}$ \\
\hline 04 Dec 91 & 14.5 & & & & 0.22 & 2.68 & $\mathrm{pCi} / \mathrm{L}$ \\
\hline $04 \operatorname{Dec} 91$ & 14.5 & 10.0 & 10.0 & $\mathrm{mg} / \mathrm{L}$ & 21.62 & 267.60 & $\mathrm{pCi} / \mathrm{g}$ \\
\hline 05 Dec 91 & 20.8 & & & & 2.70 & 1.35 & $\mathrm{pCi} / \mathrm{L}$ \\
\hline 05 Dec 91 & 20.8 & 12.0 & 12.0 & $\mathrm{mg} / \mathrm{L}$ & 225.25 & 112.63 & $\mathrm{pCi} / \mathrm{g}$ \\
\hline 05 Dec 91 & 14.5 & & & & 0.43 & 1.03 & $\mathrm{pCi} / \mathrm{L}$ \\
\hline $05 \operatorname{Dec} 91$ & 14.5 & 16.0 & 16.0 & $\mathrm{mg} / \mathrm{L}$ & 27.03 & 64.20 & $\mathrm{pCi} / \mathrm{g}$ \\
\hline $06 \operatorname{Dec} 91$ & 20.8 & & & & 0.54 & 2.70 & $\mathrm{pCi} / \mathrm{L}$ \\
\hline 06 Dec 91 & 20.8 & 30.0 & & $\mathrm{mg} / \mathrm{L}$ & 18.02 & 90.10 & $\mathrm{pCi} / \mathrm{g}$ \\
\hline 06 Dec 91 & 14.5 & & & & 1.54 & 2.46 & $\mathrm{pCi} / \mathrm{L}$ \\
\hline O6 Dec 91 & 14.5 & 21.0 & 21.0 & $\mathrm{mg} / \mathrm{L}$ & 73.37 & 117.13 & $\mathrm{pCi} / \mathrm{g}$ \\
\hline $09 \operatorname{Dec} 91$ & 14.5 & & & & 0.92 & 1.41 & $\mathrm{pCi} / \mathrm{L}$ \\
\hline 09 Dec 91 & 14.5 & 8.0 & 8.0 & $\mathrm{mg} / \mathrm{L}$ & 114.88 & 175.70 & $\mathrm{pCi} / \mathrm{g}$ \\
\hline 10 Dec 91 & 14.5 & & & & 1.05 & 0.62 & $\mathrm{pCi} / \mathrm{L}$ \\
\hline 10 Dec 91 & 14.5 & 6.0 & 6.0 & $\mathrm{mg} / \mathrm{L}$ & 175.70 & 103.62 & $\mathrm{pCi} / \mathrm{g}$ \\
\hline $12 \operatorname{Dec} 91$ & 14.5 & & & & 0.30 & 0.81 & $\mathrm{pCi} / \mathrm{L}$ \\
\hline $12 \operatorname{Dec} 91$ & 14.5 & 6.0 & & $\mathrm{mg} / \mathrm{L}$ & 49.56 & 135.15 & $\mathrm{pCi} / \mathrm{g}$ \\
\hline $13 \operatorname{Dec} 91$ & 14.5 & & & & 0.30 & 1.73 & $\mathrm{pCi} / \mathrm{L}$ \\
\hline 13 Dec 91 & 14.5 & 7.0 & & $\mathrm{mg} / \mathrm{L}$ & 42.48 & 247.13 & pCi/g \\
\hline $16 \operatorname{Dec} 91$ & 14.5 & & & & -0.05 & 1.19 & $\mathrm{pCi} / \mathrm{L}$ \\
\hline 16 Dec 91 & 14.5 & 5.0 & & $\mathrm{mg} / \mathrm{L}$ & -10.81 & 237.86 & $\mathrm{pCi} / \mathrm{g}$ \\
\hline 17 Dec 91 & 20.8 & & & & -0.32 & 2.30 & $\mathrm{pCi} / \mathrm{L}$ \\
\hline $17 \operatorname{Dec} 91$ & 20.8 & 10.0 & & $\mathrm{mg} / \mathrm{L}$ & -32.44 & 229.76 & $\mathrm{pCi} / \mathrm{g}$ \\
\hline 17 Dec 91 & 14.5 & & & & 0.11 & 1.24 & $\mathrm{pCi} / \mathrm{L}$ \\
\hline 17 Dec 91 & 14.5 & 7.0 & & $\mathrm{mg} / \mathrm{L}$ & 15.45 & 177.63 & $\mathrm{pCi} / \mathrm{g}$ \\
\hline 18 Dec 91 & 14.5 & & & & 0.32 & 1.00 & $\mathrm{pCi} / \mathrm{L}$ \\
\hline $18 \operatorname{Dec} 91$ & 14.5 & 53.0 & & $\mathrm{mg} / \mathrm{L}$ & 6.12 & 18.87 & $\mathrm{pCi} / \mathrm{g}$ \\
\hline
\end{tabular}




\section{A-26}

Table A.3 (continued)

\begin{tabular}{|c|c|c|c|c|c|c|c|}
\hline Date & $\begin{array}{l}\text { River } \\
\text { mile }\end{array}$ & $\begin{array}{l}\text { TSP } \\
\text { result }\end{array}$ & Error & Units & $\begin{array}{l}{ }^{237} \mathrm{Cs} \\
\text { result }\end{array}$ & $\begin{array}{l}\text { Rad } \\
\text { error }\end{array}$ & Unit \\
\hline $19 \operatorname{Dec} 91$ & 14.5 & & & & 0.24 & 0.89 & $\mathrm{pCi} / \mathrm{L}$ \\
\hline $19 \operatorname{Dec} 91$ & 14.5 & 8.0 & & $\mathrm{mg} / \mathrm{L}$ & 30.41 & 111.50 & $\mathrm{pCi} / \mathrm{g}$ \\
\hline 20 Dec 91 & 14.5 & & & & 1.08 & 1.08 & $\mathrm{pCi} / \mathrm{L}$ \\
\hline 20 Dec 91 & 14.5 & 25.0 & & $\mathrm{mg} / \mathrm{L}$ & 43.25 & 43.25 & $\mathrm{pCi} / \mathrm{g}$ \\
\hline 26 Dec 91 & 20.8 & & & & 0.24 & 1.41 & $\mathrm{pCi} / \mathrm{L}$ \\
\hline $26 \operatorname{Dec} 91$ & 20.8 & 8.0 & & $\mathrm{mg} / \mathrm{L}$ & 30.41 & 175.70 & $\mathrm{pCi} / \mathrm{g}$ \\
\hline 26 Dec 91 & 14.5 & & & & 0.08 & 1.35 & $\mathrm{pCi} / \mathrm{L}$ \\
\hline $26 \operatorname{Dec} 91$ & 14.5 & 6.0 & & $\mathrm{mg} / \mathrm{L}$ & 13.52 & 225.25 & $\mathrm{pCi} / \mathrm{g}$ \\
\hline 30 Dec 91 & 20.8 & & & & 2.51 & 1.78 & $\mathrm{pCi} / \mathrm{L}$ \\
\hline $30 \operatorname{Dec} 91$ & 20.8 & 51.0 & & $\mathrm{mg} / \mathrm{L}$ & 49.29 & 34.98 & $\mathrm{pCi} / \mathrm{g}$ \\
\hline 30 Dec 91 & 14.5 & & & & 1.43 & 1.19 & $\mathrm{pCi} / \mathrm{L}$ \\
\hline 30 Dec 91 & 14.5 & 49.0 & & $\mathrm{mg} / \mathrm{L}$ & 29.24 & 24.27 & $\mathrm{pCi} / \mathrm{g}$ \\
\hline $31 \operatorname{Dec} 91$ & 14.5 & & & & 1.03 & 0.97 & $\mathrm{pCi} / \mathrm{L}$ \\
\hline $31 \operatorname{Dec} 91$ & 14.5 & 36.0 & & $m g / L$ & 28.53 & 27.03 & $\mathrm{pCi} / \mathrm{g}$ \\
\hline $02 \mathrm{Jan} 92$ & 14.5 & & & & 0.49 & 0.54 & $\mathrm{pCi} / \mathrm{L}$ \\
\hline $02 \operatorname{Jan} 92$ & 14.5 & 30.0 & & $\mathrm{mg} / \mathrm{L}$ & 16.22 & 18.02 & $\mathrm{pCi} / \mathrm{g}$ \\
\hline $03 \mathrm{Jan} 92$ & 14.5 & & & & 0.46 & 0.57 & $\mathrm{pCi} / \mathrm{L}$ \\
\hline $03 \mathrm{Jan} 92$ & 14.5 & 12.0 & & $\mathrm{mg} / \mathrm{L}$ & 38.29 & 47.30 & $\mathrm{pCi} / \mathrm{g}$ \\
\hline 06 Jan 92 & 20.8 & & & & 1.00 & 2.11 & $\mathrm{pCi} / \mathrm{L}$ \\
\hline 06 Jan 92 & 20.8 & 8.0 & & $\mathrm{mg} / \mathrm{L}$ & 125.01 & 263.54 & $\mathrm{pCi} / \mathrm{g}$ \\
\hline $06 \operatorname{Jan} 92$ & 14.5 & & & & 0.89 & 1.03 & pCill \\
\hline $06 \operatorname{Jan} 92$ & 14.5 & 36.0 & & $\mathrm{mg} / \mathrm{L}$ & 24.78 & 28.53 & $\mathrm{pCi} / \mathrm{g}$ \\
\hline 07 Jan 92 & 14.5 & & & & 0.97 & 0.84 & $\mathrm{pCi} / \mathrm{L}$ \\
\hline 07 Jan 92 & 14.5 & 41.0 & & $\mathrm{mg} / \mathrm{L}$ & 23.73 & 20.44 & $\mathrm{pCi} / \mathrm{g}$ \\
\hline $09 \operatorname{Jan} 92$ & 14.5 & & & & 1.05 & 1.19 & $\mathrm{pCi} / \mathrm{L}$ \\
\hline $09 \operatorname{Jan} 92$ & 14.5 & 30.0 & & $\mathrm{mg} / \mathrm{L}$ & 35.14 & 39.64 & $\mathrm{pCi} / \mathrm{g}$ \\
\hline 13 Jan 92 & 14.5 & & & & 2.00 & 1.19 & $\mathrm{pCi} / \mathrm{L}$ \\
\hline $13 \mathrm{Jan} 92$ & 14.5 & 288.0 & & $\mathrm{mg} / \mathrm{L}$ & 6.95 & 4.13 & $\mathrm{pCi} / \mathrm{g}$ \\
\hline $14 \operatorname{Jan} 92$ & 14.5 & & & & 2.38 & 0.59 & $\mathrm{pCi} / \mathrm{L}$ \\
\hline $14 \operatorname{Jan} 92$ & 14.5 & 131.0 & & $\mathrm{mg} / \mathrm{L}$ & 18.16 & 4.54 & $\mathrm{pCi} / \mathrm{g}$ \\
\hline 15 Jan 92 & 14.5 & & & & 0.65 & 1.32 & $\mathrm{pCi} / \mathrm{L}$ \\
\hline
\end{tabular}


Table A.3 (continued)

\begin{tabular}{|c|c|c|c|c|c|c|c|}
\hline Date & $\begin{array}{c}\text { River } \\
\text { mile }\end{array}$ & $\begin{array}{c}\text { TSP } \\
\text { result }\end{array}$ & Error & Units & $\begin{array}{l}{ }^{137} \mathrm{Cs} \\
\text { result }\end{array}$ & $\begin{array}{l}\text { Rad } \\
\text { error }\end{array}$ & Unit \\
\hline $15 \operatorname{Jan} 92$ & 14.5 & 12.0 & & $\mathrm{mg} / \mathrm{L}$ & 54.06 & 110.37 & $\mathrm{pCi} / \mathrm{g}$ \\
\hline $16 \operatorname{Jan} 92$ & 14.5 & & & & 1.35 & 1.27 & $\mathrm{pCi} / \mathrm{L}$ \\
\hline $16 \operatorname{Jan} 92$ & 14.5 & 80.0 & & $\mathrm{mg} / \mathrm{L}$ & 16.89 & 15.88 & $\mathrm{pCi} / \mathrm{g}$ \\
\hline $17 \operatorname{Jan} 92$ & 14.5 & & & & 0.30 & 1.00 & $\mathrm{pCi} / \mathrm{L}$ \\
\hline $17 \operatorname{Jan} 92$ & 14.5 & 10.0 & & $\mathrm{mg} / \mathrm{L}$ & 29.73 & 100.01 & $\mathrm{pCi} / \mathrm{g}$ \\
\hline $20 \operatorname{Jan} 92$ & 14.5 & & & & 2.97 & 0.81 & $\mathrm{pCi} / \mathrm{L}$ \\
\hline $20 \operatorname{Jan} 92$ & 14.5 & 88.0 & & $\mathrm{mg} / \mathrm{L}$ & 33.79 & 9.21 & $\mathrm{pCi} / \mathrm{g}$ \\
\hline $21 \operatorname{Jan} 92$ & 14.5 & & & & 0.57 & 1.30 & $\mathrm{pCi} / \mathrm{L}$ \\
\hline $21 \operatorname{Jan} 92$ & 20.8 & 28.0 & & $\mathrm{mg} / \mathrm{L}$ & 20.27 & 46.34 & $\mathrm{pCi} / \mathrm{g}$ \\
\hline $22 \operatorname{Jan} 92$ & 14.5 & & & & 0.46 & 0.84 & $\mathrm{pCi} / \mathrm{L}$ \\
\hline $22 \operatorname{Jan} 92$ & 14.5 & 5.0 & & $\mathrm{mg} / \mathrm{L}$ & 91.90 & 167.59 & $\mathrm{pCi} / \mathrm{g}$ \\
\hline $23 \operatorname{Jan} 92$ & 14.5 & & & & 0.68 & 0.76 & $\mathrm{pCi} / \mathrm{L}$ \\
\hline $23 \operatorname{Jan} 92$ & 14.5 & 20.0 & & $\mathrm{mg} / \mathrm{L}$ & 33.79 & 37.84 & $\mathrm{pCi} / \mathrm{g}$ \\
\hline $24 \operatorname{Jan} 92$ & 14.5 & & & & 0.08 & 0.92 & $\mathrm{pCi} / \mathrm{L}$ \\
\hline $24 \operatorname{Jan} 92$ & 14.5 & 7.0 & & $\mathrm{mg} / \mathrm{L}$ & 11.58 & 131.29 & $\mathrm{pCi} / \mathrm{g}$ \\
\hline 27 Jan 92 & 14.5 & & & & 0.92 & 0.59 & $\mathrm{pCi} / \mathrm{L}$ \\
\hline 27 Jan 92 & 14.5 & 27.0 & & $\mathrm{mg} / \mathrm{L}$ & 34.04 & 22.02 & $\mathrm{pCi} / \mathrm{g}$ \\
\hline 28 Jan 92 & 14.5 & & & & 0.78 & 0.54 & $\mathrm{pCi} / \mathrm{L}$ \\
\hline $28 \operatorname{Jan} 92$ & 14.5 & 36.0 & & $\mathrm{mg} / \mathrm{L}$ & 21.77 & 15.02 & $\mathrm{pCi} / \mathrm{g}$ \\
\hline $29 \operatorname{Jan} 92$ & 14.5 & & & & 0.14 & 0.76 & $\mathrm{pCi} / \mathrm{L}$ \\
\hline $29 \operatorname{Ian} 92$ & 14.5 & 5.0 & & $\mathrm{mg} / \mathrm{L}$ & 27.03 & 151.37 & $\mathrm{pCi} / \mathrm{g}$ \\
\hline $30 \mathrm{~J}$ 结螎 92 & 14.5 & & & & 0.30 & 0.62 & $\mathrm{pCi} / \mathrm{L}$ \\
\hline 30 Jan 92 & 14.5 & 5.0 & & $\mathrm{mg} / \mathrm{L}$ & 59.47 & 124.34 & $\mathrm{pCi} / \mathrm{g}$ \\
\hline 05 Feb 92 & 20.8 & & & & 5.14 & 1.08 & $\mathrm{pCi} / \mathrm{L}$ \\
\hline 05 Feb 92 & 20.8 & 7.0 & & $\mathrm{mg} / \mathrm{L}$ & 733.67 & 154.46 & $\mathrm{pCi} / \mathrm{g}$ \\
\hline 06 Feb 92 & 20.8 & & & & 2.57 & 0.78 & $\mathrm{pCi} / \mathrm{L}$ \\
\hline 06 Feb 92 & 20.8 & 7.0 & & $\mathrm{mg} / \mathrm{L}$ & 366.84 & 111.98 & $\mathrm{pCi} / \mathrm{g}$ \\
\hline 07 Feb 92 & 20.8 & & & & 2.57 & 1.87 & $\mathrm{pCi} / \mathrm{L}$ \\
\hline 07 Feb 92 & 20.8 & 8.0 & & $\mathrm{mg} / \mathrm{L}$ & 320.98 & 233.13 & $\mathrm{pCi} / \mathrm{g}$ \\
\hline 12 Feb 92 & 20.8 & & & & 7.30 & 1.35 & $\mathrm{pCi} / \mathrm{L}$ \\
\hline 12 Feb 92 & 20.8 & 7.0 & & $\mathrm{mg} / \mathrm{L}$ & 1042.59 & 193.07 & $\mathrm{pCi} / \mathrm{g}$ \\
\hline
\end{tabular}


Table A.3 (continued)

\begin{tabular}{|c|c|c|c|c|c|c|c|}
\hline Date & $\begin{array}{c}\text { River } \\
\text { mile }\end{array}$ & $\begin{array}{l}\text { TSP } \\
\text { result }\end{array}$ & Error & Units & $\begin{array}{l}{ }^{137} \mathrm{Cs} \\
\text { result }\end{array}$ & $\begin{array}{l}\mathrm{Rad} \\
\text { error }\end{array}$ & Unit \\
\hline 18 Feb 92 & 20.8 & & & & 2.97 & 1.35 & $\mathrm{pCi} / \mathrm{L}$ \\
\hline 18 Feb 92 & 20.8 & 5.0 & & $\mathrm{mg} / \mathrm{L}$ & 594.66 & 270.30 & $\mathrm{pCi} / \mathrm{g}$ \\
\hline 19 Feb 92 & 20.8 & & & & 14.33 & 1.89 & $\mathrm{pCi} / \mathrm{L}$ \\
\hline 19 Feb 92 & 20.8 & 10.0 & & $\mathrm{mg} / \mathrm{L}$ & 1432.59 & 189.21 & $\mathrm{pCi} / \mathrm{g}$ \\
\hline 21 Feb 92 & 20.8 & & & & 16.49 & 2.16 & $\mathrm{pCi} / \mathrm{L}$ \\
\hline 21 Feb 92 & 20.8 & 8.0 & & $\mathrm{mg} / \mathrm{L}$ & 2061.04 & 270.30 & $\mathrm{pCi} / \mathrm{g}$ \\
\hline 25 Feb 92 & 20.8 & & & & 43.25 & 5.41 & $\mathrm{pCi} / \mathrm{L}$ \\
\hline 25 Feb 92 & 20.8 & 14.0 & & $\mathrm{mg} / \mathrm{L}$ & 3089.14 & 386.14 & $\mathrm{pCi} / \mathrm{g}$ \\
\hline 26 Feb 92 & 20.8 & & & & 32.44 & 2.70 & $\mathrm{pCi} / \mathrm{L}$ \\
\hline 26 Feb 92 & 20.8 & 16.0 & & $\mathrm{mg} / \mathrm{L}$ & 2027.25 & 168.94 & pCi/g \\
\hline 27 Feb 92 & 20.8 & & & & 26.22 & 4.32 & $\mathrm{pCi} / \mathrm{L}$ \\
\hline 27 Feb 92 & 20.8 & 17.0 & & $\mathrm{mg} / \mathrm{L}$ & 1542.30 & 254.40 & $\mathrm{pCi} / \mathrm{g}$ \\
\hline 03 Mar 92 & 20.8 & & & & 20.54 & 1.89 & $\mathrm{pCi} / \mathrm{L}$ \\
\hline 03 Mar 92 & 20.8 & 11.0 & & $\mathrm{mg} / \mathrm{L}$ & 1867.53 & 172.01 & $\mathrm{pCi} / \mathrm{g}$ \\
\hline 05 Mar 92 & 20.8 & & & & 26.49 & 2.43 & $\mathrm{pCi} / \mathrm{L}$ \\
\hline $05 \operatorname{Mar} 92$ & 20.8 & 8.0 & & $\mathrm{mg} / \mathrm{L}$ & 3311.18 & 304.09 & $\mathrm{pCi} / \mathrm{g}$ \\
\hline 06 Mar 92 & 20.8 & & & & 20.27 & 1.62 & $\mathrm{pCi} / \mathrm{L}$ \\
\hline 06 Mar 92 & 20.8 & 10.0 & & $\mathrm{mg} / \mathrm{L}$ & 2027.25 & 162.18 & $\mathrm{pCi} / \mathrm{g}$ \\
\hline 10 Mar 92 & 20.8 & & & & 23.25 & 1.89 & $\mathrm{pCi} / \mathrm{L}$ \\
\hline $10 \operatorname{Mar} 92$ & 20.8 & 13.0 & & $\mathrm{mg} / \mathrm{L}$ & 1788.14 & 145.55 & $\mathrm{pCi} / \mathrm{g}$ \\
\hline 18 Mar 92 & 20.8 & & & & 16.49 & 1.62 & $\mathrm{pCi} / \mathrm{L}$ \\
\hline $18 \operatorname{Mar} 92$ & 20.8 & 14.0 & & $\mathrm{mg} / \mathrm{L}$ & 1177.74 & 115.84 & $\mathrm{pCi} / \mathrm{g}$ \\
\hline $19 \operatorname{Mar} 92$ & 20.8 & & & & 32.44 & 2.70 & $\mathrm{pCi} / \mathrm{L}$ \\
\hline $19 \operatorname{Mar} 92$ & 20.8 & 19.0 & & $\mathrm{mg} / \mathrm{L}$ & 1707.16 & 142.26 & $\mathrm{pCi} / \mathrm{g}$ \\
\hline 20 Mar 92 & 20.8 & & & & 43.25 & 2.70 & $\mathrm{pCi} / \mathrm{L}$ \\
\hline 20 Mar 92 & 20.8 & 19.0 & & $\mathrm{mg} / \mathrm{L}$ & 2276.21 & 142.26 & $\mathrm{pCi} / \mathrm{g}$ \\
\hline $24 \operatorname{Mar} 92$ & 20.8 & & & & 25.68 & 2.16 & $\mathrm{pCi} / \mathrm{L}$ \\
\hline $24 \operatorname{Mar} 92$ & 20.8 & 10.0 & & $\mathrm{mg} / \mathrm{L}$ & 2567.85 & 216.24 & $\mathrm{pCi} / \mathrm{g}$ \\
\hline 25 Mar 92 & 20.8 & & & & 19.46 & 1.62 & $\mathrm{pCi} / \mathrm{L}$ \\
\hline $25 \operatorname{Mar} 92$ & 20.8 & 11.0 & & $\mathrm{mg} / \mathrm{L}$ & 1769.24 & 147.44 & $\mathrm{pCi} / \mathrm{g}$ \\
\hline $26 \operatorname{Mar} 92$ & 20.8 & & & & 17.30 & 1.62 & $\mathrm{pCi} / \mathrm{L}$ \\
\hline
\end{tabular}


Table A.3 (continued)

\begin{tabular}{|c|c|c|c|c|c|c|c|}
\hline Date & $\begin{array}{c}\text { River } \\
\text { mile }\end{array}$ & $\begin{array}{l}\text { TSP } \\
\text { result }\end{array}$ & Error & Units & $\begin{array}{l}{ }^{137} \mathrm{Cs} \\
\text { result }\end{array}$ & $\begin{array}{l}\text { Rad } \\
\text { error }\end{array}$ & Unit \\
\hline $26 \operatorname{Mar} 92$ & 20.8 & 24.0 & & $\mathrm{mg} / \mathrm{L}$ & 720.80 & 67.58 & $\mathrm{pCi} / \mathrm{g}$ \\
\hline $26 \operatorname{Mar} 92$ & 14.5 & & & & 1.27 & 0.78 & $\mathrm{pCi} / \mathrm{L}$ \\
\hline $26 \operatorname{Mar} 92$ & 14.5 & 42.0 & & $\mathrm{mg} / \mathrm{L}$ & 30.25 & 18.66 & $\mathrm{pCi} / \mathrm{g}$ \\
\hline $27 \operatorname{Mar} 92$ & 20.8 & & & & 62.17 & 2.70 & $\mathrm{pCi} / \mathrm{L}$ \\
\hline 27 Mar 92 & 20.8 & 33.0 & & $\mathrm{mg} / \mathrm{L}$ & 1883.91 & 81.91 & $\mathrm{pCi} / \mathrm{g}$ \\
\hline $31 \operatorname{Mar} 92$ & 20.8 & & & & 29.73 & 2.70 & $\mathrm{pCi} / \mathrm{L}$ \\
\hline $31 \operatorname{Mar} 92$ & 20.8 & 11.0 & & $\mathbf{m g} / \mathrm{L}$ & 2703.00 & 245.73 & $\mathrm{pCi} / \mathrm{g}$ \\
\hline 01 Apr 92 & 20.8 & & & & 1.97 & 0.95 & $\mathrm{pCi} / \mathrm{L}$ \\
\hline 01 Apr 92 & 20.8 & 5.0 & & $\mathrm{mg} / \mathrm{L}$ & 394.64 & 189.21 & $\mathrm{pCi} / \mathrm{g}$ \\
\hline 02 Apr 92 & 20.8 & & & & 37.84 & 2.70 & $\mathrm{pCi} / \mathrm{L}$ \\
\hline 02 Apr 92 & 20.8 & 13.0 & & $\mathrm{mg} / \mathrm{L}$ & 2910.92 & 207.92 & $\mathrm{pCi} / \mathrm{g}$ \\
\hline 02 Apr 92 & 14.5 & & & & 1.57 & 0.95 & $\mathrm{pCi} / \mathrm{L}$ \\
\hline 02 Apr 92 & 14.5 & 7.0 & & $\mathrm{mg} / \mathrm{L}$ & 223.96 & 135.15 & $\mathrm{pCi} / \mathrm{g}$ \\
\hline 07 Apr 92 & 20.8 & & & & 25.41 & 2.16 & $\mathrm{pCi} / \mathrm{L}$ \\
\hline 07 Apr 92 & 20.8 & 10.0 & & $\mathrm{mg} / \mathrm{L}$ & 2540.82 & 216.24 & $\mathrm{pCi} / \mathrm{g}$ \\
\hline 08 Apr 92 & 20.8 & & & & 27.03 & 2.70 & $\mathrm{pCi} / \mathrm{L}$ \\
\hline 08 Apr 92 & 20.8 & 14.0 & & $\mathrm{mg} / \mathrm{L}$ & 1930.71 & 193.07 & $\mathrm{pCi} / \mathrm{g}$ \\
\hline 08 Apr 92 & 14.5 & & & & 2.97 & 1.08 & $\mathrm{pCi} / \mathrm{L}$ \\
\hline 08 Apr 92 & 14.5 & 12.0 & & $\mathrm{mg} / \mathrm{L}$ & 247.78 & 90.10 & $\mathrm{pCi} / \mathrm{g}$ \\
\hline 09 Apr 92 & 20.8 & & & & 24.60 & 1.89 & $\mathrm{pCi} / \mathrm{L}$ \\
\hline 09 Apr 92 & 20.8 & 5.0 & & $\mathrm{mg} / \mathrm{L}$ & 4919.46 & 378.42 & $\mathrm{pCi} / \mathrm{g}$ \\
\hline 09 Apr 92 & 14.5 & & & & 2.43 & 4.05 & pCilL \\
\hline 09 Apr 92 & 14.5 & 32.0 & & $\mathrm{mg} / \mathrm{L}$ & 76.02 & 126.70 & pCiig \\
\hline 10 Apr 92 & 20.8 & & & & 32.44 & 2.70 & $\mathrm{pCi} / \mathrm{L}$ \\
\hline 10 Apr 92 & 20.8 & 11.0 & & $\mathrm{mg} / \mathrm{L}$ & 2948.73 & 245.73 & $\mathrm{pCi} / \mathrm{g}$ \\
\hline 10 Apr 92 & 14.5 & & & & 1.68 & 0.65 & $\mathrm{pCi} / \mathrm{L}$ \\
\hline 10 Apr 92 & 14.5 & 12.0 & & $\mathrm{mg} / \mathrm{L}$ & 139.66 & 54.06 & $\mathrm{pCi} / \mathrm{g}$ \\
\hline 16 Apr 92 & 20.8 & & & & 18.11 & 1.62 & $\mathrm{pCi} / \mathrm{L}$ \\
\hline 16 Apr 92 & 20.8 & 12.0 & 12.0 & $\mathrm{mg} / \mathrm{L}$ & 1509.18 & 135.15 & $\mathrm{pCi} / \mathrm{g}$ \\
\hline
\end{tabular}


Appendix B

ROUTINE WATER QUALITY AND CONTAMINANT RESULTS FROM THE WHITE OAK DAM MONITORING STATION 
Table B.1. Radiological data for White Oak Dam outflows, June 1991 though June 1992 Data provided by ORNL Environmental Monitoring and Compliance.

\begin{tabular}{|c|c|c|c|c|c|c|c|}
\hline SAMP DATE & $\begin{array}{l}{ }^{\infty} \mathrm{Co} \\
\mathrm{pCi} / \mathrm{L}\end{array}$ & $\begin{array}{l}{ }^{\infty} \mathrm{Co} \\
\mathrm{er}\end{array}$ & $\begin{array}{l}{ }^{10} \mathrm{Cs} \\
\mathrm{pCi} / \mathrm{L}\end{array}$ & $\begin{array}{l}{ }^{19} \mathrm{Cs} \\
\text { err }\end{array}$ & $\begin{array}{c}\text { Gross alpha } \\
\text { pCi/L }\end{array}$ & $\begin{array}{l}\text { Alpha } \\
\text { ert }\end{array}$ & $\begin{array}{l}\text { Gross beta } \\
\mathrm{pCi} / \mathrm{L}\end{array}$ \\
\hline 05 Jun 91 & 5.14 & 2.97 & 26.76 & 3.24 & 5.95 & 4.05 & 378.42 \\
\hline 12 Jun 91 & -0.54 & 13.79 & 78.39 & 10.81 & 32.44 & 8.11 & 486.54 \\
\hline 19 Jun 91 & 4.32 & 6.22 & 43.25 & 5.41 & 8.11 & 4.60 & 351.39 \\
\hline 26 Jun 91 & 8.92 & 7.03 & 105.42 & 13.52 & 4.60 & 2.70 & 378.42 \\
\hline 03 Jul 91 & -0.27 & 6.49 & 20.27 & 3.24 & 6.22 & 2.97 & 405.45 \\
\hline $10 \mathrm{Ju!} 91$ & 0.81 & 6.49 & 37.84 & 5.41 & 4.87 & 2.43 & 405.45 \\
\hline 17 Jul 91 & 2.70 & 40.55 & 513.57 & 54.06 & 6.49 & 3.78 & 459.51 \\
\hline 24 Jul 91 & 10.00 & 9.46 & 64.87 & 13.52 & 9.19 & 3.78 & 486.54 \\
\hline 31 Jul 91 & -2.70 & 16.22 & 37.84 & 13.52 & 15.14 & 5.14 & 1135.26 \\
\hline 07 Aug 91 & 6.76 & 10.54 & 24.33 & 10.27 & 6.76 & 2.97 & 351.39 \\
\hline 14 Aus 91 & 7.84 & 10.54 & 78.39 & 13.52 & 3.51 & 2.43 & 43248 \\
\hline 21 Aue 91 & 2.70 & 7.30 & 124.34 & 8.11 & 4.60 & 2.70 & 297.33 \\
\hline 28 Aug 91 & 4.87 & 2.97 & 89.20 & 5.41 & 7.03 & 3.24 & 224.35 \\
\hline 04 Sep 91 & 0.81 & 10.00 & 29.73 & 10.81 & 3.78 & 2.43 & 205.43 \\
\hline 11 Sep 91 & -2.97 & 6.22 & 40.55 & 5.41 & 5.14 & 2.97 & 267.60 \\
\hline 18 Sep 91 & 4.32 & 243 & 94.60 & 5.41 & 5.14 & 3.78 & 540.60 \\
\hline 25 Sep 91 & 8.11 & 19.73 & 37.84 & 10.81 & 5.14 & 2.97 & 378.42 \\
\hline 02 Oct 91 & 3.78 & 2.16 & 91.90 & 5.41 & 9.73 & 3.78 & 432.48 \\
\hline 09 Oct 91 & 4.87 & 2.70 & 75.68 & 5.41 & 2.24 & 2.05 & 32436 \\
\hline 16 Oct 91 & 1.89 & 7.84 & 40.55 & 5.41 & 8.11 & 3.51 & 324.36 \\
\hline 23 Oct 91 & 4.60 & 3.51 & 56.76 & 5.41 & 6.49 & 3.24 & 486.54 \\
\hline 30 Oct 91 & 216 & 3.24 & 40.55 & 2.70 & 16.49 & 5.14 & 297.33 \\
\hline
\end{tabular}

\begin{tabular}{|c|c|c|c|c|c|}
\hline SAMP DATE & $\begin{array}{l}\text { Beta } \\
\text { err }\end{array}$ & $\begin{array}{l}\text { Total Sr } \\
\text { pCi/L }\end{array}$ & $\begin{array}{l}\text { Sr } \\
\text { err }\end{array}$ & $\begin{array}{l}\text { 'H } \\
\mathrm{pCi} / \mathrm{L}\end{array}$ & $\begin{array}{l}\text { 'H } \\
\text { ert }\end{array}$ \\
\hline 05 Jun 91 & 27.03 & - & • & $\cdot$ & - \\
\hline 12 Jun 91 & 27.03 & . & - & $\cdot$ & • \\
\hline 19 Jun 91 & 27.03 & $\cdot$ & $\therefore$ & $\cdot$ & 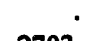 \\
\hline 26 Jun 91 & 27.03 & 202.73 & 9.46 & 137853 & 2703 \\
\hline 03 Jul 91 & 27.03 & · & $\cdot$ & • & • \\
\hline $10 \mathrm{Jul} 91$ & 27.03 & - & $\cdot$ & $\cdot$ & • \\
\hline 17 Jul 91 & 27.03 & - & - & - & - \\
\hline 24 Jul 91 & 27.03 & $\therefore$ & $\cdot$ & • & $\cdot$ \\
\hline 31 Jul 91 & 27.03 & 675.75 & 54.06 & 100011 & 2703 \\
\hline 07 Aug 91 & 27.03 & $\cdot$ & $\cdot$ & • & • \\
\hline 14 Aug 91 & 27.03 & . & . & . & - \\
\hline 21 Aug 91 & 27.03 & $\cdot$ & $\therefore$ & $\cdot$ & \\
\hline 28 Aug 91 & 13.52 & 181.10 & 12.43 & 72981 & 24327 \\
\hline 04 Sep 91 & 13.52 & • & · & • & • \\
\hline 11 Sep 91 & 16.22 & . & - & . & • \\
\hline 18 Sep 91 & 27.03 & • & . & . & • \\
\hline $25 \operatorname{Sep} 91$ & 27.03 & 110.82 & 7.57 & 54060 & 2703 \\
\hline 02 Oct 91 & 27.03 & • & $\cdot$ & . & • \\
\hline 09 Oct 91 & 27.03 & . & - & . & $\cdot$ \\
\hline 16 Oct 91 & 27.03 & - & . & · & • \\
\hline 23 Oct 91 & 27.03 & $\therefore$ & $\therefore$ & 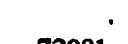 & \\
\hline 30 Oct 91 & 27.03 & 154.07 & 11.62 & 72981 & 2703 \\
\hline
\end{tabular}


Table B.1 (continued)

\begin{tabular}{|c|c|c|c|c|c|c|c|}
\hline SAMP DATE & $\begin{array}{l}{ }^{\infty} \mathrm{Co} \\
\mathrm{pCi} / \mathrm{L}\end{array}$ & $\begin{array}{l}{ }^{\infty} \mathrm{Co} \\
\text { ert }\end{array}$ & $\begin{array}{l}{ }^{19} \mathrm{Cs} \\
\mathrm{pCi} / \mathrm{L}\end{array}$ & $\begin{array}{l}{ }^{17} \mathrm{Cs} \\
\text { err }\end{array}$ & $\begin{array}{c}\text { Gross alpha } \\
\text { pCill }\end{array}$ & $\begin{array}{l}\text { Alphia } \\
\text { ert }\end{array}$ & $\begin{array}{c}\text { Gross beta } \\
\text { pCi/l }\end{array}$ \\
\hline 06 Nov 91 & 5.14 & 2.16 & 86.50 & 5.41 & 8.65 & 3.78 & 297.33 \\
\hline 13 Nov 91 & 0.27 & 4.05 & 27.03 & 2.70 & 5.41 & 2.97 & 243.27 \\
\hline 20 Nov 91 & 4.05 & 7.03 & 48.65 & 2.70 & 4.60 & 2.70 & 270.30 \\
\hline 27 Now 91 & 4.32 & 2.43 & 45.95 & 2.70 & 4.60 & 3.78 & 378.42 \\
\hline 04 Dec 91 & 62.17 & 8.11 & 6.49 & 5.41 & 6.49 & 3.24 & 432.48 \\
\hline 11 Dec 91 & 4.87 & 2.43 & 72.98 & 2.70 & 6.76 & 3.24 & 513.57 \\
\hline 18 Dec 91 & 5.14 & 3.24 & 23.52 & 2.97 & 4.05 & 3.51 & 540.60 \\
\hline 26 Dec 91 & 10.81 & 6.22 & 32.44 & 10.81 & 11.08 & 5.68 & 405.45 \\
\hline $31 \operatorname{Dec} 91$ & 2.97 & 3.24 & 26.49 & 2.97 & 4.05 & 2.70 & 459.51 \\
\hline $08 \mathrm{Jan} 92$ & 6.76 & 7.84 & 35.14 & 8.11 & 13.79 & 6.22 & 486.54 \\
\hline $15 \mathrm{Jan} 92$ & 2.70 & 59.47 & 145.96 & 40.55 & 7.03 & 3.24 & 486.54 \\
\hline 22 Jan 92 & 3.24 & 6.22 & 21.08 & 4.87 & 6.22 & 4.32 & 459.51 \\
\hline $29 \mathrm{Jan} 92$ & 10.27 & 6.76 & 40.55 & 8.11 & 8.92 & 3.78 & 513.57 \\
\hline 05 Feb 92 & 3.78 & 3.78 & 22.98 & 3.78 & 10.54 & 4.05 & 432.48 \\
\hline 12 Feb 92 & 7.57 & 3.78 & 15.14 & 4.05 & 7.57 & 4.60 & 459.51 \\
\hline 19 Feb 92 & 8.11 & 35.14 & 72.98 & 24.33 & 7.03 & 4.32 & 567.63 \\
\hline 26 Feb 92 & 20.81 & 21.62 & 191.91 & 35.14 & 9.19 & 5.14 & 675.75 \\
\hline $04 \operatorname{Mar} 92$ & 1.35 & 5.14 & 40.55 & 5.41 & 13.52 & 5.14 & 513.57 \\
\hline 11 Mar 92 & 8.11 & 27.03 & 154.07 & 32.44 & 5.95 & 4.05 & 405.45 \\
\hline 19 Mar 92 & 11.35 & 7.84 & 113.53 & 13.5? & 9.19 & 3.51 & 486.54 \\
\hline $25 \mathrm{Mar} 92$ & 5.41 & 3.24 & 75.68 & 5.41 & 7.03 & 3.24 & 459.51 \\
\hline 02 Apr 92 & 9.46 & 19.19 & 51.36 & 16.22 & 18.11 & 8.65 & 432.48 \\
\hline
\end{tabular}

\begin{tabular}{|c|c|c|c|c|c|}
\hline SAMP DATE & $\begin{array}{l}\text { Beta } \\
\text { erT }\end{array}$ & $\begin{array}{r}\text { Total } \mathbf{S r} \\
\text { PCi/L }\end{array}$ & $\begin{array}{l}\text { Sr } \\
\text { err }\end{array}$ & $\begin{array}{l}\text { 's } \\
\text { pCi/L }\end{array}$ & $\begin{array}{l}\text { 'H } \\
\text { err }\end{array}$ \\
\hline 06 Now 51 & 27.03 & . & - & - & . \\
\hline 13 Nov 91 & 16.22 & - & . & . & - \\
\hline 20 Nov 91 & 27.03 & . & . & . & . \\
\hline 27 Nov 91 & 27.03 & . & • & • & • \\
\hline 04 Dec 91 & 27.03 & 175.69 & 11.35 & 108120 & 2703 \\
\hline 11 Des 91 & 27.03 & • & $\cdot$ & • & • \\
\hline 18 Dec 91 & 27.03 & - & - & . & - \\
\hline 26 Dec 91 & 27.03 & . & • & • & • \\
\hline $31 \operatorname{Dec} 91$ & 27.03 & 262.19 & 11.35 & 229755 & 2703 \\
\hline $08 \operatorname{Jan} 92$ & 27.03 & . & • & • & • \\
\hline $15 \operatorname{Jan} 92$ & $27.0 ?$ & . & . & - & . \\
\hline $22 \mathrm{Jan} 92$ & 27.03 & $\bullet$ & • & . & . \\
\hline $29 \mathrm{Jan} 92$ & 27.03 & 224.35 & 10.27 & 297330 & 27030 \\
\hline 05 Feb 92 & 27.03 & . & . & . & . \\
\hline 12 Feb 92 & 27.03 & . & . & . & . \\
\hline 19 Feb 92 & 27.03 & . & . & . & • \\
\hline 26 Feb 92 & 27.03 & 324.36 & 19.19 & 351390 & 27030 \\
\hline $04 \operatorname{Mar} 92$ & 27.03 & . & . & • & • \\
\hline $11 \operatorname{Mar} 92$ & 27.03 & . & - & - & . \\
\hline 19 Mar 92 & 27.03 & • & • & • & \\
\hline 25 Mar 92. & 27.03 & 227.05 & 12.43 & 248676 & 2703 \\
\hline 02 Apr 92 & 27.03 & . & • & • & • \\
\hline
\end{tabular}




\section{B-5}

Table B.1 (continued)

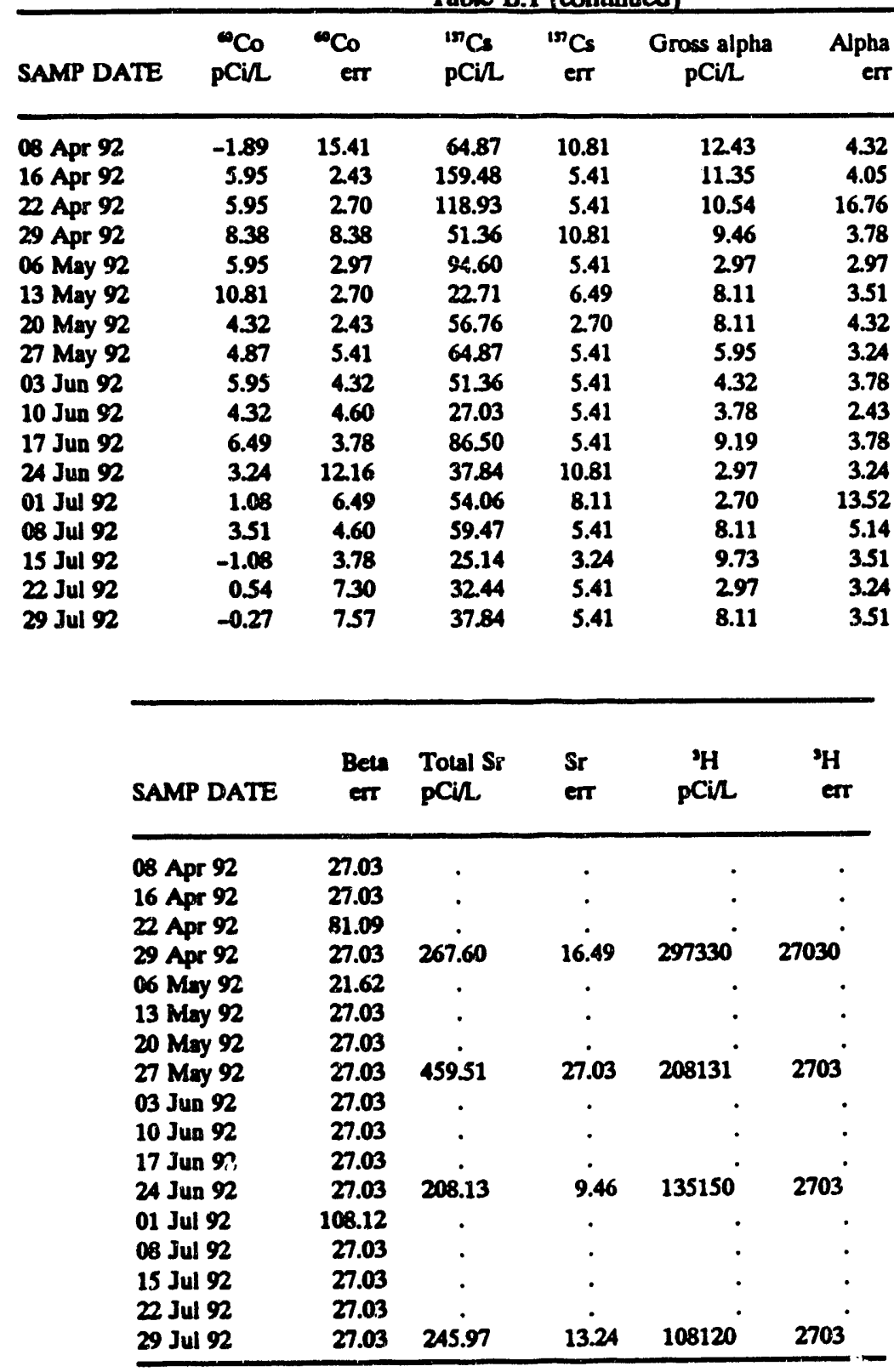


Table B.2. White Oak Dam physical-chemical parameters, June 1991 through July 1992 Data for temperature

(TEMP), conductivity (COND), turbidity (TURB), total dissolved solids (TDS), and total suspended solids (TSS) provided by ORNL Environmental Monitoring and Compliance.

\begin{tabular}{|c|c|c|c|c|}
\hline Month & Date & Analysis & Value & Units \\
\hline $\begin{array}{l}6 \\
6 \\
6 \\
6 \\
6 \\
6 \\
6 \\
6 \\
6\end{array}$ & $\begin{array}{l}\text { 04 Jun 91 } \\
\text { 06 Jun 91 } \\
\text { 06 Jun 91 } \\
\text { 06 Jun 91 } \\
\text { 06 Jun 91 } \\
\text { 06 Jun 91 } \\
11 \text { Jun 91 } \\
\text { 18 Jun 91 } \\
\text { 25 Jun 91 }\end{array}$ & $\begin{array}{l}\text { TEMP } \\
\text { COND } \\
\text { TDS } \\
\text { TEMP } \\
\text { TSS } \\
\text { TURB } \\
\text { TEMP } \\
\text { TEMP } \\
\text { TEMP }\end{array}$ & $\begin{array}{r}26.1 \\
1.6 \\
175.0 \\
25.5 \\
19.0 \\
30.0 \\
25.2 \\
29.3 \\
23.5\end{array}$ & $\begin{array}{l}{ }^{\circ} \mathrm{C} \\
\mathrm{mS} / \mathrm{cm} \\
\mathrm{mg} / \mathrm{L} \\
{ }^{\circ} \mathrm{C} \\
\mathrm{mg} / \mathrm{L} \\
\mathrm{JTU} \\
{ }^{\circ} \mathrm{C} \\
{ }^{\circ} \mathrm{C} \\
{ }^{\circ} \mathrm{C}\end{array}$ \\
\hline $\begin{array}{l}7 \\
7 \\
7 \\
7 \\
7 \\
7 \\
7 \\
7 \\
7 \\
7\end{array}$ & $\begin{array}{l}\text { 02 Jul } 91 \\
09 \text { Jul } 91 \\
11 \text { Jul } 91 \\
11 \text { Jul } 91 \\
11 \text { Jul } 91 \\
11 \text { Jul } 91 \\
11 \text { Jul } 91 \\
16 \text { Jul } 91 \\
23 \text { Jul } 91 \\
\text { 30 Jul } 91\end{array}$ & $\begin{array}{l}\text { TEMP } \\
\text { TEMP } \\
\text { COND } \\
\text { TDS } \\
\text { TEMP } \\
\text { TSS } \\
\text { TURB } \\
\text { TEMP } \\
\text { TEMP } \\
\text { TEMP }\end{array}$ & $\begin{array}{r}27.4 \\
27.2 \\
1.8 \\
231.0 \\
28.7 \\
29.0 \\
22.0 \\
27.7 \\
29.7 \\
27.1\end{array}$ & $\begin{array}{l}{ }^{\circ} \mathrm{C} \\
{ }^{\circ} \mathrm{C} \\
\mathrm{mS} / \mathrm{cm} \\
\mathrm{mg} / \mathrm{L} \\
{ }^{\circ} \mathrm{C} \\
\mathrm{mg} / \mathrm{L} \\
\mathrm{JTU} \\
{ }^{\circ} \mathrm{C} \\
{ }^{\circ} \mathrm{C} \\
{ }^{\circ} \mathrm{C}\end{array}$ \\
\hline $\begin{array}{l}8 \\
8 \\
8 \\
8 \\
8 \\
8 \\
8 \\
8 \\
8\end{array}$ & $\begin{array}{l}06 \text { Aug } 91 \\
08 \text { Aug } 91 \\
08 \text { Aug } 91 \\
08 \text { Aug } 91 \\
08 \text { Aug } 91 \\
08 \text { Aug } 91 \\
13 \text { Aug } 91 \\
20 \text { Aug } 91 \\
27 \text { Aug } 91\end{array}$ & $\begin{array}{l}\text { TEMP } \\
\text { COND } \\
\text { TDS } \\
\text { TEMP } \\
\text { TSS } \\
\text { TURB } \\
\text { TEMP } \\
\text { TEMP } \\
\text { TEMP }\end{array}$ & $\begin{array}{r}25.10 \\
0.36 \\
179.00 \\
28.20 \\
39.00 \\
16.00 \\
24.40 \\
24.60 \\
24.60\end{array}$ & $\begin{array}{l}{ }^{\circ} \mathrm{C} \\
\mathrm{mS} / \mathrm{cm} \\
\mathrm{mg} / \mathrm{L} \\
{ }^{\circ} \mathrm{C} \\
\mathrm{mg} / \mathrm{L} \\
\mathrm{JTU} \\
{ }^{\circ} \mathrm{C} \\
{ }^{\circ} \mathrm{C} \\
{ }^{\circ} \mathrm{C}\end{array}$ \\
\hline
\end{tabular}


Table B.2 (continued)

\begin{tabular}{|c|c|c|c|c|}
\hline Month & Date & Analysis & Value & Units \\
\hline $\begin{array}{l}9 \\
9 \\
9 \\
9 \\
9 \\
9 \\
9 \\
9 \\
9\end{array}$ & $\begin{array}{l}04 \text { Sep } 91 \\
05 \text { Sep } 91 \\
05 \text { Sep } 91 \\
05 \text { Sep } 91 \\
05 \text { Sep } 91 \\
05 \text { Sep } 91 \\
10 \text { Sep } 91 \\
17 \text { Sep } 91 \\
24 \text { Sep } 91\end{array}$ & $\begin{array}{l}\text { TEMP } \\
\text { COND } \\
\text { TDS } \\
\text { TEMP } \\
\text { TSS } \\
\text { TURB } \\
\text { TEMP } \\
\text { TEMP } \\
\text { TEMP }\end{array}$ & $\begin{array}{r}24.6 \\
1.6 \\
229.0 \\
25.2 \\
16.0 \\
10.0 \\
25.4 \\
26.5 \\
19.2\end{array}$ & $\begin{array}{l}{ }^{\circ} \mathrm{C} \\
\mathrm{mS} / \mathrm{cm} \\
\mathrm{mg} / \mathrm{L} \\
{ }^{\circ} \mathrm{C} \\
\mathrm{mg} / \mathrm{L} \\
\mathrm{JTU} \\
{ }^{\circ} \mathrm{C} \\
{ }^{\circ} \mathrm{C} \\
{ }^{\circ} \mathrm{C}\end{array}$ \\
\hline $\begin{array}{l}10 \\
10 \\
10 \\
10 \\
10 \\
10 \\
10 \\
10 \\
10 \\
10\end{array}$ & $\begin{array}{l}01 \text { Oct } 91 \\
03 \text { Oct } 91 \\
03 \text { Oct } 91 \\
03 \text { Oct } 91 \\
03 \text { Oct } 91 \\
03 \text { Oct } 91 \\
08 \text { Oct } 91 \\
15 \text { Oct } 91 \\
22 \text { Oct } 91 \\
29 \text { Oct } 91\end{array}$ & $\begin{array}{l}\text { TEMP } \\
\text { COND } \\
\text { TDS } \\
\text { TEMP } \\
\text { TSS } \\
\text { TURB } \\
\text { TEMP } \\
\text { TEMP } \\
\text { TEMP } \\
\text { TEMP }\end{array}$ & $\begin{array}{r}18.0 \\
1.4 \\
209.0 \\
19.4 \\
15.0 \\
18.0 \\
14.5 \\
15.0 \\
14.6 \\
20.4\end{array}$ & $\begin{array}{l}{ }^{\circ} \mathrm{C} \\
\mathrm{mS} / \mathrm{cm} \\
\mathrm{mg} / \mathrm{L} \\
{ }^{\circ} \mathrm{C} \\
\mathrm{mg} / \mathrm{L} \\
\mathrm{JTU} \\
{ }^{\circ} \mathrm{C} \\
{ }^{\circ} \mathrm{C} \\
{ }^{\circ} \mathrm{C} \\
{ }^{\circ} \mathrm{C}\end{array}$ \\
\hline $\begin{array}{l}11 \\
11 \\
11 \\
11 \\
11 \\
11 \\
11 \\
11 \\
11\end{array}$ & $\begin{array}{l}05 \text { Nov } 91 \\
07 \text { Nov } 91 \\
07 \text { Nov } 91 \\
07 \text { Nov } 91 \\
07 \text { Nov } 91 \\
07 \text { Nov } 91 \\
12 \text { Nov } 91 \\
19 \text { Nov } 91 \\
26 \text { Nov } 91\end{array}$ & $\begin{array}{l}\text { TEMP } \\
\text { COND } \\
\text { TDS } \\
\text { TEMP } \\
\text { TSS } \\
\text { TURB } \\
\text { TEMP } \\
\text { TEMP } \\
\text { TEMP }\end{array}$ & $\begin{array}{r}9.0 \\
0.4 \\
258.0 \\
9.6 \\
8.0 \\
6.0 \\
8.8 \\
13.8 \\
6.2\end{array}$ & $\begin{array}{l}{ }^{\circ} \mathrm{C} \\
\mathrm{mS} / \mathrm{cm} \\
\mathrm{mg} / \mathrm{L} \\
{ }^{\circ} \mathrm{C} \\
\mathrm{mg} / \mathrm{L} \\
\mathrm{JTU} \\
{ }^{\circ} \mathrm{C} \\
{ }^{\circ} \mathrm{C} \\
{ }^{\circ} \mathrm{C}\end{array}$ \\
\hline
\end{tabular}




\section{B-8}

Table B.2 (continued)

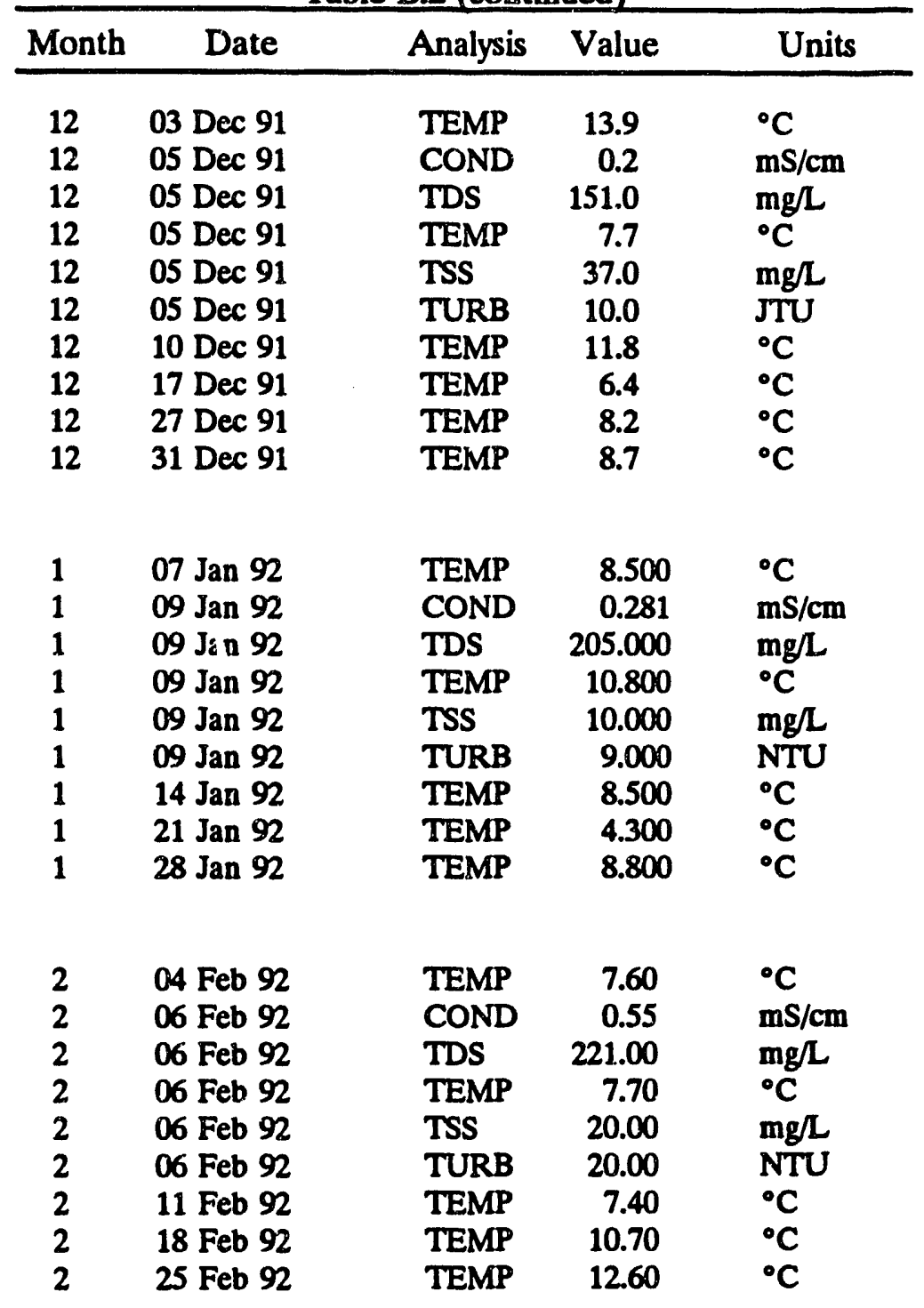




\section{B-9}

Table B.2 (continued)

\begin{tabular}{|c|c|c|c|c|}
\hline Month & Date & Analysis & Value & Units \\
\hline 3 & 03 Mar 92 & TEMP & 12.4 & ${ }^{\circ} \mathrm{C}$ \\
\hline 3 & $05 \operatorname{Mar} 92$ & COND & 0.7 & $\mathrm{mS} / \mathrm{cm}$ \\
\hline 3 & 05 Mar 92 & TDS & 194.0 & $\mathrm{mg} / \mathrm{L}$ \\
\hline 3 & 05 Mar 92 & TEMP & 14.9 & ${ }^{\circ} \mathbf{C}$ \\
\hline 3 & 05 Mar 92 & TSS & 15.0 & $\mathbf{m g} / \mathbf{L}$ \\
\hline 3 & 05 Mar 92 & TURB & 27.0 & NTU \\
\hline 3 & $10 \operatorname{Mar} 92$ & TEMP & 16.5 & ${ }^{\circ} \mathrm{C}$ \\
\hline 3 & 17 Mar 92 & TEMP & 11.1 & ${ }^{\circ} \mathrm{C}$ \\
\hline 3 & $24 \operatorname{Mar} 92$ & TEMP & 10.4 & ${ }^{\circ} \mathrm{C}$ \\
\hline 3 & 31 Mar 92 & TEMP & 11.1 & ${ }^{\circ} \mathrm{C}$ \\
\hline 4 & 07 Apr 92 & TEMP & 12.9 & ${ }^{\circ} \mathrm{C}$ \\
\hline 4 & 09 Apr 92 & COND & 0.2 & $\mathrm{mS} / \mathrm{cm}$ \\
\hline 4 & 09 Apr 92 & TDS & 279.0 & $\mathrm{mg} / \mathrm{L}$ \\
\hline 4 & 09 Apr 92 & TEMP & 15.5 & ${ }^{\circ} \mathbf{C}$ \\
\hline 4 & 09 Apr 92 & TSS & 13.0 & $\mathrm{mg} / \mathrm{L}$ \\
\hline 4 & 09 Apr 92 & TURB & 18.0 & NTU \\
\hline 4 & 14 Apr 92 & TEMP & 17.7 & ${ }^{\circ} \mathrm{C}$ \\
\hline 4 & 21 Apr 92 & TEMP & 21.2 & ${ }^{\circ} \mathrm{C}$ \\
\hline 4 & 28 Apr 92 & TEMP & 13.7 & ${ }^{\circ} \mathrm{C}$ \\
\hline 5 & 05 May 92 & TEMP & 19.2 & ${ }^{\circ} \mathrm{C}$ \\
\hline 5 & 07 May 92 & COND & 0.9 & $\mathrm{mS} / \mathrm{cm}$ \\
\hline 5 & 07 May 92 & TDS & 255.0 & $\mathrm{mg} / \mathrm{L}$ \\
\hline 5 & 07 May 92 & TEMP & 15.5 & ${ }^{\circ} \mathrm{C}$ \\
\hline 5 & 07 May 92 & TSS & 39.0 & $\mathrm{mg} / \mathrm{L}$ \\
\hline 5 & 07 May 92 & TURB & 18.0 & NTU \\
\hline 5 & 12 May 92 & TEMP & 22.8 & ${ }^{\circ} \mathrm{C}$ \\
\hline 5 & 19 May 92 & TEMP & 26.2 & ${ }^{\circ} \mathrm{C}$ \\
\hline 5 & 26 May 92 & TEMP & 21.4 & ${ }^{\circ} \mathrm{C}$ \\
\hline
\end{tabular}


B-10

Table B.2 (continued)

\begin{tabular}{lllrl}
\hline Month & Date & Analysis & Value & Units \\
\hline 6 & 02 Jun 92 & TEMP & 20.00 & ${ }^{\circ} \mathrm{C}$ \\
6 & 04 Jun 92 & COND & 0.16 & $\mathrm{mS} / \mathrm{cm}$ \\
6 & 04 Jun 92 & TDS & 279.00 & $\mathrm{mg} / \mathrm{L}$ \\
6 & 04 Jun 92 & TEMP & 20.80 & ${ }^{\circ} \mathrm{C}$ \\
6 & 04 Jun 92 & TSS & 14.00 & $\mathrm{mg} / \mathrm{L}$ \\
6 & 04 Jun 92 & TURB & 18.00 & $\mathrm{NTU}$ \\
6 & 09 Jun 92 & TEMP & 24.20 & ${ }^{\circ} \mathrm{C}$ \\
6 & 16 Jun 92 & TEMP & 27.90 & ${ }^{\circ} \mathrm{C}$ \\
6 & 23 Jun 92 & TEMP & 22.80 & ${ }^{\circ} \mathrm{C}$ \\
6 & 29 Jun 92 & TEMP & 24.50 & ${ }^{\circ} \mathrm{C}$ \\
& & & & \\
7 & 07 Jul 92 & TEMP & 25.20 & ${ }^{\circ} \mathrm{C}$ \\
7 & 09 Jul 92 & COND & 0.12 & $\mathrm{mS} / \mathrm{cm}$ \\
7 & 09 Jul 92 & TDS & 220.00 & $\mathrm{mg} / \mathrm{L}$ \\
7 & 09 Jul 92 & TEMP & 27.50 & ${ }^{\circ} \mathrm{C}$ \\
7 & 09 Jul 92 & TSS & 7.00 & $\mathrm{mg} / \mathrm{L}$ \\
7 & 09 Jul 92 & TURB & 8.00 & $\mathrm{NTU}$ \\
7 & 14 Jul 92 & TEMP & 27.40 & ${ }^{\circ} \mathrm{C}$ \\
7 & 21 Jul 92 & TEMP & 27.10 & ${ }^{\circ} \mathrm{C}$ \\
7 & 28 Jul 92 & TEMP & 25.50 & ${ }^{\circ} \mathrm{C}$ \\
\hline
\end{tabular}


Appendix C

CONTINUOUS WATER QUALITY MONITORING RESULTS 


\section{C-3}

Table C.1. Water quality monitoring results for the White Oak Creek mouth station (CRM 20.8).

Data are hourly average values.

\begin{tabular}{|c|c|c|c|c|}
\hline Date & Hour & $\begin{array}{c}\text { Temperature } \\
\left({ }^{\circ} \mathrm{C}\right)\end{array}$ & $\mathrm{pH}$ & $\begin{array}{c}\text { Dissolved } \\
\text { axygen } \\
\text { (mg/L) }\end{array}$ \\
\hline 04 Jun 91 & 0 & 20.9143 & 6.9929 & 4.7329 \\
\hline 04 Jun 91 & 1 & 21.1243 & 7.1514 & 4.0329 \\
\hline 04 Jun 91 & 2 & 21.0171 & 7.2357 & 3.8914 \\
\hline O4 Jun 91 & 3 & 20.9050 & 7.0717 & 4.1200 \\
\hline 04 Jun 91 & 4 & 20.8250 & 7.0600 & 4.2450 \\
\hline 04 Jun 91 & 5 & 20.6333 & 7.0517 & 4.2533 \\
\hline 04 Jun 91 & 6 & 20.5533 & 7.0567 & 4.2267 \\
\hline 04 Jun 91 & 7 & 20.4667 & 7.0467 & 4.3917 \\
\hline 04 Jun 91 & 8 & 20.3083 & 7.0400 & 4.4867 \\
\hline O4 Jun 91 & 9 & 20.3067 & 7.0717 & 4.5867 \\
\hline 04 Jun 91 & 10 & 20.3950 & 7.0450 & 4.2617 \\
\hline 04 Jun 91 & 11 & 20.6017 & 7.0283 & 4.1183 \\
\hline 04 Jun 91 & 12 & 20.8467 & 7.0300 & 4.1917 \\
\hline 04 Jun 91 & 13 & 20.9333 & 7.0333 & 4.1333 \\
\hline O4 Jun 91 & 14 & 20.2850 & 6.8375 & 4.9775 \\
\hline 04 Jun 91 & 15 & 20.0117 & 6.6933 & 5.9033 \\
\hline 04 Jun 91 & 16 & 19.2260 & 6.2680 & 7.4880 \\
\hline 04 Jun 91 & 17 & 20.1240 & 6.5980 & 7.1120 \\
\hline O4 Jun 91 & 18 & 20.5883 & 6.7700 & 6.6100 \\
\hline 04 Jun 91 & 19 & 21.2783 & 6.8500 & 6.5050 \\
\hline 04 Jun 91 & 20 & 21.1350 & 6.8200 & 6.2933 \\
\hline 04 Jun 91 & 21 & 21.1529 & 5.9871 & 5.8857 \\
\hline 04 Jun 91 & 22 & 21.0038 & 6.9100 & 5.1175 \\
\hline 04 Jun 91 & 23 & 20.8463 & 6.8900 & 5.0363 \\
\hline 13 Jun 91 & $\mathbf{0}$ & 19.5800 & 6.2400 & 7.3300 \\
\hline 13 Jun 91 & 1 & 19.5400 & 6.2400 & 7.3900 \\
\hline 13 Jun 91 & 2 & 19.5600 & 6.2250 & 7.2800 \\
\hline 13 Jun 91 & 3 & 19.5650 & 6.2000 & 7.0850 \\
\hline 13 Jun 91 & 4 & 19.5700 & 6.2300 & 7.3900 \\
\hline 13 Jun 91 & 5 & 19.7400 & 6.3150 & 7.5400 \\
\hline
\end{tabular}




\section{C-4}

Table C.1 (continued)

\begin{tabular}{|c|c|c|c|c|}
\hline Date & Hour & $\begin{array}{c}\text { Temperature } \\
\left({ }^{\circ} \mathrm{C}\right)\end{array}$ & $\mathrm{pH}$ & $\begin{array}{c}\text { Dissolved } \\
\text { arygen } \\
(\mathrm{mg} / \mathrm{L})\end{array}$ \\
\hline 13 Jun 91 & 6 & 19.7850 & 6.3000 & 7.4350 \\
\hline 13 Jun 91 & 7 & 19.9100 & 6.2500 & 7.3000 \\
\hline 13 Jun 91 & 8 & 19.3950 & 6.3200 & 7.6400 \\
\hline 13 Jun 91 & 9 & 19.4000 & 6.4300 & 7.5050 \\
\hline 13 Jun 91 & 10 & 19.4550 & 6.4700 & 7.4900 \\
\hline 13 Jun 91 & 11 & 19.7850 & 6.4450 & 7.2450 \\
\hline 13 Jun 91 & 12 & 20.2000 & 6.4200 & 7.2900 \\
\hline 13 Jun 91 & 13 & 19.9867 & 6.4200 & 7.3367 \\
\hline 13 Jun 91 & 14 & 20.1550 & 6.5300 & 7.5700 \\
\hline 13 Jun 91 & 15 & 20.2000 & 6.3300 & 7.4600 \\
\hline 13 Jun 91 & 16 & 20.2800 & 6.2800 & 7.4200 \\
\hline 13 Jun 91 & 17 & 20.5300 & 6.2500 & 7.2250 \\
\hline 13 Jun 91 & 18 & 20.4700 & 6.3700 & 7.2800 \\
\hline 13 Jun 91 & 19 & 21.3000 & 6.1550 & 6.7750 \\
\hline 13 Jun 91 & 20 & 20.0750 & 6.3650 & 7.2950 \\
\hline 13 Jun 91 & 21 & 20.0000 & 6.3150 & 7.2350 \\
\hline 13 Jun 91 & 22 & 19.9950 & 6.2900 & 7.1000 \\
\hline 13 Jun 91 & 23 & 19.9950 & 6.3150 & 7.2350 \\
\hline 14 Jun 91 & 0 & 20.0000 & 6.2700 & 6.9250 \\
\hline 14 Jun 91 & 1 & 19.9850 & 6.2250 & 7.0500 \\
\hline 14 Jun 91 & 2 & 19.9900 & 6.2150 & 6.8950 \\
\hline 14 Jun 91 & 3 & 20.0000 & 6.2000 & 6.6900 \\
\hline 14 Jun 91 & 4 & 19.9850 & 6.2000 & 6.8050 \\
\hline 14 Jun 91 & 5 & 19.9800 & 6.2100 & 6.9600 \\
\hline 14 Jun 91 & 6 & 19.9850 & 6.1850 & 6.7300 \\
\hline 14 Jun 91 & 7 & 19.9800 & 6.2550 & 6.8450 \\
\hline 14 Jun 91 & 8 & 19.9850 & 6.1750 & 6.7400 \\
\hline 14 Jun 91 & 9 & 19.9900 & 6.1650 & 6.7400 \\
\hline 14 Jun 91 & 10 & 19.9900 & 6.1650 & 6.5700 \\
\hline 14 Jun 91 & 11 & 20.0050 & 6.1750 & 6.3600 \\
\hline 14 Jun 91 & 12 & 19.9900 & 6.2050 & 6.5300 \\
\hline 14 Jun 91 & 13 & 20.3100 & 6.3150 & 7.1100 \\
\hline
\end{tabular}




\section{C-5}

Table C.1 (continued)

\begin{tabular}{|c|c|c|c|c|}
\hline Date & Hour & $\begin{array}{c}\text { Temperature } \\
\left({ }^{\circ} \mathrm{C}\right)\end{array}$ & pH & $\begin{array}{c}\text { Dissolved } \\
\text { oxygen } \\
(\mathrm{mg} / \mathrm{L})\end{array}$ \\
\hline 14 Jun 91 & 14 & 20.6650 & 6.3850 & 7.4000 \\
\hline 14 Jun 91 & 15 & 20.3150 & 6.2650 & 7.4600 \\
\hline 14 Jun 91 & 16 & 20.1800 & 6.3450 & 7.3550 \\
\hline 14 Jun 91 & 17 & 20.1700 & 6.2150 & 7.2100 \\
\hline 14 Jun 91 & 18 & 20.1050 & 6.2450 & 7.1400 \\
\hline 14 Jun 91 & 19 & 20.1300 & 6.3200 & 7.1900 \\
\hline 14 Jun 91 & 20 & 19.8500 & 6.2950 & 7.0750 \\
\hline 14 Jun 91 & 21 & 19.7700 & 6.2400 & 7.0250 \\
\hline 14 Jun 91 & 22 & 19.7300 & 6.2650 & 6.8450 \\
\hline 14 Jun 91 & 23 & 19.7300 & 6.1700 & 6.8700 \\
\hline 15 Jun 91 & 0 & 19.7125 & 6.2000 & 6.4200 \\
\hline 15 Jun 91 & 1 & 19.7050 & 6.1000 & 6.3475 \\
\hline 15 Jun 91 & 2 & 19.6900 & 6.1650 & 6.3950 \\
\hline 15 Jun 91 & 3 & 19.7067 & 6.2033 & 6.2800 \\
\hline 15 Jun 91 & 4 & 19.7025 & 6.1275 & 6.2475 \\
\hline 15 Jun 91 & 5 & 19.6950 & 6.1800 & 6.2000 \\
\hline 15 Jun 91 & 7 & 19.7000 & 6.1080 & 6.0620 \\
\hline 15 Jun 91 & 8 & 19.6900 & 6.1475 & 6.1800 \\
\hline 15 Jun 91 & 9 & 19.6925 & 6.1450 & 6.1900 \\
\hline 15 Jun 91 & 10 & 19.7025 & 6.1525 & 6.3900 \\
\hline 15 Jun 91 & 11 & 19.7175 & 6.1650 & 6.6100 \\
\hline 15 Jun 91 & 12 & 19.7350 & 6.1900 & 6.4475 \\
\hline 15 Jun 91 & 13 & 19.7500 & 6.1650 & 6.2900 \\
\hline 15 Jun 91 & 14 & 20.1175 & 6.2475 & 7.0325 \\
\hline 15 Jun 91 & 15 & 20.2875 & 6.2600 & 6.9200 \\
\hline 15 Jun 91 & 16 & 20.2150 & 6.3100 & 6.9825 \\
\hline 15 Jun 91 & 17 & 20.0760 & 6.2820 & 7.0640 \\
\hline 15 Jun 91 & 18 & 20.7550 & 6.3075 & 6.9500 \\
\hline 15 Jun 91 & 19 & 20.8025 & 6.2825 & 6.7625 \\
\hline 15 Jun 91 & 20 & 19.8400 & 6.2150 & 6.6500 \\
\hline 15 Jun 91 & 21 & 19.7825 & 6.2150 & 6.5325 \\
\hline 15 Jun 91 & 22 & 19.7900 & 6.1700 & 6.3100 \\
\hline
\end{tabular}




\section{C-6}

Table C.1 (continued)

\begin{tabular}{|c|c|c|c|c|}
\hline Date & Hour & $\begin{array}{c}\text { Temperature } \\
\left({ }^{\circ} \mathrm{C}\right)\end{array}$ & $\mathrm{pH}$ & $\begin{array}{c}\text { Dissolved } \\
\text { oxygen } \\
(\mathrm{mg} / \mathrm{L})\end{array}$ \\
\hline 15 Jun 91 & 23 & 19.8100 & 6.1500 & 6.3175 \\
\hline 16 Jun 91 & 0 & & & \\
\hline 17 Jun 91 & 0 & 19.8700 & 6.1200 & 5.9450 \\
\hline 17 Jun 91 & 1 & 19.8550 & 6.1250 & 5.9450 \\
\hline 17 Jun 91 & 2 & 19.8550 & 6.1100 & 5.7900 \\
\hline 17 Jun 91 & 3 & 19.8550 & 6.1250 & 5.5350 \\
\hline 17 Jun 91 & 4 & 19.8050 & 6.2000 & 6.3000 \\
\hline 17 Jun 91 & 5 & 19.8250 & 6.1900 & 6.1650 \\
\hline 17 Jun 91 & 6 & 19.8450 & 6.2150 & 6.4000 \\
\hline 17 Jun 91 & 7 & 19.8600 & 6.1950 & 6.2300 \\
\hline 17 Jun 91 & 8 & 19.8100 & 6.1600 & 5.9650 \\
\hline 17 Jun 91 & 9 & 19.8150 & 6.1400 & 6.2050 \\
\hline 17 Jun 91 & 10 & 19.8450 & 6.1200 & 5.9250 \\
\hline 17 Jun 91 & 11 & 19.8600 & 6.0650 & 5.8900 \\
\hline 17 Jun 91 & 12 & 19.8650 & 6.0650 & 5.8400 \\
\hline 17 Jun 91 & 13 & 20.1050 & 6.2200 & 6.3750 \\
\hline 17 Jun 91 & 14 & 20.2900 & 6.2300 & 6.7200 \\
\hline 17 Jun 91 & 15 & 20.2900 & 6.1700 & 6.7450 \\
\hline 17 Jun 91 & 16 & 20.5150 & 6.2050 & 6.6550 \\
\hline 17 Jun 91 & 17 & 20.6200 & 6.1950 & 6.5200 \\
\hline 17 Jun 91 & 18 & 20.4950 & 6.1800 & 6.3350 \\
\hline 17 Jun 91 & 19 & 22.1550 & 6.4050 & 7.4050 \\
\hline 17 Jun 91 & 20 & 20.3000 & 6.1200 & 6.2100 \\
\hline 17 Jun 91 & 21 & 20.2800 & 6.0900 & 6.0650 \\
\hline 17 Jun 91 & 22 & 20.2700 & 5.9900 & 5.8800 \\
\hline 17 Jun 91 & 23 & 20.2450 & 6.0650 & 5.8500 \\
\hline 18 Jun 91 & $\mathbf{0}$ & 20.2450 & 6.0550 & 5.4950 \\
\hline 18 Jun 91 & 1 & 20.2400 & 6.0350 & 5.3450 \\
\hline 18 Jun 91 & 2 & 20.2500 & 5.9950 & 5.2400 \\
\hline 18 Jun 91 & 3 & 20.2400 & 6.0700 & 5.7400 \\
\hline 18 Jun 91 & 4 & 20.2500 & 6.0000 & 5.3550 \\
\hline 18 Jun 91 & 5 & 20.2400 & 6.0550 & 5.6500 \\
\hline
\end{tabular}


C-7

Table C.1 (continued)

\begin{tabular}{|c|c|c|c|c|}
\hline Date & Hour & $\begin{array}{l}\text { Temperature } \\
\left({ }^{\circ} \mathrm{C}\right)\end{array}$ & $\mathrm{pH}$ & $\begin{array}{l}\text { Dissolved } \\
\text { axygen } \\
(\mathrm{mg} / \mathrm{L})\end{array}$ \\
\hline 18 Jun 91 & 6 & 20.2400 & 6.0500 & 5.5100 \\
\hline 18 Jun 91 & 7 & 20.2250 & 6.0650 & 5.7300 \\
\hline 18 Jun 91 & 8 & 20.2500 & 6.0700 & 5.8000 \\
\hline 18 Jun 91 & 9 & 20.2550 & 6.0350 & 5.4950 \\
\hline 18 Jun 91 & 10 & 20.2500 & 6.0150 & 5.3900 \\
\hline 18 Jun 91 & 11 & 20.2750 & 6.0400 & 5.3950 \\
\hline 18 Jun 91 & 12 & 20.3000 & 6.0550 & 5.9050 \\
\hline 18 Jun 91 & 13 & 20.6000 & 6.2250 & 6.4250 \\
\hline 18 Jun 91 & 14 & 20.9000 & 6.2200 & 6.5100 \\
\hline 18 Jun 91 & 15 & 20.7600 & 6.2200 & 6.6200 \\
\hline 18 Jun 91 & 16 & 20.7300 & 6.1950 & 6.7400 \\
\hline 18 Jun 91 & 17 & 20.6450 & 6.2400 & 6.7350 \\
\hline 18 Jun 91 & 18 & 20.5300 & 6.2000 & 6.7600 \\
\hline 18 Jun 91 & 19 & 20.5950 & 6.2400 & 6.9400 \\
\hline 18 Jun 91 & 20 & 20.6250 & 6.3100 & 6.8400 \\
\hline 18 Jun 91 & 21 & 20.5200 & 6.2400 & 6.6500 \\
\hline 18 Jun 91 & 22 & 20.2600 & 6.1800 & 6.4150 \\
\hline 18 Jun 91 & 23 & 20.2350 & 6.1650 & 6.2950 \\
\hline 19 Jun 91 & 0 & 20.3275 & 6.2350 & 6.5175 \\
\hline 19 Jun 91 & 1 & 20.3600 & 6.2667 & 6.5967 \\
\hline 19 Jun 91 & 2 & 20.3175 & 6.2400 & 6.5350 \\
\hline 19 Jun 91 & 3 & 20.3125 & 6.2125 & 6.5175 \\
\hline 19 Jun 91 & 4 & 20.3075 & 6.2325 & 6.5250 \\
\hline 19 Jun 91 & 5 & 20.3200 & 6.2340 & 6.5140 \\
\hline 19 Jun 91 & 7 & 20.2900 & 6.2100 & 6.3675 \\
\hline 19 Jun 91 & 8 & 20.2925 & 6.2500 & 6.3175 \\
\hline 19 Jun 91 & 9 & 20.2975 & 6.1800 & 6.2175 \\
\hline 19 Jun 91 & 10 & 20.2950 & 6.1675 & 6.3075 \\
\hline 19 Jun 91 & 11 & 20.3050 & 6.1400 & 6.3050 \\
\hline 19 Jun 91 & 12 & 20.4375 & 6.1675 & 6.5025 \\
\hline 19 Jun 91 & 13 & 20.4850 & 6.1850 & 6.5050 \\
\hline 19 Jun 91 & 14 & 20.6100 & 6.2375 & 6.9625 \\
\hline
\end{tabular}




\section{C-8}

Table C.1 (continued)

\begin{tabular}{|c|c|c|c|c|}
\hline Date & Hour & $\begin{array}{c}\text { Temperature } \\
\left({ }^{\circ} \mathrm{C}\right)\end{array}$ & $\mathrm{pH}$ & $\begin{array}{l}\text { Dissolved } \\
\text { oxygen } \\
(\mathrm{mg} / \mathrm{L})\end{array}$ \\
\hline 19 Jun 91 & 15 & 20.7040 & 6.2800 & 6.9400 \\
\hline 19 Jun 91 & 16 & 20.7025 & 6.3025 & 6.9850 \\
\hline 19 Jun 91 & 17 & 21.0100 & 6.3850 & 7.1050 \\
\hline 19 Jun 91 & 18 & 21.0375 & 6.3525 & 7.1200 \\
\hline 19 Jun 91 & 19 & 20.9925 & 6.3725 & 7.2025 \\
\hline 19 Jun 91 & 20 & 21.6125 & 6.5350 & 7.7875 \\
\hline 19 Jun 91 & 21 & 21.5050 & 6.5025 & 7.7900 \\
\hline 19 Jun 91 & 22 & 20.6400 & 6.2550 & 7.0075 \\
\hline 19 Jun 91 & 23 & 20.6350 & 6.2550 & 6.9050 \\
\hline 20 Jun 91 & 0 & & & \\
\hline 21 Jun 91 & 0 & 21.0100 & 6.5150 & 7.5275 \\
\hline 21 Jun 91 & 1 & 21.0400 & 6.5360 & 7.5620 \\
\hline 21 Jun 91 & 2 & 20.9800 & 6.5175 & 7.4600 \\
\hline 21 Jun 91 & 3 & 20.9775 & 6.5000 & 7.3825 \\
\hline 21 Jun 91 & 4 & 20.9800 & 6.4725 & 7.4450 \\
\hline 21 Jun 91 & 5 & 20.9775 & 6.5000 & 7.4200 \\
\hline 21 Jun 91 & 6 & 20.9675 & 6.4675 & 7.3200 \\
\hline 21 Jun 91 & 7 & 20.9600 & 6.4525 & 7.2200 \\
\hline 21 Jun 91 & 8 & 20.9625 & 6.4675 & 7.2300 \\
\hline 21 Jun 91 & 9 & 20.9625 & 6.4775 & 7.3400 \\
\hline 21 Jun 91 & 10 & 20.9550 & 6.4600 & 7.2700 \\
\hline 21 Jun 91 & 11 & 20.9200 & 6.4800 & 7.1850 \\
\hline 21 Jun 91 & 12 & 20.9150 & 6.4925 & 7.2200 \\
\hline 21 Jun 91 & 13 & 20.9200 & 6.5275 & 7.2250 \\
\hline 21 Jun 91 & 14 & 21.2025 & 6.5600 & 7.6375 \\
\hline 21 Jun 91 & 15 & 21.1650 & 6.5950 & 7.7125 \\
\hline 21 Jun 91 & 16 & 20.7120 & 6.6280 & 7.9400 \\
\hline 21 Jun 91 & 17 & 20.4875 & 6.6250 & 7.6325 \\
\hline 21 Jun 91 & 18 & 20.4075 & 6.6250 & 7.4275 \\
\hline 21 Jun 91 & 19 & 21.5075 & 6.8150 & 8.6075 \\
\hline 21 Jun 91 & 20 & 20.8800 & 6.6950 & 7.7050 \\
\hline 21 Jun 91 & 21 & 20.7600 & 6.6800 & 7.5800 \\
\hline
\end{tabular}


C-9

Table C.1 (continued)

\begin{tabular}{|c|c|c|c|c|}
\hline Date & Hour & $\begin{array}{c}\text { Temperature } \\
\left({ }^{\circ} \mathrm{C}\right)\end{array}$ & pH & $\begin{array}{c}\text { Dissolved } \\
\text { arygen } \\
(m g / L)\end{array}$ \\
\hline 21 Jun 91 & 22 & 20.7450 & 6.6925 & 7.5450 \\
\hline 21 Jun 91 & 23 & 20.7425 & 6.7025 & 7.5225 \\
\hline 22 Jun 91 & 0 & & & \\
\hline 23 Jun 91 & 0 & 20.3625 & 6.6300 & 6.8850 \\
\hline 23 Jun 91 & 1 & 20.3425 & 6.6350 & 6.8825 \\
\hline 23 Jun 91 & 2 & 20.3275 & 6.6400 & 6.8625 \\
\hline 23 Jun 91 & 3 & 20.3325 & 6.6300 & 6.8200 \\
\hline 23 Jun 91 & 4 & 20.3300 & 6.6375 & 6.8000 \\
\hline 23 Jun 91 & 5 & 20.3500 & 6.6300 & 6.7600 \\
\hline 23 Jun 91 & 6 & 20.3175 & 6.6225 & 6.7325 \\
\hline 23 Jun 91 & 7 & 20.3200 & 6.6275 & 6.7425 \\
\hline 23 Jun 91 & 8 & 20.3225 & 6.6350 & 6.7625 \\
\hline 23 Jun 91 & 9 & 20.3400 & 6.6250 & 6.7425 \\
\hline 23 Jun 91 & 10 & 20.3400 & 6.6080 & 6.7100 \\
\hline 23 Jun 91 & 11 & 20.4150 & 6.6425 & 6.8575 \\
\hline 23 Jun 91 & 12 & 20.3400 & 6.6375 & 6.7875 \\
\hline 23 Jun 91 & 13 & 20.7800 & 6.7000 & 7.5750 \\
\hline 23 Jun 91 & 14 & 21.0250 & 6.7450 & 7.6250 \\
\hline 23 Jun 91 & 15 & 20.5000 & 6.6450 & 7.2050 \\
\hline 23 Jun 91 & 16 & 20.7350 & 6.6800 & 7.3150 \\
\hline 23 Jun 91 & 17 & 20.6750 & 6.6650 & 7.0850 \\
\hline 23 Jun 91 & 18 & 20.5850 & 6.6600 & 7.0450 \\
\hline 23 Jun 91 & 19 & 20.7250 & 6.7050 & 7.2500 \\
\hline 23 Jun 91 & 20 & 21.4567 & 6.8467 & 7.7600 \\
\hline 23 Jun 91 & 21 & 20.4550 & 6.6450 & 6.9700 \\
\hline 23 Jun 91 & 22 & 20.4450 & 6.6300 & 6.9300 \\
\hline 23 Jun 91 & 23 & 20.3800 & 6.6600 & 6.8800 \\
\hline 24 Jun 91 & 13 & 20.8100 & 6.6900 & 7.3500 \\
\hline 24 Jun 91 & 14 & 20.7550 & 6.6700 & 7.1250 \\
\hline 24 Jun 91 & 15 & 20.5750 & 6.6600 & 7.1250 \\
\hline 24 Jun 91 & 16 & 20.5800 & 6.6950 & 7.1450 \\
\hline 24 Jun 91 & 17 & 20.8700 & 6.7500 & 7.1600 \\
\hline
\end{tabular}




\section{C-10}

Table C.1 (continued)

\begin{tabular}{|c|c|c|c|c|}
\hline Date & Hour & $\begin{array}{c}\text { Temperature } \\
\left({ }^{\circ} \mathrm{C}\right)\end{array}$ & pH & $\begin{array}{l}\text { Dissolved } \\
\text { oxygen } \\
(\mathrm{mg} / \mathrm{L})\end{array}$ \\
\hline 24 Jun 91 & 18 & 20.7550 & 6.7200 & 7.0550 \\
\hline 24 Jun 91 & 19 & 20.7500 & 6.7300 & 7.0550 \\
\hline 24 Jun 91 & 20 & 20.8200 & 6.7350 & 7.1150 \\
\hline 24 Jun 91 & 21 & 21.5500 & 6.7700 & 7.5600 \\
\hline 24 Jun 91 & 22 & 20.7750 & 6.7250 & 7.1100 \\
\hline 24 Jun 91 & 23 & 20.7600 & 6.7150 & 7.0700 \\
\hline 25 Jun 91 & 0 & 20.6700 & 6.7000 & 6.9750 \\
\hline 25 Jun 91 & 1 & 20.6550 & 6.7000 & 6.9650 \\
\hline 25 Jun 91 & 2 & 20.6850 & 6.6900 & 6.8750 \\
\hline 25 Jun 91 & 3 & 20.6500 & 6.6650 & 6.8850 \\
\hline 25 Jun 91 & 4 & 20.6750 & 6.6700 & 6.9000 \\
\hline 25 Jun 91 & 5 & 20.6850 & 6.6900 & 6.8950 \\
\hline 25 Jun 91 & 6 & 20.6800 & 6.6950 & 6.8500 \\
\hline 25 Jun 91 & 7 & 20.6500 & 6.6700 & 6.8350 \\
\hline 25 Jun 91 & 8 & 20.9100 & 6.6650 & 6.9350 \\
\hline 25 Jun 91 & 9 & 21.0467 & 6.6100 & 6.8200 \\
\hline 25 Jun 91 & 10 & 21.0750 & 6.5550 & 6.5900 \\
\hline 25 Jun 91 & 11 & 21.2600 & 6.5000 & 6.6300 \\
\hline 25 Jun 91 & 12 & 21.6350 & 6.4500 & 6.5850 \\
\hline 25 Jun 91 & 13 & 20.5450 & 6.5950 & 6.5700 \\
\hline 25 Jun 91 & 14 & 20.6950 & 6.6700 & 6.9300 \\
\hline 25 Jun 91 & 15 & 21.1450 & 6.7200 & 7.0600 \\
\hline 25 Jun 91 & 16 & 21.1150 & 6.6300 & 6.7250 \\
\hline 25 Jun 91 & 17 & 21.4800 & 6.5550 & 6.7500 \\
\hline 25 Jun 91 & 18 & 21.7650 & 6.5600 & 6.6150 \\
\hline 25 Jun 91 & 19 & 21.7350 & 6.6550 & 6.9750 \\
\hline 25 Jun 91 & 20 & 21.8400 & 6.5900 & 6.7950 \\
\hline 25 Jun 91 & 21 & 21.8250 & 6.5400 & 6.8250 \\
\hline 25 Jun 91 & 22 & 21.7150 & 6.3200 & 6.5000 \\
\hline 25 Jun 91 & 23 & 21.5200 & 6.3550 & 6.7300 \\
\hline 26 Jun 91 & 0 & 21.1575 & 6.4025 & 7.0300 \\
\hline 26 Jun 91 & 1 & 20.6050 & 6.2600 & 7.0275 \\
\hline
\end{tabular}




\section{C-11}

Table C.1 (continued)

\begin{tabular}{|c|c|c|c|c|}
\hline Date & Hour & $\begin{array}{l}\text { Temperature } \\
\left({ }^{\circ} \mathrm{C}\right)\end{array}$ & $\mathrm{pH}$ & $\begin{array}{l}\text { Dissolved } \\
\text { oxygen } \\
\text { (mg/L) }\end{array}$ \\
\hline 26 Jun 91 & 2 & 20.7600 & 6.3050 & 7.0950 \\
\hline 26 Jun 91 & 3 & 20.5650 & 6.2550 & 7.0450 \\
\hline 26 Jun 91 & 4 & 20.3750 & 6.2450 & 7.2200 \\
\hline 26 Jun 91 & 5 & 20.2300 & 6.2850 & 7.4950 \\
\hline 26 Jun 91 & 6 & 20.1700 & 6.2900 & 7.5050 \\
\hline 26 Jun 91 & 7 & 20.1500 & 6.2800 & 7.4100 \\
\hline 26 Jun 91 & 8 & 20.1200 & 6.2650 & 7.3200 \\
\hline 26 Jun 91 & 9 & 22.0950 & 6.7450 & 7.1100 \\
\hline 26 Jun 91 & 10 & 21.7250 & 6.6500 & 7.0750 \\
\hline 26 Jun 91 & 11 & 20.4750 & 6.3150 & 7.1950 \\
\hline 26 Jun 91 & 12 & 20.1850 & 6.2550 & 7.2200 \\
\hline 26 Jun 91 & 13 & 20.5400 & 6.3800 & 7.0800 \\
\hline 26 Jun 91 & 14 & 20.3900 & 6.3150 & 7.0900 \\
\hline 26 Jun 91 & 15 & 20.3800 & 6.3300 & 7.1150 \\
\hline 26 Jun 91 & 16 & 20.4033 & 6.2567 & 7.0433 \\
\hline 26 Jun 91 & 17 & 20.7600 & 6.4850 & 6.8450 \\
\hline 26 Jun 91 & 18 & 21.0450 & 6.6550 & 6.8650 \\
\hline 26 Jun 91 & 19 & 21.4300 & 6.7200 & 7.0100 \\
\hline 26 Jun 91 & 20 & 20.5100 & 6.2300 & 6.9350 \\
\hline 26 Jun 91 & 21 & 20.5350 & 6.2400 & 7.0100 \\
\hline 26 Jun 91 & 22 & 20.7400 & 6.3550 & 7.1150 \\
\hline 26 Jun 91 & 23 & 21.0950 & 6.4950 & 7.0850 \\
\hline 27 Jun 91 & 1 & 20.5900 & 6.2800 & 6.9900 \\
\hline 27 Jun 91 & 3 & 20.2050 & 6.2650 & 7.1000 \\
\hline 27 Jun 91 & 4 & 20.1300 & 6.2600 & 7.0900 \\
\hline 27 Jun 91 & 5 & 20.1700 & 6.2850 & 7.0500 \\
\hline 27 Jun 91 & 6 & 20.9750 & 6.4650 & 6.9600 \\
\hline 27 Jun 91 & 7 & 21.5900 & 6.6550 & 6.7200 \\
\hline 27 Jun 91 & 8 & 21.2300 & 6.6150 & 6.5800 \\
\hline 27 Jun 91 & 9 & 21.1700 & 6.5750 & 6.6300 \\
\hline 27 Jun 91 & 10 & 20.6550 & 6.4450 & 6.7850 \\
\hline 27 Jun 91 & 11 & 20.1850 & 6.3400 & 6.9650 \\
\hline
\end{tabular}


C. 12

Table C.1 (continued)

\begin{tabular}{|c|c|c|c|c|}
\hline Date & Hour & $\begin{array}{l}\text { Temperature } \\
\left({ }^{\circ} \mathrm{C}\right)\end{array}$ & pH & $\begin{array}{l}\text { Dissolved } \\
\text { axygen } \\
(\mathrm{mg} / \mathrm{L})\end{array}$ \\
\hline 27 Jun 91 & 12 & 20.1500 & 6.3850 & 6.9700 \\
\hline 27 Jun 91 & 13 & 20.6500 & 6.5350 & 6.6350 \\
\hline 27 Jun 91 & 14 & 20.8900 & 6.6100 & 6.8300 \\
\hline 27 Jun 91 & 15 & 21.1950 & 6.6550 & 7.0250 \\
\hline 27 Jun 91 & 16 & 21.2650 & 6.6600 & 7.1250 \\
\hline 27 Jun 91 & 17 & 21.2100 & 6.6600 & 7.1700 \\
\hline 27 Jun 91 & 18 & 21.3100 & 6.5400 & 7.2500 \\
\hline 27 Jun 91 & 19 & 21.3250 & 6.3900 & 7.2900 \\
\hline 27 Jun 91 & 20 & 21.3050 & 6.4450 & 7.3750 \\
\hline 27 Jun 91 & 21 & 21.2050 & 6.3850 & 7.3700 \\
\hline 27 Jun 91 & 22 & 21.3150 & 6.6650 & 7.4050 \\
\hline 27 Jun 91 & 23 & 21.3950 & 6.7050 & 7.4100 \\
\hline 28 Jun 91 & 0 & 21.3050 & 6.5200 & 7.2500 \\
\hline 28 Jun 91 & 1 & 21.1150 & 6.3350 & 7.0950 \\
\hline 28 Jun 91 & 2 & 21.0950 & 6.3400 & 7.1000 \\
\hline 28 Jun 91 & 3 & 21.0400 & 6.3400 & 7.0000 \\
\hline 28 Jun 91 & 4 & 21.1100 & 6.3900 & 7.0350 \\
\hline 28 Jun 91 & 5 & 21.1600 & 6.4450 & 7.0300 \\
\hline 28 Jun 91 & 6 & 21.1100 & 6.4000 & 6.9750 \\
\hline 28 Jun 91 & 7 & 21.1150 & 6.3850 & 6.9000 \\
\hline 28 Jun 91 & 8 & 21.0500 & 6.3450 & 6.7800 \\
\hline 28 Jun 91 & 9 & 21.0250 & 6.3200 & 6.7350 \\
\hline 28 Jun 91 & 10 & 21.0400 & 6.3250 & 6.7300 \\
\hline 28 Jun 91 & 11 & 21.5300 & 6.5850 & 7.2600 \\
\hline 28 Jun 91 & 12 & 21.4800 & 6.5050 & 7.1250 \\
\hline 28 Jun 91 & 13 & 21.5250 & 6.6200 & 7.3350 \\
\hline 28 Jun 91 & 14 & 21.5850 & 6.6200 & 7.3250 \\
\hline 28 Jun 91 & 15 & 20.6300 & 6.5000 & 7.2200 \\
\hline 28 Jun 91 & 17 & 20.6500 & 6.2350 & 7.4300 \\
\hline 28 Jun 91 & 18 & 20.4300 & 6.2300 & 7.4900 \\
\hline 28 Jun 91 & 19 & 20.3700 & 6.2400 & 7.5100 \\
\hline 28 Jun 91 & 20 & 21.4800 & 6.3500 & 7.5350 \\
\hline
\end{tabular}




\section{C-13}

Table C.1 (continued)

\begin{tabular}{|c|c|c|c|c|}
\hline Date & Hour & $\begin{array}{c}\text { Temperature } \\
\left({ }^{\circ} \mathrm{C}\right)\end{array}$ & $\mathrm{pH}$ & $\begin{array}{c}\text { Dissolved } \\
\text { axygen } \\
(\mathrm{mg} / \mathrm{L})\end{array}$ \\
\hline 28 Jun 91 & 21 & 20.0650 & 6.3550 & 7.4650 \\
\hline 28 Jun 91 & 22 & 20.0300 & 6.3450 & 7.3900 \\
\hline 28 Jun 91 & 23 & 19.9950 & 6.3750 & 7.2900 \\
\hline 29 Jun 91 & 0 & 19.9950 & 6.3800 & 7.1950 \\
\hline 29 Jun 91 & 1 & 20.0000 & 6.3850 & 7.1850 \\
\hline 29 Jun 91 & 2 & 19.9950 & 6.3750 & 7.1250 \\
\hline 29 Jun 91 & 3 & 19.9900 & 6.3600 & 7.0600 \\
\hline 29 Jun 91 & 4 & 19.9900 & 6.3650 & 6.9950 \\
\hline 29 Jun 91 & 5 & 19.9900 & 6.3800 & 6.9700 \\
\hline 29 Jun 91 & 6 & 19.9850 & 6.3100 & 6.9350 \\
\hline 29 Jun 91 & 7 & 19.9800 & 6.3750 & 6.9500 \\
\hline 29 Jun 91 & 8 & 19.9800 & 6.3500 & 6.9750 \\
\hline 29 Jun 91 & 9 & 19.9850 & 6.2400 & 6.9350 \\
\hline 29 Jun 91 & 10 & 19.9950 & 6.3700 & 7.1600 \\
\hline 29 Jun 91 & 11 & 20.0250 & 6.3650 & 7.0200 \\
\hline 29 Jun 91 & 12 & 20.0050 & 6.3650 & 6.9900 \\
\hline 29 Jun 91 & 13 & 20.0150 & 6.3750 & 7.0600 \\
\hline 29 Jun 91 & 14 & 20.0500 & 6.3850 & 7.1150 \\
\hline 29 Jun 91 & 15 & 20.7550 & 6.3900 & 7.3450 \\
\hline 29 Jun 91 & 16 & 20.3900 & 6.3950 & 7.3650 \\
\hline 29 Jun 91 & 17 & 19.2800 & 6.3850 & 7.5550 \\
\hline 29 Jun 91 & 18 & 19.2250 & 6.4150 & 7.5750 \\
\hline 29 Jun 91 & 19 & 20.0600 & 6.4450 & 7.4950 \\
\hline 29 Jun 91 & 20 & 20.7150 & 6.5000 & 7.5900 \\
\hline 29 Jun 91 & 21 & 19.5650 & 6.5050 & 7.8100 \\
\hline 29 Jun 91 & 22 & 19.5700 & 6.4800 & 7.7150 \\
\hline 29 Jun 91 & 23 & 19.5400 & 6.4700 & 7.6900 \\
\hline 30 Jun 91 & o & 19.0638 & 6.4900 & 7.8313 \\
\hline 30 Jun 91 & 1 & 19.0450 & 5.4788 & 7.7900 \\
\hline 30 Jun 91 & 2 & 19.0125 & 6.4738 & 7.7700 \\
\hline 30 Jun 91 & 3 & 18.9963 & 6.4538 & 7.7063 \\
\hline 30 Jun 91 & 4 & 19.0038 & 6.4488 & 7.6825 \\
\hline
\end{tabular}




\section{C-14}

Table C.1 (continued)

\begin{tabular}{|c|c|c|c|c|}
\hline Date & Hour & $\begin{array}{c}\text { Temperature } \\
\left({ }^{\circ} \mathrm{C}\right)\end{array}$ & $\mathrm{pH}$ & $\begin{array}{c}\text { Dissolved } \\
\text { oxygen } \\
(\mathrm{mg} / \mathrm{L})\end{array}$ \\
\hline 30 Jun 91 & 5 & 18.9744 & 6.4444 & 7.6278 \\
\hline 30 Jun 91 & 6 & 18.9978 & 6.4400 & 7.6611 \\
\hline 30 Jun 91 & 7 & 19.0150 & 6.4288 & 7.5875 \\
\hline 30 Jun 91 & 8 & 18.9963 & 6.4463 & 7.5875 \\
\hline 30 Jun 91 & 9 & 18.9744 & 6.3811 & 7.5722 \\
\hline 30 Jun 91 & 10 & 18.9938 & 6.3800 & 7.6013 \\
\hline 30 Jun 91 & 11 & 19.1688 & 6.3850 & 7.6513 \\
\hline 30 Jun 91 & 12 & 19.1550 & 6.3588 & 7.8800 \\
\hline 30 Jun 91 & 13 & 19.2288 & 6.3975 & 7.9375 \\
\hline 30 Jun 91 & 14 & 19.2925 & 6.4313 & 7.9250 \\
\hline 30 Jun 91 & 15 & 19.1633 & 6.4122 & 7.8900 \\
\hline 30 Jun 91 & 16 & 18.8090 & 6.4400 & 8.0350 \\
\hline 30 Jun 91 & 17 & 18.7263 & 6.4588 & 8.1363 \\
\hline 30 Jun 91 & 18 & 19.2300 & 6.4700 & 8.0738 \\
\hline 30 Jun 91 & 19 & 20.1450 & 6.5250 & 8.0275 \\
\hline 30 Jun 91 & 20 & 20.7363 & 6.5200 & 7.8588 \\
\hline 30 Jun 91 & 21 & 18.9538 & 6.4900 & 8.1275 \\
\hline 30 Jun 91 & 22 & 18.7450 & 6.4888 & 8.1163 \\
\hline 30 Jun 91 & 23 & 18.7500 & 6.4838 & 8.0613 \\
\hline 04 Jul 91 & 0 & 18.4975 & 6.4875 & 8.4100 \\
\hline 04 Jul 91 & 1 & 18.4975 & 6.5025 & 8.3675 \\
\hline $04 \mathrm{Jul} 91$ & 2 & 18.4875 & 6.5050 & 8.3100 \\
\hline 04 Jul 91 & 3 & 18.4600 & 6.5000 & 8.3225 \\
\hline 04 Jul 91 & 4 & 18.4450 & 6.4925 & 8.2975 \\
\hline 04 Jul 91 & 5 & 18.4600 & 6.4950 & 8.2875 \\
\hline 04 Jul 91 & 6 & 18.4600 & 6.5000 & 8.2775 \\
\hline 04 Jul 91 & 7 & 18.4625 & 6.4900 & 8.2525 \\
\hline $04 \mathrm{Jul} 91$ & 8 & 18.4650 & 6.5025 & 8.2050 \\
\hline $04 \mathrm{Jul} 91$ & 9 & 18.5275 & 6.4750 & 8.1750 \\
\hline 04 Jul 91 & 10 & 18.6000 & 6.4620 & 8.1320 \\
\hline 04 Jul 91 & 11 & 18.5200 & 6.4725 & 8.2475 \\
\hline
\end{tabular}




\section{C-15}

Table C.1 (continued)

\begin{tabular}{|c|c|c|c|c|}
\hline Date & Hour & $\begin{array}{c}\text { Temperature } \\
\left({ }^{\circ} \mathrm{C}\right)\end{array}$ & $\mathrm{pH}$ & $\begin{array}{c}\text { Dissolved } \\
\text { axygen } \\
(\mathrm{mg} / \mathrm{L})\end{array}$ \\
\hline 04 Jul 91 & 12 & 18.6650 & 6.4575 & 8.2950 \\
\hline 04 Jul 91 & 13 & 18.5900 & 6.4500 & 8.4025 \\
\hline $04 \mathrm{Jul} 91$ & 14 & 18.6750 & 6.4775 & 8.4825 \\
\hline 04 Jul 91 & 15 & 18.5625 & 6.4825 & 8.4850 \\
\hline $04 \mathrm{Jul} 91$ & 16 & 18.7125 & 6.5225 & 8.6050 \\
\hline 04 Jul 91 & 17 & 18.8575 & 6.5625 & 8.7600 \\
\hline 04 Jul 91 & 18 & 19.0150 & 6.5875 & 8.8850 \\
\hline 04 Jul 91 & 19 & 20.1100 & 6.5550 & 8.4850 \\
\hline O4 Jul 91 & 20 & 21.0667 & 6.5667 & 8.4400 \\
\hline 04 Jul 91 & 21 & 19.2650 & 6.5700 & 8.6250 \\
\hline 04 Jul 91 & 22 & 19.3900 & 6.4650 & 8.4100 \\
\hline 04 Jul 91 & 23 & 18.5333 & 6.5100 & 8.4267 \\
\hline $05 \mathrm{Jul} 91$ & 20 & 19.1700 & 6.6200 & 9.0900 \\
\hline $05 \mathrm{Jul} 91$ & 21 & 19.2000 & 6.6350 & 9.1300 \\
\hline 05 Jul 91 & 22 & 19.1900 & 6.6200 & 9.1000 \\
\hline U5 Jul 91 & 23 & 19.2200 & 6.6350 & 9.0700 \\
\hline 06 Jul 91 & 0 & 19.2450 & 6.6200 & 8.9900 \\
\hline 06 Jul 91 & 1 & 19.2150 & 6.6000 & 8.9650 \\
\hline $06 \mathrm{Jul} 91$ & 2 & 19.1900 & 6.6100 & 8.9850 \\
\hline $06 \mathrm{Jul} 91$ & 3 & 19.2050 & 6.6250 & 9.0100 \\
\hline $06 \mathrm{Jul} 91$ & 4 & 19.2200 & 6.6250 & 8.9900 \\
\hline O6 Jul 91 & 5 & 19.2300 & 6.6050 & 8.8950 \\
\hline $06 \mathrm{Jul} 91$ & 6 & 19.2200 & 6.5900 & 8.8550 \\
\hline 06 Jul 91 & 7 & 19.2150 & 6.5950 & 8.8300 \\
\hline 06 Jul 91 & 8 & 19.2200 & 6.5750 & 8.8400 \\
\hline $06 \mathrm{Jul} 91$ & 9 & 19.2050 & 6.6050 & 8.8750 \\
\hline $06 \mathrm{Jul} 91$ & 10 & 19.2200 & 6.6100 & 8.9750 \\
\hline $06 \mathrm{Jul} 91$ & 11 & 19.2250 & 6.6300 & 9.0000 \\
\hline $06 \mathrm{Jul} 91$ & 12 & 19.3600 & 6.5850 & 8.9350 \\
\hline $06 \mathrm{Jul} 91$ & 13 & 19.7150 & 6.5800 & 8.8600 \\
\hline $06 \mathrm{Jul} 91$ & 14 & 19.4600 & 6.5450 & 8.9300 \\
\hline $06 \mathrm{Jul} 91$ & 15 & 19.1900 & 6.5050 & 8.7900 \\
\hline
\end{tabular}


C-16

Table C.1 (continued)

\begin{tabular}{|c|c|c|c|c|}
\hline Date & Hour & $\begin{array}{c}\text { Temperature } \\
\left({ }^{\circ} \mathrm{C}\right)\end{array}$ & $\mathrm{pH}$ & $\begin{array}{c}\text { Dissolved } \\
\text { axygen } \\
(\mathrm{mg} / \mathrm{L})\end{array}$ \\
\hline $06 \mathrm{Jul} 91$ & 16 & 18.5950 & 6.4650 & 8.6200 \\
\hline $06 \mathrm{Jul} 91$ & 17 & 18.7700 & 6.5050 & 8.8050 \\
\hline $06 \mathrm{Jul} 91$ & 18 & 19.2100 & 6.6000 & 9.0300 \\
\hline $06 \mathrm{Jul} 91$ & 19 & 20.1250 & 6.6250 & 8.9600 \\
\hline $06 \mathrm{Jul} 91$ & 20 & 19.6200 & 6.6050 & 8.9850 \\
\hline $06 \mathrm{Jul} 91$ & 21 & 19.1650 & 6.5500 & 8.8550 \\
\hline $06 \mathrm{Jul} 91$ & 22. & 19.0600 & 6.5200 & 8.6750 \\
\hline $06 \mathrm{Jul} 91$ & 23 & 18.8550 & 6.4950 & 8.6600 \\
\hline 07 Jul 91 & 0 & 18.9300 & 6.5200 & 8.5725 \\
\hline 07 Jul 91 & 1 & 18.9375 & 6.5300 & 8.5550 \\
\hline 07 Jul 91 & 2 & 18.9475 & 6.5325 & 8.5125 \\
\hline 07 Jul 91 & 3 & 18.9150 & 6.5150 & 8.4575 \\
\hline $07 \mathrm{Jul} 91$ & 4 & 18.9360 & 6.5100 & 8.4440 \\
\hline 07 Jul 91 & 5 & 18.9100 & 6.5025 & 8.4300 \\
\hline 07 Jul 91 & 6 & 18.9250 & 6.5150 & 8.3975 \\
\hline 07 Jul 91 & 7 & 18.7700 & 6.4900 & 8.4400 \\
\hline 07 Jul 91 & 8 & 18.7700 & 6.5000 & 8.4250 \\
\hline 07 Jul 91 & 9 & 18.7950 & 6.4950 & 8.4150 \\
\hline 07 Jul 91 & 10 & 18.8200 & 6.5050 & 8.4150 \\
\hline 07 Jul 91 & 11 & 18.7900 & 6.5050 & 8.4050 \\
\hline 07 Jul 91 & 12 & 18.8250 & 6.5350 & 8.6750 \\
\hline 07 Jul 91 & 13 & 18.8650 & 6.5700 & 8.7200 \\
\hline 07 Jul 91 & 14 & 18.9300 & 6.5900 & 8.8300 \\
\hline 07 Jul 91 & 15 & 19.6300 & 6.5150 & 8.7700 \\
\hline 07 Jul 91 & 16 & 19.1500 & 6.5100 & 8.7700 \\
\hline 07 Jul 91 & 17 & 19.1750 & 6.5600 & 8.7200 \\
\hline 07 Jul 91 & 18 & 19.6700 & 6.5900 & 8.8300 \\
\hline $07 \mathrm{Jul} 91$ & 19 & 19.5500 & 6.6000 & 8.8850 \\
\hline 07 Jul 91 & 20 & 19.7250 & 6.6000 & 8.8200 \\
\hline 07 Jul 91 & 21 & 21.9750 & 6.6800 & 8.4900 \\
\hline $07 \mathrm{Jul} 91$ & 22 & 19.2000 & 6.5350 & 8.4800 \\
\hline 07 Jul 91 & 23 & 19.0950 & 6.5250 & 8.5100 \\
\hline
\end{tabular}




\section{C-17}

Table C.1 (continued)

\begin{tabular}{|c|c|c|c|c|}
\hline Date & Hour & $\begin{array}{l}\text { Temperature } \\
\left({ }^{\circ} \mathrm{C}\right)\end{array}$ & $\mathrm{pH}$ & $\begin{array}{c}\text { Dissolved } \\
\text { axygen } \\
(\mathrm{mg} / \mathrm{L})\end{array}$ \\
\hline $08 \mathrm{Jul} 91$ & 7 & 19.1000 & 6.5100 & 8.2500 \\
\hline 08 Jul 91 & 8 & 19.0500 & 6.5050 & 8.2750 \\
\hline $08 \mathrm{Jul} 91$ & 9 & 19.0400 & 6.5050 & 8.3200 \\
\hline $08 \mathrm{Jul} 91$ & 10 & 19.0650 & 6.5450 & 8.3900 \\
\hline 08 Jul 91 & 11 & 19.2000 & 6.5000 & 8.4100 \\
\hline $08 \mathrm{Jul} 91$ & 12 & 19.2800 & 6.5150 & 8.3750 \\
\hline $08 \mathrm{Jul} 91$ & 13 & 19.1250 & 6.4800 & 8.3650 \\
\hline $08 \mathrm{Jul} 91$ & 14 & 19.0150 & 6.4800 & 8.3450 \\
\hline $08 \mathrm{Jul} 91$ & 15 & 18.6650 & 6.4300 & 8.2500 \\
\hline $08 \mathrm{Jul} 91$ & 16 & 18.8150 & 6.5000 & 8.3900 \\
\hline 08 Jul 91 & 17 & 19.0700 & 6.5400 & 8.4900 \\
\hline $08 \mathrm{Jul} 91$ & 18 & 19.2250 & 6.5600 & 8.4900 \\
\hline $08 \mathrm{Jul} 91$ & 19 & 19.4650 & 6.5800 & 8.4750 \\
\hline $08 \mathrm{Jul} 91$ & 20 & 19.5700 & 6.6200 & 8.4700 \\
\hline 08 Jul 91 & 21 & 19.3600 & 6.5300 & 8.4600 \\
\hline 08 Jul 91 & 22 & 19.9600 & 6.6500 & 8.5200 \\
\hline $08 \mathrm{Jul} 91$ & 23 & 19.3600 & 6.5700 & 8.5000 \\
\hline 09 Jul 91 & 0 & 19.6280 & 6.6520 & 8.4900 \\
\hline $09 \mathrm{Jul} 91$ & 1 & 19.5683 & 6.6300 & 8.4650 \\
\hline 09 Jul 91 & 2 & 19.6200 & 6.6280 & 8.4380 \\
\hline 09 Jul 91 & 3 & 19.6350 & 6.6200 & 8.4050 \\
\hline 09 Jul 91 & 4 & 19.6540 & 6.6160 & 8.4000 \\
\hline 09 Jul 91 & 5 & 19.5950 & 6.6075 & 8.3525 \\
\hline 09 Jul 91 & 6 & 19.5850 & 6.5875 & 8.2775 \\
\hline $09 \mathrm{Jul} 91$ & 7 & 19.6600 & 6.6167 & 8.3000 \\
\hline \$9 Jul 91 & 8 & 19.8400 & 6.6300 & 8.3350 \\
\hline 09 Jul 91 & 9 & 19.8400 & 6.6500 & 8.4000 \\
\hline 09 Jul 91 & 10 & 19.8500 & 6.6400 & 8.3550 \\
\hline 09 Jul 91 & 11 & 19.8850 & 6.6500 & 8.3950 \\
\hline $09 \mathrm{Jul} 91$ & 12 & 19.4125 & 6.5600 & 8.2675 \\
\hline 09 Jul 91 & 13 & 19.6475 & 6.5675 & 8.3325 \\
\hline $09 \mathrm{Jul} 91$ & 14 & 19.2075 & 6.5250 & 8.2150 \\
\hline
\end{tabular}




\section{C-18}

Table C.1 (continued)

\begin{tabular}{|c|c|c|c|c|}
\hline Date & Hour & $\begin{array}{l}\text { Temperature } \\
\left({ }^{\circ} \mathrm{C}\right)\end{array}$ & $\mathrm{pH}$ & $\begin{array}{c}\text { Dissolved } \\
\text { axygen } \\
(\mathrm{mg} / \mathrm{L}) \\
\end{array}$ \\
\hline $09 \mathrm{Jul} 91$ & 15 & 19.3900 & 6.5800 & 8.2775 \\
\hline 09 Jul 91 & 16 & 19.5483 & 6.6033 & 8.3883 \\
\hline 09 Jul 91 & 17 & 19.6975 & 6.6450 & 8.5075 \\
\hline 09 Jul 91 & 18 & 19.8975 & 6.6850 & 8.6050 \\
\hline 09 Jul 91 & 19 & 19.6625 & 6.6600 & 8.5350 \\
\hline 09 Jul 91 & 20 & 19.9575 & 6.7000 & 8.5750 \\
\hline O9 Jul 91 & 21 & 21.9225 & 6.7875 & 8.6000 \\
\hline 09 Jul 91 & 22 & 20.8025 & 6.7150 & 8.6600 \\
\hline $09 \mathrm{Jul} 91$ & 23 & 19.6875 & 6.6675 & 8.5225 \\
\hline $10 \mathrm{Jul} 91$ & 0 & & & \\
\hline 11 Jul 91 & 0 & 19.2800 & 6.5850 & 8.1600 \\
\hline 11 Jul 91 & 1 & 19.2650 & 6.5900 & 8.1250 \\
\hline 11 Jul 91 & 2 & 19.3567 & 6.5833 & 8.1633 \\
\hline 11 Jul 91 & 3 & 19.3750 & 6.6075 & 8.1975 \\
\hline 11 Jul 91 & 4 & 19.3500 & 6.5940 & 8.1340 \\
\hline 11 Jul 91 & 5 & 19.3725 & 6.5875 & 8.1450 \\
\hline 11 Jul 91 & 6 & 19.3675 & 6.5700 & 8.1000 \\
\hline 11 Jul 91 & 7 & 19.3650 & 6.6050 & 8.1300 \\
\hline 11 Jul 91 & 8 & 19.3700 & 6.6050 & 8.1275 \\
\hline 11 Jul 91 & 9 & 19.3700 & 6.5975 & 8.1100 \\
\hline 11 Jul 91 & 10 & 19.3825 & 6.5800 & 8.1150 \\
\hline 11 Jul 91 & 11 & 19.5050 & 6.6275 & 8.2500 \\
\hline 11 Jul 91 & 12 & 19.5275 & 6.5825 & 8.2075 \\
\hline 11 Jul 91 & 13 & 19.2625 & 6.5600 & 8.1675 \\
\hline 11 Jul 91 & 14 & 19.0325 & 6.5425 & 8.0725 \\
\hline 11 Jul 91 & 15 & 19.2225 & 6.5825 & 8.1250 \\
\hline 11 Jul 91 & 16 & 19.2460 & 6.5820 & 8.1440 \\
\hline 11 Jul 91 & 17 & 19.3750 & 6.6050 & 8.2325 \\
\hline 11 Jul 91 & 18 & 19.3200 & 6.6075 & 8.2325 \\
\hline $11 \mathrm{Jul} 91$ & 19 & 19.4525 & 6.6225 & 8.2275 \\
\hline 11 Jul 91 & 20 & 19.7625 & 6.6225 & 8.2725 \\
\hline 11 Jul 91 & 21 & 19.6975 & 6.6500 & 8.1475 \\
\hline
\end{tabular}


C-19

Table C.1 (continued)

\begin{tabular}{|c|c|c|c|c|}
\hline Date & Hour & $\begin{array}{c}\text { Temperature } \\
\left({ }^{\circ} \mathrm{C}\right)\end{array}$ & $\mathrm{pH}$ & $\begin{array}{l}\text { Dissolved } \\
\text { axygen } \\
(\mathrm{mg} / \mathrm{L})\end{array}$ \\
\hline $11 \mathrm{Jul} 91$ & 22 & 20.5625 & 6.6575 & 8.0375 \\
\hline 11 Jul 91 & 23 & 18.8700 & 6.5550 & 8.0725 \\
\hline $12 \mathrm{Jul} 91$ & 0 & & & \\
\hline 13 Jul 91 & 0 & 18.5000 & 6.5125 & 7.9075 \\
\hline $13 \mathrm{Jul} 91$ & 1 & 18.5375 & 6.5125 & 7.8875 \\
\hline 13 Jul 91 & 2 & 18.5525 & 6.5050 & 7.8425 \\
\hline 13 Jul 91 & 3 & 18.4700 & 6.4975 & 7.8000 \\
\hline 13 Jul 91 & 4 & 18.4800 & 6.5050 & 7.8075 \\
\hline 13 Jul 91 & 5 & 18.4700 & 6.5000 & 7.7675 \\
\hline 13 Jul 91 & 6 & 18.4850 & 6.5000 & 7.7325 \\
\hline 13 Jul 91 & 7 & 18.4975 & 6.4950 & 7.7275 \\
\hline 13 Jul 91 & 8 & 18.4625 & 6.5025 & 7.7225 \\
\hline 13 Jul 91 & 9 & 18.4880 & 6.5060 & 7.7720 \\
\hline 13 Jul 91 & 10 & 18.5300 & 6.5175 & 7.8050 \\
\hline 13 Jul 91 & 11 & 18.5400 & 6.5200 & 7.8075 \\
\hline 13 Jul 91 & 12 & 18.7525 & 6.5175 & 7.8200 \\
\hline 13 Jul 91 & 13 & 18.7400 & 6.5150 & 7.8375 \\
\hline 13 Jul 91 & 14 & 18.8775 & 6.5225 & 7.9500 \\
\hline 13 Jul 91 & 15 & 19.1375 & 6.5275 & 7.8575 \\
\hline 13 Jul 91 & 16 & 18.8600 & 6.5200 & 7.8900 \\
\hline 13 Jul 91 & 17 & 18.7325 & 6.5400 & 7.8725 \\
\hline 13 Jul 91 & 18 & 18.8275 & 6.5525 & 7.9450 \\
\hline $13 \mathrm{Jul} 91$ & 19 & 19.1825 & 6.5700 & 8.0600 \\
\hline 13 Jul 91 & 20 & 19.1450 & 6.5975 & 8.0475 \\
\hline 13 Jul 91 & 21 & 19.7220 & 6.6380 & 8.1220 \\
\hline 13 Jul 91 & 22 & 19.7150 & 6.5800 & 7.8650 \\
\hline $13 \mathrm{Jul} 91$ & 23 & 18.2975 & 6.5050 & 7.8325 \\
\hline 14 Jul 91 & 0 & & & \\
\hline $15 \mathrm{Jul} 91^{\circ}$ & 0 & 18.0350 & 6.4950 & 1.7350 \\
\hline $15 \mathrm{Jul} 91$ & 1 & 18.0500 & 6.4750 & 7.7050 \\
\hline 15 Jul 91 & 2 & 18.0650 & 6.4850 & 7.6800 \\
\hline 15 Jul 91 & 3 & 18.0300 & 6.4800 & 7.6250 \\
\hline
\end{tabular}


Table C.1 (continued)

\begin{tabular}{|c|c|c|c|c|}
\hline Date & Hour & $\begin{array}{l}\text { Temperature } \\
\left({ }^{\circ} \mathrm{C}\right)\end{array}$ & $\mathrm{pH}$ & $\begin{array}{l}\text { Dissolved } \\
\text { axygen } \\
(\mathrm{mg} / \mathrm{L})\end{array}$ \\
\hline 15 Jul 91 & 4 & 18.0300 & 6.4700 & 7.5950 \\
\hline 15 Jul 91 & 5 & 18.0250 & 6.4850 & 7.6450 \\
\hline 15 Jul 91 & 6 & 18.0300 & 6.4900 & 7.6100 \\
\hline 15 Jul 91 & 7 & 18.0250 & 6.4750 & 7.6000 \\
\hline 15 Jul 91 & 8 & 18.0350 & 6.4750 & 7.5350 \\
\hline 15 Jul 91 & 9 & 18.0300 & 6.4500 & 7.5100 \\
\hline 15 Jul 91 & 10 & 18.0400 & 6.4800 & 7.5800 \\
\hline 15 Jul 91 & 11 & 18.3150 & 6.4850 & 7.6800 \\
\hline 15 Jul 91 & 12 & 18.9500 & 6.5000 & 8.1100 \\
\hline 15 Jul 91 & 13 & 18.9300 & 6.5350 & 7.8150 \\
\hline $15 \mathrm{Jul} 91$ & 14 & 18.9350 & 6.5400 & 7.5800 \\
\hline 15 Jul 91 & 15 & 19.3550 & 6.6200 & 7.6300 \\
\hline 15 Jul 91 & 16 & 19.4950 & 6.6550 & 7.6100 \\
\hline 15 Jul 91 & 17 & 19.7600 & 6.6900 & 7.6850 \\
\hline 15 Jul 91 & 18 & $19 . \%$ & 6.7050 & 7.6700 \\
\hline 15 Jul 91 & 19 & 20.1300 & 6.7500 & 7.7000 \\
\hline 15 Jul 91 & 21 & 20.5250 & 6.7900 & 7.7450 \\
\hline 15 Jul 91 & 22 & 19.4600 & 6.6850 & 7.5600 \\
\hline 15 Jul 91 & 23 & 19.4400 & 6.6750 & 7.5400 \\
\hline $16 \mathrm{Jul} 91$ & 0 & 19.4550 & 6.6750 & 7.5350 \\
\hline $16 \mathrm{Jul} 91$ & 1 & 19.4800 & 6.6750 & 7.5200 \\
\hline 16 Jul 91 & 2 & 19.4550 & 6.6650 & 7.4350 \\
\hline 16 Jul 91 & 3 & 19.4300 & 6.6650 & 7.4200 \\
\hline 16 Jul 91 & 4 & 19.4067 & 6.6067 & 7.3767 \\
\hline 16 Jul 91 & 5 & 19.4050 & 6.5200 & 7.3200 \\
\hline $16 \mathrm{Jul} 91$ & 6 & 19.3900 & 6.6000 & 7.2850 \\
\hline 16 Jul 91 & 7 & 19.3950 & 6.6350 & 7.2050 \\
\hline $10 \div .191$ & 8 & 19.2950 & 6.6200 & 7.1100 \\
\hline 16 Jul 91 & 9 & 19.3400 & 6.5200 & 7.1200 \\
\hline $16 \mathrm{Jul} 91$ & 10 & 19.3650 & 6.6150 & 7.2050 \\
\hline $16 \mathrm{Jul} 91$ & 11 & 19.6100 & 6.5950 & 7.2700 \\
\hline 16 Jul 91 & 12 & 19.2750 & 6.5700 & 7.1750 \\
\hline
\end{tabular}


Table C.1 (continued)

\begin{tabular}{|c|c|c|c|c|}
\hline Date & Hour & $\begin{array}{c}\text { Tem ature } \\
\text { (ic) }\end{array}$ & pH & $\begin{array}{c}\text { Dissolved } \\
\text { oxygen } \\
\text { (mg/L) }\end{array}$ \\
\hline 16 Jul 91 & 13 & 18.9100 & 6.5600 & 7.1200 \\
\hline 17 Jul 91 & 16 & 19.1650 & 6.6050 & 6.8750 \\
\hline 17 Jul 91 & 17 & 19.4650 & 6.6750 & 7.0900 \\
\hline 17 Jul 91 & 18 & 19.2400 & 6.6500 & 6.9850 \\
\hline 17 Jul 91 & 19 & 19.3850 & 6.7000 & 7.0950 \\
\hline 17 Jul 91 & 20 & 19.3850 & 6.6900 & 7.1250 \\
\hline 17 Jul 91 & 21 & 20.4650 & 6.8500 & 7.5450 \\
\hline 17 Jul 91 & 22 & 21.1350 & 6.8700 & 7.5450 \\
\hline 17 Jul 91 & 23 & 19.2300 & 6.6500 & 6.9600 \\
\hline 18 Jul 91 & 0 & & & \\
\hline $20 \mathrm{Jul} 91$ & 0 & 18.9440 & 6.5880 & 6.7400 \\
\hline 20 Jul 91 & 1 & 19.0150 & 6.5925 & 6.7600 \\
\hline $20 \mathrm{Jul} 91$ & 2 & 19.0100 & 6.5825 & $6.7 i 50$ \\
\hline $20 \mathrm{Jul} 91$ & 3 & 18.9900 & 6.5575 & 6.6950 \\
\hline 20 Jul 91 & 4 & 18.9875 & 6.5400 & 6.6800 \\
\hline 20 Jul 91 & 5 & 18.9925 & 6.5100 & 6.6275 \\
\hline $20 \mathrm{Jul} 91$ & 6 & 18.9875 & 6.5400 & 6.6175 \\
\hline $20 \mathrm{Jul} 91$ & 7 & 18.5875 & 6.4725 & 6.6350 \\
\hline 20 Jul 91 & 8 & 18.9775 & 6.5100 & 6.5650 \\
\hline $20 \mathrm{Jul} 91$ & 9 & 19.0025 & 6.5625 & 6.5925 \\
\hline 20 Jul 91 & 10 & 19.0400 & 6.4950 & 6.6275 \\
\hline $20 \mathrm{Jul} 91$ & 11 & 19.0825 & 6.4975 & 6.7650 \\
\hline 20 Jul 91 & 12 & 19.3000 & 6.5625 & 6.8175 \\
\hline $20 \mathrm{Jul} 91$ & 13 & 19.2560 & 6.5480 & 6.8180 \\
\hline $20 \mathrm{Jul} 91$ & 14 & 19.2750 & 6.5175 & 6.8225 \\
\hline $20 \mathrm{Jul} 91$ & 15 & 19.0750 & 6.5300 & 6.7525 \\
\hline 20 Jul 91 & 16 & 18.9550 & 6.4925 & 6.6875 \\
\hline $20 \mathrm{Jul} 91$ & 17 & 19.0125 & 6.5175 & 6.7175 \\
\hline $20 \mathrm{Jul} 91$ & 18 & 19.4375 & 6.6175 & 7.0100 \\
\hline 20 Jul 91 & 19 & 19.7775 & 6.6800 & 7.1625 \\
\hline $20 \mathrm{Jul} 91$ & 20 & 19.8500 & 6.6900 & 7.1325 \\
\hline $20 \mathrm{Jul} 91$ & 21 & 19.4350 & 6.6600 & 6.9150 \\
\hline
\end{tabular}




\section{C-22}

Table C.1 (continued)

\begin{tabular}{|c|c|c|c|c|}
\hline Date & Hour & $\begin{array}{c}\text { Temperature } \\
\left({ }^{\circ} \mathrm{C}\right)\end{array}$ & $\mathrm{pH}$ & $\begin{array}{c}\text { Dissolved } \\
\text { oxygen } \\
(m g / L)\end{array}$ \\
\hline $20 \mathrm{Jul} 91$ & 22 & 18.88 .25 & 6.5650 & 6.7375 \\
\hline $20 \mathrm{Jul} 91$ & 23 & 18.8800 & 6.5575 & 6.6950 \\
\hline 21 Jul 9: & 0 & & & \\
\hline 22 Jul 91 & $\mathbf{0}$ & 18.8625 & 6.5725 & 6.7575 \\
\hline 22 Jul 91 & 1 & 18.9275 & 6.6000 & 6.7550 \\
\hline 22 Jul 91 & 2 & 18.8975 & 6.5875 & 67025 \\
\hline 22 Jul 91 & 3 & 18.8750 & 6.5850 & 6.6700 \\
\hline 22 Jul 91 & 4 & 18.8840 & 6.5940 & 6.6800 \\
\hline 22 Jul 91 & 5 & 18.8725 & 6.5775 & 6.6375 \\
\hline 22 Jul 91 & 6 & 18.8675 & 6.5700 & 6.6300 \\
\hline 22 Jul 91 & 7 & 18.8550 & 6.5625 & 6.6200 \\
\hline $22 \mathrm{Ju}, 91$ & 8 & 18.8850 & 6.5800 & 6.6000 \\
\hline 22 Jul 91 & 9 & 18.9000 & 6.5350 & 6.6600 \\
\hline 22 Jul 91 & 10 & 18.9825 & 6.5025 & 6.6850 \\
\hline 22 Jul 91 & 11 & 18.6900 & 6.3950 & 6.7250 \\
\hline 22 Jul 91 & 12 & 19.0125 & 6.4500 & 6.8075 \\
\hline 22 Jul 91 & 13 & 19.0750 & 6.5650 & 6.7875 \\
\hline 22 Jul 91 & 14 & 18.9600 & 6.56 .50 & 6.7225 \\
\hline 22 Jul 91 & 15 & 19.1175 & 6.5900 & 6.8275 \\
\hline $22 \mathrm{Jul} 91$ & 16 & 19.1420 & 5.8780 & 7.5280 \\
\hline $22 \mathrm{Jul} 91$ & 17 & 19.3200 & 5.7700 & 7.8325 \\
\hline 22 Jul 91 & 18 & 19.2575 & 5.7875 & 7.8675 \\
\hline 22 Jul 91 & 19 & 19.7900 & 5.8700 & 8.2625 \\
\hline 22. Sol 91 & 20 & 19.9825 & 5.9275 & 8.3350 \\
\hline $22 \operatorname{In} 91$ & 21 & 20.2475 & 5.9850 & 8.4925 \\
\hline 22. Jul 91 & 22 & 18.9075 & 5.7375 & 7.7375 \\
\hline 22. Jul 91 & 23 & 18.8250 & 5.7425 & 7.6825 \\
\hline 23 Jul 91 & 0 & & & \\
\hline $24 \mathrm{Jul} 91$ & 0 & 18.7600 & 4.8250 & 8.2500 \\
\hline $24 \mathrm{Jul} 91$ & 1 & 18.7750 & 4.8225 & 8.2450 \\
\hline $24 \mathrm{Jul} 91$ & 2 & 18.8225 & 4.8350 & 8.2100 \\
\hline 24 Jul 91 & 3 & 18.8325 & 4.8150 & 8.1650 \\
\hline
\end{tabular}


Table C.1 (continued)

\begin{tabular}{|c|c|c|c|c|}
\hline Date & Hour & $\begin{array}{c}\text { Temperature } \\
\left({ }^{\circ} \mathrm{C}\right)\end{array}$ & $\mathrm{pH}$ & $\begin{array}{c}\text { Dissolved } \\
\text { arygen } \\
\text { (mg/L) }\end{array}$ \\
\hline 24 Jul 91 & 4 & 18.8200 & 4.8050 & 8.1250 \\
\hline 24 Jul 91 & 5 & 18.7900 & 4.8025 & 8.0600 \\
\hline 24 Jul 91 & 6 & 18.7675 & 4.7950 & 8.0550 \\
\hline 24 Jul 91 & 7 & 18.7400 & 4.7925 & 8.0575 \\
\hline 24 Jul 91 & 8 & 18.7550 & 4.8075 & 8.0950 \\
\hline 24 Jul 91 & 9 & 18.7800 & 4.8060 & 8.0620 \\
\hline 24 Jul 91 & 10 & 18.8000 & 4.7875 & 8.0900 \\
\hline 24 Jul 91 & 11 & 18.5625 & 4.7700 & 8.0225 \\
\hline 24 Jul 91 & 12 & 18.7900 & 4.7950 & 8.1625 \\
\hline 24 Jul 91 & 13 & 18.8300 & 4.8150 & 8.1775 \\
\hline 24 Jul 91 & 14 & 18.6900 & 4.7975 & 8.1550 \\
\hline 24 Jul 91. & 15 & 18.7200 & 4.8100 & 8.1575 \\
\hline 24 Jul 91 & 16 & 18.7325 & 4.8350 & 8.2000 \\
\hline 24 Jul 91 & 17 & 18.9900 & 4.8550 & 8.2875 \\
\hline 24 Jul 91 & 18 & 19.0050 & 4.8550 & 8.2650 \\
\hline 24 Jul 91 & 19 & 19.2325 & 4.8975 & 8.2475 \\
\hline 24 Jul 91 & 20 & 19.1225 & 4.8675 & 8.1900 \\
\hline 24 Jul 91 & 21 & 19.5700 & 4.8980 & 8.2180 \\
\hline 24 Jul 91 & 22 & 19.4350 & 4.9050 & 8.0600 \\
\hline 24 Jul 91 & 23 & 19.8650 & 4.8950 & 7.9100 \\
\hline 25 Jul 91 & 0 & & & \\
\hline $26 \mathrm{Jul} 91$ & 0 & 18.7167 & 5.9533 & 7.2000 \\
\hline $26 \mathrm{Jul} 91$ & 1 & 18.6000 & 5.9483 & 7.2183 \\
\hline $26 \mathrm{Jul} 91$ & 2 & 18.6000 & 5.9500 & 7.1983 \\
\hline 26 Jul 91 & 3 & 18.5717 & 5.9383 & 7.1600 \\
\hline 26 Jul 91 & 4 & 18.5467 & 5.9350 & 7.1283 \\
\hline 26 Jul 91 & 5 & 18.6400 & 5.9350 & 7.0467 \\
\hline 26 Jul 91 & 6 & 18.5900 & 5.9267 & 7.0367 \\
\hline 26 Jul 91 & 7 & 18.5617 & 5.9300 & 7.0550 \\
\hline $26 \mathrm{Jul} 91$ & 8 & 18.5133 & 5.9250 & 7.0450 \\
\hline 26 Jul 91 & 9 & 18.5417 & 5.9300 & 7.0567 \\
\hline 26 Jul 91 & 10 & 18.5600 & 5.9300 & 7.0600 \\
\hline
\end{tabular}


Table C.1 (costinued)

\begin{tabular}{|c|c|c|c|c|}
\hline Date & Hour & $\begin{array}{c}\text { Temperature } \\
\left({ }^{\circ} \mathrm{C}\right)\end{array}$ & $\mathrm{pH}$ & $\begin{array}{l}\text { Dissolved } \\
\text { oxygen } \\
(\mathrm{mg} / \mathrm{L})\end{array}$ \\
\hline 26 Jul 91 & 11 & 18.6483 & 5.8950 & 7.1050 \\
\hline 26 Jul 91 & 12 & 18.7150 & 5.8850 & 7.1000 \\
\hline $26 \mathrm{Jul} 91$ & 13 & 18.5829 & 5.9643 & 7.0857 \\
\hline 26 Jul 91 & 14 & 18.6725 & 6.4250 & 6.7925 \\
\hline $26 \mathrm{Jul} 91$ & 15 & 18.5400 & 6.4317 & 7.0550 \\
\hline $26 \mathrm{Jul} 91$ & 16 & 18.621 .7 & 6.5050 & 6.9717 \\
\hline 26 Jul 91 & 17 & 18.6150 & 6.4700 & 6.9367 \\
\hline $26 \mathrm{Jul} 91$ & 18 & 18.8100 & 6.4800 & 6.9733 \\
\hline $26 \mathrm{Jul} 91$ & 19 & 18.7950 & 6.5033 & 6.9683 \\
\hline $26 \mathrm{Jul} 91$ & 20 & 18.6367 & 6.5217 & 6.8883 \\
\hline 26 Jul 91 & 21 & 18.5167 & 6.5283 & 6.8483 \\
\hline $26 \mathrm{Jul} 91$ & 22 & 18.5000 & 6.5300 & 6.8150 \\
\hline 26 Jul 91 & 23 & 19.5033 & 6.6000 & 6.9083 \\
\hline 27 Jul 91 & 0 & & & \\
\hline $29 \mathrm{Jul} 91$ & 0 & 17.4233 & 6.4567 & 6.3200 \\
\hline $29 \mathrm{Jul} 91$ & 1 & 17.4850 & 6.4525 & 6.3775 \\
\hline 29 Jul 91 & 2 & 17.5075 & 6.4525 & 6.3475 \\
\hline 29 Jul 91 & 3 & 17.5175 & 6.4500 & 6.3150 \\
\hline $29 \mathrm{Jul} 91$ & 4 & 17.4960 & 6.4440 & 6.2880 \\
\hline $29 \mathrm{Jul} 91$ & 5 & 17.4600 & 6.4225 & 6.2825 \\
\hline 29 Jul 91 & 6 & 17.4825 & 6.3800 & 6.2850 \\
\hline 29 Jul 91 & 8 & 17.4900 & 6.4275 & 6.2525 \\
\hline 29 Jul 91 & 9 & 17.5500 & 6.4400 & 6.2575 \\
\hline $29 \mathrm{Jul} 91$ & 10 & 17.5500 & 6.4400 & 6.1950 \\
\hline 29 Jul 91 & 11 & 17.4725 & 6.4425 & 6.2950 \\
\hline 29 Jul 91 & 12 & 17.5300 & 6.4550 & 6.3450 \\
\hline $29 \mathrm{Jul} 91$ & 13 & 17.7275 & 6.4775 & 6.3800 \\
\hline $29 \mathrm{Jul} 91$ & 14 & 17.7450 & 6.4900 & 6.4150 \\
\hline 29 Jul 91 & 15 & 17.7925 & 6.5000 & 6.4475 \\
\hline $29 \mathrm{Jul} 91$ & 16 & 17.8040 & 6.5220 & 6.5060 \\
\hline 29 Jul 91 & 17 & 17.7125 & 6.5275 & 6.5325 \\
\hline 29 Jul 91 & 18 & 17.8800 & 6.5525 & 6.6525 \\
\hline
\end{tabular}


Table C.1 (continued)

\begin{tabular}{|c|c|c|c|c|}
\hline Date & Hour & $\begin{array}{c}\text { Temperature } \\
\left({ }^{\circ} \mathrm{C}\right)\end{array}$ & pH & $\begin{array}{c}\text { Dissolved } \\
\text { arygen } \\
(\mathrm{mg} / \mathrm{L})\end{array}$ \\
\hline $29 \mathrm{Jul} 91$ & 19 & 18.3575 & 6.6100 & 6.7400 \\
\hline 29 Jul 91 & 20 & 18.3250 & 6.6200 & 6.7875 \\
\hline 29 Jul 91 & 21 & 19.5275 & 6.7075 & 6.8325 \\
\hline 29 Jul 91 & 22 & 17.6750 & 6.5225 & 6.5250 \\
\hline 29 Jul 91 & 23 & 17.6450 & 6.5225 & 6.5500 \\
\hline $30 \mathrm{Jul} 91$ & 0 & & & \\
\hline 31 Jul 91 & 0 & 17.6250 & 6.5350 & 6.5650 \\
\hline 31 Jul 91 & 1 & 17.6750 & 6.5350 & 6.5450 \\
\hline 31 Jul 91 & 2 & 17.6200 & 6.5250 & 6.4950 \\
\hline 31 Jul 91 & 3 & 17.6300 & 6.5150 & 6.4900 \\
\hline $31 \mathrm{Jul} 91$ & 4 & 17.6450 & 6.5150 & 6.4400 \\
\hline 31 Jul 91 & 5 & 17.5800 & 6.5100 & 6.3750 \\
\hline 31 Jul 91 & 6 & 17.5600 & 6.4550 & 6.4000 \\
\hline $31 \mathrm{Jul} \& 1$ & 7 & 17.8167 & 6.4733 & 6.3667 \\
\hline $31 \mathrm{Jul} 91$ & 8 & 17.9425 & 6.4850 & 6.3225 \\
\hline 31 Jul 91 & $i$ & 18.0600 & 6.4675 & 6.3525 \\
\hline 31 Jul 91 & 20 & 17.9900 & 6.4200 & 6.3750 \\
\hline 31 Jul 91 & 11 & 17.9650 & 6.4900 & 6.4650 \\
\hline 31 Jul 91 & 12 & 17.9175 & 6.4350 & 6.4175 \\
\hline $31 \mathrm{Jul} 91$ & 13 & 18.2450 & 6.5475 & 6.5075 \\
\hline 31 Jul 91 & 14 & 18.4325 & 6.4825 & 6.5750 \\
\hline $31 \mathrm{Jul} 91$ & 15 & 18.5675 & 6.4825 & 6.6400 \\
\hline 31 Jul 91 & 16 & 18.5240 & 6.5600 & 6.7040 \\
\hline 31 Jul 91 & 17 & 18.5100 & 6.5600 & 6.6500 \\
\hline 31 Jul 91 & 18 & 18.5975 & 6.5350 & 6.6750 \\
\hline 31 Jul 91 & 19 & 19.3375 & 6.5800 & 6.8375 \\
\hline 31 Jul 91 & 20 & 18.4775 & 6.5850 & 6.5725 \\
\hline $31 \mathrm{Jul} 91$ & 21 & 18.3025 & 6.5575 & 6.6375 \\
\hline 31 Jul 91 & 22 & 18.2900 & 6.4733 & 6.5633 \\
\hline 31 Jul 91 & 23 & 18.3800 & 6.3050 & 6.4250 \\
\hline 07 Aug 91 & 0 & 18.3850 & 6.4100 & 6.3700 \\
\hline
\end{tabular}




\section{C-26}

Table C.1 (continued)

\begin{tabular}{|c|c|c|c|c|}
\hline Date & Hour & $\begin{array}{c}\text { Temperature } \\
\left({ }^{\circ} \mathrm{C}\right)\end{array}$ & $\mathrm{pH}$ & $\begin{array}{l}\text { Dissolved } \\
\text { axygen } \\
(\mathrm{mg} / \mathrm{L})\end{array}$ \\
\hline 07 Aug 91 & 1 & 18.3250 & 6.4850 & 6.4200 \\
\hline 07 Aug 91 & 2 & 18.3250 & 6.3750 & 6.4050 \\
\hline C7 Aug 91 & 3 & 18.3400 & 6.4100 & 6.4400 \\
\hline 07 Aug 91 & 4 & 18.3267 & 6.3833 & 6.3700 \\
\hline 07 Aug 91 & 5 & 18.3400 & 6.3950 & 6.3700 \\
\hline 07 Aug 91 & 6 & 18.3550 & 6.4200 & 6.3400 \\
\hline 07 Aug 91 & 7 & 18.3400 & 6.4200 & 6.2550 \\
\hline 07 Aug 91 & 8 & 18.3300 & 6.4050 & 6.2600 \\
\hline 07 Aug 91 & 9 & 18.3100 & 6.2750 & 6.2950 \\
\hline 07 Aug 91 & 10 & 18.3150 & 6.3000 & 6.4350 \\
\hline 07 Aug 91 & 11 & 18.3800 & 6.3850 & 6.3700 \\
\hline 07 Auy 91 & 12 & $18.59 \mathrm{CO}$ & 6.4500 & 6.3950 \\
\hline 07 Aug 91 & 13 & 18.8100 & 6.3800 & 6.4700 \\
\hline 07 Aug 91 & 14 & 18.7200 & 6.3850 & 6.4900 \\
\hline Ji Aug 91 & 15 & 18.7550 & 6.3750 & 6.4750 \\
\hline 07 Aug 91 & 16 & 18.4100 & 6.4000 & 6.4300 \\
\hline 07 Aug 91 & 17 & 18.2600 & 6.3300 & 6.4250 \\
\hline 07 Aug 91 & 18 & 18.8750 & 6.6000 & 6.6000 \\
\hline $07 \mathrm{Au}_{\mathrm{b}} 91$ & 19 & 19.9850 & 6.6400 & 6.8300 \\
\hline 07 Aug 91 & 20 & 18.9600 & 6.5650 & 6.5700 \\
\hline 07 Aug 91 & 21 & 18.5250 & 6.5550 & 6.3900 \\
\hline 07 Aug 91 & 22 & 18.2850 & 6.4500 & 6.3650 \\
\hline 07 Aug 91 & 23 & 18.2150 & 6.3800 & 6.3350 \\
\hline 08 Aug 91 & 0 & 18.6850 & 6.5800 & 6.5600 \\
\hline 08 Aug 91 & 1 & 18.6800 & 6.5650 & 6.5200 \\
\hline 08 Aug 91 & 2 & 18.6750 & 6.5600 & 6.4900 \\
\hline 08 Aug 91 & 3 & 18.6600 & 6.5650 & 6.5150 \\
\hline 08 Aug 91 & 4 & 18.6600 & 6.5600 & 6.4800 \\
\hline 08 Aug 91 & 5 & 18.6600 & 6.5400 & 6.4550 \\
\hline 08 Aug 91 & 6 & 18.6500 & 6.5550 & 6.4450 \\
\hline 08 Aug 91 & 7 & 18.3875 & 6.4725 & 6.2725 \\
\hline 08 Aug 91 & 8 & 18.4075 & 6.4975 & 6.2600 \\
\hline
\end{tabular}


C-27

Table C.1 (continued)

\begin{tabular}{|c|c|c|c|c|}
\hline Date & Hour & $\begin{array}{c}\text { Temperature } \\
\left({ }^{\circ} \mathrm{C}\right)\end{array}$ & pH & $\begin{array}{c}\text { Dissolved } \\
\text { axygen } \\
(\mathrm{mg} / \mathrm{L})\end{array}$ \\
\hline 08 Aug 91 & 9 & 18.4225 & 6.4500 & 6.2850 \\
\hline 08 Aug 91 & 10 & 18.4400 & 6.4875 & 6.3325 \\
\hline 08 Aug 91 & 11 & 18.4625 & 6.4875 & 6.3775 \\
\hline 08 Aug 91 & 12 & 18.7675 & 6.5350 & 6.4350 \\
\hline 08 Aug 91 & 13 & 18.7450 & 6.4600 & 6.3875 \\
\hline 08 Aug 91 & 14 & 18.6800 & 6.4500 & 6.4175 \\
\hline 08 Aug 91 & 15 & 18.6525 & 6.4500 & 6.4000 \\
\hline 08 Aug 91 & 16 & 18.5700 & 6.3460 & 6.3340 \\
\hline 08 Aug 91 & 17 & 18.4775 & 6.4050 & 6.3075 \\
\hline 08 Aug 91 & 18 & 19.0575 & 6.4250 & 6.3925 \\
\hline 08 Aug 91 & 19 & 18.7725 & 6.4400 & 6.3525 \\
\hline 08 Aug 91 & 20 & 18.7075 & 6.4900 & 6.3650 \\
\hline 08 Aug 91 & 21 & 18.7250 & 6.5475 & 6.4075 \\
\hline 08 Aug 91 & 22 & 18.3850 & 6.5100 & 6.4100 \\
\hline 08 Aug 91 & 23 & 18.3950 & 6.5325 & 6.3775 \\
\hline 09 Aug 91 & 0 & & & \\
\hline 10 Aug 91 & 0 & 18.2875 & 6.4675 & 6.1175 \\
\hline 10 Aug 91 & 1 & 18.3125 & 6.4825 & 6.1050 \\
\hline 10 Aug 91 & 2 & 18.3000 & 6.4825 & 6.0900 \\
\hline 10 Aug 91 & 3 & 18.2850 & 6.4700 & 6.0800 \\
\hline 10 Aug 91 & 4 & 18.3000 & 6.4800 & 6.0825 \\
\hline 10 Aug 91 & 5 & 18.3000 & 6.4850 & 6.0500 \\
\hline 10 Aug 91 & 6 & 18.2800 & 6.4875 & 6.0250 \\
\hline 10 Aug 91 & 7 & 18.2800 & 6.4875 & 6.0250 \\
\hline 10 Aug 91 & 8 & 18.2750 & 6.4650 & 6.0275 \\
\hline 10 Aug 91 & 9 & 18.3500 & 6.3600 & 6.0360 \\
\hline 10 Aug 91 & 10 & 18.3175 & 6.3875 & 6.0075 \\
\hline 10 Aug 91 & 11 & 18.3325 & 6.3800 & 6.0300 \\
\hline 10 Aug 91 & 12 & 18.4150 & 6.3525 & 6.0300 \\
\hline 10 Aug 91 & 13 & 18.7050 & 6.3825 & 6.1325 \\
\hline 10 Aug 91 & 14 & 18.8725 & 6.3800 & 6.1825 \\
\hline 10 Aug 91 & 15 & 18.9525 & 6.3750 & 6.1825 \\
\hline
\end{tabular}




\section{C-28}

Table C.1 (continued)

\begin{tabular}{|c|c|c|c|c|}
\hline Date & Hour & $\begin{array}{c}\text { Temperature } \\
\left({ }^{\circ} \mathrm{C}\right)\end{array}$ & pH & $\begin{array}{c}\text { Dissolved } \\
\text { oxygen } \\
(\mathrm{mg} / \mathrm{L})\end{array}$ \\
\hline 10 Aug 91 & 16 & 18.9600 & 6.3450 & 6.1625 \\
\hline 10 Aug 91 & 17 & 19.0750 & 6.3150 & 6.1325 \\
\hline 10 Aug 91 & 18 & 19.3100 & 6.3950 & 6.1100 \\
\hline 10 Aug 91 & 19 & 19.5750 & 6.3650 & 6.0625 \\
\hline 10 Aug 91 & 20 & 18.9275 & 6.3875 & 6.0450 \\
\hline 10 Aug 91 & $? 1$ & 18.7120 & 6.4260 & 6.0460 \\
\hline 10 Aug 91 & 22 & 18.7475 & 6.4275 & 6.0050 \\
\hline 10 Aug 91 & 23 & 18.7525 & 6.3625 & 5.9725 \\
\hline 11 Aug 91 & 0 & & & \\
\hline 12 Aug 91 & 0 & 18.9500 & 6.2750 & 5.8400 \\
\hline 12 Aug 91 & 1 & 18.9350 & 6.2800 & 5.8300 \\
\hline 12 Aug 91 & 2 & 18.9250 & 6.2700 & 5.2050 \\
\hline 12 Aug 91 & 3 & 18.9250 & 6.2700 & 5.8200 \\
\hline 12 Aug 91 & 4 & 18.9200 & 6.2800 & 5.7650 \\
\hline 12 Aug 91 & 5 & 18.9200 & 6.2950 & 5.8100 \\
\hline 12 Aug 91 & 6 & 18.9100 & 6.2900 & 5.7600 \\
\hline 12 Aug 91 & 7 & 18.9200 & 6.2550 & 5.7300 \\
\hline 12 Aug 91 & 8 & 18.9200 & 6.2300 & 5.7000 \\
\hline 12 Aug 91 & 9 & 18.9400 & 6.1700 & 5.7350 \\
\hline 12 Aug 91 & 10 & 18.9450 & 6.1700 & 5.8000 \\
\hline 12 Aug 91 & 11 & 19.1000 & 6.2100 & 5.8450 \\
\hline 12 Aug 91 & 12 & 19.2050 & 6.2150 & 5.8150 \\
\hline 12 Aug 91 & 13 & 19.1200 & 6.2100 & 5.8800 \\
\hline 12 Aug 91 & 14 & 18.9400 & 6.2450 & 5.8700 \\
\hline 12 Aug 91 & 15 & 19.2600 & 6.3050 & 5.9550 \\
\hline 12 Aug 91 & 16 & 19.7250 & 6.3850 & 6.0450 \\
\hline 12 Aug 91 & 17 & 19.8950 & 6.3950 & 6.0650 \\
\hline 12 Aug 91 & 18 & 20.0500 & 6.4250 & 6.0200 \\
\hline 12. Aug 91 & 19 & 20.8700 & 6.4050 & 5.8650 \\
\hline 12 Aug 91 & 20 & 20.9200 & 6.3850 & 5.8750 \\
\hline 12 Aug 91 & 21 & 21.3350 & 6.3550 & 5.6800 \\
\hline 12 Aug 91 & 22 & 19.6300 & 6.3050 & 5.9050 \\
\hline
\end{tabular}


Table C.1 (continued)

\begin{tabular}{|c|c|c|c|c|}
\hline Date & Hour & $\begin{array}{c}\text { Temperature } \\
\left({ }^{\circ} \mathrm{C}\right)\end{array}$ & $\mathrm{pH}$ & $\begin{array}{c}\text { Dissolved } \\
\text { axygen } \\
(\mathrm{mg} / \mathrm{L})\end{array}$ \\
\hline 12 Aug 91 & 23 & 19.4400 & 6.3000 & 5.9300 \\
\hline 13 Aug 91 & 0 & 19.4250 & 6.3050 & 5.9050 \\
\hline 13 Aug 91 & 1 & 19.4900 & 6.3200 & 5.8750 \\
\hline 13 Aug 91 & 2 & 19.4550 & 6.3250 & 5.8400 \\
\hline 13 Aug 91 & 3 & 19.4050 & 6.3300 & 5.8500 \\
\hline 13 Aug 91 & 4 & 19.4350 & 6.3350 & 5.8100 \\
\hline 13 Aug 91 & 5 & 19.4550 & 6.3200 & 5.8050 \\
\hline 13 Aug 91 & 6 & 19.4350 & 6.3250 & 5.7550 \\
\hline 13 Aug 91 & 7 & 19.4150 & 6.3250 & 5.7500 \\
\hline 13 Aug 91 & 8 & 19.4250 & 6.3200 & 5.7850 \\
\hline 13 Aug 91 & 9 & 19.4450 & 6.2850 & 5.8350 \\
\hline 13 Aug 91 & 10 & 19.4500 & 6.2650 & 5.8450 \\
\hline 13 Aug 91 & 11 & 19.4750 & 6.2550 & 5.8500 \\
\hline 13 Aug 91 & 12 & 19.6750 & 6.2900 & 5.9050 \\
\hline 13 Aug 91 & 13 & 19.7133 & 6.2867 & 5.9500 \\
\hline 13 Aug 91 & 14 & 19.3600 & 6.3000 & 5.9700 \\
\hline 14 Aug 91 & 0 & & & \\
\hline 29 Aug 91 & 16 & 19.8000 & 6.4100 & 5.1300 \\
\hline 29 Aug 91 & 17 & 19.7600 & 6.5200 & 5.1800 \\
\hline 29 Aug 91 & 18 & 19.5200 & 6.4800 & 5.1800 \\
\hline 29 Aug 91 & 19 & 19.4800 & 6.5100 & 5.1500 \\
\hline 29 Aug 91 & 20 & 19.3900 & 6.5600 & 5.1400 \\
\hline 29 Aug 91 & 21 & 19.3400 & 6.6200 & 5.1500 \\
\hline 29 Aug 91 & 22 & 19.5000 & 6.6300 & 5.1900 \\
\hline 29 Aug 91 & 23 & 19.7500 & 6.6500 & 5.1900 \\
\hline 30 Aug 91 & 0 & 19.7100 & 6.6200 & 5.1400 \\
\hline 30 Aug 91 & 1 & 19.3900 & 6.6000 & 5.1100 \\
\hline 30 Aug 91 & 2 & 19.2300 & 6.5900 & 5.1000 \\
\hline 30 Aug 91 & 3 & 19.2300 & 6.5800 & 5.0700 \\
\hline 30 Aug 91 & 4 & 19.2100 & 6.5800 & 5.0400 \\
\hline 30 Aug 91 & 5 & 19.2400 & 6.5800 & 5.0400 \\
\hline 30 Aug 91 & 6 & 19.2400 & 6.5800 & 5.0600 \\
\hline
\end{tabular}


Table C.1 (continued)

\begin{tabular}{|c|c|c|c|c|}
\hline Date & Hour & $\begin{array}{c}\text { Temperature } \\
\left({ }^{\circ} \mathrm{C}\right)\end{array}$ & $\mathrm{pH}$ & $\begin{array}{c}\text { Dissolved } \\
\text { axygen } \\
\text { (mg/L) }\end{array}$ \\
\hline 30 Aug 91 & 7 & 19.2400 & 6.5800 & 51200 \\
\hline 30 Aug 91 & 8 & 19.2300 & 6.5700 & 5.1200 \\
\hline 30 Aug 91 & 9 & 19.2400 & 6.5800 & 5.1400 \\
\hline 30 Aug 91 & 10 & 19.5500 & 6.6000 & 5.1700 \\
\hline 30 Aug 91 & 11 & $1 y .5300$ & 6.6000 & 5.1700 \\
\hline 30 Aug 91 & 12 & 19.5400 & 6.6200 & 5.2800 \\
\hline 30 Aug 91 & 13 & 19.7300 & 6.6600 & 5.3100 \\
\hline 30 Aug 91 & 14 & 19.9500 & 6.6800 & 5.3500 \\
\hline 30 Aug 91 & 15 & 19.9100 & 6.6800 & 5.38001 \\
\hline 30 Aug 91 & 16 & 20.0500 & 6.7100 & 5.4600 \\
\hline 30 Aug 91 & 17 & 19.9600 & 6.7300 & 5.4800 \\
\hline 30 Aug 91 & 18 & 20.0900 & 6.7600 & 5.5100 \\
\hline 30 Aug 91 & 19 & 20.1100 & 6.7400 & 5.5000 \\
\hline 30 Aug 91 & 20 & 19.8600 & 6.7100 & 5.4200 \\
\hline 30 Aug 91 & 21 & 19.8500 & 6.7100 & 5.4200 \\
\hline 30 Aug 91 & 22 & 19.9700 & 6.7300 & 5.4900 \\
\hline 30 Aug 91 & 23 & 19.9600 & 6.7100 & 5.4400 \\
\hline 31 Aug 91 & 0 & 19.9000 & 6.6300 & 5.3900 \\
\hline 31 Aug 91 & 1 & 19.8400 & 6.6200 & 5.3800 \\
\hline 31 Aug 91 & 2 & 19.7800 & 6.6000 & 5.3500 \\
\hline 31 Aug 91 & 3 & 19.8300 & 6.6000 & 5.3600 \\
\hline 31 Aug 91 & 4 & 19.7700 & 6.6500 & 5.3000 \\
\hline 31 Aug 91 & 5 & 19.7600 & 6.6400 & 5.2800 \\
\hline 31 Aug 91 & 6 & 19.7600 & 6.6500 & 5.2800 \\
\hline 31 Aug 91 & 7 & 19.7600 & 6.6700 & 5.3100 \\
\hline 31 Aug 91 & 8 & 19.7700 & 6.6600 & 5.3000 \\
\hline 31 Aug 91 & 9 & 19.8000 & 6.6700 & 5.3200 \\
\hline 31 Aug 91 & 10 & 19.8100 & 6.6500 & 5.3400 \\
\hline 31 Aug 91 & 11 & 19.8100 & 6.6800 & 5.3400 \\
\hline 31 Aug 91 & 12 & 20.1600 & 6.6900 & 5.3700 \\
\hline 31 Aug 91 & 13 & 20.2400 & 6.7100 & 5.3500 \\
\hline 31 Aug 91 & 14 & 20.0100 & 6.6400 & 5.3400 \\
\hline
\end{tabular}




\section{C-31}

Table C.1 (continued)

\begin{tabular}{|c|c|c|c|c|}
\hline Date & Hour & $\begin{array}{c}\text { Temperature } \\
\left({ }^{\circ} \mathrm{C}\right)\end{array}$ & $\mathrm{pH}$ & $\begin{array}{c}\text { Dissolved } \\
\text { axygen } \\
(\mathrm{mg} / \mathrm{L})\end{array}$ \\
\hline 31 Aug 91 & 15 & 19.8600 & 6.5900 & 5.3600 \\
\hline 31 Aug 91 & 16 & 19.8600 & 6.4900 & 5.3500 \\
\hline 31 Aug 91 & 17 & 19.7800 & 6.6600 & 5.3300 \\
\hline 31 Aug 91 & 18 & 19.7400 & 6.6100 & 5.3700 \\
\hline 31 Aug 91 & 19 & 19.8500 & 6.6800 & 5.4500 \\
\hline 31 Aug 91 & 20 & 20.0700 & 6.7300 & 5.4700 \\
\hline 31 Aug 91 & 21 & 20.2700 & 6.7000 & 5.5500 \\
\hline 31 Aug 91 & $2 ! !$ & 20.1700 & 6.6800 & 5.4900 \\
\hline 31 Aug 91 & 23 & 19.7000 & 6.6300 & 5.4200 \\
\hline 01 Sep 91 & 0 & 19.7300 & 6.6200 & 5.4000 \\
\hline 01 Sep 91 & 1 & 19.6100 & 6.5800 & 5.4000 \\
\hline 01 Sep 91 & 2 & 19.6300 & 6.6500 & 5.4100 \\
\hline 01 Sep 91 & 3 & 19.5700 & 6.6100 & 5.3300 \\
\hline 01 Sep 91 & 4 & 19.5500 & 6.6100 & 5.3400 \\
\hline 01 Sep 91 & 5 & 19.5400 & 6.5300 & 5.3500 \\
\hline 01 Sep 91 & 6 & 19.5400 & 6.5900 & 5.3500 \\
\hline 01 Sep 91 & 7 & 19.5100 & 6.5400 & 5.3300 \\
\hline 01 Sep 91 & 8 & 19.5200 & 6.5800 & 5.3200 \\
\hline 01 Sep 91 & 9 & 19.5300 & 6.6200 & 5.3100 \\
\hline 01 Sep 91 & 10 & 19.5300 & 6.6000 & 5.3200 \\
\hline 01 Sep 91 & 11 & 19.5400 & 6.5700 & 5.3800 \\
\hline 01 Sep 91 & 12 & 19.9800 & 6.6300 & 5.4400 \\
\hline $01 \operatorname{Sep} 91$ & 13 & 20.0900 & 6.5600 & 5.4300 \\
\hline $01 \operatorname{Sep} 91$ & 14 & 19.9300 & 6.4100 & 5.4200 \\
\hline 01 Sep 91 & 15 & 19.8900 & 6.4500 & 5.4600 \\
\hline 01 Sep 91 & 16 & 19.9000 & 6.4700 & 5.4500 \\
\hline 01 Sep 91 & 17 & 19.7900 & 6.5000 & 5.4200 \\
\hline 01 Sep 91 & 18 & 19.6800 & 6.5000 & 5.3900 \\
\hline 01 Sep 91 & 19 & 19.5900 & 6.4800 & 5.3600 \\
\hline 01 Sep 91 & 20 & 19.5700 & 6.5500 & 5.3600 \\
\hline 01 Sep 91 & 21 & 19.5100 & 6.4100 & 5.3400 \\
\hline 01 Sep 91 & 22 & 19.4900 & 6.4400 & 5.3300 \\
\hline
\end{tabular}


Table C.1 (continued)

\begin{tabular}{|c|c|c|c|c|}
\hline Date & Hour & $\begin{array}{c}\text { Temperature } \\
\left({ }^{\circ} \mathrm{C}\right)\end{array}$ & pH & $\begin{array}{c}\text { Dissolved } \\
\text { oxygen } \\
(\mathrm{mg} / \mathrm{L})\end{array}$ \\
\hline 01 Sep 91 & 23 & 19.3900 & 6.5300 & 5.3000 \\
\hline $02 \operatorname{Sep} 91$ & 0 & 19.3800 & 6.4500 & 5.2900 \\
\hline 02 Sep 91 & 1 & 19.3700 & 6.5800 & 5.2700 \\
\hline $02 \operatorname{Sep} 91$ & 2 & 19.3700 & 6.5000 & 5.2700 \\
\hline $02 \operatorname{Sep} 91$ & 3 & 19.3600 & 6.5300 & 5.2500 \\
\hline 02 Sep 91 & 4 & 19.3500 & 6.5800 & 5.2500 \\
\hline $02 \operatorname{Sep} 91$ & 5 & 19.3300 & 6.5000 & 5.2100 \\
\hline $02 \operatorname{Sep} 91$ & 6 & 19.3300 & 6.5000 & 5.2200 \\
\hline 02 Sep 91 & 7 & 19.3400 & 6.5200 & 5.2200 \\
\hline $02 \operatorname{Sep} 91$ & 8 & 19.3400 & 6.5000 & $5.220 \mathrm{C}$ \\
\hline 02 Sep 91 & 9 & 19.3600 & 6.5200 & 5.2400 \\
\hline $02 \operatorname{Sep} 91$ & -10 & 19.3600 & 6.4700 & 5.2600 \\
\hline O2 Sep 91 & 11 & 19.3700 & 6.4000 & 5.2800 \\
\hline 02 Sep 91 & 12 & 19.3800 & 6.4000 & 5.2600 \\
\hline $02 \operatorname{Sep} 91$ & 13 & 19.9400 & 6.5100 & 5.3200 \\
\hline 02 Sep 91 & 14 & 19.8700 & 6.4900 & 5.3000 \\
\hline 02 Sep 91 & 15 & 19.7900 & 6.5000 & 5.3300 \\
\hline 02 Sep 91 & 16 & 19.7200 & 6.4900 & 5.2900 \\
\hline $02 \operatorname{Sep} 91$ & 17 & 19.6800 & 6.4600 & 5.2700 \\
\hline $02 \operatorname{Sep} 91$ & 18 & 19.5700 & 6.4900 & 5.2500 \\
\hline $02 \operatorname{Sep} 91$ & 19 & 19.4200 & 6.4600 & 5.2200 \\
\hline 02 Sep 91 & 20 & 19.4000 & 6.4300 & 5.2200 \\
\hline 02 Sep 91 & 21 & 13.3200 & 6.4900 & 5.2100 \\
\hline 02 Sep 91 & 22 & 19.3400 & 6.4700 & 5.2300 \\
\hline 02 Sep 91 & 23 & 19.1700 & 6.4500 & 5.2100 \\
\hline $03 \operatorname{Sep} 91$ & 0 & 19.0ن00 & 6.4500 & 5.2000 \\
\hline 03 Sep 91 & 1 & 19.0800 & 6.4300 & 5.2000 \\
\hline 03 Sep 91 & 2 & 19.0900 & 6.4400 & 5.1800 \\
\hline 03 Sep \$1 & 3 & 19.0900 & 6.4600 & 5.1900 \\
\hline 03 Sep 91 & 4 & 19.0800 & 6.4800 & 5.1600 \\
\hline 03 Sep 91 & 5 & 19.0800 & 6.4300 & 5.1600 \\
\hline 03 Sep 91 & 6 & 19.0800 & 6.4600 & 5.1500 \\
\hline
\end{tabular}




\section{C-33}

Table C.1 (continued)

\begin{tabular}{|c|c|c|c|c|}
\hline Date & Hour & $\begin{array}{l}\text { Temperature } \\
\left({ }^{\circ} \mathrm{C}\right)\end{array}$ & $\mathrm{pH}$ & $\begin{array}{c}\text { Dissolved } \\
\text { axygen } \\
(\mathrm{mg} / \mathrm{L})\end{array}$ \\
\hline 03 Sep 91 & 7 & 19.0800 & 6.5000 & 5.1200 \\
\hline $03 \mathrm{Se}$, 91 & 8 & 19.0800 & 6.4800 & 5.1200 \\
\hline 03 Sep 91 & 9 & 19.0800 & 6.4400 & 5.1200 \\
\hline 03 Sep 91 & 10 & 19.1000 & 6.3800 & 5.1600 \\
\hline 03 Sep 91 & 11 & 19.3700 & 6.4400 & 5.1900 \\
\hline 03 Sep 91 & 12 & 19.4600 & 6.4700 & 5.1700 \\
\hline 03 Sep 91 & 13 & 19.4200 & 6.4500 & 5.1600 \\
\hline 03 Sep 91 & 14 & 19.4000 & 6.4400 & 5.1300 \\
\hline 03 Sep 91 & 15 & 19.5700 & 6.5100 & 5.2100 \\
\hline 03 Sep 91 & 16 & 19.7000 & 6.5800 & 5.2600 \\
\hline 03 Sep 91 & 17 & 19.7400 & 6.5100 & 5.2800 \\
\hline 03 Sep 91 & 18 & 19.7400 & 6.5500 & 5.2700 \\
\hline 03 Sep 91 & 19 & 19.8400 & 6.5600 & 5.3200 \\
\hline 03 Sep 91 & 20 & 19.8900 & 6.5500 & 5.2800 \\
\hline 03 Sep 91 & 21 & 19.8300 & 6.5800 & 5.2700 \\
\hline 03 Sep 91 & 22 & 19.6900 & 6.5300 & 5.2600 \\
\hline $03 \operatorname{Sep} 91$ & 23 & 19.5900 & 6.5400 & 5.2900 \\
\hline 04 Sep 91 & 0 & 19.5200 & 6.5700 & 5.2600 \\
\hline 04 Sep 91 & 1 & 19.5400 & 6.5000 & 5.2700 \\
\hline 04 Sep 91 & 2 & 19.4200 & 6.5200 & 5.2100 \\
\hline 04 Sep 91 & 3 & 19.4400 & 6.5300 & 5.2400 \\
\hline 04 Sep 91 & 4 & 19.4600 & 6.4500 & 5.2400 \\
\hline 04 Sep 91 & 5 & 19.4400 & 6.4500 & 5.2100 \\
\hline 04 Sep 91 & 6 & 19.4400 & 6.4500 & 5.2000 \\
\hline 04 Sep 91 & 7 & 19.4000 & 6.4300 & 5.1800 \\
\hline 04 Sep 91 & 8 & 19.4400 & 6.5300 & 5.1900 \\
\hline 04 Sep 91 & 9 & 19.4200 & 6.5500 & 5.1600 \\
\hline 04 Sep 91 & 10 & 19.4100 & 6.4600 & 5.1400 \\
\hline 04 Sep 91 & 11 & 19.5600 & 6.4100 & 5.1800 \\
\hline 04 Sep 91 & 12 & 19.9000 & 6.4700 & 5.2400 \\
\hline 04 Sep 91 & 13 & 19.7400 & 6.4000 & 5.1700 \\
\hline 04 Sep 91 & 14 & 19.9000 & 6.4800 & 5.2600 \\
\hline
\end{tabular}




\section{C-34}

Table C.1 (continued)

\begin{tabular}{|c|c|c|c|c|}
\hline Date & Hour & $\begin{array}{c}\text { Temperature } \\
\left({ }^{\circ} \mathrm{C}\right)\end{array}$ & $\mathrm{pH}$ & $\begin{array}{c}\text { Dissolved } \\
\text { axygen } \\
(\mathrm{mg} / \mathrm{L})\end{array}$ \\
\hline 04 Sep 91 & 15 & 20.1300 & 6.5200 & 5.3100 \\
\hline $04 \operatorname{Sep} 91$ & 16 & 20.0500 & 6.4700 & 5.3100 \\
\hline 04 Sep 91 & 17 & 19.9500 & 6.4500 & 5.2700 \\
\hline 04 Sep 91 & 18 & 20.0000 & 6.5000 & 5.2200 \\
\hline 04 Sep 91 & 19 & 19.9100 & 6.4700 & 5.2300 \\
\hline 04 Sep 91 & 20 & 19.8000 & 6.4600 & 5.2500 \\
\hline 04 Sep 91 & 21 & 19.8400 & 6.5000 & 5.2400 \\
\hline $04 \operatorname{Sep} 91$ & 22 & 19.6800 & 6.5300 & 5.2500 \\
\hline 04 Sep 91 & 23 & 19.7100 & 6.4900 & 5.2400 \\
\hline 05 Sep 91 & 0 & 19.5900 & 6.5200 & 5.2200 \\
\hline 05 Sep 91 & 1 & 19.5700 & 6.5200 & 5.2200 \\
\hline 05 Sep 91 & 2 & 19.5800 & 6.4900 & 5.2100 \\
\hline 05 Sep 91 & 3 & $19.57 C 0$ & 6.4500 & 5.2100 \\
\hline 05 Sep 91 & 4 & 19.5500 & 6.4900 & 5.2000 \\
\hline 05 Sep 91 & 5 & 19.5500 & 6.4900 & 5.1900 \\
\hline 05 Sep 91 & 6 & 19.5500 & 6.4500 & 5.1800 \\
\hline 05 Sep 91 & 7 & 19.5500 & 6.4600 & 5.1600 \\
\hline 05 Sep 91 & 8 & 19.5400 & 6.4100 & 5.1700 \\
\hline 05 Sep 91 & 9 & 19.5200 & 6.4600 & 5.1200 \\
\hline 05 Sep 91 & 10 & 19.5400 & 6.4300 & 5.1900 \\
\hline 05 Sep 91 & 11 & 19.5900 & 6.4500 & 5.2000 \\
\hline 05 Sep 91 & 12 & 19.5300 & 6.4100 & 5.2100 \\
\hline $05 \operatorname{Sep} 91$ & 13 & 19.6000 & 6.4100 & 5.2600 \\
\hline 05 Sep 91 & 14 & 19.6300 & 6.4400 & 5.3300 \\
\hline 05 Sep 91 & 15 & 19.9200 & 6.4800 & 5.3600 \\
\hline $05 \operatorname{Sep} 91$ & 16 & 20.2100 & 6.5500 & 5.2700 \\
\hline $05 \operatorname{Sep} 91$ & 17 & 19.7800 & 6.4900 & 5.2300 \\
\hline 05 Sep 91 & 18 & 19.4300 & 6.5000 & 5.0800 \\
\hline $05 \operatorname{Sep} 91$ & 19 & 19.4500 & 6.5500 & 5.0400 \\
\hline 05 Sep 91 & 20 & 19.5100 & 6.5600 & 5.0300 \\
\hline 05 Sep 91 & 21 & 19.3900 & 6.5600 & 5.0300 \\
\hline $05 \operatorname{Sep} 91$ & 22 & 19.2100 & 6.5500 & 5.0400 \\
\hline
\end{tabular}




\section{C-35}

Table C.1 (continued)

\begin{tabular}{|c|c|c|c|c|}
\hline Date & Hour & $\begin{array}{c}\text { Temperature } \\
\left({ }^{\circ} \mathrm{C}\right)\end{array}$ & pH & $\begin{array}{c}\text { Dissotved } \\
\text { rejgen } \\
\text { (mg/L) }\end{array}$ \\
\hline 05 Sep 91 & 23 & 19.1300 & 6.5400 & 5.0500 \\
\hline O6 Sep 91 & 0 & 19.1600 & 6.5300 & 5.0300 \\
\hline 06 Sep 91 & 1 & 19.1900 & 6.5500 & 5.0300 \\
\hline CS Sep 91 & 2 & 19.1600 & 6.5500 & 5.0300 \\
\hline 06 Sep 91 & 3 & 19.1500 & 6.5500 & 5.0600 \\
\hline 06 Sep 91 & 4 & 19.1400 & 6.5500 & 5.0500 \\
\hline $06 \operatorname{Sep} 91$ & 5 & 19.1200 & 6.5400 & 5.0300 \\
\hline 06 Sep 91 & 6 & 19.1200 & 6.5400 & 5.0300 \\
\hline 06 Sep 91 & 7 & 19.1200 & 6.5400 & 5.0200 \\
\hline 06 Sep 91 & 8 & 19.1400 & 6.5400 & 5.0200 \\
\hline 06 Sep 91 & 9 & 19.1200 & 6.5300 & 4.9800 \\
\hline 06 Sep 91 & 10 & 19.1500 & 6.5500 & 5.0400 \\
\hline 06 Sep 91 & 11 & 19.1800 & 6.5600 & 5.0800 \\
\hline 06 Sep 91 & 12 & 19.4200 & 6.5000 & 5.0600 \\
\hline $06 \operatorname{Sep} 91$ & 13 & 19.5600 & 6.4900 & 5.0200 \\
\hline 06 Sep 91 & 14 & 19.5600 & 6.4900 & 5.0600 \\
\hline 06 Sep 91 & 15 & 20.1000 & 6.5200 & 4.9900 \\
\hline 06 Sep 91 & 16 & 19.8600 & 6.5000 & 5.0400 \\
\hline 06 Sep 91 & 17 & 19.7800 & 6.4800 & 5.0300 \\
\hline 06 Sep 91 & 18 & 19.6700 & 6.5100 & 5.0000 \\
\hline 06 Sep 91 . & 19 & 19.6000 & 6.4000 & 5.0000 \\
\hline 06 Sep 91 & 20 & 19.4900 & 6.4700 & 5.0300 \\
\hline 06 Sep 91 & 21 & 19.6400 & 6.4600 & 5.0400 \\
\hline 06 Sep 91 & 22 & 19.5700 & 6.4800 & 5.1100 \\
\hline 06 Sep 91 & 23 & 19.4500 & 6.5100 & 5.1100 \\
\hline 07 Sep 91 & 0 & 19.4100 & 6.4400 & 5.1300 \\
\hline $07 \operatorname{Sep} 91$ & 1 & 19.3100 & 6.4400 & 5.1300 \\
\hline 07 Sep 91 & 2 & 19.2800 & 6.4500 & 5.1200 \\
\hline 07 Sep 91 & 3 & 19.3000 & 6.5500 & 5.0900 \\
\hline 07 Sep 91 & 4 & 19.2700 & 6.5400 & 5.0900 \\
\hline 07 Sep 91 & 5 & 19.2400 & 6.5500 & 5.0800 \\
\hline 07 Sep 91 & 6 & 19.2700 & 6.5500 & 5.0500 \\
\hline
\end{tabular}


Table C.1 (continued)

\begin{tabular}{|c|c|c|c|c|}
\hline Date & Hour & $\begin{array}{c}\text { Temperature } \\
\left({ }^{\circ} \mathrm{C}\right)\end{array}$ & $\mathrm{pH}$ & $\begin{array}{c}\text { Dissolved } \\
\text { oxygen } \\
(\mathrm{mg} / \mathrm{L})\end{array}$ \\
\hline 07 Sep 91 & 7 & 19.3500 & 6.5500 & 5.0200 \\
\hline 07 Sep 91 & 8 & 19.2600 & 6.5600 & 5.0500 \\
\hline 07 Sep 91 & 9 & 19.2300 & 6.5600 & 5.0600 \\
\hline 07 Sep 91 & 10 & 19.2600 & 6.5400 & 5.0200 \\
\hline 07 Sep 91 & 11 & 19.5200 & 6.5200 & 5.0600 \\
\hline 07 Sep 91 & 12 & 19.7500 & 6.4900 & 5.0800 \\
\hline 07 Sep 91 & 13 & 19.8300 & 6.5400 & 5.1200 \\
\hline 07 Sep 91 & 14 & 20.1900 & 6.6200 & 5.1700 \\
\hline 07 Sep 91 & 15 & 20.3200 & 6.6400 & 5.2400 \\
\hline 07 Sep 91 & 16 & 20.4500 & 6.6200 & 5.2700 \\
\hline 07 Sep 91 & 17 & 20.4400 & 6.6500 & 5.3100 \\
\hline 07 Sep 91 & 18 & 20.3400 & 6.6400 & 5.3100 \\
\hline 07 Sep 91 & 19 & 20.3800 & 6.6200 & 5.3300 \\
\hline 07 Sep 91 & 20 & 20.4400 & 6.6300 & 5.3500 \\
\hline 07 Sep 91 & 21 & 20.4300 & 6.6400 & 5.3700 \\
\hline 07 Sep 91 & 22 & 20.5300 & 6.6300 & 5.3100 \\
\hline 07 Sep 91 & 23 & 20.4800 & 6.6100 & 5.2900 \\
\hline 08 Sep 91 & 0 & 20.3900 & 6.5700 & 5.2800 \\
\hline 08 Sep 91 & 1 & 20.3500 & 6.6000 & 5.3200 \\
\hline 08 Sep 91 & 2 & 20.1800 & 6.5700 & 5.3200 \\
\hline 08 Sep 91 & 3 & 20.1900 & 6.5900 & 5.3100 \\
\hline 08 Sep 91 & 4 & 20.1600 & 6.5600 & 5.2800 \\
\hline 08 Sep 91 & 5 & 20.1600 & 6.5300 & 5.2800 \\
\hline 08 Sep 91 & 6 & 20.2400 & 6.5600 & 5.3000 \\
\hline 08 Sep 91 & 7 & 20.2800 & 6.5600 & 5.2700 \\
\hline 08 Sep 91 & 8 & 20.1900 & 6.5700 & 5.2600 \\
\hline 08 Sep 91 & 9 & 20.1100 & 6.5800 & 5.2900 \\
\hline 08 Sep 91 & 10 & 20.2200 & 6.6000 & 5.3000 \\
\hline 08 Sep 91 & 11 & 20.3200 & 6.5800 & 5.1900 \\
\hline 08 Sep 91 & 12 & 20.3800 & 6.6000 & 5.3000 \\
\hline 08 Sep 91 & 13 & 20.6300 & 6.5900 & 5.3100 \\
\hline 08 Sep 91 & 14 & 20.5300 & 6.6100 & 5.3100 \\
\hline
\end{tabular}




\section{C-37}

Table C.1 (continued)

\begin{tabular}{|c|c|c|c|c|}
\hline Date & Hour & $\begin{array}{c}\text { Temperature } \\
\left({ }^{\circ} \mathrm{C}\right)\end{array}$ & $\mathrm{pH}$ & $\begin{array}{c}\text { Dissolved } \\
\text { axygen } \\
\text { (mg/L) }\end{array}$ \\
\hline 08 Sep 91 & 15 & 20.4600 & 6.6000 & 5.3600 \\
\hline 08 Sep 91 & 16 & 20.5900 & 6.5700 & 5.3400 \\
\hline 08 Sep 91 & 17 & 20.4300 & 6.5800 & 5.3400 \\
\hline 08 Sep 91 & 18 & 20.1800 & 6.6000 & 5.2700 \\
\hline 08 Sep 91 & 19 & 20.1600 & 6.6200 & 5.2700 \\
\hline 08 Sep 91 & 20 & 20.5200 & 6.5900 & 5.2400 \\
\hline 08 Sep 91 & 21 & 20.4700 & 6.6200 & 5.3100 \\
\hline 08 Sep 91 & 22 & 20.2500 & 6.5900 & 5.3300 \\
\hline 08 Sep 91 & 23 & 20.1000 & 6.6200 & 5.3600 \\
\hline 09 Sep 91 & 0 & 20.0700 & 6.6100 & 5.3500 \\
\hline 09 Sep 91 & 1 & 20.0600 & 6.6000 & 5.3300 \\
\hline 09 Sep 91 & 2 & 20.1200 & 6.6100 & 5.3300 \\
\hline 09 Sep 91 & 3 & 20.1000 & 6.5400 & 5.3300 \\
\hline 09 Sep 91 & 4 & 20.0500 & 6.5600 & 5.3100 \\
\hline 09 Sep 91 & 5 & 20.0500 & 6.5500 & 5.2900 \\
\hline 09 Sep 91 & 6 & 20.0500 & 6.5300 & 5.3000 \\
\hline 09 Sep 91 & 7 & 20.0400 & 6.4900 & 5.2800 \\
\hline $09 \operatorname{Sep} 91$ & 8 & 20.0200 & 6.5400 & 5.2700 \\
\hline 09 Sep 91 & 9 & 20.0200 & 6.6200 & 5.3000 \\
\hline $09 \operatorname{Sep} 91$ & 10 & 20.1200 & 6.5900 & 5.3200 \\
\hline 09 Sep 91 & 11 & 20.2800 & 6.5100 & 5.3200 \\
\hline C9 Sep 91 & 12 & 20.3000 & 6.5200 & 5.3100 \\
\hline 09 Sep 91 & 13 & 20.1300 & 6.5000 & 5.3200 \\
\hline 09 Sep 91 & 14 & 20.1300 & 6.1500 & 5.6400 \\
\hline 09 Sep 91 & 15 & 19.8100 & 5.5800 & 6.2800 \\
\hline 09 Sep 91 & 16 & 19.8300 & 5.6400 & 6.2100 \\
\hline 09 Sep 91 & 17 & 19.8100 & 5.6700 & 6.2000 \\
\hline 09 Sep 91 & 18 & 19.7500 & 5.6800 & 6.1400 \\
\hline 09 Sep 91 & 19 & 19.7200 & 5.6700 & 6.0800 \\
\hline 09 Sep 91 & 20 & 19.6200 & 5.7100 & 6.2000 \\
\hline 09 Sep 91 & 21 & 19.7300 & 5.7300 & 6.2400 \\
\hline 09 Sep 91 & 22 & 19.9800 & 5.7700 & 6.3300 \\
\hline
\end{tabular}




\section{C-38}

Table C.1 (continued)

\begin{tabular}{|c|c|c|c|c|}
\hline Date & Hour & $\begin{array}{c}\text { Temperature } \\
\left({ }^{\circ} \mathrm{C}\right)\end{array}$ & pH & $\begin{array}{l}\text { Dissolved } \\
\text { axygen } \\
(\mathrm{mg} / \mathrm{L})\end{array}$ \\
\hline 09 Sep 91 & 23 & 19.9400 & 5.7600 & 6.3800 \\
\hline 10 Sep 91 & 0 & 19.6700 & 5.7400 & 6.3100 \\
\hline 10 Sep 91 & 1 & 19.6300 & 5.7400 & 6.3200 \\
\hline 10 Sep 91 & 2 & 19.6400 & 5.7500 & 6.3300 \\
\hline 10 Sep 91 & 3 & 19.6400 & 5.7600 & 6.3200 \\
\hline $10 \operatorname{Sep} 91$ & 4 & 19.6300 & 5.7500 & 6.3000 \\
\hline $10 \operatorname{Sep} 91$ & 5 & 19.6300 & 5.7700 & 6.2900 \\
\hline 10 Sep 91 & 6 & 19.6200 & 5.7700 & 6.2700 \\
\hline 10 Sep 91 & 7 & 19.6200 & 5.7800 & 6.2900 \\
\hline 10 Sep 91 & 8 & 19.6100 & 5.7700 & 6.2800 \\
\hline $10 \operatorname{Sep} 91$ & 9 & 19.6100 & 5.7800 & 6.2800 \\
\hline 10 Sep 91 & 10 & 19.6300 & 5.7800 & 6.3300 \\
\hline $10 \operatorname{Sep} 91$ & 11 & 19.6200 & 5.7800 & 6.3200 \\
\hline 10 Sep 91 & 12 & 19.6200 & 5.7900 & 7.5600 \\
\hline 11 Sep 91 & 0 & & & \\
\hline 17 Sep 91 & 12 & 20.7600 & 8.2300 & 8.2300 \\
\hline 17 Sep 91 & 13 & 20.5700 & 8.2200 & 7.6200 \\
\hline 17 Sep 91 & 14 & 20.3900 & 8.2000 & 7.6500 \\
\hline $17 \operatorname{Sep} 91$ & 15 & 20.3800 & 8.2000 & 7.9100 \\
\hline 17 Sep 91 & 16 & 20.4900 & 8.2200 & 10.6000 \\
\hline 17 Sep 91 & 17 & 20.7000 & 8.2600 & 10.9400 \\
\hline 17 Sep 91 & 18 & 20.5300 & 8.2500 & 10.9900 \\
\hline 17 Sep 91 & 19 & 20.3800 & 8.2300 & 11.0100 \\
\hline 17 Sep 91 & 20 & 20.3900 & 8.2300 & 11.0100 \\
\hline 17 Sep 91 & 21 & 20.5400 & 8.2400 & 10.8900 \\
\hline $17 \operatorname{Sep} 91$ & 22 & 20.6200 & 8.2500 & 10.9500 \\
\hline 17 Sep 91 & 23 & 20.7000 & 8.2400 & 10.8200 \\
\hline $18 \operatorname{Sep} 91$ & 0 & 20.5300 & 8.2600 & 10.9900 \\
\hline $18 \operatorname{Sep} 91$ & 1 & 20.4600 & 8.2500 & 11.0000 \\
\hline 18 Sep 91 & 2 & 20.4600 & 8.2400 & 11.0500 \\
\hline $18 \operatorname{Sep} 91$ & 3 & 20.4500 & 8.2400 & 11.0800 \\
\hline 18 Sep 91 & 4 & 20.4900 & 8.2500 & 11.0600 \\
\hline
\end{tabular}




\section{C-39}

Table C.1 (continued)

\begin{tabular}{|c|c|c|c|c|}
\hline Date & Hour & $\begin{array}{c}\text { Temperature } \\
\left({ }^{\circ} \mathrm{C}\right)\end{array}$ & $\mathrm{pH}$ & $\begin{array}{c}\text { Dissolved } \\
\text { orygen } \\
(\mathrm{mg} / \mathrm{L})\end{array}$ \\
\hline 18 Sep 91 & 5 & 20.4800 & 8.2500 & 10.9400 \\
\hline $18 \operatorname{Sep} 91$ & 6 & 20.4700 & 8.2300 & 10.8100 \\
\hline 18 Sep 91 & 7 & 20.4400 & 8.2200 & 10.8500 \\
\hline 18 Sep 91 & 8 & 20.4800 & 8.2200 & 10.7100 \\
\hline 18 Sep 91 & 9 & 20.5200 & 8.2400 & 10.8600 \\
\hline $18 \operatorname{Sep} 91$ & 10 & 20.5600 & 8.2500 & 10.7700 \\
\hline 18 Sep 91 & 11 & 20.6100 & 8.3100 & 11.0600 \\
\hline 18 Sep 91 & 12 & 20.7000 & 8.3500 & 11.3100 \\
\hline 18 Sep 91 & 13 & 20.9900 & 8.3200 & 11.0000 \\
\hline 18 Sep 91 & 14 & 20.7600 & 8.2900 & 10.8500 \\
\hline $18 \operatorname{Sep} 91$ & 15 & 20.4000 & 8.2200 & 10.3200 \\
\hline $18 \operatorname{Sep} 91$ & 16 & 20.3200 & 8.2000 & 10.6100 \\
\hline 18 Sep 91 & 17 & 20.1700 & 8.2000 & 10.4700 \\
\hline $18 \operatorname{Sep} 91$ & 18 & 20.2700 & 8.2200 & 10.5800 \\
\hline 18 Sep 91 & 19 & 20.2900 & 8.2100 & 10.5000 \\
\hline 18 Sep 91 & 20 & 20.2700 & 8.2100 & 11.0900 \\
\hline 18 Sep 91 & 21 & 20.2200 & 8.2100 & 10.9500 \\
\hline 18 Sep 91 & 22 & 20.0500 & 8.1800 & 10.2000 \\
\hline $18 \operatorname{Sep} 91$ & 23 & 20.2800 & 8.1900 & 10.2600 \\
\hline 19 Sep 91 & 0 & 20.2800 & 8.1900 & 10.0000 \\
\hline 19 Sep 91 & 1 & 20.1700 & 8.1800 & 9.7700 \\
\hline 19 Sep 91 & 2 & 20.1600 & 8.1800 & 9.9700 \\
\hline $19 \operatorname{Sep} 91$ & 3 & 20.1900 & 8.1800 & 9.9000 \\
\hline 19 Sep 91 & 4 & 20.2200 & 8.1700 & 9.6700 \\
\hline $19 \operatorname{Sep} 91$ & 5 & 20.3600 & 8.1800 & 9.8000 \\
\hline 19 Sep 91 & 6 & 20.3500 & 8.1700 & 9.7600 \\
\hline 19 Sep 91 & 7 & 20.2400 & 8.1700 & 9.6100 \\
\hline $19 \operatorname{Sep} 91$ & 8 & 20.2300 & 8.1800 & 9.7300 \\
\hline 19 Sep 91 & 9 & 20.3500 & 8.1700 & 9.6300 \\
\hline $19 \operatorname{Sep} 91$ & 10 & 20.1600 & 8.1600 & 9.6900 \\
\hline $19 \operatorname{Sep} 91$ & 11 & 20.0700 & 8.1500 & 9.7400 \\
\hline $19 \operatorname{Sep} 91$ & 12 & 20.0500 & 8.1700 & 9.6500 \\
\hline
\end{tabular}




\section{C- 40}

Table C.1 (continued)

\begin{tabular}{|c|c|c|c|c|}
\hline Date & Hour & $\begin{array}{c}\text { Temperature } \\
\left({ }^{\circ} \mathrm{C}\right)\end{array}$ & $\mathrm{pH}$ & $\begin{array}{c}\text { Dissolved } \\
\text { axygen } \\
(\mathrm{mg} / \mathrm{L})\end{array}$ \\
\hline 19 Sep 91 & 13 & 20.0700 & 8.1800 & 9.6900 \\
\hline $19 \operatorname{Sep} 91$ & 14 & 20.0900 & 8.1900 & 9.5800 \\
\hline 19 Sep 91 & 15 & 20.0900 & 8.1900 & 9.9300 \\
\hline $19 \operatorname{Sep} 91$ & 16 & 19.9000 & 8.1600 & 9.9100 \\
\hline 19 Sep 91 & 17 & 19.8300 & 8.1600 & 10.0300 \\
\hline 19 Sep 91 & 18 & 20.0200 & 8.1900 & 10.0000 \\
\hline 19 Sep 91 & 19 & 20.0900 & 8.2000 & 9.8700 \\
\hline 19 Sep 91 & 20 & 20.2400 & 8.2200 & 9.9100 \\
\hline 19 Sep 91 & 21 & 20.2800 & 8.2100 & 9.8700 \\
\hline 19 Sep 91 & 22 & 20.2300 & 8.1700 & 9.6000 \\
\hline $19 \operatorname{Sep} 91$ & 23 & 20.1200 & 8.1700 & 9.4700 \\
\hline 20 Sep 91 & 0 & 20.0900 & 8.1600 & 9.3600 \\
\hline 20 Sep 91 & 1 & 20.0500 & 8.1500 & 9.0900 \\
\hline 20 Sep 91 & 2 & 19.9700 & 8.1500 & 9.1200 \\
\hline 20 Sep 91 & 3 & 19.9300 & 8.1500 & 8.9700 \\
\hline 20 Sep 91 & 4 & 19.9000 & 8.1500 & 8.9700 \\
\hline 20 Sep 91 & 5 & 19.8700 & 8.1500 & 9.0300 \\
\hline 20 Sep 91 & 6 & 19.8500 & 8.1500 & 9.1700 \\
\hline 20 Sep 91 & 7 & 19.6900 & 8.1500 & 9.4500 \\
\hline 20 Sep 91 & 8 & 19.6800 & 8.1400 & 9.3800 \\
\hline 20 Sep 91 & 9 & 19.6600 & 8.1300 & 9.2500 \\
\hline 20 Sep 91 & 10 & 19.8100 & 8.1500 & 9.2000 \\
\hline 20 Sep 91 & 11 & 20.0400 & 8.2100 & 9.3500 \\
\hline 20 Sep 91 & 12 & 20.2300 & 8.2800 & 9.4400 \\
\hline $20 \operatorname{Sep} 91$ & 13 & 20.4200 & 8.3000 & 9.5100 \\
\hline 20 Sep 91 & 14 & 20.6400 & 8.2900 & 9.5000 \\
\hline $20 \operatorname{Sep} 91$ & 15 & 20.5500 & 8.3200 & 9.7200 \\
\hline 20 Sep 91 & 16 & 20.7300 & 8.2900 & 9.5200 \\
\hline $20 \operatorname{Sep} 91$ & 17 & 20.7000 & 8.2700 & 9.6100 \\
\hline 20 Sep 91 & 18 & 20.4900 & 8.2900 & 9.7200 \\
\hline 20 Sep 91 & 19 & 20.3600 & 8.2800 & 9.7100 \\
\hline 20 Sep 91 & 20 & 20.4100 & 8.3100 & 10.0300 \\
\hline
\end{tabular}


Table C.1 (continued)

\begin{tabular}{|c|c|c|c|c|}
\hline Date & Hour & $\begin{array}{l}\text { Temperature } \\
\left({ }^{\circ} \mathrm{C}\right)\end{array}$ & $\mathrm{pH}$ & $\begin{array}{c}\text { Dissolved } \\
\text { axygen } \\
(\mathrm{mg} / \mathrm{L})\end{array}$ \\
\hline 20 Sep 91 & 21 & 20.3900 & 8.3200 & 9.7900 \\
\hline 20 Sep 91 & 22 & 20.2200 & 8.2700 & 9.5900 \\
\hline 20 Sep 91 & 23 & 20.1200 & 8.2600 & 9.4900 \\
\hline $21 \operatorname{Sep} 91$ & 0 & 20.1500 & 8.2400 & 9.2900 \\
\hline 21 Sep 91 & 1 & 20.1300 & 8.2200 & 9.0800 \\
\hline 21 Sep 91 & 2 & 20.0900 & 8.2200 & 9.0400 \\
\hline 21 Sep 91 & 3 & 20.0700 & 8.2100 & 8.9200 \\
\hline 21 Sep 91 & 4 & 20.0400 & 8.2100 & 8.9100 \\
\hline 21 Sep 91 & 5 & 19.9900 & 8.1900 & 8.8900 \\
\hline 21 Sep 91 & 6 & 19.9900 & 8.1500 & 8.5500 \\
\hline 21 Sep 91 & 7 & 19.9600 & 8.1800 & 8.7100 \\
\hline 21 Sep 91 & 8 & 19.9100 & 8.2100 & 9.0200 \\
\hline 21 Sep 91 & 9 & 19.7600 & 8.2200 & 9.1900 \\
\hline 21 Sep 91 & 10 & 19.7300 & 8.2000 & 9.2500 \\
\hline 21 Sep 91 & 11 & 19.9500 & 8.2300 & 9.1400 \\
\hline 21 Sep 91 & 12 & 20.1400 & 8.2900 & 9.1700 \\
\hline 21 Sep 91 & 13 & 20.4900 & 8.3300 & 9.1100 \\
\hline 21 Sep 91 & 14 & 20.6800 & 8.3300 & 9.1900 \\
\hline 21 Sep 91 & 15 & 20.7800 & 8.2800 & 8.9500 \\
\hline 21 Sep 91 & 16 & 20.8300 & 8.2800 & 8.9800 \\
\hline 21 Sep 91 & 17 & 20.9700 & 8.2700 & 9.0400 \\
\hline $21 \operatorname{Sep} 91$ & 18 & 20.8600 & 8.3000 & 9.2300 \\
\hline 21 Sep 91 & 19 & 20.8200 & 8.2900 & 9.2300 \\
\hline 21 Sep 91 & 20 & 20.5600 & 8.2800 & 9.2600 \\
\hline 21 Sep 91 & 21 & 20.2100 & 8.2300 & 9.0700 \\
\hline 21 Sep 91 & 22 & 19.9600 & 8.2100 & 9.0000 \\
\hline 21 Sep 91 & 23 & 19.9900 & 8.1700 & 8.6900 \\
\hline 22 Sep 91 & 0 & 19.9400 & 8.1700 & 8.6600 \\
\hline $22 \operatorname{Sep} 91$ & 1 & 19.9400 & 8.1500 & 8.4400 \\
\hline $22 \operatorname{Sep} 91$ & 2 & 19.8900 & 8.1600 & 8.5300 \\
\hline $22 \operatorname{Sep} 91$ & 3 & 19.8500 & 8.1600 & 8.5000 \\
\hline $22 \operatorname{Sep} 91$ & 4 & 19.8300 & 8.1500 & 8.4200 \\
\hline
\end{tabular}




\section{C-42}

Table C.1 (continued)

\begin{tabular}{|c|c|c|c|c|}
\hline Date & Hour & $\begin{array}{c}\text { Temperature } \\
\left({ }^{\circ} \mathrm{C}\right)\end{array}$ & $\mathrm{pH}$ & $\begin{array}{l}\text { Dissolved } \\
\text { axygen } \\
(\mathrm{mg} / \mathrm{L})\end{array}$ \\
\hline $22 \operatorname{Sep} 91$ & 5 & 19.7200 & 8.1700 & 8.6500 \\
\hline 22 Sep 91 & 6 & 19.7500 & 8.1600 & 8.4500 \\
\hline $22 \operatorname{Sep} 91$ & 7 & 19.7000 & 8.1700 & 8.5600 \\
\hline $22 \operatorname{Sep} 91$ & 8 & 19.7500 & 8.1500 & 8.3700 \\
\hline $22 \operatorname{Sep} 91$ & 9 & 19.6900 & 8.1900 & 8.6600 \\
\hline 22 Sep 91 & 10 & 19.6300 & 8.2100 & 8.8000 \\
\hline $22 \operatorname{Sep} 91$ & 11 & 19.6100 & 8.2000 & 8.6600 \\
\hline 22 Sep 91 & 12 & 19.8000 & 8.2200 & 8.6000 \\
\hline $22 \operatorname{Sep} 91$ & 13 & 20.0600 & 8.2100 & 8.5900 \\
\hline 22 Sep 91 & 14 & 20.9300 & 8.1800 & 8.2100 \\
\hline $22 \operatorname{Sep} 91$ & 15 & 21.0400 & 8.2000 & 8.3100 \\
\hline $22 \operatorname{Sep} 91$ & 16 & 21.2300 & 8.2300 & 8.4600 \\
\hline 10 Feb 92 & 17 & 5.8600 & 9.5000 & 11.3400 \\
\hline 10 Feb 92 & 18 & 5.5800 & 9.2500 & 11.0700 \\
\hline 10 Feb 92 & 19 & 5.8200 & 8.5400 & 10.9700 \\
\hline 10 Feb 92 & 20 & 5.5900 & 8.4100 & 11.0100 \\
\hline 10 Feb 92 & 21 & 5.5600 & 8.3300 & 11.0900 \\
\hline 10 Feb 92 & 22 & 5.5800 & 8.3000 & 11.0500 \\
\hline 10 Feb 92 & 23 & 5.6200 & 8.3000 & 11.1000 \\
\hline 11 Feb 92 & 0 & 5.6700 & 8.3000 & 11.2700 \\
\hline 11 Feb 92 & 1 & 5.7500 & 8.2900 & 11.2700 \\
\hline 11 Feb 92 & 2 & 5.8400 & 8.2900 & 11.3200 \\
\hline 11 Feb 92 & 3 & 5.9100 & 8.3000 & 11.3900 \\
\hline 11 Feb 92 & 4 & 5.9500 & 8.3000 & 11.4500 \\
\hline 11 Feb 92 & 5 & 5.9900 & 8.3100 & 11.5000 \\
\hline 11 Feb 92 & 6 & 6.0400 & 8.3300 & 11.5700 \\
\hline 11 Feb 92 & 7 & 6.1500 & 8.3200 & 11.5800 \\
\hline 11 Feb 92 & 8 & 6.2400 & 8.3200 & 11.7100 \\
\hline 11 Feb 92 & 9 & 6.4900 & 8.3700 & 11.6200 \\
\hline 11 Feb 92 & 10 & 6.5800 & 8.3700 & 11.7300 \\
\hline 11 Feb 92 & 11 & 6.6200 & 8.3700 & 11.7200 \\
\hline 11 Feb 92 & 12 & 6.6000 & 8.3900 & 11.7300 \\
\hline
\end{tabular}


Table C.1 (continued)

\begin{tabular}{|c|c|c|c|c|}
\hline Date & Hour & $\begin{array}{c}\text { Temperature } \\
\left({ }^{\circ} \mathrm{C}\right)\end{array}$ & pH & $\begin{array}{c}\text { Dissolved } \\
\text { axygen } \\
(\mathrm{mg} / \mathrm{L})\end{array}$ \\
\hline 11 Feb 92 & 13 & 6.5300 & 8.4200 & 11.8700 \\
\hline 11 Feb 92 & 14 & 6.5100 & 8.4300 & 11.6100 \\
\hline 11 Feb 92 & 15 & 6.4400 & 8.4200 & 11.5500 \\
\hline 11 Feb 92 & 16 & 6.4400 & 8.4100 & 11.5100 \\
\hline 11 Feb 92 & 17 & 6.4300 & 8.4100 & 11.5400 \\
\hline 11 Feb 92 & 18 & 13.1800 & 8.9200 & 10.0800 \\
\hline 11 Feb 92 & 19 & 5.8600 & 8.5300 & 10.9600 \\
\hline 11 Feb 92 & 20 & 5.5800 & 8.4100 & 11.0900 \\
\hline 11 Feb 92 & 21 & 5.5600 & 8.3300 & 11.0700 \\
\hline 11 Feb 92 & 22 & 5.5800 & 8.3000 & 11.0900 \\
\hline 11 Feb 92 & 23 & 5.6200 & 8.2900 & 11.1800 \\
\hline 12 Feb 92 & 0 & & & \\
\hline 13 Feb 92 & 10 & 22.4700 & 4.9900 & 6.3800 \\
\hline 13 Feb 92 & 13 & 8.3400 & 8.1800 & 22.0800 \\
\hline 13 Feb 92 & 14 & 8.3600 & 8.3000 & 14.6500 \\
\hline 13 Feb 92 & 15 & 8.4500 & 8.4000 & 13.2400 \\
\hline 13 Feb 92 & 16 & 8.4100 & 8.6100 & 17.1900 \\
\hline 13 Feb 92 & 17 & 8.3700 & 8.3500 & 17.0900 \\
\hline 13 Feb 92 & 18 & 8.4900 & 8.2700 & 17.2800 \\
\hline 13 Feb 92 & 19 & 8.5600 & 8.2300 & 17.4300 \\
\hline 13 Feb 92 & 20 & 8.5300 & 8.2300 & 17.4600 \\
\hline 13 Feb 92 & 21 & 8.4300 & 8.2200 & 17.3100 \\
\hline 13 Feb 92 & 22 & 8.4200 & 8.2200 & 17.1500 \\
\hline 13 Feb 92 & 23 & 8.4300 & 8.1700 & 16.6300 \\
\hline 14 Feb 92 & 0 & 8.5100 & 8.1300 & 16.1300 \\
\hline 14 Feb 92 & 1 & 8.5400 & 8.1300 & 16.0900 \\
\hline 14 Feb 92 & 2 & 8.6200 & 8.1100 & 15.8900 \\
\hline 14 Feb 92 & 3 & 8.6600 & 8.1100 & 15.8300 \\
\hline 14 Feb 92 & 4 & 8.7200 & 8.1100 & 15.7900 \\
\hline 14 Feb 92 & 5 & 8.7400 & 8.1400 & 15.8800 \\
\hline 14 Feb 92 & 6 & 8.7500 & 8.1400 & 15.9100 \\
\hline 14 Feb 92 & 7 & 8.6800 & 8.1400 & 16.3700 \\
\hline
\end{tabular}


Table C.1 (continued)

\begin{tabular}{|c|c|c|c|c|}
\hline Date & Hour & $\begin{array}{l}\text { Temperature } \\
\left({ }^{\circ} \mathrm{C}\right)\end{array}$ & $\mathrm{pH}$ & $\begin{array}{c}\text { Dissolved } \\
\text { axygen } \\
(\mathrm{mg} / \mathrm{L})\end{array}$ \\
\hline 14 Feb 92 & 8 & 8.5200 & 8.1700 & 16.7600 \\
\hline 14 Feb 92 & 9 & 8.4100 & 8.2000 & 16.9700 \\
\hline 14 Feb 92 & 10 & 8.4600 & 8.2000 & 16.8800 \\
\hline 14 Feb 92 & 11 & 8.5000 & 8.1800 & 16.9000 \\
\hline 14 Feb 92 & 12 & 8.5200 & 8.1700 & 16.8300 \\
\hline 14 Feb 92 & 13 & 8.6000 & 8.1300 & 16.7400 \\
\hline 14 Feb 92 & 14 & 9.1100 & 8.4300 & 15.4900 \\
\hline 14 Feb 92 & 15 & 9.2400 & 8.2200 & 15.2200 \\
\hline 14 Feb 92 & 16 & 9.3100 & 8.8300 & 15.2800 \\
\hline 14 Feb 92 & 17 & 9.4900 & 8.7000 & 14.8800 \\
\hline 14 Feb 92 & 18 & 9.6300 & 8.1000 & 14.6600 \\
\hline 14 Feb 92 & 19 & 9.6600 & 8.0200 & 14.6200 \\
\hline 14 Feb 92 & 20 & 9.3800 & 8.1200 & 15.4600 \\
\hline 14 Feb 92 & 21 & 9.6000 & 8.0500 & 15.1800 \\
\hline 14 Feb 92 & 22 & 8.8600 & 8.1800 & 16.5800 \\
\hline 14 Feb 92 & 23 & 9.2300 & 8.1600 & 16.1300 \\
\hline 15 Feb 92 & 0 & 8.8300 & 8.2300 & 16.7400 \\
\hline 15 Feb 92 & 1 & 8.8100 & 8.2400 & 16.7100 \\
\hline 15 Feb 92 & 2 & 8.7900 & 8.2600 & 16.7100 \\
\hline 15 Feb 92 & 3 & 8.8200 & 8.2400 & 16.6700 \\
\hline 15 Feb 92 & 4 & 9.0500 & 8.2000 & 16.2800 \\
\hline 15 Feb 92 & 5 & 9.2500 & 8.1700 & 16.0800 \\
\hline 15 Feb 92 & 6 & 8.8800 & 8.2200 & 16.4000 \\
\hline 15 Feb 92 & 7 & 8.8400 & 8.2000 & 16.4800 \\
\hline 15 Feb 92 & 8 & 9.3200 & 8.1100 & 15.7600 \\
\hline 15 Feb 92 & 9 & 9.7400 & 8.0300 & 15.0400 \\
\hline 15 Feb 92 & 10 & 9.2600 & 8.0600 & 15.8400 \\
\hline 15 Feb 92 & 11 & 9.0800 & 8.1000 & 15.8500 \\
\hline 15 Feb 92 & 12 & 9.9100 & 7.8700 & 14.8000 \\
\hline 15 Feb 92 & 13 & 9.8200 & 7.8800 & 14.4900 \\
\hline 15 Feb 92 & 14 & 9.4500 & 7.9600 & 15.2900 \\
\hline 15 Feb 92 & 15 & 10.0700 & 7.8700 & 14.7500 \\
\hline
\end{tabular}




\section{C-45}

Table C.1 (continued)

\begin{tabular}{|c|c|c|c|c|}
\hline Date & Hour & $\begin{array}{c}\text { Temperature } \\
\left({ }^{\circ} \mathrm{C}\right) \\
\end{array}$ & $\mathrm{pH}$ & $\begin{array}{c}\text { Dissolved } \\
\text { axygen } \\
(\mathrm{mg} / \mathrm{L})\end{array}$ \\
\hline 15 Feb 92 & 16 & 10.4100 & 7.8700 & 14.1900 \\
\hline 15 Feb 92 & 17 & 10.4300 & 7.9100 & 14.6100 \\
\hline 15 Feb 92 & 18 & 10.4600 & 7.9400 & 14.7200 \\
\hline 15 Feb 92 & 19 & 11.3300 & 7.8000 & 13.7100 \\
\hline 15 Feb 92 & 20 & 11.5100 & 7.7800 & 13.5100 \\
\hline 15 Feb 92 & 21 & 10.9600 & 7.8900 & 14.0400 \\
\hline 15 Feb 92 & 22 & 9.7700 & 8.0500 & 15.3400 \\
\hline 15 Feb 92 & 23 & 10.2500 & 7.9900 & 15.0900 \\
\hline 16 Feb 92 & 0 & 10.8600 & 7.9500 & 14.4900 \\
\hline 16 Feb 92 & 1 & 10.9400 & 7.8900 & 14.2900 \\
\hline 16 Feb 92 & 2 & 10.6900 & 7.9000 & 14.2800 \\
\hline 16 Feb 92 & 3 & 10.5200 & 7.9000 & 14.4700 \\
\hline 16 Feb 92 & 4 & 10.6900 & 7.8700 & 14.2400 \\
\hline 16 Feb 92 & 5 & 10.6100 & 7.8900 & 14.1600 \\
\hline 16 Feb 92 & 6 & 10.5000 & 7.8700 & 14.3100 \\
\hline 16 Feb 92 & 7 & 10.5000 & 7.8700 & 14.3300 \\
\hline 16 Feb 92 & 8 & 10.9200 & 7.7900 & 13.7400 \\
\hline 16 Feb 92 & 9 & 11.2400 & 7.7300 & 13.0400 \\
\hline 16 Feb 92 & 10 & 10.9300 & 7.7600 & 13.3000 \\
\hline 16 Feb 92 & 11 & 9.8500 & 7.9000 & 14.8500 \\
\hline 16 Feb 92 & 12 & 9.8800 & 7.8900 & 15.1200 \\
\hline 16 Feb 92 & 13 & 10.5600 & 7.8400 & 14.2600 \\
\hline 16 Feb 92 & 14 & 10.8700 & 7.8300 & 14.1500 \\
\hline 16 Feb 92 & 15 & 9.9900 & 7.9600 & 15.1900 \\
\hline 16 Feb 92 & 16 & 9.9900 & 7.9800 & 15.3900 \\
\hline 16 Feb 92 & 17 & 9.1300 & 8.0800 & $16.180 \mathrm{~J}$ \\
\hline 16 Feb 92 & 18 & 10.5000 & 7.9700 & 15,5700 \\
\hline 16 Feb 92 & 19 & 11.3700 & 7.9200 & 14.3900 \\
\hline 16 Feb 92 & 20 & 11.8500 & 7.8500 & 13.9600 \\
\hline 16 Feb 92 & 21 & 11.6000 & 7.8300 & 14.2200 \\
\hline 16 Feb 92 & 22 & 11.0000 & 7.8700 & 14.4200 \\
\hline 16 Feb 92 & 23 & 9.2800 & 8.0500 & 16.0300 \\
\hline
\end{tabular}




\section{C-46}

Table C.1 (continued)

\begin{tabular}{|c|c|c|c|c|}
\hline Date & Hour & $\begin{array}{c}\text { Temperature } \\
\left({ }^{\circ} \mathrm{C}\right)\end{array}$ & $\mathrm{pH}$ & $\begin{array}{c}\text { Dissolved } \\
\text { oxygen } \\
(\mathrm{mg} / \mathrm{L})\end{array}$ \\
\hline 17 Feb 92 & 0 & 9.1900 & 8.0600 & 15.8600 \\
\hline 17 Feb 92 & 1 & 9.1900 & 8.0600 & 15.8400 \\
\hline 17 Feb 92 & 2 & 8.4000 & 8.1200 & 16.7300 \\
\hline 17 Feb 92 & 3 & 8.8000 & 8.0300 & 16.1400 \\
\hline 17 Feb 92 & 4 & 8.6800 & 8.0000 & 16.0300 \\
\hline 17 Feb 92 & 5 & 8.7600 & 7.9600 & 16.1000 \\
\hline 17 Feb 92 & 6 & 9.7900 & 7.7600 & 14.7500 \\
\hline $17 \mathrm{Feb} 92$ & 7 & 8.6000 & 7.9400 & 15.6000 \\
\hline 17 Feb 92 & 8 & 8.1200 & 8.0500 & 17.1200 \\
\hline 17 Feb 92 & 9 & 8.1800 & 8.1000 & 17.0800 \\
\hline 17 Feb 92 & 10 & 8.3100 & 8.1100 & 16.9100 \\
\hline 17 Feb 92 & 11 & 9.1200 & 7.9000 & 13.9900 \\
\hline 17 Feb 92 & 12 & 9.8000 & 7.6800 & 14.0300 \\
\hline 17 Feb 92 & 13 & 10.0600 & 7.6600 & 13.6100 \\
\hline 17 Feb 92 & 14 & 9.6100 & 7.8000 & 14.5400 \\
\hline 17 Feb 92 & 15 & 9.2300 & 7.9200 & 15.3200 \\
\hline 17 Feb 92 & 16 & 9.4600 & 7.8800 & 15.1400 \\
\hline 17 Feb 92 & 17 & 8.8400 & 8.0400 & 16.1400 \\
\hline 17 Feb 92 & 18 & 9.4100 & 7.9100 & 15.4900 \\
\hline 17 Feb 92 & 19 & 9.6800 & 7.9100 & 14.8100 \\
\hline 17 Feb 92 & 20 & 9.7900 & 7.8700 & 14.8600 \\
\hline 17 Feb 92 & 21 & 9.5400 & 7.8400 & 14.9500 \\
\hline 17 Feb 92 & 22 & 9.0300 & 8.0300 & 15.9500 \\
\hline 17 Feb 92 & 23 & 9.3300 & 7.9500 & 15.6700 \\
\hline 18 Feb 92 & 0 & 8.5600 & 8.0700 & 16.5000 \\
\hline 18 Feb 92 & 0 & & & \\
\hline 18 Feb 92 & 1 & 8.3500 & 8.1200 & 16.8900 \\
\hline 18 Feb 92 & 2 & 8.3500 & 8.1200 & 16.8500 \\
\hline 18 Feb 92 & 3 & 8.3500 & 8.0600 & 16.8000 \\
\hline 18 Feb 92 & 4 & 8.4200 & 8.0200 & 17.1200 \\
\hline 19 Feb 92 & 0 & & & \\
\hline 24 Feb 92 & 15 & 12.5000 & 8.1100 & 12.5400 \\
\hline
\end{tabular}


Table C.1 (continued)

\begin{tabular}{|c|c|c|c|c|}
\hline Date & Hour & $\begin{array}{l}\text { Temperature } \\
\left({ }^{\circ} \mathrm{C}\right)\end{array}$ & $\mathrm{pH}$ & $\begin{array}{l}\text { Dissolved } \\
\text { axygen } \\
\text { (mg/L) }\end{array}$ \\
\hline 24 Feb 92 & 16 & 12.6300 & 8.4900 & 10.3800 \\
\hline 24 Feb 92 & 17 & 12.8800 & 7.9300 & 10.2500 \\
\hline 24 Feb 92 & 18 & 13.1100 & 7.9000 & 10.1400 \\
\hline 24 Feb 92 & 19 & 13.2100 & 7.8900 & 10.0900 \\
\hline 24 Feb 92 & 20 & 13.2900 & 7.8900 & 10.0600 \\
\hline 24 Feb 92 & 21 & 13.3400 & 7.8800 & 9.9900 \\
\hline 24 Feb 92 & 22 & 13.3600 & 7.8900 & 9.9900 \\
\hline 24 Feb 92 & 23 & 13.4200 & 7.8800 & 9.9900 \\
\hline 25 Feb 92 & 0 & 13.3800 & 7.8700 & 10.0000 \\
\hline 25 Feb 92 & 1 & 13.2200 & 7.8700 & 9.9600 \\
\hline 25 Feb 92 & 2 & 13.0400 & 7.8600 & 9.9700 \\
\hline 25 Feb 92 & 3 & 12.9500 & 7.8400 & 9.9400 \\
\hline 25 Feb 92 & 4 & 12.8900 & 7.8100 & 9.9700 \\
\hline 25 Feb 92 & 5 & 12.7400 & 7.7800 & 9.9800 \\
\hline 25 Feb 92 & 6 & 12.5700 & 7.7700 & 9.9700 \\
\hline 25 Feb 92 & 7 & 12.4200 & 7.7600 & 9.9500 \\
\hline 25 Feb 92 & 8 & 12.2900 & 7.7500 & 9.9000 \\
\hline 25 Feb 92 & 9 & 12.2500 & 7.7900 & 9.8700 \\
\hline 25 Feb 92 & 10 & 12.2800 & 7.7600 & 9.8900 \\
\hline 25 Feb 92 & 11 & 12.3400 & 7.7800 & 9.8700 \\
\hline 25 Feb 92 & 12 & 12.3400 & 7.7600 & 9.8000 \\
\hline 25 Feb 92 & 13 & 12.3100 & 7.7500 & 9.7200 \\
\hline 25 Feb 92 & 14 & 12.3000 & 7.7400 & 9.7200 \\
\hline 25 Feb 92 & 15 & 12.3000 & 7.7400 & 9.7000 \\
\hline 25 Feb 92 & 16 & 12.2800 & 7.7400 & 9.6800 \\
\hline 25 Feb 92 & 17 & 12.2800 & 7.7300 & 9.6200 \\
\hline 25 Feb 92 & 18 & 12.1600 & 7.7700 & 9.6500 \\
\hline 25 Feb 92 & 19 & 12.1500 & 7.7600 & 9.7300 \\
\hline 25 Feb 92 & 20 & 12.4400 & 7.6600 & 9.9900 \\
\hline 25 Feb 92 & 21 & 12.1500 & 7.7300 & 9.7000 \\
\hline 25 Feb 92 & 22 & 12.3100 & 7.6400 & 9.5300 \\
\hline 25 Feb 92 & 23 & 12.4100 & 7.6500 & 9.7200 \\
\hline
\end{tabular}




\section{C-48}

Table C.1 (continued)

\begin{tabular}{|c|c|c|c|c|}
\hline Date & Hour & $\begin{array}{c}\text { Temperature } \\
\left({ }^{\circ} \mathrm{C}\right)\end{array}$ & $\mathrm{pH}$ & $\begin{array}{c}\text { Dissolved } \\
\text { axygen } \\
(\mathrm{mg} / \mathrm{L})\end{array}$ \\
\hline 26 Feb 92 & 0 & 12.4700 & 7.7100 & 9.7900 \\
\hline 26 Feb 92 & 1 & 12.4500 & 7.7200 & 9.8300 \\
\hline 26 Feb 92 & 2 & 12.4400 & 7.7300 & 9.8400 \\
\hline 26 Feb 92 & 3 & 12.3300 & 7.7700 & 9.8200 \\
\hline 26 Feb 92 & 4 & 12.2600 & 7.7300 & 9.7800 \\
\hline 26 Feb 92 & 5 & 11.9500 & 7.7300 & 9.8900 \\
\hline 26 Feb 92 & 6 & 11.9400 & 7.6500 & 9.8700 \\
\hline 26 Feb 92 & 7 & 11.8000 & 7.6200 & 9.9000 \\
\hline 26 Feb 92 & 8 & 11.6600 & 7.6000 & 9.9200 \\
\hline 26 Feb 92 & 9 & 11.4600 & 7.5800 & 10.0700 \\
\hline 26 Feb 92 & 10 & 11.2700 & 7.4500 & 17.7200 \\
\hline 26 Feb 92 & 11 & 11.1400 & 7.3800 & 18.0000 \\
\hline 26 Feb 92 & 12 & 11.0400 & 7.3800 & 18.1600 \\
\hline 26 Feb 92 & 13 & 10.9400 & 7.3700 & 18.2800 \\
\hline 26 Feb 92 & 14 & 10.9200 & 7.3400 & 18.2900 \\
\hline 26 Feb 92 & 15 & 10.8300 & 7.3400 & 18.3900 \\
\hline 26 Feb 92 & 16 & 10.6900 & 7.4100 & 18.1600 \\
\hline 26 Feb 92 & 17 & 10.5900 & 7.3500 & 18.7000 \\
\hline 26 Feb 92 & 18 & 10.5200 & 7.3600 & 18.7500 \\
\hline $26 \mathrm{Feb} 92$ & 19 & 10.4500 & 7.3300 & 19.1500 \\
\hline 26 Feb 92 & 20 & 10.3400 & 7.2900 & 19.3800 \\
\hline 26 Feb 92 & 21 & 10.1700 & 7.3500 & 19.0100 \\
\hline 26 Feb 92 & 22 & 10.0800 & 7.3100 & 18.8900 \\
\hline $26 \mathrm{Feb} 92$ & 23 & 9.9700 & 7.3400 & 19.1300 \\
\hline 27 Feb 92 & 0 & 9.8700 & 7.3800 & 19.3000 \\
\hline 27 Feb 92 & 1 & 9.8000 & 7.4000 & 19.3300 \\
\hline 27 Feb 92 & 2 & 9.7200 & 7.4100 & 19.3800 \\
\hline $27 \mathrm{Feb} 92$ & 3 & 9.6800 & 7.9900 & 19.6200 \\
\hline 27 Feb 92 & 4 & 9.6300 & 7.3200 & 20.1800 \\
\hline 27 Feb 92 & 5 & 9.5400 & 7.3100 & 20.2800 \\
\hline 27 Feb 92 & 6 & 9.4300 & 7.3100 & 20.3000 \\
\hline 27 Feb 92 & 7 & 9.3100 & 7.3300 & 20.3200 \\
\hline
\end{tabular}


Table C.1 (continued)

\begin{tabular}{|c|c|c|c|c|}
\hline Date & Hour & $\begin{array}{c}\text { Temperature } \\
\left.i^{\circ} \mathrm{C}\right)\end{array}$ & $\mathrm{pH}$ & $\begin{array}{c}\text { Dissolved } \\
\text { axygen } \\
(\mathrm{mg} / \mathrm{L})\end{array}$ \\
\hline 27 Feb 92 & 8 & 9.5500 & 7.8400 & 20.6000 \\
\hline 27 Feb 92 & 9 & 9.2700 & 7.5400 & 20.9000 \\
\hline 27 Feb 92 & 10 & 9.0300 & 7.3300 & 20.3700 \\
\hline 27 Feb 92 & 11 & 9.2800 & 7.5300 & 19.4900 \\
\hline 27 Feb 92 & 12 & 9.3900 & 7.5300 & 19.3700 \\
\hline 27 Feb 92 & 13 & 9.3500 & 7.3500 & 19.6100 \\
\hline 27 Feb 92 & 14 & 9.5400 & 7.3300 & 19.4700 \\
\hline 27 Feb 92 & 15 & 9.7900 & 7.3800 & 19.1600 \\
\hline 27 Feb 92 & 16 & 10.0800 & 7.4100 & 18.8700 \\
\hline 27 Feb 92 & 17 & 10.3200 & 7.4100 & 13.6700 \\
\hline 27 Feb 92 & 18 & 10.5000 & 7.4500 & $18.79 \mathrm{~cm}$ \\
\hline 27 Feb 92 & 19 & 10.2900 & 7.9900 & 19.5900 \\
\hline 27 Fcb 92 & 20 & 10.0900 & 8.0600 & 20.5400 \\
\hline 27 Feb 92 & 21 & 10.1200 & 7.9800 & 20.5400 \\
\hline 27 Feb 92 & 22 & 10.4000 & 7.5300 & 19.2300 \\
\hline 27 Feb 92 & 23 & 10.2900 & 7.5400 & 18.7500 \\
\hline 28 Feb 92 & 0 & 10.1200 & 7.5000 & 18.8500 \\
\hline 28 Feb 92 & 1 & 10.0100 & 7.4400 & 19.1900 \\
\hline 28 Feb 92 & 2 & 9.9500 & 7.4300 & 19.3600 \\
\hline 28 Feb 92 & 3 & 9.9100 & 7.4400 & $19 .+100$ \\
\hline 28 Feb 92 & 4 & 9.8800 & 7.4500 & 19.3900 \\
\hline 28 Feb 92 & 5 & 9.8400 & 7.4400 & 19.3000 \\
\hline 28 Feb 92 & 6 & 9.7900 & 7.4500 & 19.2400 \\
\hline 28 Feb 92 & 7 & 9.8200 & 7.9200 & 20.1800 \\
\hline 28 Feb 92 & 8 & 9.7700 & 8.0500 & 20.7700 \\
\hline 28 Feb 92 & 9 & 9.8700 & 8.0900 & 20.7800 \\
\hline 28 Feb 92 & 10 & 9.7400 & 7.7200 & 19.9700 \\
\hline 28 Feb 92 & 11 & 9.8400 & 7.5000 & 19.2200 \\
\hline 28 Feb 92 & 12 & 9.9800 & 7.5000 & 18.9600 \\
\hline 28 Feb 92 & 13 & 10.1600 & 7.4900 & 18.9800 \\
\hline $02 \operatorname{Mar} 92$ & 10 & 10.6900 & 7.5600 & 18.5700 \\
\hline 02 Mar 92 & 11 & 10.6300 & 7.3900 & 18.2100 \\
\hline
\end{tabular}


Table C.1 (continued)

\begin{tabular}{|c|c|c|c|c|}
\hline Date & Hour & $\begin{array}{c}\text { Temperature } \\
\left({ }^{\circ} \mathrm{C}\right)\end{array}$ & $\mathrm{pH}$ & $\begin{array}{c}\text { Dissolved } \\
\text { oxygen } \\
(\mathrm{mg} / \mathrm{L})\end{array}$ \\
\hline $02 \operatorname{Mar} 92$ & 12 & 10.6500 & 7.3700 & 18.0800 \\
\hline $02 \operatorname{Mar} 92$ & 13 & 10.8600 & 7.4500 & 17.9900 \\
\hline 02 Mar 92 & 14 & 11.4400 & 7.5700 & 17.4500 \\
\hline 02 Mar 92 & 15 & 11.5700 & 7.5800 & 17.1400 \\
\hline $02 \operatorname{Mar} 92$ & 16 & 12.0100 & 7.5900 & 16.8500 \\
\hline 02 Mar 92 & 17 & 11.8400 & 7.4700 & 16.9500 \\
\hline 02 Mar 92 & 18 & 11.9100 & 7.5200 & 16.8900 \\
\hline 02 Mar 92 & 19 & 11.8800 & 7.5800 & 16.8700 \\
\hline $02 \operatorname{Mar} 92$ & 20 & 12.1500 & 7.5700 & 16.6700 \\
\hline 02 Mar 92 & 21 & 12.3700 & 7.5700 & 16.4700 \\
\hline $02 \operatorname{Mar} 92$ & 22 & 12.3300 & 7.5900 & 16.4600 \\
\hline 02 Mar 92 & 23 & 12.1400 & 7.6100 & 16.5800 \\
\hline 03 Mar 92 & 0 & 11.9300 & 7.6500 & 16.7200 \\
\hline 03 Mar 92 & 1 & 11.7300 & 7.6500 & 16.8900 \\
\hline $03 \operatorname{Mar} 92$ & 2 & 11.7500 & 7.6000 & 16.8900 \\
\hline $03 \operatorname{Mar} 92$ & 3 & 11.7500 & 7.5600 & 16.8800 \\
\hline 03 Mat 92 & 4 & 11.6600 & 7.5400 & $16.96 \mathrm{v}$ \\
\hline C3 hatar 92 & 5 & 11.4300 & 7.5400 & 17.2100 \\
\hline 03 Mar 92 & 6 & 11.4500 & 7.5200 & 17.2900 \\
\hline 03 Mar 92 & 7 & 11.3300 & 7.4900 & 17.7900 \\
\hline 03 Mar 92 & 8 & 11.1100 & 7.4800 & 18.2500 \\
\hline 03 Mar 96 & 9 & 10.9800 & 7.5900 & 18.3000 \\
\hline 03 Mar 92 & 10 & 10.9200 & 7.6900 & 18.2600 \\
\hline 03 Mar 92 & 11 & 11.1100 & 7.5800 & 17.6000 \\
\hline 03 Mar 92 & 12 & 11.3800 & 7.5100 & 17.0000 \\
\hline 03 Mar 92 & 13 & 11.5500 & 7.4800 & 16.8700 \\
\hline 03 Mar 92 & 14 & 11.7900 & 7.4500 & 16.8200 \\
\hline 03 Mar 92 & 15 & 11.9900 & 7.4800 & 16.6200 \\
\hline 03 Mar 92 & 16 & 11.8700 & 7.6700 & 16.5400 \\
\hline 03 Mar 92 & 17 & 12.2900 & 7.5900 & 16.1600 \\
\hline 03 Mar 92 & 18 & 12.1300 & 7.7500 & 16.1900 \\
\hline 03 Mar 92 & 19 & 12.1400 & 7.8000 & 16.1800 \\
\hline
\end{tabular}




\section{C-51}

Table C.1 (continued)

\begin{tabular}{|c|c|c|c|c|}
\hline Date & Hour & $\begin{array}{c}\text { Temperature } \\
\left({ }^{\circ} \mathrm{C}\right)\end{array}$ & $\mathrm{pH}$ & $\begin{array}{c}\text { Dissolved } \\
\text { axygen } \\
(\mathrm{mg} / \mathrm{L}) \\
\end{array}$ \\
\hline 03 Mar 92 & 20 & 11.9400 & 7.8400 & 16.3000 \\
\hline 03 Mar 92 & 21 & 12.1300 & 7.8300 & 16.1400 \\
\hline 03 Mar 92 & 22 & 12.3700 & 7.7900 & 15.9400 \\
\hline 03 Mar 92 & 23 & 12.0800 & 7.8300 & 16.1000 \\
\hline $04 \mathrm{Mar} 92$ & 0 & 11.9600 & 7.8200 & 16.1900 \\
\hline 04 Mar 92 & 1 & 12.1700 & 7.7700 & 16.0400 \\
\hline 04 Mar 92 & 2 & 12.2700 & 7.7600 & 15.9300 \\
\hline 04 Mar 92 & 3 & 12.2700 & 7.7800 & 15.9500 \\
\hline 04 Mar 92 & 4 & 12.3600 & 7.7900 & 15.8700 \\
\hline 04 Mar 92 & 5 & 12.3000 & 7.7500 & 16.0000 \\
\hline 04 Mar 92 & 6 & 12.1300 & 7.7600 & 16.1300 \\
\hline 04 Mar 92 & 7 & 11.8800 & 7.7900 & 16.3400 \\
\hline 04 Mar 92 & 8 & 11.9800 & 7.8000 & 16.6000 \\
\hline 04 Mar 92 & 9 & 12.1300 & 7.8400 & 16.7500 \\
\hline 04 Mar 92 & 10 & 12.2100 & 7.7500 & 16.4300 \\
\hline $04 \operatorname{Mar} 92$ & 11 & 12.1200 & 7.6800 & 16.6100 \\
\hline 04 Mar 92 & 12 & 12.4300 & 7.6700 & 15.9900 \\
\hline 04 Mar 92 & 13 & 12.3300 & 7.6800 & 15.6700 \\
\hline 04 Mar 92 & 14 & 12.6500 & 7.6500 & 15.4400 \\
\hline 04 Mar 92 & 15 & 12.4800 & 7.7700 & 15.4800 \\
\hline 04 Mar 92 & 16 & 12.1300 & 7.8200 & 15.8700 \\
\hline 04 Mar 92 & 17 & 11.9600 & 7.8600 & 15.9400 \\
\hline 04 Mar 92 & 18 & 11.8500 & 7.8500 & 15.9700 \\
\hline 04 Mar 92 & 19 & 11.9200 & 7.8600 & 15.9300 \\
\hline 04 Mar 92 & 20 & 12.0700 & 7.8500 & 15.8000 \\
\hline 04 Mar 92 & 21 & 12.1600 & 7.8400 & 15.7400 \\
\hline 04 Mar 92 & 22 & 12.1500 & 7.8200 & 15.7300 \\
\hline 04 Mar 92 & 23 & 12.2700 & 7.8100 & 15.6400 \\
\hline 05 Mar 92 & 0 & 12.3400 & 7.8000 & 15.5800 \\
\hline 05 Mar 92 & 1 & 12.5100 & 7.7600 & 15.4100 \\
\hline 05 Mar 92 & 2 & 12.5000 & 7.7500 & 15.4300 \\
\hline 05 Mar 92 & 3 & 12.2700 & 7.7500 & 15.6300 \\
\hline
\end{tabular}


Table C.1 (continued)

\begin{tabular}{|c|c|c|c|c|}
\hline Date & Hour & $\begin{array}{c}\text { Temperature } \\
\left({ }^{\circ} \mathrm{C}\right)\end{array}$ & $\mathrm{pH}$ & $\begin{array}{c}\text { Dissolved } \\
\text { axygen } \\
(\mathrm{mg} / \mathrm{L})\end{array}$ \\
\hline 05 Mar 92 & 4 & 12.1700 & 7.7300 & 15.7400 \\
\hline 05 Mar 92 & 5 & 12.1200 & 7.7100 & 15.8200 \\
\hline $05 \operatorname{Mar} 92$ & 6 & 12.1200 & 7.6900 & 15.8400 \\
\hline 05 Mar 92 & 7 & 11.9900 & 7.6200 & 15.9400 \\
\hline 05 Mar 92 & 8 & 11.9000 & 7.7000 & 15.4500 \\
\hline 05 Mar 92 & 9 & 11.9500 & 7.7700 & 15.9100 \\
\hline 05 Mar 92 & 10 & 12.2400 & 7.7200 & 15.9400 \\
\hline 05 Mar 92 & 11 & 12.8300 & 7.6400 & 15.7300 \\
\hline 05 Mar 92 & 12 & 12.6500 & 7.6800 & 15.8700 \\
\hline 05 Mar 92 & 13 & 13.0100 & 7.6500 & 15.0200 \\
\hline 05 Mar 92 & 14 & 13.3600 & 7.6600 & 14.5600 \\
\hline 05 Mar 92 & 15 & 13.5100 & 7.7000 & 14.5200 \\
\hline $05 \operatorname{Mar} 92$ & 16 & 13.4200 & 7.8000 & 14.6000 \\
\hline 05 Mar 92 & 17 & 13.0800 & 7.9200 & 14.8400 \\
\hline 05 Mar 92 & 18 & 13.0200 & 7.9100 & 14.8800 \\
\hline 05 Mar 92 & 19 & 13.3500 & 7.8400 & 14.9200 \\
\hline 05 Mar 92 & 20 & 13.9300 & 7.7900 & 14.6900 \\
\hline 05 Mar 92 & 21 & 13.6700 & 7.7700 & 14.8800 \\
\hline $05 \operatorname{Mar} 92$ & 22 & 13.6900 & 7.8100 & 14.4200 \\
\hline 05 Mar 92 & 23 & 14.3600 & 7.8300 & 13.6800 \\
\hline 06 Mar 92 & 0 & 14.3800 & 7.8700 & 13.6600 \\
\hline $06 \operatorname{Mar} 92$ & 1 & 12.9400 & 7.9700 & 14.7500 \\
\hline 06 Mar 92 & 2 & 12.3100 & 8.0200 & 15.2400 \\
\hline 06 Mar 92 & 3 & 12.8600 & 7.9700 & 14.8200 \\
\hline 06 Mar 92 & 4 & 12.5500 & 8.1100 & 15.0300 \\
\hline $06 \operatorname{Mar} 92$ & 5 & 12.1700 & 8.1400 & 15.4000 \\
\hline $06 \operatorname{Mar} 92$ & 6 & 12.2100 & 8.1100 & 15.3700 \\
\hline 06 Mar 92 & 7 & 12.2500 & 8.0800 & 15.3100 \\
\hline 06 Mar 92 & 8 & 13.2100 & 7.9500 & 15.1200 \\
\hline 06 Mar 92 & 9 & 13.3800 & 7.9400 & 15.0900 \\
\hline 06 Mar 92 & 10 & 13.2000 & 7.9800 & 15.2000 \\
\hline 06 Mar 92 & 11 & 13.1200 & 8.0300 & 14.9200 \\
\hline
\end{tabular}




\section{C-53}

Table C.1 (continued)

\begin{tabular}{|c|c|c|c|c|}
\hline Date & Hour & $\begin{array}{c}\text { Temperature } \\
\left({ }^{\circ} \mathrm{C}\right)\end{array}$ & $\mathrm{pH}$ & $\begin{array}{c}\text { Dissolved } \\
\text { oxygen } \\
(\mathrm{mg} / \mathrm{L})\end{array}$ \\
\hline 06 Mar 92 & 12 & 13.3800 & 7.9600 & 14.2900 \\
\hline 06 Mar 92 & 13 & 13.8900 & 8.0000 & 13.9100 \\
\hline 06 Mar 92 & 14 & 13.1500 & 8.0600 & 14.4800 \\
\hline $06 \operatorname{Mar} 92$ & 15 & 12.3200 & 8.1500 & 15.1600 \\
\hline 06 Mar 92 & 16 & 12.5900 & 8.0600 & 15.0000 \\
\hline $12 \operatorname{Mar} 92$ & 0 & & & \\
\hline $13 \operatorname{Mar} 92$ & 17 & 10.4700 & 8.0700 & 16.5500 \\
\hline $13 \operatorname{Mar} 92$ & 18 & 10.7300 & 8.3300 & 17.7800 \\
\hline 13 Mar 92 & 19 & 11.6500 & 8.2500 & 17.6400 \\
\hline $13 \operatorname{Mar} 92$ & 20 & 10.9900 & 8.0900 & 17.4400 \\
\hline 13 Mar 92 & 21 & 9.8300 & 7.9700 & 17.9700 \\
\hline $13 \operatorname{Mar} 92$ & 22 & 9.3800 & 7.9300 & 18.4300 \\
\hline 13 Mar 92 & 23 & 10.4400 & 8.0600 & 17.2400 \\
\hline $14 \operatorname{Mar} 92$ & 0 & 10.6200 & 8.1000 & 16.8100 \\
\hline $14 \operatorname{Mar} 92$ & 1 & 10.6600 & 8.1000 & 16.6600 \\
\hline $14 \operatorname{Mar} 92$ & 2 & 10.5000 & 8.0800 & 16.9200 \\
\hline $14 \operatorname{Mar} 92$ & 3 & 10.3100 & 8.0700 & 17.0500 \\
\hline $14 \operatorname{Mar} 92$ & 4 & 10.2500 & 8.0800 & 17.0000 \\
\hline $14 \operatorname{Mar} 92$ & 5 & 10.2600 & 8.0900 & 16.9600 \\
\hline $14 \operatorname{Mar} 92$ & 6 & 9.9500 & 8.0700 & 17.1500 \\
\hline 14 Mar 92 & 7 & 9.3200 & 8.0200 & 17.6500 \\
\hline $14 \operatorname{Mar} 92$ & 8 & 9.2000 & 8.0200 & 17.6000 \\
\hline $14 \operatorname{Mar} 92$ & 9 & 9.6400 & 8.0600 & 17.0100 \\
\hline $14 \operatorname{Mar} 92$ & 10 & 10.4600 & 8.1300 & 16.9200 \\
\hline $14 \operatorname{Mar} 92$ & 11 & 10.3700 & 8.1000 & 16.6800 \\
\hline $14 \operatorname{Mar} 92$ & 12 & 9.7100 & 8.0700 & 16.9500 \\
\hline 14 Mar 92 & 13 & 9.4300 & 8.0600 & 17.5300 \\
\hline $14 \operatorname{Mar} 92$ & 14 & 10.8400 & 8.1300 & 16.5300 \\
\hline 14 Mar 92 & 15 & 11.0000 & 8.3200 & 16.1100 \\
\hline $14 \operatorname{Mar} 92$ & 16 & 11.2800 & 8.4700 & 15.7200 \\
\hline 14 Mar 92 & 17 & 11.3700 & 8.4600 & 16.3400 \\
\hline 14 Mar 92 & 18 & 10.8400 & 8.8400 & 16.7900 \\
\hline
\end{tabular}




\section{C-54}

Table C.1 (continued)

\begin{tabular}{|c|c|c|c|c|}
\hline Date & Hour & $\begin{array}{c}\text { Temperature } \\
\left({ }^{\circ} \mathrm{C}\right)\end{array}$ & $\mathrm{pH}$ & $\begin{array}{l}\text { Dissolved } \\
\text { oxygen } \\
(\mathrm{mg} / \mathrm{L})\end{array}$ \\
\hline $14 \operatorname{Mar} 92$ & 19 & 10.9400 & 8.5200 & 16.7600 \\
\hline 14 Mar 92 & 20 & 10.5200 & 8.1800 & 16.8600 \\
\hline 14 Mar 92 & 21 & 10.5100 & 8.1000 & 16.9100 \\
\hline 14 Mar 92 & 22 & 10.6700 & 8.1100 & 16.7600 \\
\hline $14 \operatorname{Mar} 92$ & 23 & 10.6600 & 8.1100 & 16.8300 \\
\hline 15 Mar 92 & 0 & 10.6000 & 8.1000 & 17.0100 \\
\hline 15 Mar 92 & 1 & 10.4400 & 8.0800 & 17.1300 \\
\hline 15 Mar 92 & 2 & 10.3800 & 8.0700 & 17.2200 \\
\hline 15 Mar 92 & 3 & 10.3300 & 8.0700 & 17.1500 \\
\hline 15 Mar 92 & 4 & 10.3400 & 8.1000 & 16.9900 \\
\hline 15 Mar 92 & 5 & 10.3000 & 8.0900 & 17.0100 \\
\hline 15 Mar 92 & 6 & 10.2900 & 8.0900 & 16.9900 \\
\hline 15 Mar 92 & 7 & 10.0400 & 8.0400 & 17.1000 \\
\hline $15 \operatorname{Mar} 92$ & 8 & 9.6500 & 7.9700 & 17.2300 \\
\hline 15 Mar 92 & 9 & 9.7500 & 8.0500 & 17.1000 \\
\hline $15 \operatorname{Mar} 92$ & 10 & 10.2700 & 8.0800 & 16.7100 \\
\hline 15 Mar 92 & 11 & 10.2300 & 8.0800 & 17.0600 \\
\hline $15 \operatorname{Mar} 92$ & 12 & 10.2600 & 8.0400 & 17.2800 \\
\hline 15 Mar 92 & 13 & 10.4300 & 8.0600 & 17.1000 \\
\hline 15 Mar 92 & 14 & 10.6500 & 8.3600 & 17.2500 \\
\hline 15 Mar 92 & 15 & 10.4600 & 8.8000 & 17.4400 \\
\hline $15 \operatorname{Mar} 92$ & 16 & 10.7100 & 8.4300 & 17.5100 \\
\hline $20 \operatorname{Mar} 92$ & 0 & & & \\
\hline 21 Mar 92 & 12 & 11.4500 & 8.1800 & 16.0300 \\
\hline 21 Mar 92 & 13 & 11.2500 & 7.7300 & 15.2200 \\
\hline 21 Mar 92 & 14 & 12.0300 & 7.9100 & 15.0800 \\
\hline 21 Mar 92 & 15 & 12.7000 & 8.6600 & 15.0000 \\
\hline 21 Mar 92 & 16 & 13.1400 & 8.6000 & 14.7200 \\
\hline 21 Mar 92 & 17 & 13.3400 & 8.8400 & 14.6500 \\
\hline 21 Mar 92 & 18 & 13.4900 & 8.7000 & 14.4400 \\
\hline 21 Mar 92 & 19 & 13.6600 & 8.1800 & 14.3500 \\
\hline 21 Mar 92 & 20 & 13.5300 & 8.3500 & 14.9700 \\
\hline
\end{tabular}


Table C.1 (continued)

\begin{tabular}{|c|c|c|c|c|}
\hline Date & Hour & $\begin{array}{l}\text { Temperature } \\
\left({ }^{\circ} \mathrm{C}\right)\end{array}$ & $\mathrm{pH}$ & $\begin{array}{l}\text { Dissolved } \\
\text { axygen } \\
(\mathrm{mg} / \mathrm{L})\end{array}$ \\
\hline 21 Mar 92 & 21 & 13.3800 & 8.3700 & 15.3400 \\
\hline $21 \operatorname{Mar} 92$ & 22 & 13.3800 & 8.3000 & 15.1100 \\
\hline 21 Mar 92 & 23 & 13.2200 & 8.3800 & 15.5300 \\
\hline $22 \operatorname{Mar} 92$ & 0 & 13.0700 & 8.3500 & 15.5100 \\
\hline $22 \operatorname{Mar} 92$ & 1 & 12.9500 & 8.2500 & 15.2600 \\
\hline 22 Mar 92 & 2 & 12.8700 & 8.0500 & 14.5200 \\
\hline $22 \operatorname{Mar} 92$ & 3 & 12.6200 & 8.0100 & 14.4800 \\
\hline $22 \operatorname{Mar} 92$ & 4 & 12.3700 & 8.0100 & 14.5500 \\
\hline $22 \operatorname{Mar} 92$ & 5 & 12.0300 & 7.9900 & 14.7500 \\
\hline $22 \operatorname{Mar} 92$ & 6 & 11.7300 & 8.0000 & 14.8700 \\
\hline $22 \operatorname{Mar} 92$ & 7 & 11.5600 & 8.0400 & 14.9900 \\
\hline $22 \operatorname{Mar} 92$ & 8 & 11.3600 & 8.0200 & 15.0900 \\
\hline $22 \operatorname{Mar} 92$ & 9 & 11.6600 & 8.2000 & 15.5300 \\
\hline $22 \operatorname{Mar} 92$ & 10 & 12.0200 & 8.3100 & 15.7800 \\
\hline $22 \operatorname{Mar} 92$ & 11 & 11.2400 & 8.1900 & 15.0800 \\
\hline 22 Mar 92 & 12 & 11.0500 & 8.3200 & 14.9500 \\
\hline $22 \operatorname{Mar} 92$ & 13 & 11.1000 & 7.9900 & 15.0100 \\
\hline 22 Mar 92 & 14 & 11.1400 & 8.4300 & 15.0000 \\
\hline 22. Mar 92 & 15 & 11.3100 & 8.7800 & 15.1200 \\
\hline $22 \operatorname{Mar} 92$ & 16 & 11.3000 & 8.5200 & 15.1000 \\
\hline $22 \operatorname{Mar} 92$ & 17 & 11.3900 & 8.4000 & 15.1800 \\
\hline $22 \operatorname{Mar} 92$ & 18 & 11.3200 & 8.0900 & 15.1700 \\
\hline $26 \operatorname{Mar} 92$ & $\mathbf{0}$ & & & \\
\hline 27 Mar 92 & 15 & 11.9400 & 8.0200 & 10.9200 \\
\hline 27 Mar 92 & 16 & 12.3100 & 7.8600 & 14.3000 \\
\hline 27 Mar 92 & 17 & 12.6300 & 7.8700 & 14.2500 \\
\hline 27 Mar 92 & 18 & 12.7600 & 8.0600 & 15.0800 \\
\hline $27 \operatorname{Mar} 92$ & 19 & 12.8700 & 8.0800 & 15.2900 \\
\hline 27 Mar 92 & 20 & 12.8300 & 8.1200 & 15.5300 \\
\hline 27 Mar 92 & 21 & 12.8200 & 8.1300 & 15.6700 \\
\hline 27 Mar 92 & 22 & 12.7500 & 8.1300 & 15.5900 \\
\hline 27 Mar 92 & 23 & 12.5800 & 8.1400 & 15.7800 \\
\hline
\end{tabular}




\section{C-56}

Table C.1 (continued)

\begin{tabular}{|c|c|c|c|c|}
\hline Date & Hour & $\begin{array}{c}\text { Temperature } \\
\left({ }^{\circ} \mathrm{C}\right)\end{array}$ & $\mathrm{pH}$ & $\begin{array}{c}\text { Dissolved } \\
\text { oxygen } \\
(\mathrm{mg} / \mathrm{L})\end{array}$ \\
\hline $28 \operatorname{Mar} 92$ & 0 & 12.5500 & 8.1200 & 15.6100 \\
\hline 28 Mar 92 & 1 & 12.7000 & 8.0700 & 15.4100 \\
\hline $28 \operatorname{Mar} 92$ & 2 & 12.3700 & 8.1000 & 15.9300 \\
\hline $28 \operatorname{Mar} 92$ & 3 & 12.4000 & 8.0200 & 15.4100 \\
\hline 28 Mar 92 & 4 & 12.3000 & 8.0600 & 15.1500 \\
\hline $28 \operatorname{Mar} 92$ & 5 & 12.1100 & 7.9900 & 14.5200 \\
\hline $28 \operatorname{Mar} 92$ & 6 & 11.8700 & 7.9100 & 14.4900 \\
\hline $28 \operatorname{Mar} 92$ & 7 & 11.6700 & 7.8900 & 14.5800 \\
\hline $28 \operatorname{Mar} 92$ & 8 & 11.5200 & 7.9000 & 14.5500 \\
\hline $28 \operatorname{Mar} 92$ & 9 & 11.4500 & 7.9200 & 14.6900 \\
\hline $28 \operatorname{Mar} 92$ & 10 & 11.5100 & 7.8300 & 14.8800 \\
\hline 28 Mar 92 & 11 & 11.3700 & 7.9200 & 14.7100 \\
\hline 28 Mar 92 & 12 & 11.4000 & 7.6800 & 14.6400 \\
\hline 28 Mar 92 & 13 & 11.6900 & 7.7200 & 14.6900 \\
\hline $28 \operatorname{Mar} 92$ & 14 & 12.1400 & 7.7500 & 14.5800 \\
\hline $28 \operatorname{Mar} 92$ & 15 & 12.8100 & 7.8700 & 14.3500 \\
\hline $28 \operatorname{Mar} 92$ & 16 & 13.4200 & 8.0500 & 14.2300 \\
\hline 28 Mar 92 & 17 & 13.5500 & 8.1100 & 14.6800 \\
\hline $28 \operatorname{Mar} 92$ & 18 & 13.2700 & 8.0600 & 15.1700 \\
\hline 28 Mar 92 & 19 & 13.4400 & 8.0000 & 14.8600 \\
\hline $28 \operatorname{Mar} 92$ & 20 & 13.1500 & 8.0500 & 15.3300 \\
\hline 28 Mar 92 & 21 & 13.2600 & 8.0800 & 15.3400 \\
\hline 28 Mar 92 & 22 & 13.3400 & 8.0300 & 15.2400 \\
\hline $28 \operatorname{Mar} 92$ & 23 & 13.2800 & 8.0700 & 15.3500 \\
\hline $29 \operatorname{Mar} 92$ & 0 & 13.2600 & 8.1000 & 15.1900 \\
\hline $29 \operatorname{Mar} 92$ & 1 & 13.2800 & 8.1400 & 14.7500 \\
\hline 29 Mar 92 & 2 & 13.0600 & 8.1000 & 15.0400 \\
\hline 29 Mar 92 & 3 & 12.8800 & 8.0600 & 15.5800 \\
\hline $29 \operatorname{Mar} 92$ & 4 & 12.8200 & 8.1100 & 15.2100 \\
\hline 29 Mar 92 & 5 & 12.7700 & 8.0600 & 14.5900 \\
\hline $29 \operatorname{Mar} 92$ & 6 & 12.5600 & 7.8400 & 14.26 .30 \\
\hline $29 \operatorname{Mar} 92$ & 7 & 12.3200 & 7.8000 & 14.2400 \\
\hline
\end{tabular}




\section{C-57}

Table C.1 (continued)

\begin{tabular}{|c|c|c|c|c|}
\hline Date & Hour & $\begin{array}{c}\text { Temperature } \\
\left({ }^{\circ} \mathrm{C}\right)\end{array}$ & $\mathrm{pH}$ & $\begin{array}{c}\text { Dissolved } \\
\text { axygen } \\
(\mathrm{mg} / \mathrm{L}) \\
\end{array}$ \\
\hline $29 \operatorname{Mar} 92$ & 8 & 12.1200 & 7.8800 & 14.3300 \\
\hline $29 \operatorname{Mar} 92$ & 9 & 12.0800 & 7.9500 & 15.2900 \\
\hline $29 \operatorname{Mar} 92$ & 10 & 11.9100 & 7.8100 & 14.6300 \\
\hline 29 Mar 92 & 11 & 11.8000 & 7.8300 & 14.3100 \\
\hline 29 Mar 92 & 12 & 11.7900 & 7.7100 & 14.3200 \\
\hline $29 \operatorname{Mar} 92$ & 13 & 11.8200 & 7.7500 & 14.5100 \\
\hline $29 \operatorname{Mar} 92$ & 14 & 11.7800 & 7.7300 & 14.2500 \\
\hline $29 \operatorname{Mar} 92$ & 15 & 11.8300 & 7.7600 & 14.3100 \\
\hline $29 \operatorname{Mar} 92$ & 16 & 11.8900 & 7.7500 & 14.3800 \\
\hline $29 \operatorname{Mar} 92$ & 17 & 12.0000 & 7.7700 & 14.4000 \\
\hline $29 \operatorname{Mar} 92$ & 18 & 12.0900 & 7.7800 & 14.3100 \\
\hline $29 \operatorname{Mar} 92$ & 19 & 12.1300 & 7.8000 & 14.3400 \\
\hline 30 Mar 92 & 0 & & & \\
\hline 31 Mar 92 & 14 & 12.5100 & 7.8500 & 17.4300 \\
\hline 31 Mar 92 & 15 & 12.7000 & 7.8400 & 17.4800 \\
\hline 31 Mar 92 & 16 & 13.3400 & 8.0200 & 16.6300 \\
\hline 31 Mar 92 & 17 & 13.2600 & 8.0400 & 16.9600 \\
\hline 31 Mar 92 & 18 & 13.4200 & 8.1200 & 17.1600 \\
\hline 31 Mar 92 & 19 & 13.0100 & 8.1400 & 17.3500 \\
\hline 31 Mar 92 & 20 & 12.5500 & 8.0000 & 17.9000 \\
\hline 31 Mar 92 & 21 & 12.4400 & 8.0000 & 18.0700 \\
\hline 31 Mar 92 & 22 & 12.3800 & 8.0500 & 18.2600 \\
\hline $31 \operatorname{Mar} 92$ & 23 & 12.3300 & 8.1400 & 18.2900 \\
\hline 01 Apr 92 & 0 & 12.3000 & 8.1000 & 18.1600 \\
\hline 01 Apr 92 & 1 & 12.2300 & 8.1200 & 18.1300 \\
\hline 01 Apr 92 & 2 & 12.1800 & 8.1500 & 18.0900 \\
\hline 01 Apr 92 & 3 & 12.1500 & 8.1700 & 18.0800 \\
\hline 01 Apr 92 & 4 & 12.1100 & 8.1900 & 18.0000 \\
\hline 01 Apr 92 & 5 & 12.0700 & 8.2100 & 17.8700 \\
\hline 01 Apr 92 & 6 & 12.0100 & 8.2500 & 17.9300 \\
\hline 01 Apr 92 & 7 & 11.9400 & 8.2500 & 18.0900 \\
\hline 01 Apr 92 & 8 & 11.9000 & 8.2000 & 17.7400 \\
\hline
\end{tabular}




\section{C-58}

Table C.1 (continued)

\begin{tabular}{|c|c|c|c|c|}
\hline Date & Hour & $\begin{array}{l}\text { Temperature } \\
\left({ }^{\circ} \mathrm{C}\right)\end{array}$ & $\mathrm{pH}$ & $\begin{array}{l}\text { Dissolved } \\
\text { axygen } \\
\text { (mg/L) }\end{array}$ \\
\hline 01 Apr 92 & 9 & 11.9900 & 8.2000 & 17.5000 \\
\hline $01 \mathrm{Apr} 92$ & 10 & 11.8200 & 8.0800 & 16.5300 \\
\hline 01 Apr 92 & 11 & 11.3000 & 7.8300 & 15.3300 \\
\hline 01 Apr 92 & 12 & 11.2700 & 7.8400 & 15.7000 \\
\hline 01 Apr 92 & 13 & 11.2700 & 8.0300 & 16.1600 \\
\hline 01 Apr 92 & 14 & 11.4500 & 8.0100 & 15.8700 \\
\hline 01 Apr 92 & 15 & 11.7600 & 8.0600 & 16.5200 \\
\hline $01 \mathrm{Apr} 92$ & 16 & 11.7800 & 8.0300 & 16.5100 \\
\hline 01 Apr 92 & 17 & 11.7500 & 7.9600 & 16.2900 \\
\hline 01 Apr 92 & 18 & 11.7100 & 7.9400 & 16.1800 \\
\hline 01 Apr 92 & 19 & 11.6500 & 7.9300 & 16.1700 \\
\hline 01 Apr 92 & 20 & 11.6100 & 7.8900 & 16.0800 \\
\hline 01 Apr 92 & 21 & 11.5700 & 7.9000 & 16.1100 \\
\hline 01 Apr 92 & 22 & 11.5200 & 7.9700 & 16.3200 \\
\hline 01 Apr 92 & 23 & 11.3700 & 7.9300 & 16.3600 \\
\hline 02 Apr 92 & 0 & 11.2500 & 7.9400 & 16.3800 \\
\hline 02 Apr 92 & 1 & 11.1000 & 7.9500 & 16.3700 \\
\hline 02 Apr 92 & 2 & 10.9400 & 7.9400 & 16.3600 \\
\hline 02 Apr 92 & 3 & 10.7900 & 7.9300 & 16.3600 \\
\hline 02 Apr 92 & 4 & 10.6200 & 7.9100 & 16.3700 \\
\hline 02 Apr 92 & 5 & 10.4900 & 7.9100 & 16.3800 \\
\hline 02 Apr 92 & 6 & 10.4500 & 7.9300 & 16.4300 \\
\hline 02 Apr 92 & 7 & 10.9000 & 8.0800 & 16.6800 \\
\hline 02 Apr 92 & 8 & 11.0600 & 8.1700 & 17.3000 \\
\hline 02 Apr 92 & 9 & 11.1800 & 8.2000 & 17.3200 \\
\hline 02 Apr 92 & 10 & 11.2900 & 8.2400 & 17.3800 \\
\hline 02 Apr 92 & 11 & 10.9800 & 8.2100 & 17.4200 \\
\hline 02 Apr 92 & 12 & 11.3900 & 8.2800 & 17.8300 \\
\hline 02 Apr 92 & 13 & 11.6400 & 8.3500 & 17.9600 \\
\hline 02 Apr 92 & 14 & 11.9100 & 8.4200 & 18.1400 \\
\hline 02 Apr 92 & 15 & 11.9600 & 8.4400 & 18.0600 \\
\hline 02 Apr 92 & 16 & 11.9400 & 8.4600 & 18.3100 \\
\hline
\end{tabular}


C-59

Table C.1 (continued)

\begin{tabular}{|c|c|c|c|c|}
\hline Date & Hour & $\begin{array}{c}\text { Temperature } \\
\left({ }^{\circ} \mathrm{C}\right)\end{array}$ & $\mathrm{pH}$ & $\begin{array}{c}\text { Dissolved } \\
\text { oxygen } \\
\text { (mg/L) }\end{array}$ \\
\hline 02 Apr 92 & 17 & 11.9700 & 8.4800 & 18.3800 \\
\hline 02 Apr 92 & 18 & 11.9200 & 8.4900 & 18.4400 \\
\hline 02 Apr 92 & 19 & 11.7600 & 8.4700 & 18.3900 \\
\hline 02 Apr 92 & 20 & 11.6900 & 8.4300 & 18.3100 \\
\hline 02 Apr 92 & 21 & 11.4700 & 8.3500 & 17.8000 \\
\hline 02 or 92 & 22 & 10.9000 & 8.2300 & 17.3100 \\
\hline 02 Apr 92 & 23 & 10.1600 & 8.0000 & 17.0100 \\
\hline 03 Apr 92 & 0 & 10.0500 & 7.9400 & 16.9800 \\
\hline 03 Apr 92 & 1 & 9.9800 & 7.9200 & 16.7800 \\
\hline 03 Apr 92 & 2 & 9.9100 & 7.9100 & 16.8600 \\
\hline 03 Apr 92 & 3 & 9.9600 & 7.9800 & 17.1600 \\
\hline 03 Apr 92 & 4 & 10.0500 & 8.0600 & 17.3600 \\
\hline 03 Apr 92 & 5 & 10.2900 & 8.1900 & 17.8500 \\
\hline 03 Apr 92 & 6 & 10.8300 & 8.2800 & 17.8200 \\
\hline 03 Apr 92 & 7 & 10.8700 & 8.2800 & 17.9300 \\
\hline 03 Apr 92 & 8 & 10.5300 & 8.2300 & 17.7200 \\
\hline 03 Apr 92 & 9 & 10.4300 & 8.2300 & 17.5500 \\
\hline 03 Apr 92 & 10 & 10.5200 & 8.2400 & 17.6400 \\
\hline 03 Apr 92 & 11 & 10.2800 & 8.2100 & 17.8200 \\
\hline 03 Apr 92 & 12 & 10.2200 & 8.1500 & 17.5400 \\
\hline 03 Apr 92 & 13 & 9.6500 & 8.0600 & 17.7600 \\
\hline 03 Apr 92 & 14 & 9.9500 & 8.0600 & 17.4600 \\
\hline 03 Apr 92 & 15 & 11.2400 & 8.2500 & 17.6600 \\
\hline 03 Apr 92 & 16 & 11.4200 & 8.2600 & 17.2900 \\
\hline 03 Apr 92 & 17 & 11.4600 & 8.2600 & 17.3500 \\
\hline 03 Apr 92 & 18 & 11.3600 & 8.2100 & 17.2600 \\
\hline 04 Apr 92 & 12 & 11.1800 & 8.1100 & 17.5600 \\
\hline 04 Apr 92 & 13 & 10.9900 & 8.0900 & 17.1500 \\
\hline 04 Apr 92 & 14 & 11.3000 & 8.1300 & 16.9800 \\
\hline 04 Apr 92 & 15 & 11.5300 & 8.1600 & 16.7100 \\
\hline 04 Apr 92 & 16 & 11.7200 & 8.2200 & 16.8300 \\
\hline 04 Apr 92 & 17 & 11.7500 & 8.1700 & 16.5300 \\
\hline
\end{tabular}


C-60

Table C.1 (continued)

\begin{tabular}{|c|c|c|c|c|}
\hline Date & Hour & $\begin{array}{l}\text { Temperature } \\
\left({ }^{\circ} \mathrm{C}\right)\end{array}$ & $\mathrm{pH}$ & $\begin{array}{c}\text { Dissolved } \\
\text { axygen } \\
(\mathrm{mg} / \mathrm{L})\end{array}$ \\
\hline 04 Apr 92 & 18 & 11.8400 & 8.1300 & 16.2900 \\
\hline 04 Apr 92 & 19 & 12.0100 & 8.1200 & 16.1300 \\
\hline 04 Apr 92 & 20 & 12.2000 & 8.1300 & 16.0600 \\
\hline 04 Apr 92 & 21 & 12.3000 & 8.1400 & 15.9900 \\
\hline 04 Apr 92 & 22 & 12.3100 & 8.1600 & 16.0000 \\
\hline 04 Apr 92 & 23 & 12.2600 & 8.2200 & 16.1000 \\
\hline 05 Apr 92 & 0 & 12.1600 & 8.2600 & 16.3100 \\
\hline 05 Apr 92 & 1 & 12.0600 & 8.2500 & 16.1800 \\
\hline 05 Apr 92 & 2 & 11.9800 & 8.2600 & 16.2200 \\
\hline 05 Apr 92 & 3 & 11.8900 & 8.3500 & 16.6900 \\
\hline 05 Apr 92 & 4 & 11.8400 & 8.3700 & 16.7200 \\
\hline 05 Apr 92 & 5 & 11.7600 & 8.3800 & 16.7500 \\
\hline 05 Apr 92 & 6 & 11.6800 & 8.3900 & 16.7100 \\
\hline 05 Apr 92 & 7 & 11.6300 & 8.3900 & 16.9400 \\
\hline 05 Apr 92 & 8 & 11.5900 & 8.3900 & 17.3000 \\
\hline 05 Apr 92 & 9 & 11.6400 & 8.3700 & 17.4700 \\
\hline 05 Apr 92 & 10 & 11.6900 & 8.4200 & 17.4700 \\
\hline 05 Apr 92 & 11 & 11.8400 & 8.7400 & 17.2600 \\
\hline 05 Apr 92 & 12 & 12.1500 & 8.9600 & 16.9900 \\
\hline 05 Apr 92 & 13 & 12.3200 & 8.6900 & $17.350 x$ \\
\hline 05 Apr 92 & 14 & 12.5100 & 8.6200 & 17.3900 \\
\hline 05 Apr 92 & 15 & 12.6700 & 8.6100 & 17.5900 \\
\hline 05 Apr 92 & 16 & 12.8600 & 8.5700 & 17.5400 \\
\hline 05 Apr 92 & 17 & 13.0500 & 8.6500 & 17.4500 \\
\hline 05 Apr 92 & 18 & 13.2500 & 8.6100 & 17.2000 \\
\hline 05 Apr 92 & 19 & 13.3500 & 8.5400 & 16.7500 \\
\hline 06 Apr 92 & 9 & 12.2000 & 8.4500 & 10.3900 \\
\hline 06 Apr 92 & 10 & 12.2600 & 8.5400 & 10.4000 \\
\hline 06 Apr 92 & 11 & 12.3200 & 8.4900 & 10.2500 \\
\hline 06 Apr 92 & 12 & 12.3900 & 8.5200 & 11.4100 \\
\hline 06 Apr 92 & 13 & 12.4200 & 8.5700 & 16.9300 \\
\hline 06 Apr 92 & 14 & 12.5500 & 8.5400 & 18.0300 \\
\hline
\end{tabular}


Table C.1 (continued)

\begin{tabular}{|c|c|c|c|c|}
\hline Date & Hour & $\begin{array}{c}\text { Temperature } \\
\left({ }^{\circ} \mathrm{C}\right)\end{array}$ & $\mathrm{pH}$ & $\begin{array}{c}\text { Dissolved } \\
\text { axygen } \\
(\mathrm{mg} / \mathrm{L})\end{array}$ \\
\hline 06 Apr 92 & 15 & 12.5800 & 8.5500 & 17.7500 \\
\hline 06 Apr 92 & 16 & 12.5800 & 8.5700 & 18.0000 \\
\hline 06 Apr 92 & 17 & 12.6400 & 8.4800 & 17.2600 \\
\hline 06 Apr 92 & 18 & 12.1000 & 8.0600 & 15.0800 \\
\hline 06 Apr 92 & 19 & 12.4100 & 8.1300 & 15.3000 \\
\hline 06 Apr 92 & 20 & 12.6900 & 8.2900 & 15.8200 \\
\hline 06 Apr 92 & 21 & 12.8700 & 8.3600 & 16.1100 \\
\hline 06 Apr 92 & 22 & 13.0700 & 8.3600 & 16.1100 \\
\hline 06 Apr 92 & 23 & 13.1300 & 8.2900 & 15.7700 \\
\hline 07 Apr 92 & 0 & 13.1000 & 8.2300 & 15.4200 \\
\hline 07 Apr 92 & 1 & 13.0400 & 8.1800 & 15.1700 \\
\hline 07 Apr 92 & 2 & 13.0000 & 8.1600 & 15.0300 \\
\hline 07 Apr 92 & 3 & 12.9600 & 8.1400 & 14.9100 \\
\hline 07 Apr 92 & 4 & 12.9000 & 8.1500 & 15.1000 \\
\hline 07 Apr 92 & 5 & 12.8000 & 8.1800 & 15.3200 \\
\hline 07 Apr 92 & 6 & 12.7500 & 8.1500 & 14.9900 \\
\hline 07 Apr 92 & 7 & 12.8800 & 8.1200 & 14.6600 \\
\hline 07 Apr 92 & 8 & 12.8700 & 8.1000 & 13.6400 \\
\hline 07 Apr 92 & 9 & 12.8700 & 8.0900 & 14.5400 \\
\hline 07 Apr 92 & 10 & 12.7800 & 8.1100 & 14.5700 \\
\hline 07 Apr 92 & 11 & 12.8500 & 8.1200 & 14.4700 \\
\hline 07 Apr 92 & 12 & 12.8500 & 8.3300 & 14.5300 \\
\hline 07 Apr 92 & 13 & 12.8400 & 8.2000 & 14.4900 \\
\hline 07 Apr 92 & 14 & 12.8500 & 8.1300 & 14.3900 \\
\hline 07 Apr 92 & 15 & 12.9100 & 8.1300 & 14.4300 \\
\hline 07 Apr 92 & 16 & 12.9600 & 8.1300 & 14.4700 \\
\hline 07 Apr 92 & 17 & 12.7800 & 8.3600 & 15.8600 \\
\hline 07 Apr 92 & 18 & 12.6400 & 8.3600 & 16.5400 \\
\hline 07 Apr 92 & 19 & 12.6300 & 8.3500 & 16.4000 \\
\hline 07 Apr 92 & 20 & 12.5400 & 8.4300 & 16.7600 \\
\hline 07 Apr 92 & 21 & 12.9400 & 8.4800 & 15.6800 \\
\hline 07 Apr 92 & 22 & 13.1100 & 8.2200 & 15.0200 \\
\hline
\end{tabular}




\section{C-62}

Table C.1 (continued)

\begin{tabular}{|c|c|c|c|c|}
\hline Date & Hcur & $\begin{array}{c}\text { Temperature } \\
\left({ }^{\circ} \mathrm{C}\right)\end{array}$ & $\mathrm{pH}$ & $\begin{array}{c}\text { Dissolved } \\
\text { oxygen } \\
(\mathrm{mg} / \mathrm{L})\end{array}$ \\
\hline 07 Apr 92 & 23 & 13.1800 & 8.1900 & 14.8400 \\
\hline 08 Apr 92 & 0 & 13.1700 & 8.1700 & 14.8700 \\
\hline 08 Apr 92 & 1 & 13.0900 & 8.1900 & 15.0400 \\
\hline 08 Apr 92 & 2 & 12.9400 & 8.2500 & 15.3300 \\
\hline 08 Apr 92 & 3 & 12.8600 & 8.2600 & 15.4800 \\
\hline 08 Apr 92 & 4 & 12.8400 & 8.2300 & 15.3200 \\
\hline 08 Apr 92 & 5 & 12.8000 & 8.2300 & 15.4200 \\
\hline 08 Apr 92 & 6 & 12.5300 & 8.3000 & 16.0500 \\
\hline 08 Apr 92 & 7 & 12.7800 & 8.3000 & 15.5100 \\
\hline 08 Apr 92 & 8 & 12.2900 & 8.3600 & 16.2800 \\
\hline 08 Apr 92 & 9 & 12.2600 & 8.3800 & 16.5700 \\
\hline 08 Apr 92 & 10 & 12.5800 & 8.3100 & 15.5000 \\
\hline 08 Apr 92 & 11 & 12.9400 & 8.0700 & 14.2300 \\
\hline 08 Apr 92 & 12 & 13.0600 & 8.0500 & 14.1400 \\
\hline 08 Apr 92 & 13 & 13.3000 & 8.0400 & 12.3000 \\
\hline 08 Apr 92 & 14 & 13.5200 & 8.0900 & 13.6900 \\
\hline 08 Apr 92 & 15 & 13.8100 & 8.1100 & 14.0500 \\
\hline 08 Apr 92 & 16 & 14.1100 & 8.2000 & 13.9800 \\
\hline 08 Apr 92 & 17 & 14.1400 & 8.1900 & 14.1800 \\
\hline 08 Apr 92 & 18 & 13.6400 & 8.4500 & 16.0800 \\
\hline 08 Apr 92 & 19 & 13.3300 & 8.5500 & 17.2900 \\
\hline 08 Apr 92 & 20 & 13.2800 & 8.5700 & 17.7900 \\
\hline 08 Apr 92 & 21 & 13.0000 & 8.5000 & 17.0800 \\
\hline 08 Apr 92 & 22 & 12.6200 & 8.5200 & 17.0900 \\
\hline 08 Apr 92 & 23 & 12.5700 & 8.5300 & 16.9900 \\
\hline 09 Apr 92 & 0 & 12.5400 & 8.5300 & 16.9600 \\
\hline 09 Apr 92 & 1 & 12.4600 & 8.5300 & 17.0300 \\
\hline 09 Apr 92 & 2 & 12.4500 & 8.5200 & 17.0200 \\
\hline 09 Apr 92 & 3 & 12.5000 & 8.5000 & 16.8100 \\
\hline 09 Apr 92 & 4 & 12.4900 & 8.5100 & 16.6800 \\
\hline 09 Apr 92 & 5 & 12.4500 & 8.5000 & 16.7000 \\
\hline 09 Apr 92 & 6 & 12.3100 & 8.4900 & 17.1300 \\
\hline
\end{tabular}


Table C.1 (continued)

\begin{tabular}{|c|c|c|c|c|}
\hline Date & Hour & $\begin{array}{c}\text { Temperature } \\
\left({ }^{\circ} \mathrm{C}\right)\end{array}$ & $\mathrm{pH}$ & $\begin{array}{c}\text { Dissolved } \\
\text { axygen } \\
(\mathrm{mg} / \mathrm{L})\end{array}$ \\
\hline 09 Apr 92 & 7 & 12.1900 & 8.4700 & 17.1900 \\
\hline 09 Apr 92 & 8 & 12.2800 & 8.5100 & 17.2900 \\
\hline 09 Apr 92 & 9 & 12.4100 & 8.5400 & 17.4300 \\
\hline 09 Apr 92 & 10 & 12.6800 & 8.5700 & 16.9200 \\
\hline 09 Apr 92 & 11 & 12.8700 & 8.5900 & 17.2200 \\
\hline 09 Apr 92 & 12 & 13.9400 & 8.5800 & 16.6200 \\
\hline 09 Apr 92 & 13 & 14.9200 & 8.7500 & 14.3700 \\
\hline 09 Apr 92 & 14 & 15.0800 & 8.4500 & 13.2900 \\
\hline $09 \mathrm{Apr} 92$ & 15 & 3.0700 & 8.3700 & 13.6200 \\
\hline 09 Apr 92 & 16 & 15.8300 & 8.6100 & 14.5600 \\
\hline 09 Apr 92 & 17 & 16.0400 & 8.7000 & 14.8000 \\
\hline 09 Apr 92 & 18 & 15.4700 & 8.3400 & 14.0800 \\
\hline 09 Apr 92 & 19 & 15.5500 & 8.3700 & 14.2200 \\
\hline 09 Apr 92 & 20 & 15.5500 & 8.4500 & 15.5300 \\
\hline 09 Apr 92 & 21 & 16.1500 & 8.4200 & 14.4100 \\
\hline 09 Apr 92 & 22 & 16.8200 & 8.4400 & 13.9000 \\
\hline 09 Apr 92 & 23 & 16.3300 & 8.4600 & 14.4600 \\
\hline $10 \mathrm{Apr} 92$ & 0 & 16.2100 & 8.4200 & 14.2300 \\
\hline 10 Apr 92 & 1 & 16.0400 & 8.3900 & 14.0900 \\
\hline 10 Apr 92 & 2 & 15.8100 & 8.3500 & 14.1400 \\
\hline 10 Apr 92 & 3 & 15.6200 & 8.3300 & 14.0600 \\
\hline 10 Apr 92 & 4 & 15.3400 & 8.3200 & 14.0600 \\
\hline 10 Apr 92 & 5 & 15.2100 & 8.3100 & 14.1200 \\
\hline $10 \mathrm{Apr} 92$ & 6 & 15.2400 & 8.2600 & 13.8500 \\
\hline 10 Apr 92 & 7 & 15.3000 & 8.3500 & 13.5500 \\
\hline 10 Apr 92 & 8 & 15.3000 & 8.4900 & 13.6800 \\
\hline 10 Apr 92 & 9 & 15.3400 & 8.3700 & 13.6400 \\
\hline 10 Apr 92 & 10 & 15.3500 & 8.6400 & 13.5300 \\
\hline 10 Apr 92 & 11 & 15.7500 & 8.4000 & 13.1500 \\
\hline 10 Apr 92 & 12 & 15.6200 & 8.3700 & 13.5900 \\
\hline 10 Apr 92 & 13 & 16.0400 & 8.4500 & 13.9600 \\
\hline 10 Apr 92 & 14 & 15.3100 & 8.4500 & 15.1100 \\
\hline
\end{tabular}




\section{C-64}

Table C.1 (continued)

\begin{tabular}{|c|c|c|c|c|}
\hline Date & Hour & $\begin{array}{c}\text { Temperature } \\
\left({ }^{\circ} \mathrm{C}\right)\end{array}$ & $\mathrm{pH}$ & $\begin{array}{c}\text { Dissolved } \\
\text { oxygen } \\
(m g / L)\end{array}$ \\
\hline 10 Apr 92 & 15 & 14.6500 & 8.4700 & 15.4000 \\
\hline 10 Apr 92 & 16 & 14.7700 & 8.4900 & 15.6600 \\
\hline 10 Apr 92 & 17 & 14.8700 & 8.5200 & 15.8200 \\
\hline 10 Apr 92 & 18 & 15.3900 & 8.5300 & 15.3500 \\
\hline 10 Apr 92 & 19 & 13.9200 & 8.5800 & 15.9400 \\
\hline
\end{tabular}


Appendix D

WATER GRAB SAMPLE ANALYTICAL RESULTS FOR MONITORING OF SPECIFIC CONSTRUCTION ACTIVITIES 
D-3

Table D.1. Water grab sample analytical results for monitoring of specific construction activities

\begin{tabular}{|c|c|c|c|c|c|c|c|c|}
\hline Date & $\begin{array}{l}{ }^{15} \mathrm{Cs} \\
\text { result }\end{array}$ & $\begin{array}{l}\text { Rad. } \\
\text { error }\end{array}$ & Units & $\begin{array}{c}\text { TSP } \\
(\mathrm{mg} / \mathrm{L})\end{array}$ & $\begin{array}{l}\text { Construction } \\
\text { activity }\end{array}$ & $\begin{array}{l}{ }^{137} \mathrm{Cs} \\
\text { result }\end{array}$ & $\begin{array}{l}\text { Rad. } \\
\text { error }\end{array}$ & Units \\
\hline 20 Aug 91 & 0.03 & 0.57 & $\mathrm{~Bq} / \mathrm{L}$ & & Sheet Pile & 0.8 & 15.4 & pCi/L \\
\hline 20 Aug 91 & -0.05 & 0.49 & $B q / L$ & & Sheet pile & -1.4 & 13.2 & $\mathrm{pCi} / \mathrm{L}$ \\
\hline 21 Aug 91 & 0.21 & 0.47 & $\mathrm{~Bq} / \mathrm{L}$ & & Sheet pile & 5.7 & 12.7 & $\mathrm{pCi} / \mathrm{L}$ \\
\hline 21 Aug 91 & 0.29 & 0.67 & $\mathrm{~Bq} / \mathrm{L}$ & & Sheet pile & 7.8 & 18.1 & pCin \\
\hline 11 Feb 92 & 12.90 & 1.50 & $\mathrm{pCi} / \mathrm{L}$ & 10.0 & Jet grout & 1290.0 & 150.0 & $\mathrm{pC} / \mathrm{g}$ \\
\hline 11 Feb 92 & 8.20 & 1.30 & $\mathrm{pCi} / \mathrm{L}$ & 7.0 & Jet grout & 1171.4 & 185.7 & $\mathrm{pCi} / \mathrm{g}$ \\
\hline 13 Feb 92 & 6.60 & 1.00 & $\mathrm{pCi} / \mathrm{L}$ & 8.4 & Jet grout & 785.7 & 119.0 & $\mathrm{pCi} / \mathrm{s}$ \\
\hline 13 Feb 92 & 9.10 & 1.00 & $\mathrm{pCi} / \mathrm{L}$ & 8.5 & Jet grout & 1070.6 & 117.6 & $\mathrm{pCV} / \mathrm{g}$ \\
\hline 13 Feb 92 & 18.70 & 1.40 & $\mathrm{pCi} / \mathrm{L}$ & 22.6 & Jet grout & 827.4 & 61.9 & $\mathrm{pC} / \mathrm{g}$ \\
\hline 13 Feb 92 & 12.8 & 1.3 & $\mathrm{pCi} / \mathrm{L}$ & 18.9 & Jet grout & 677.2 & 68.8 & $\mathrm{PCi} / \mathrm{g}$ \\
\hline 14 Feb 92 & 4.10 & 1.10 & $\mathrm{pCi} / \mathrm{L}$ & 7.0 & Jet grout & 585.7 & 157.1 & $\mathrm{pCV} / \mathrm{g}$ \\
\hline 14 Feb 92 & 7.60 & 1.90 & $\mathrm{pCi} / \mathrm{L}$ & 6.7 & Jet grout & 1134.3 & 283.6 & $\mathrm{pCi} / \mathrm{g}$ \\
\hline 14 Feb 92 & 37.60 & 2.60 & pCi/L & 11.8 & Jet grout & 3186.4 & 220.3 & $\mathrm{pCi} / \mathrm{g}$ \\
\hline 14 Feb 92 & 34.50 & 2.30 & $\mathrm{pCi} / \mathrm{L}$ & 11.0 & Jet grout & 3136.4 & 209.1 & $\mathrm{pCi} / \mathrm{s}$ \\
\hline 14 Feb 92 & 45.40 & 3.60 & $\mathrm{pCi} / \mathrm{L}$ & 20.2 & Jet grout & 2247.5 & 178.2 & $\mathrm{pCi} / \mathrm{s}$ \\
\hline 14 Feb 92 & 44.30 & 3.40 & $\mathrm{pCi} / \mathrm{L}$ & 19.7 & Jet grout & 2248.7 & 172.6 & $\mathrm{pCi} / \mathrm{g}$ \\
\hline 14 Feb 92 & 41.40 & 2.90 & $\mathrm{pCi} / \mathrm{L}$ & 16.4 & Jet grout & 2524.4 & 176.8 & $\mathrm{pCi} / \mathrm{s}$ \\
\hline 24 Feb 92 & 53.60 & 3.10 & $\mathrm{pCi} / \mathrm{L}$ & 17.4 & Jet grout & 3080.5 & 178.2 & $\mathrm{pCV} / \mathrm{g}$ \\
\hline 24 Feb 92 & 39.60 & 2.60 & $\mathrm{pCi} / \mathrm{L}$ & 14.8 & Jet grout & 2675.7 & 175.7 & $\mathrm{pCi} / \mathrm{g}$ \\
\hline 24 Feb 92 & 33.20 & 250 & pCir & 19.2 & Jet grout & 1729.2 & 130.2 & $\mathrm{pCi} / \mathrm{g}$ \\
\hline 24 Feb 92 & 38.8 & 3 & pCill & 16.4 & Jet grout & 2365.9 & 182.9 & $\mathrm{pCi} / \mathrm{s}$ \\
\hline 02 Mar 92 & 9.50 & 1.10 & $\mathrm{pCi} / \mathrm{L}$ & 6.2 & Jet grout & 1532.3 & 177.4 & $\mathrm{pCi} / \mathrm{s}$ \\
\hline $02 \operatorname{Mar} 92$ & 60.40 & 3.20 & $\mathrm{pCi} / \mathrm{L}$ & 20.0 & Jet grout & 3020.0 & 160.0 & $\mathrm{pCi} / \mathrm{g}$ \\
\hline 02 Mar 92 & 41.1 & 3.2 & $\mathrm{pCi} / \mathrm{L}$ & 19.6 & Jet grout & 2096.9 & 163.3 & $\mathrm{pCi} / \mathrm{g}$ \\
\hline 07 Mar 92 & 1.40 & 1.10 & $\mathrm{~Bq} / \mathrm{L}$ & & Jet grout & 37.8 & 29.7 & pCil \\
\hline 07 Mar 92 & 1.40 & 1.10 & $\mathrm{~Bq} / \mathrm{L}$ & & Jet grout & 37.8 & 29.7 & pCill \\
\hline 07 Mar 92 & 6.10 & 1.80 & $\mathrm{~Bq} / \mathrm{L}$ & & Jet grout & 164.9 & 48.7 & pCill \\
\hline 07 Mar 92 & 6.10 & 1.80 & $\mathrm{~Bq} / \mathrm{L}$ & & Jet grout & 164.9 & 48.7 & pCill \\
\hline 07 Mar 92 & 4.30 & 2.00 & $\mathrm{~Bq} / \mathrm{L}$ & & Jet grout & 116.2 & 54.1 & pCill \\
\hline 07 Mar 92 & 4.30 & 2.00 & $\mathrm{Bg} / \mathrm{L}$ & & Jet grout & 116.2 & 54.1 & pCill \\
\hline 07 Mar 92 & 6.30 & 1.60 & $\mathrm{~Bq} / \mathrm{L}$ & & Jet grout & 170.3 & 43.2 & pCill \\
\hline 07 Mar 92 & 6.30 & 1.60 & $\mathrm{~Bq} / \mathrm{L}$ & & Jet grout & 170.3 & 43.2 & $\mathrm{pCi} / \mathrm{L}$ \\
\hline $08 \mathrm{Mar} 92$ & 3.70 & 1.50 & $B q / L$ & & Jet grout & 100.0 & 40.5 & pCil \\
\hline
\end{tabular}


Table D.1 (continued)

\begin{tabular}{|c|c|c|c|c|c|c|c|c|}
\hline Date & $\begin{array}{l}{ }^{137} \mathrm{Cs} \\
\text { result }\end{array}$ & $\begin{array}{l}\text { Rad. } \\
\text { error }\end{array}$ & Units & $\begin{array}{c}\text { TSP } \\
(\mathrm{mg} / \mathrm{L})\end{array}$ & $\begin{array}{l}\text { Construction } \\
\text { activity }\end{array}$ & $\begin{array}{l}{ }^{137} \mathrm{Cs} \\
\text { result }\end{array}$ & $\begin{array}{l}\text { Rad. } \\
\text { error }\end{array}$ & Units \\
\hline $08 \mathrm{Mar} 92$ & 3.70 & 1.50 & $\mathrm{~Bq} / \mathrm{L}$ & & Jet grout & 100.0 & 40.5 & $\mathrm{pCi} / \mathrm{L}$ \\
\hline $08 \operatorname{Mar} 92$ & -0.40 & 1.40 & $\mathrm{~Bq} / \mathrm{L}$ & & Jet grout & -10.8 & 37.8 & $\mathrm{pCi} / \mathrm{L}$ \\
\hline $08 \mathrm{Mar} 92$ & -0.40 & 1.40 & $\mathrm{~Bq} / \mathrm{L}$ & & Jet grout & -10.8 & 37.8 & $\mathrm{pCi} / \mathrm{L}$ \\
\hline $08 \mathrm{Mar} 92$ & 1.70 & 1.30 & $\mathrm{~Bq} / \mathrm{L}$ & & Jet grout & 46.0 & 35.1 & $\mathrm{pC} / / \mathrm{L}$ \\
\hline $68 \mathrm{Mar} 92$ & 1.70 & 1.30 & $\mathrm{~Bq} / \mathrm{L}$ & & Jet grout & 46.0 & 35.1 & $\mathrm{pCi} / \mathrm{L}$ \\
\hline $08 \mathrm{Mar} 92$ & 1.20 & 0.80 & $\mathrm{~Bq} / \mathrm{L}$ & & Jet grout & 32.4 & 21.6 & pCi/L \\
\hline $08 \mathrm{Mar} 92$ & 1.20 & 0.80 & $\mathrm{~Bq} / \mathrm{L}$ & & Jet grout & 32.4 & 21.6 & $\mathrm{pCi} / \mathrm{L}$ \\
\hline $08 \operatorname{Mar} 92$ & 0.30 & 1.90 & $\mathrm{~Bq} / \mathrm{L}$ & & Jet grout & -8.1 & 51.4 & $\mathrm{pCi} / \mathrm{L}$ \\
\hline 08 Mar 92 & 0.30 & 1.90 & $\mathrm{~Bq} / \mathrm{L}$ & & Jet grout & -8.1 & 51.4 & pCilL \\
\hline 09 Mar 92 & 0.30 & 1.80 & $\mathrm{~Bq} / \mathrm{L}$ & & Jet grout & 8.1 & 48.7 & $\mathrm{pCi} / \mathrm{L}$ \\
\hline 09 Mar 92 & 0.30 & 1.80 & $\mathrm{~Bq} / \mathrm{L}$ & & Jet grout & 8.1 & 48.7 & pCil \\
\hline $09 \operatorname{Mar} 92$ & 1.40 & 1.50 & $\mathrm{~Bq} / \mathrm{L}$ & & Jet grout & 37.8 & 40.5 & $\mathrm{pCi} / \mathrm{L}$ \\
\hline $09 \operatorname{Mar} 92$ & 1.40 & 1.50 & $\mathrm{~Bq} / \mathrm{L}$ & & Jet grout & 37.8 & 40.5 & pCil \\
\hline $09 \operatorname{Mar} 92$ & -0.10 & 1.10 & $\mathrm{~Bq} / \mathrm{L}$ & & Jet grout & -2.7 & 29.7 & $\mathrm{pCi} / \mathrm{L}$ \\
\hline 09 Mar 92 & 4.80 & 1.40 & $\mathrm{~Bq} / \mathrm{L}$ & & Jet grout & 129.7 & 37.8 & pCi/L \\
\hline 09 Mar 92 & 4.80 & 1.40 & $\mathrm{~Bq} / \mathrm{L}$ & & Jet grout & 129.7 & 37.8 & $\mathrm{pCi} / \mathrm{L}$ \\
\hline $10 \operatorname{Mar} 92$ & 7.50 & 1.60 & $\mathrm{~Bq} / \mathrm{L}$ & & Jet grout & 202.7 & 43.2 & $\mathrm{pCi} / \mathrm{L}$ \\
\hline $10 \operatorname{Mar} 92$ & 7.50 & 1.60 & $\mathrm{~Bq} / \mathrm{L}$ & & Jet grout & 202.7 & 43.2 & $\mathrm{pCi} / \mathrm{L}$ \\
\hline $10 \operatorname{Mar} 92$ & 9.80 & 1.80 & $\mathrm{~Bq} / \mathrm{L}$ & & Jet grout & 264.9 & 48.7 & pCill \\
\hline $10 \operatorname{Mar} 92$ & 9.80 & 1.80 & $\mathrm{~Bq} / \mathrm{L}$ & & Jet grout & 264.9 & 48.7 & $\mathrm{pCi} / \mathrm{L}$ \\
\hline $10 \operatorname{Mar} 92$ & 11.00 & 2.00 & $\mathrm{~Bq} / \mathrm{L}$ & & Jet grout & 297.3 & 54.1 & pCil \\
\hline $10 \operatorname{Mar} 92$ & 11.00 & 2.00 & $\mathrm{~Bq} / \mathrm{L}$ & & Jet grout & 297.3 & 54.1 & $\mathrm{pCi} / \mathrm{L}$ \\
\hline 11 Mar 92 & 11.00 & 2.00 & $\mathrm{~Bq} / \mathrm{L}$ & & Jet grout & 297.3 & 54.1 & pCi/L \\
\hline $11 \operatorname{Mar} 92$ & 11.00 & 2.00 & $\mathrm{~Bq} / \mathrm{L}$ & & Jet grout & 297.3 & 54.1 & $\mathrm{pCil}$ \\
\hline 11 Mar 92 & 12.00 & 2.00 & $\mathrm{~Bq} / \mathrm{L}$ & & Jet grout & 324.4 & 54. & $\mathrm{pCi} / \mathrm{L}$ \\
\hline 11 Mar 92 & 12.00 & 2.00 & $\mathrm{~Bq} / \mathrm{L}$ & & Jet grout & 324.4 & 54.1 & $\mathrm{pCi} / \mathrm{L}$ \\
\hline $11 \operatorname{Mar} 92$ & 12.00 & 2.00 & $\mathrm{~Bq} / \mathrm{L}$ & & Jet grout & 324.4 & 54.1 & pCill. \\
\hline $11 \operatorname{Mar} 92$ & 12.00 & 2.00 & $\mathrm{~Bq} / \mathrm{L}$ & & Jet grout & 324.4 & 54.1 & $\mathrm{pCi} / \mathrm{L}$ \\
\hline $12 \operatorname{Mar} 92$ & 4.90 & 1.70 & $\mathrm{~Bq} / \mathrm{L}$ & & Jet grout & 132.4 & 46.0 & pCi/L \\
\hline $12 \operatorname{Mar} 92$ & 4.90 & 1.70 & $\mathrm{~Bq} / \mathrm{L}$ & & Jet grout & 132.4 & 46.0 & pCir \\
\hline $12 \operatorname{Mar} 92$ & 4.20 & 1.40 & $\mathrm{~Bq} / \mathrm{L}$ & & Jet grout & 113.5 & 37.8 & $\mathrm{pCi} / \mathrm{L}$ \\
\hline $12 \operatorname{Mar} 92$ & 4.20 & 1.40 & $\mathrm{~Bq} / \mathrm{L}$ & & Jet grout & 113.5 & 37.8 & $\mathrm{pCi} / \mathrm{L}$ \\
\hline 16 Mar 92 & 0.10 & 2.40 & $\mathrm{~Bq} / \mathrm{L}$ & & Jet grout & 2.7 & 64.9 & $\mathrm{pCi} / \mathrm{L}$ \\
\hline
\end{tabular}


Table D.1 (continued)

\begin{tabular}{|c|c|c|c|c|c|c|c|c|}
\hline Date & $\begin{array}{l}{ }^{137} \mathrm{Cs} \\
\text { result }\end{array}$ & $\begin{array}{l}\text { Rad. } \\
\text { error }\end{array}$ & Units & $\begin{array}{c}\mathrm{TSP} \\
(\mathrm{mg} / \mathrm{L})\end{array}$ & $\begin{array}{l}\text { Construction } \\
\text { activity }\end{array}$ & $\begin{array}{l}{ }^{137} \mathrm{Cs} \\
\text { result }\end{array}$ & $\begin{array}{l}\text { Rad. } \\
\text { error }\end{array}$ & Units \\
\hline $16 \operatorname{Mar} 92$ & 0.10 & 2.40 & $\mathrm{~Bq} / \mathrm{L}$ & & Jet grout & 2.7 & 64.9 & $\mathrm{pCi} / \mathrm{L}$ \\
\hline $16 \operatorname{Mar} 92$ & 1.50 & 1.70 & $\mathrm{~Bq} / \mathrm{L}$ & & Jet grout & 40.5 & 46.0 & $\mathrm{pCi} / \mathrm{L}$ \\
\hline $16 \operatorname{Mar} 92$ & 1.50 & 1.70 & $\mathrm{~Bq} / \mathrm{L}$ & & Jet grout & 40.5 & 46.0 & $\mathrm{pCi} / \mathrm{L}$ \\
\hline $16 \operatorname{Mar} 92$ & 0.10 & 2.10 & $\mathrm{~Bq} / \mathrm{L}$ & & Jet grout & 2.7 & 56.8 & $\mathrm{pCi} / \mathrm{L}$ \\
\hline $16 \operatorname{Mar} 92$ & 0.10 & 2.10 & $\mathrm{~Bq} / \mathrm{L}$ & & Jet grout & 2.7 & 56.8 & $\mathrm{pCi} / \mathrm{L}$ \\
\hline 20 Mar 92 & 7.40 & 1.20 & $\mathrm{~Bq} / \mathrm{L}$ & & Jet grout & 200.0 & 32.4 & $\mathrm{pCi} / \mathrm{L}$ \\
\hline $21 \operatorname{Mar} 92$ & 5.90 & 1.50 & $\mathrm{~Bq} / \mathrm{L}$ & & Jet grout & 159.5 & 40.5 & $\mathrm{pCi} / \mathrm{L}$ \\
\hline $21 \mathrm{Mar} 92$ & 1.10 & 0.40 & $\mathrm{~Bq} / \mathrm{L}$ & & Jet grout & 29.7 & 10.8 & pCi/L \\
\hline $23 \operatorname{Mar} 92$ & 28.7 & 1.5 & $\mathrm{pCi} / \mathrm{L}$ & 16.5 & Jet grout & 1739.4 & 90.9 & $\mathrm{pCi} / \mathrm{g}$ \\
\hline 23 Mar 92 & 29.1 & 1.6 & pCi/L & 16.5 & Jet grout & 1763.6 & 97.0 & pCi/g \\
\hline $24 \operatorname{Mar} 92$ & 1.90 & 1.10 & $\mathrm{~Bq} / \mathrm{L}$ & & Jet grout & 51.4 & 29.7 & pCil \\
\hline $24 \mathrm{Mar} 92$ & 2.10 & 1.10 & $\mathrm{~Bq} / \mathrm{L}$ & & Jet grout & 56.8 & 29.7 & pCi/L \\
\hline $21 \operatorname{Mar} 92$ & 4.20 & 1.20 & $\mathrm{~Bq} / \mathrm{L}$ & & Jet grout & 113.5 & 2.4 & $\mathrm{PCiL}$ \\
\hline 25 Mar 92 & 1.80 & 2.60 & $\mathrm{~Bq} / \mathrm{L}$ & & Jet grout & 48.7 & 70.3 & pCiL \\
\hline $25 \operatorname{Mar} 92$ & 0.40 & 3.10 & $\mathrm{~Bq} / \mathrm{L}$ & & Jet grout & 10.8 & 83.8 & pCiL \\
\hline $26 \mathrm{Mar} 92$ & 5.80 & 2.50 & $\mathrm{~Bq} / \mathrm{L}$ & & Dredging & 156.8 & 67.6 & $\mathrm{pCiL}$ \\
\hline 26 Mar 92 & 158.3 & 5.1 & $\mathrm{pCi} / \mathrm{L}$ & 47.0 & Dredging & 3368.1 & 108.5 & $\mathrm{pCi} / \mathrm{g}$ \\
\hline $26 \operatorname{Mar} 92$ & 161 & 5.3 & $\mathrm{pCi} / \mathrm{L}$ & 47.0 & Dredging & 3425.5 & 112.8 & $\mathrm{pCi} / \mathrm{g}$ \\
\hline $26 \operatorname{Mar} 92$ & 0.40 & 3.90 & $B q / L$ & & Dredging & 10.8 & 105.4 & pCil \\
\hline $26 \operatorname{Mar} 92$ & 217. & 10. & $\begin{array}{l}\text { Total } \\
\mathrm{pCi}\end{array}$ & 22.0 & Dredging & 0.0 & 0.0 & $\mathrm{pCi} / \mathrm{g}$ \\
\hline $26 \mathrm{Mar} 92$ & 49.2 & 2.4 & Total & 22.0 & Dredging & 2236.4 & 109.1 & pCi/s \\
\hline 26 Mar 92 & 12.00 & 3.00 & $B q / L$ & & Dredging & 324.4 & 81.1 & pCin \\
\hline 30 Mar 92 & 17.3 & 1.5 & $\mathrm{pCi} / \mathrm{L}$ & 11.5 & & 1504.3 & 130.4 & $\mathrm{pC} / \mathrm{g}$ \\
\hline $30 \operatorname{Mar} 92$ & 18.3 & 1.4 & & 11.5 & & 1591.3 & 121.7 & pCi/g \\
\hline 31 Mar 92 & 2.00 & 3.30 & $\mathrm{~Bq} / \mathrm{L}$ & & Capping & 54.1 & 89.2 & pCi/L \\
\hline 31 Mar 92 & 0.30 & 3.10 & $\mathrm{~Bq} / \mathrm{L}$ & & Capping & 8.1 & 83.8 & pCil \\
\hline $31 \operatorname{Mar} 92$ & -0.10 & 3.50 & $\mathrm{Bg} / \mathrm{L}$ & & Capping & -2.7 & 94.6 & $\mathrm{pCi} / \mathrm{L}$ \\
\hline 01 Apr 92 & 0.60 & 2.10 & $\mathrm{~Bq} / \mathrm{L}$ & & Dredging & 16.2 & 56.8 & pCil \\
\hline 01 Apr 92 & $\mathbf{0}$ & & $\mathrm{pCi} / \mathrm{L}$ & 3.4 & Dredging & 0.0 & 0.0 & $\mathrm{pCi} / \mathrm{g}$ \\
\hline 01 Apr 92 & 0.70 & 2.10 & $\mathrm{~Bq} / \mathrm{L}$ & & Dredging & 18.9 & 56.8 & pCi/L \\
\hline 01 Apr 92 & 3.1 & 1.3 & $\mathrm{pCi} / \mathrm{L}$ & 3.9 & Dredging & 794.9 & 333.3 & $\mathrm{pCi} / \mathrm{g}$ \\
\hline 01 Apr 92 & 2.60 & 1.80 & $\mathrm{~Bq} / \mathrm{L}$ & & Dredging & 70.3 & 48.7 & $\mathrm{pCi} / \mathrm{L}$ \\
\hline 01 Apr 92 & 22.7 & 2.2 & $\mathrm{pCi} / \mathrm{L}$ & 18.5 & Dredging & 1227.0 & 118.9 & $\mathrm{pCi} / \mathrm{g}$ \\
\hline
\end{tabular}


D-6

Table D.1 (continued)

\begin{tabular}{|c|c|c|c|c|c|c|c|c|}
\hline Date & $\begin{array}{l}{ }^{137} \mathrm{Cs} \\
\text { result }\end{array}$ & $\begin{array}{l}\text { Rad. } \\
\text { error }\end{array}$ & Units & $\begin{array}{c}\text { TSP } \\
(\mathrm{mg} / \mathrm{L})\end{array}$ & $\begin{array}{l}\text { Construction } \\
\text { activity }\end{array}$ & $\begin{array}{l}{ }^{137} \mathrm{Cs} \\
\text { result }\end{array}$ & $\begin{array}{l}\text { Rad. } \\
\text { error }\end{array}$ & Units \\
\hline 01 Apr 92 & 3.00 & 1.60 & $\mathrm{~Bq} / \mathrm{L}$ & & Dredging & 81.1 & 43.2 & $\mathrm{pCi} / \mathrm{L}$ \\
\hline 01 Apr 92 & 274.5 & 7.5 & $\mathrm{pCi} / \mathrm{L}$ & 38.4 & Dredging & 7148.4 & 195.3 & $\mathrm{pCi} / \mathrm{g}$ \\
\hline 01 Apr 92 & 263.8 & 6.7 & & 38.4 & Dredging & 6869.8 & 174.5 & $\mathrm{pCi} / \mathrm{g}$ \\
\hline 01 Apr 92 & 1.70 & 1.50 & $\mathrm{~Bq} / \mathrm{L}$ & & Dredging & 46.0 & 40.5 & $\mathrm{pCi} / \mathrm{L}$ \\
\hline 01 Apr 92 & 64.6 & 3.1 & $\mathrm{pCi} / \mathrm{L}$ & 23.7 & Dredging & 2725.7 & 130.8 & $\mathrm{pCi} / \mathrm{g}$ \\
\hline 01 Apr 92 & 76.7 & 3.4 & & 23.7 & Dredging & 3236.3 & 143.5 & $\mathrm{pCi} / \mathrm{g}$ \\
\hline 04 Apr 92 & 2.00 & 1.90 & $\mathrm{~Bq} / \mathrm{L}$ & & Drilling & 54.1 & 51.4 & $\mathrm{pCi} / \mathrm{L}$ \\
\hline 04 Apr 92 & 27 & 1.8 & $\mathrm{pCi} / \mathrm{L}$ & 8.5 & Drilling & 3176.5 & 211.8 & $\mathrm{pCi} / \mathrm{g}$ \\
\hline 06 Apr 92 & 1.20 & .80 & $\mathrm{~Bq} / \mathrm{L}$ & & Drilling & 32.4 & 48.7 & $\mathrm{pCi} / \mathrm{L}$ \\
\hline 06 Apr 92 & 5.1 & 1.1 & $\mathrm{pCi} / \mathrm{L}$ & 6.5 & Drilling & 784.6 & 169.2 & $\mathrm{pCi} / \mathrm{g}$ \\
\hline 06 Apr 92 & -0.40 & 2.40 & $\mathrm{~Bq} / \mathrm{L}$ & & Drilling & -10.8 & 64.9 & $\mathrm{pCi} / \mathrm{L}$ \\
\hline 06 Apr 92 & 3.3 & 1.3 & $\mathrm{pCi} / \mathrm{L}$ & 8.1 & Drilling & 407.4 & 160.5 & $\mathrm{pC} / \mathrm{g}$ \\
\hline 06 Apr 92 & 312 & 10.9 & $\mathrm{pCi} / \mathrm{L}$ & 51.0 & Drilling & 6117.6 & 213.7 & $\mathrm{pCi} / \mathrm{g}$ \\
\hline 06 Apr 92 & 16.9 & 2.2 & $\mathrm{pCi} / \mathrm{L}$ & 5.7 & Drilling & 2964.9 & 386.0 & $\mathrm{pCi} / \mathrm{g}$ \\
\hline 06 Apr 92 & 14.7 & 1.6 & $\mathrm{pCi} / \mathrm{L}$ & 5.7 & Drilling & 2578.9 & 280.7 & $\mathrm{pCi} / \mathrm{g}$ \\
\hline 06 Apr 92 & 18 & 1.7 & $\mathrm{pCi} / \mathrm{L}$ & 6.0 & Drilling & 3000.0 & 283.3 & $\mathrm{pCi} / \mathrm{g}$ \\
\hline 06 Apr 92 & 41.2 & 2.8 & $\mathrm{pCi} / \mathrm{L}$ & 21.6 & Drilling & 1907.4 & 129.6 & $\mathrm{pCi} / \mathrm{g}$ \\
\hline 08 Apr 92 & 1.30 & 1.30 & $\mathrm{~Bq} / \mathrm{L}$ & & Drilling & 35.1 & 35.1 & pCi/L \\
\hline 08 Apr 92 & 8.7 & 1.8 & $\mathrm{pCi} / \mathrm{L}$ & 9.6 & Drilling & 906.3 & 87.5 & $\mathrm{pCi} / \mathrm{g}$ \\
\hline 08 Apr 92 & 50.00 & 2.00 & $\mathrm{~Bq} / \mathrm{L}$ & & Drilling & 1351.5 & 54.1 & pCil \\
\hline 08 Apr 92 & 320 & 6.6 & $\mathrm{pCi} / \mathrm{L}$ & 100.4 & Drilling & 3187.3 & 65.7 & $\mathrm{pCi} / \mathrm{g}$ \\
\hline 08 Apr 92 & 5.50 & 1.30 & $\mathrm{~Bq} / \mathrm{L}$ & & Drilling & 148.7 & 35.1 & $\mathrm{pCi} / \mathrm{L}$ \\
\hline 08 Apr 92 & 36.6 & 3.1 & $\mathrm{pCi} / \mathrm{L}$ & & Drilling & 0.0 & 0.0 & $\mathrm{pCi} / \mathrm{g}$ \\
\hline 08 Apr 92 & -0.20 & 1.60 & $\mathrm{~Bq} / \mathrm{L}$ & & Drilling & -5.4 & 43.2 & $\mathrm{pCi} / \mathrm{L}$ \\
\hline 08 Apr 92 & 26.00 & 2.00 & $\mathrm{~Bq} / \mathrm{L}$ & & Drilling & 702.8 & 54.1 & $\mathrm{pCi} / \mathrm{L}$ \\
\hline 08 Apr 92 & 211.8 & 5.4 & $\mathrm{pCi} / \mathrm{L}$ & & Drilling & 0.0 & 0.0 & $\mathrm{pC} / \mathrm{g}$ \\
\hline 08 Apr 92 & 88.00 & 3.00 & $\mathrm{~Bq} / \mathrm{L}$ & & Drilling & 2378.6 & 81.1 & $\mathrm{pCi} / \mathrm{L}$ \\
\hline 08 Apr 92 & 598.5 & 11.5 & $\mathrm{pCi} / \mathrm{L}$ & & Drilling & 0.0 & 0.0 & $\mathrm{pCi} / \mathrm{g}$ \\
\hline 08 Apr 92 & 47.00 & 2.00 & $\mathrm{~Bq} / \mathrm{L}$ & & Drilling & 1270.4 & 54. & $\mathrm{pCi} / \mathrm{L}$ \\
\hline 08 Apr 92 & 355 & 7.1 & $\mathrm{pCi} / \mathrm{L}$ & & Drilling & 0.0 & 0.0 & $\mathrm{pCi} / \mathrm{g}$ \\
\hline 09 Apr 92 & 10.7 & 1.8 & $\mathrm{pCi} / \mathrm{L}$ & 8.1 & Drilling & 1321.0 & 222.2 & $\mathrm{pCi} / \mathrm{g}$ \\
\hline 09 Apr 92 & 432.4 & 10.3 & $\mathrm{pCi} / \mathrm{L}$ & 64.2 & Drilling & 6735.2 & 160.4 & $\mathrm{pCi} / \mathrm{g}$ \\
\hline 09 Apr 92 & 162.4 & 5.4 & $\mathrm{pCi} / \mathrm{L}$ & 51.3 & Drilling & 3165.7 & 105.3 & pCi/g \\
\hline
\end{tabular}


D-7

Table D.1 (continued)

\begin{tabular}{ccccccccc}
\hline Date & $\begin{array}{c}{ }^{137} \mathrm{Cs} \\
\text { result }\end{array}$ & $\begin{array}{c}\text { Rad. } \\
\text { error }\end{array}$ & Units & $\begin{array}{c}\mathrm{TSP} \\
(\mathrm{mg} / \mathrm{L})\end{array}$ & $\begin{array}{c}\text { Construction } \\
\text { activity }\end{array}$ & $\begin{array}{r}{ }^{137} \mathrm{Cs} \\
\text { result }\end{array}$ & $\begin{array}{r}\text { Rad. } \\
\text { error }\end{array}$ & Units \\
\hline $10 \mathrm{Apr} 92$ & 626.4 & 10.5 & $\mathrm{pCi} / \mathrm{L}$ & 102.7 & Drilling & 6099.3 & 102.2 & $\mathrm{pCi} / \mathrm{g}$ \\
$10 \mathrm{Apr} 92$ & 626.4 & 10.6 & $\mathrm{pCi} / \mathrm{L}$ & 102.7 & Drilling & 6099.3 & 103.2 & $\mathrm{pCi} / \mathrm{g}$ \\
$10 \mathrm{Apr} 92$ & 38.8 & 2.8 & $\mathrm{pCi} / \mathrm{L}$ & 39.9 & Drilling & 972.4 & 70.2 & $\mathrm{pCi} / \mathrm{g}$ \\
$10 \mathrm{Apr} 92$ & 293.3 & 6.2 & $\mathrm{pCi} / \mathrm{L}$ & 64.6 & Drilling & 4540.2 & 96.0 & $\mathrm{pCi} / \mathrm{g}$ \\
$10 \mathrm{Apr} 92$ & 13 & 1.9 & $\mathrm{pCi} / \mathrm{L}$ & 12.5 & Drilling & 1040.0 & 152.0 & $\mathrm{pCi} / \mathrm{g}$ \\
\hline
\end{tabular}


Appendix E QUALTY ASSURANCE/QUALITY CONTROL DATA 


\section{E-3}

Table E.1. Project quality assurance/quality control data for 24h composite samples. Match these values with those for Appendix A.

\begin{tabular}{|c|c|c|c|c|c|c|c|c|c|}
\hline Sample & I.D. & Date & $\begin{array}{c}\text { River } \\
\text { mile }\end{array}$ & Analysis & Result & Error & Units & $\begin{array}{c}\text { QC sample } \\
\text { type }\end{array}$ & $\begin{array}{c}\text { Construction } \\
\text { phase }\end{array}$ \\
\hline 629 & $\mathbf{z}$ & 18 Sep 91 & 20.8 & Turbidity & 1.50 & & NTU & Duplicate & Rock armor \\
\hline 631 & $\mathbf{Z}$ & $19 \operatorname{Sep} 91$ & 20.8 & Tot. susp. solids & 5.00 & & $\mathbf{m g} / \mathbf{L}$ & Duplicate & Rock armor \\
\hline 631 & $\mathbf{Z}$ & $19 \operatorname{Sep} 91$ & 20.8 & Turbidity & 1.10 & & NTU & Duplicate & Rock armor \\
\hline 632 & $\mathbf{z}$ & $19 \operatorname{Sep} 91$ & 14.5 & Turbidity & 1.50 & & NTU & Duplicate & Rock armor \\
\hline 632 & $\mathbf{Z}$ & $19 \operatorname{Sep} 91$ & 14.5 & Tot. susp. solids & 14.00 & & $\mathrm{mg} / \mathrm{L}$ & Duplicate & Rock armor \\
\hline 633 & $\mathbf{z}$ & 20 Sep 91 & 20.8 & Turbidity & 1.90 & & NTU & Duplicate & Rock armor \\
\hline 633 & $\mathbf{Z}$ & $20 \operatorname{Sep} 91$ & 20.8 & Tot. susp. solids & 5.00 & & $\mathrm{mg} / \mathrm{L}$ & Duplicale & Rock armor \\
\hline 633 & $\mathbf{G}$ & $20 \operatorname{Sep} 91$ & 20.8 & ${ }^{19} \mathrm{Cs}$ & 0.04 & 0.84 & $\mathrm{~Bq} / \mathrm{L}$ & Rinse water & Rock armor \\
\hline 634 & $\mathbf{z}$ & 23 Sep 91 & 20.8 & Turbidity & 1.30 & & NTU & Duplicate & Rock armor \\
\hline 634 & $\mathbf{z}$ & $23 \operatorname{Sep} 91$ & 20.8 & Tot. susp. solids & 5.00 & & $\mathrm{mgl}$ & Duplicate & Rock armor \\
\hline 635 & $\mathbf{z}$ & $24 \operatorname{Sep} 91$ & 20.8 & Turbidity & 1.70 & & NTU & Duplicate & Rock armor \\
\hline 635 & $\mathbf{Z}$ & $24 \operatorname{Sep} 91$ & 20.8 & Tot. susp. solids & 5.00 & & $\mathrm{mg} / \mathrm{L}$ & Duplicate & Rock armor \\
\hline 636 & $\mathbf{Z}$ & $24 \operatorname{Sep} 91$ & 14.5 & Turbidity & 1.70 & & NTU & Duplicate & Rock armor \\
\hline 636 & $\mathbf{z}$ & $24 \operatorname{Sep} 91$ & 14.5 & Tot. susp. solids & 5.00 & & $\mathrm{~m} / \mathrm{L}$ & Duplicate & Rock armor \\
\hline 643 & $\mathbf{Z}$ & $25 \operatorname{Sep} 91$ & 20.8 & Tot. susp. solids & 5.00 & & $\mathrm{mg} / \mathrm{L}$ & Duplicate & Rock armor \\
\hline 643 & $\mathbf{z}$ & 25 Sep 91 & 20.8 & Turbidity & 2.00 & & NTU & Duplicate & Rock armor \\
\hline 644 & $\mathbf{z}$ & 25 Sep 91 & 14.5 & Tot. susp. solids & 5.00 & & $\mathrm{mg} / \mathrm{L}$ & Duplicate & Rock armor \\
\hline 644 & $\mathbf{Z}$ & $25 \operatorname{Sep} 91$ & 14.5 & Turbidity & 1.40 & & NTU & Duplicate & Rock armor \\
\hline 645 & $\mathbf{Z}$ & $26 \operatorname{Sep} 91$ & 20.8 & Tot. susp. solids & 5.00 & & $\mathrm{mg} / \mathrm{L}$ & Duplicate & Rock armor \\
\hline 645 & $\mathbf{z}$ & $26 \operatorname{Sep} 91$ & 20.8 & Turbidity & 2.20 & & NTU & Duplicate & Rock armor \\
\hline 646 & $\mathbf{Z}$ & $26 \operatorname{Sep} 91$ & 14.5 & Turbidity & 1.50 & & NTU & Duplicate & Rock armor \\
\hline 646 & $\mathbf{Z}$ & $26 \operatorname{Sep} 91$ & 14.5 & Tot. susp. solids & 5.00 & & $\mathrm{mg} / \mathrm{L}$ & Duplicate & Rock armor \\
\hline 647 & $\mathbf{Z}$ & 27 Sep 91 & 20.8 & Tot. susp. solids & 5.00 & & $\mathrm{mg} / \mathrm{L}$ & Duplicate & Rock armor \\
\hline 647 & $\mathbf{G}$ & 27 Sep 91 & 20.8 & ${ }^{17} \mathrm{Cs}$ & 0.10 & 1.20 & $\mathrm{Bg} / \mathrm{L}$ & Rinse water & Rock armor \\
\hline 647 & $\mathbf{Z}$ & 27 Sep 91 & 20.8 & Turbidity & 2.40 & & NTU & Duplicate & Rock armor \\
\hline 648 & $\mathbf{Z}$ & $27 \operatorname{Sep} 91$ & 14.5 & Turbidity & 2.00 & & NTU & Duplicate & Rock armor \\
\hline 648 & G & $27 \operatorname{Sep} 91$ & 14.5 & ${ }^{n} \mathrm{Cs}$ & -0.11 & 0.75 & $\mathrm{Bg} / \mathrm{L}$ & Rinse water & Rock armor \\
\hline 648 & $\mathbf{Z}$ & $27 \operatorname{Sep} 91$ & 14.5 & Tot. susp. solids & 5.00 & & $m g / L$ & Duplicate & Rock armor \\
\hline 649 & $\mathbf{z}$ & $30 \operatorname{Sep} 91$ & 20.8 & Turbidity & 1.30 & & NTU & Duplicate & Rock armor \\
\hline 649 & $\mathbf{Z}$ & $30 \operatorname{Sep} 91$ & 20.8 & Tot. susp. solids & 5.00 & & $\mathbf{m g} / \mathbf{L}$ & Duplicate & Rock armor \\
\hline 651 & $\mathbf{Z}$ & $30 \operatorname{Sep} 91$ & 14.5 & Tot. susp. solids & 5.00 & & $\mathrm{mg} / \mathrm{L}$ & Duplicale & Reck armor \\
\hline 651 & $\mathbf{Z}$ & 30 Sep 91 & 14.5 & Turbidity & 1.10 & & NTU & Duplicate & Rock armor \\
\hline 652 & $\mathbf{Z}$ & 01 Oct 91 & 20.8 & Turbidity & 1.45 & & NTU & Duplicate & Rock armor \\
\hline 652 & $\mathbf{z}$ & 01 Oct 91 & 20.8 & Tot. susp. solids & 5.00 & & $\mathrm{mg} / \mathrm{L}$ & Duplicate & Rock armor \\
\hline
\end{tabular}


Table E1 (continued)

\begin{tabular}{|c|c|c|c|c|c|c|c|c|c|}
\hline Sample & I.D. & Date & $\begin{array}{c}\text { River } \\
\text { mile }\end{array}$ & Analysis & Result & Error & Units & $\begin{array}{c}\text { QC sample } \\
\text { type }\end{array}$ & $\begin{array}{c}\text { Construction } \\
\text { phase }\end{array}$ \\
\hline 653 & $\mathbf{Z}$ & 01 Oct 91 & 14.5 & Tot. susp. solids & 5.00 & & $\mathrm{mg} / \mathrm{L}$ & Duplicate & Rock armor \\
\hline 653 & $\mathbf{Z}$ & 01 Oct 91 & 14.5 & Tot. susp. solids & 5.00 & & $\mathrm{mg} / \mathrm{L}$ & Duplicate & Rock armor \\
\hline 653 & $\mathbf{Z}$ & 01 Oct 91 & 14.5 & Turbidity & 1.50 & & NTU & Duplicate & Rock armor \\
\hline 677 & $\mathbf{z}$ & 03 Oct 91 & 20.8 & Tot. susp. solids & 5.00 & & $\mathrm{mg} / \mathrm{L}$ & Duplicate & Rock armor \\
\hline 677 & $\mathbf{z}$ & 03 Oct 91 & 20.8 & Turbidity & 1.20 & & NTU & Duplicate & Rock armor \\
\hline 678 & $\mathbf{z}$ & 03 Oct 91 & 14.5 & Turbidity & 1.00 & & NTU & Duplicate & Rock armor \\
\hline 678 & $\mathbf{z}$ & 03 Oct 91 & 14.5 & Tot. susp. solids & 5.00 & & $\mathbf{m g} / \mathbf{L}$ & Duplicate & Rock armor \\
\hline 678 & $\mathbf{z}$ & 03 Oct 91 & 14.5 & Tot. susp. solids & 5.00 & & $\mathrm{mg} / \mathrm{L}$ & Duplicate & Rock armor \\
\hline 679 & $\mathbf{G}$ & 04 Oct 91 & 20.8 & ${ }^{1 n} \mathrm{Cs}$ & 0.09 & 0.51 & $\mathrm{~Bq} / \mathrm{L}$ & Rinse water & Rock armor \\
\hline 679 & $\mathbf{z}$ & 04 Oct 91 & 20.8 & Tot. susp. solids & 5.00 & & $m g / L$ & Duplicate & Rock armor \\
\hline 679 & $\mathbf{z}$ & 04 Oct 91 & 20.8 & Turbidity & 1.50 & & NTU & Duplicate & Rock armor \\
\hline 681 & $\mathbf{z}$ & 04 Oct 91 & 14.5 & Turbidity & 2.40 & & NTU & Duplicate & Rock armor \\
\hline 681 & $\mathbf{Z}$ & 04 Oct 91 & 14.5 & Tot. susp. solids & 5.00 & & $\mathrm{mg} / \mathrm{L}$ & Duplicate & Rock armor \\
\hline 681 & $\mathbf{G}$ & 04 Oct 91 & 14.5 & ${ }^{n} \mathrm{Cs}$ & -0.06 & 0.75 & $\mathrm{Bg} / \mathrm{L}$ & Rinse water & Rock armor \\
\hline 682 & $\mathbf{z}$ & 07 Oct 91 & 20.8 & Turbidity & 1.00 & & NTU & Duplicate & Rock armor \\
\hline 682 & $\mathbf{z}$ & 07 Oct 91 & 20.8 & Tot. susp. solids & 5.00 & & $\mathrm{mg} / \mathrm{L}$ & Duplicate & Rock armor \\
\hline 683 & $\mathbf{z}$ & 07 Oct 91 & 14.5 & Turbidity & 0.62 & & NTU & Duplicate & Rock armor \\
\hline 683 & $\mathbf{z}$ & 07 Oct 91 & 14.5 & Tot. susp. solids & 5.00 & & $m g / L$ & Duplicate & Rock armor \\
\hline 685 & $\mathbf{z}$ & 08 Oct 91 & 14.5 & Tot. susp. solids & 5.00 & & $m g / L$ & Duplicate & Rock armor \\
\hline 685 & $\mathbf{z}$ & 08 Oct 91 & 14.5 & Turbidity & 1.50 & & NTU & Duplicate & Rock armor \\
\hline 744 & $\mathbf{z}$ & 29 Oct 91 & 20.8 & Turbiditv & 0.80 & & NTU & Duplicate & \\
\hline 744 & $\mathbf{z}$ & 29 Oct 91 & 20.8 & Tot. susp. solids & 2.00 & & $\mathrm{mg} / \mathrm{L}$ & Duplicate & \\
\hline 745 & $\mathbf{z}$ & 29 Oct 91 & 14.5 & Tot. susp. solids & 5.00 & & $\mathrm{mg} / \mathrm{L}$ & Duplicate & \\
\hline 745 & $\mathbf{z}$ & 29 Oct 91 & 14.5 & Turbidity & 1.00 & & NTU & Duplicate & \\
\hline 746 & $\mathbf{Z}$ & 30 Oct 91 & 20.8 & Turbidity & 2.90 & & NTU & Duplicate & \\
\hline 746 & $\mathbf{z}$ & 30 Oct 91 & 20.8 & Tot. susp. solids & 5.00 & & $\operatorname{mgl}$ & Duplicate & \\
\hline 747 & $\mathbf{z}$ & 31 Oct 91 & 20.8 & Tot. susp. solids & 5.00 & & $\mathrm{mg} / \mathrm{L}$ & Duplicate & \\
\hline 747 & $\mathbf{Z}$ & 31 Oct 91 & 20.8 & Turbidity & 1.10 & & NTU & Duplicate & \\
\hline 748 & $\mathbf{z}$ & 31 Oct 91 & 14.5 & Turbidity & 1.50 & & NTU & Duplicate & \\
\hline 748 & $\mathbf{Z}$ & 31 Oct 91 & 14.5 & Tot. susp. solids & 5.00 & & $m g /$ & Duplicate & \\
\hline 749 & $\mathbf{z}$ & 01 Now 91 & 20.8 & Turbidity & 1.30 & & NTU & Duplicate & \\
\hline 749 & $\mathbf{Z}$ & 01 Nov 91 & 20.8 & Tot. susp. solids & 5.00 & & $\mathrm{mg} / \mathrm{L}$ & Duplicate & \\
\hline 749 & $\mathbf{G}$ & 01 Nov 91 & 20.8 & ${ }^{17} \mathrm{Cs}$ & 0.06 & 0.48 & $\mathrm{~Bq} / \mathrm{L}$ & Rinse water & \\
\hline 751 & $\mathbf{z}$ & 04 Nov 91 & 20.8 & Turbidity & 1.10 & & NTU & Duplicate & \\
\hline 751 & $\mathbf{Z}$ & O4 Now 91 & 20.8 & Tot. susp. solids & 5.00 & & $\mathrm{mg} / \mathrm{L}$ & Duplicate & \\
\hline
\end{tabular}


E-5

Table E.1 (continued)

\begin{tabular}{|c|c|c|c|c|c|c|c|c|}
\hline & Date & $\begin{array}{l}\text { River } \\
\text { mile }\end{array}$ & Analysis & Result & Error & Units & $\begin{array}{l}\text { QC sample } \\
\text { type }\end{array}$ & $\begin{array}{c}\text { Construction } \\
\text { phase }\end{array}$ \\
\hline $\mathbf{z}$ & 05 Nov 91 & 20.8 & Tot. susp. solids & 10.00 & & $\mathbf{m g} / \mathbf{L}$ & Duplicate & \\
\hline $\mathbf{z}$ & 05 Nov 91 & 20.8 & Turbidity & 2.70 & & NTU & Duplicate & \\
\hline $\mathbf{Z}$ & 05 Nov 91 & 14.5 & Turbidity & 2.50 & & NTU & Duplicate & \\
\hline $\mathbf{Z}$ & 05 Nov 91 & 14.5 & Tot. susp. solids & 6.00 & & $\mathrm{mg} / \mathrm{L}$ & Duplicate & \\
\hline $\mathbf{Z}$ & 06 Now 91 & 20.8 & Turbidity & 3.30 & & NTU & Duplicate & \\
\hline $\mathbf{Z}$ & O6 Nov 91 & 20.8 & Tot. susp. solids & 27.00 & & $\mathrm{mg} / \mathrm{L}$ & Duplicate & \\
\hline $\mathbf{Z}$ & 06 Nov 91 & 20.8 & Turbidity & 1.50 & & NTU & Duplicate & \\
\hline $\mathbf{Z}$ & O6 Nov 91 & 20.8 & Tot. susp. solids & 5.00 & & $\mathrm{mg} / \mathrm{L}$ & Duplicate & \\
\hline $\mathbf{z}$ & 06 Nov 91 & 14.5 & Tot. susp. solids & 5.00 & & $\mathrm{mg} / \mathrm{L}$ & Duplicate & \\
\hline $\mathbf{Z}$ & 06 Nov 91 & 14.5 & Turbidity & 1.80 & & NTU & Duplicate & \\
\hline $\mathbf{G}$ & 07 Nov 91 & 20.8 & ${ }^{n} \mathrm{Cs}$ & 0.35 & 0.47 & $\mathrm{~Bq} / \mathrm{L}$ & Duplicate & \\
\hline $\mathbf{z}$ & 11 Now 91 & 20.8 & Tot. susp. solids & 5.00 & & $m g / L$ & Duplicate & \\
\hline $\mathbf{z}$ & 11 Nov 91 & 20.8 & Turbidity & 1.20 & & NTU & Duplicate & \\
\hline $\mathbf{Z}$ & 12 Nov 91 & 20.8 & Tot. susp. solids & 5.00 & & $\mathrm{mg} / \mathrm{L}$ & Duplicate & \\
\hline $\mathbf{Z}$ & 12 Nov 91 & 20.8 & Turbidity & 1.50 & & NTU & Duplicate & \\
\hline $\mathbf{z}$ & 12 Nov 91 & 14.5 & Tot. susp. solids & 5.00 & & mell & Duplicate & \\
\hline $\mathbf{z}$ & 12 Nov 91 & 14.5 & Turbidity & 1.50 & & NTU & Duplicate & \\
\hline $\mathbf{z}$ & 14 Now 91 & 20.8 & Tot. susp. solids & 5.00 & & $m g / L$ & Duplicate & \\
\hline $\mathbf{Z}$ & 14 Nov 91 & 20.8 & Turbidity & 1.10 & & NTU & Duplicate & \\
\hline $\mathbf{Z}$ & 14 Nov 91 & 14.5 & Turbidity & 1.10 & & NTU & Duplicate & \\
\hline $\mathbf{z}$ & 14 Nov 91 & 14.5 & Tot. susp. solids & 5.00 & & $\mathbf{m g} / \mathbf{L}$ & Duplicate & \\
\hline $\mathbf{Z}$ & 15 Nov 91 & 20.8 & Tot. susp. solids & 7.00 & & $\mathbf{m g} /$ & Duplicate & \\
\hline $\mathbf{G}$ & 15 Nov 91 & 20.8 & ${ }^{15} \mathrm{Cs}$ & 0.13 & 0.56 & $\mathrm{Bg} / \mathrm{L}$ & Rinse water & \\
\hline $\mathbf{Z}$ & 15 Nov 91 & 20.8 & Turbidity & 1.90 & & NTU & Duplicate & \\
\hline $\mathbf{Z}$ & 18 Nov 91 & 20.8 & Tol. susp. solids & 9.00 & & $\mathbf{m g} / \mathbf{L}$ & Duplicate & \\
\hline $\mathbf{Z}$ & 18 Nov 91 & 20.8 & Turbidity & 1.40 & & NTU & Duplicate & \\
\hline $\mathbf{Z}$ & 19 Nov 91 & 20.8 & Tot. susp. solids & 5.00 & & $\mathrm{mg} / \mathrm{L}$ & Duplicate & \\
\hline $\mathbf{z}$ & 19 Now 91 & 20.8 & Turbidity & 1.40 & & NTU & Duplicate & \\
\hline $\mathbf{z}$ & 19 Nov 91 & 14.5 & Turbidity & 1.70 & & NTU & Duplicate & \\
\hline $\mathbf{Z}$ & 19 Nov 91 & 14.5 & Tot. susp. solids & 5.00 & & $m g \Omega$ & Duplicate & \\
\hline $\mathbf{Z}$ & 20 Now 91 & 20.8 & Tot. susp. solids & 5.00 & & $\operatorname{mg} /$ & Duplicate & \\
\hline $\mathbf{z}$ & 20 Nov 91 & 20.8 & Turbidity & 1.40 & & NTU & Duplicate & \\
\hline $\mathbf{Z}$ & 21 Nov 91 & 14.5 & Tot. susp. solids & 5.00 & & $\mathbf{m g l}$ & Duplicate & \\
\hline $\mathbf{Z}$ & 21 Nov 91 & 14.5 & Turbidity & 1.50 & & NTU & Duplicate & \\
\hline $\mathbf{G}$ & 22 Nov 91 & 20.8 & ${ }^{15} \mathrm{Cs}$ & 1.10 & 0.60 & $\mathrm{~Bq} / \mathrm{L}$ & Rinse water & \\
\hline
\end{tabular}


Table E.1 (continuod)

\begin{tabular}{|c|c|c|c|c|c|c|c|c|}
\hline Sample I.D. & \multirow{2}{*}{$\frac{\text { Date }}{06 \text { Dec } 91}$} & \multirow{2}{*}{$\begin{array}{c}\begin{array}{c}\text { River } \\
\text { mile }\end{array} \\
20.8\end{array}$} & \multirow{2}{*}{$\frac{\text { Analysis }}{\text { Tot. susp. solids }}$} & \multirow{2}{*}{$\frac{\text { Result }}{30.00}$} & \multirow[t]{2}{*}{ Error } & \multirow{2}{*}{$\frac{\text { Units }}{\mathrm{mg} / \mathrm{L}}$} & \multirow{2}{*}{$\begin{array}{c}\begin{array}{c}\text { QC sample } \\
\text { type }\end{array} \\
\text { Duplicate }\end{array}$} & \multirow[t]{2}{*}{$\begin{array}{c}\text { Construction } \\
\text { phase }\end{array}$} \\
\hline $\mathbf{z}$ & & & & & & & & \\
\hline $\mathbf{Z}$ & $06 \operatorname{Dec} 91$ & 20.8 & Turbidity & 25.00 & & NTU & Duplicate & \\
\hline $\mathbf{z}$ & 06 Dec 91 & 14.5 & Tot. susp. solids & 20.00 & & $\operatorname{mg} \Omega$ & Duplicate & \\
\hline $\mathbf{z}$ & 06 Dec 91 & 14.5 & Turbidity & 21.00 & & NTU & Duplicate & \\
\hline $\mathbf{z}$ & 10 Dec 91 & 14.5 & Tot. susp. solids & 7.00 & & $\mathrm{mg} / \mathrm{L}$ & Duplicate & \\
\hline $\mathbf{z}$ & 10 Dec 91 & 14.5 & Turbidity & 4.30 & & NTU & Duplicate & \\
\hline $\mathbf{z}$ & 12 Dec 91 & 14.5 & Turbidity & 2.90 & & NTU & Duplicate & \\
\hline $\mathbf{z}$ & 12 Dec 91 & 14.5 & Tot. susp. solids & 5.00 & & $\mathrm{mg} / \mathrm{L}$ & Duplicate & \\
\hline $\mathbf{G}$ & 13 Dec 91 & 14.5 & ${ }^{17} \mathrm{Cs}$ & 0.10 & 1.10 & $\mathrm{~Bq} / \mathrm{L}$ & Rinse water & \\
\hline $\mathbf{Z}$ & 13 Dec 91 & 14.5 & Tot. susp. solids & 5.00 & & $\mathbf{m g l}$ & Duplicate & \\
\hline $\mathbf{Z}$ & 13 Dec 91 & 14.5 & Turbidity & 2.20 & & NTU & Duplicate & \\
\hline $\mathbf{z}$ & 16 Dec 91 & 14.5 & Tot. susp. solids & 6.00 & & $\mathrm{mg} / \mathrm{L}$ & Duplicate & \\
\hline $\mathbf{z}$ & $16 \operatorname{Dec} 91$ & 14.5 & Turbidity & 4.50 & & NTU & Duplicate & \\
\hline $\mathbf{Z}$ & 17 Dec 91 & 20.8 & Tot. susp. solids & 9.00 & & $\mathrm{mgll}$ & Duplicate & \\
\hline $\mathbf{z}$ & 17 Dec 91 & 20.8 & Turbidity & 8.40 & & NTU & Duplicate & \\
\hline $\mathbf{z}$ & 17 Dec 91 & 14.5 & Turbidity & 6.80 & & NTU & Duplicate & \\
\hline $\mathbf{z}$ & 17 Dec 91 & 14.5 & Tot. susp. solids & 6.00 & & $\mathrm{mg} / \mathrm{L}$ & Duplicate & \\
\hline $\mathbf{Z}$ & 18 Dec 91 & 14.5 & Tot. susp. solids & 65.00 & & $m g / L$ & Duplicate & \\
\hline $\mathbf{z}$ & 18 Dec 91 & 14.5 & Turbidity & 22.00 & & NTU & Duplicate & \\
\hline $\mathbf{z}$ & 19 nec 91 & 14.5 & Turbidity & 5.20 & & NTU & Duplicate & \\
\hline $\mathbf{z}$ & 19 Dec 91 & 14.5 & Tot. susp. solids & 9.00 & & $m g \Omega$ & Duplicate & \\
\hline $\mathbf{z}$ & 20 Dec 91 & 14.5 & Tot. susp. solids & 26.00 & & $m g /$ & Duplicate & \\
\hline $\mathbf{G}$ & 20 Dec 91 & 14.5 & ${ }^{15} \mathrm{Cs}$ & 0.27 & 0.53 & $\mathrm{~Bq} / \mathrm{L}$ & Rinse water & \\
\hline $\mathbf{Z}$ & 20 Dec 91 & 14.5 & Turbidity & 14.00 & & NTU & Duplicate & \\
\hline $\mathbf{z}$ & $26 \operatorname{Dec} 91$ & 20.8 & Turbidity & 4.10 & & NTU & Duplicate & \\
\hline $\mathbf{z}$ & 26 Dec 91 & 20.8 & Tot. susp. solids & 8.00 & & $m g / L$ & Duplicate & \\
\hline $\mathbf{z}$ & $26 \operatorname{Dec} 91$ & 14.5 & Tot. susp. solids & 7.00 & & $m g l$ & Duplicate & \\
\hline $\mathbf{z}$ & $26 \operatorname{Dec} 91$ & 14.5 & Turbidity & 4.00 & & NTU & Duplicate & \\
\hline $\mathbf{z}$ & 30 Dec 91 & 20.8 & Tot. susp. solids & 53.00 & & $\mathrm{mgl}$ & Duplicate & \\
\hline $\mathbf{Z}$ & 30 Dec 91 & 20.8 & Turbidity & 3.00 & & NTU & Duplicate & \\
\hline $\mathbf{Z}$ & 30 Dec 91 & 14.5 & Tot. susp. solids & 57.00 & & $m g / L$ & Duplicate & \\
\hline $\mathbf{Z}$ & 30 Dec 91 & 14.5 & Turbidity & 3.00 & & NTU & Duplicate & \\
\hline $\mathbf{Z}$ & 31 Dec 91 & 14.5 & Turbidity & 16.00 & & NTU & Duplicate & \\
\hline $\mathbf{z}$ & 31 Dec 91 & 14.5 & Tot. susp. solids & 39.00 & & $\mathbf{m g} / \mathbf{L}$ & Duplicate & \\
\hline $\mathbf{Z}$ & $02 \operatorname{Jan} 92$ & 14.5 & Tot. susp. solids & 27.00 & & $\mathbf{m g} / \mathrm{l}$ & Duplicate & \\
\hline
\end{tabular}


E-7

Table E1 (continued)

\begin{tabular}{|c|c|c|c|c|c|c|c|c|c|}
\hline Sample & D. & Date & $\begin{array}{l}\text { River } \\
\text { mile }\end{array}$ & Analysis & Resuli & Error & Units & $\begin{array}{c}\text { QC sample } \\
\text { type }\end{array}$ & $\begin{array}{c}\text { Construction } \\
\text { phase }\end{array}$ \\
\hline 824 & $\mathbf{z}$ & $02 \operatorname{Jan} 92$ & 14.5 & Turbidity & 8.00 & & NTU & Duplicate & \\
\hline 826 & $\mathbf{z}$ & $03 \operatorname{Jan} 92$ & 14.5 & Turbidity & 5.00 & & NTU & Duplicate & \\
\hline 826 & $\mathbf{G}$ & $03 \operatorname{Jan} 92$ & 14.5 & ${ }^{19} \mathrm{Cs}$ & -0.40 & 1.10 & $\mathrm{~Bq} / \mathrm{L}$ & Rinse water & \\
\hline 826 & $\mathbf{Z}$ & $03 \mathrm{Jan} 92$ & 14.5 & Tot. susp. solids & 13.00 & & $m g / l$ & Duplicate & \\
\hline 827 & $\mathbf{z}$ & $06 \operatorname{Jan} 92$ & 20.8 & Tot. susp. solids & 11.00 & & $m g /$ & Duplicate & \\
\hline 827 & $\mathbf{z}$ & $06 \operatorname{Jan} 92$ & 20.8 & Turbidity & 5.30 & & NTU & Duplicate & \\
\hline 828 & $\mathbf{Z}$ & $06 \operatorname{Jan} 92$ & 14.5 & Tot. susp. solids & 32.00 & ' & $\mathrm{mg} / \mathrm{L}$ & Duplicate & \\
\hline 828 & $\mathbf{Z}$ & $06 \operatorname{Jan} 92$ & 14.5 & Turbidity & 13.00 & & NTU & Duplicate & \\
\hline 831 & $\mathbf{Z}$ & $07 \operatorname{Jan} 92$ & 14.5 & Tot. susp. solids & 43.00 & & $\mathrm{mgl}$ & Duplicate & \\
\hline 831 & $\mathbf{z}$ & $07 \operatorname{Jan} 92$ & 14.5 & Turbidity & 18.00 & & NTU & Duplicate & \\
\hline 835 & $\mathbf{Z}$ & $09 \operatorname{Jan} 92$ & 14.5 & Tot. susp. solids & 26.00 & & $\mathbf{m g} / \mathbf{L}$ & Duplicate & \\
\hline 835 & $\mathbf{z}$ & $09 \operatorname{Jan} 92$ & 14.5 & Turbidity & 16.00 & & NTU & Duplicate & \\
\hline 839 & $\mathbf{z}$ & 13 Jan 92 & 14.5 & Turbidity & 53.00 & & NTU & Duplicate & \\
\hline 839 & $\mathbf{Z}$ & $13 \operatorname{Jan} 92$ & 14.5 & Tot. susp. solids & 258.00 & & $m g / L$ & Duplicate & \\
\hline 842 & $\mathbf{Z}$ & $14 \operatorname{Jan} 92$ & 14.5 & Tot. susp. solids & 122.00 & & $\mathrm{mg} / \mathrm{L}$ & Duplicate & \\
\hline 842 & $\mathbf{Z}$ & $14 \operatorname{Jan} 92$ & 14.5 & Turbidity & 30.00 & & NTU & Duplicate & \\
\hline 844 & $\mathbf{z}$ & $15 \operatorname{Jan} 92$ & 14.5 & Tot. susp. solids & 12.00 & & $\mathrm{mgl}$ & Duplicate & \\
\hline 844 & $\mathbf{Z}$ & $15 \operatorname{Jan} 92$ & 14.5 & Turbidity & 6.00 & & NTU & Duplicate & \\
\hline 846 & $\mathbf{Z}$ & $16 \operatorname{Jan} 92$ & 14.5 & Tot. susp. solids & 88.10 & & $\mathbf{m g} / \mathbf{L}$ & Duplicate & \\
\hline 846 & $\mathbf{z}$ & $16 \operatorname{Jan} 92$ & 14.5 & Turbidity & 28.00 & & NTU & Duplicate & \\
\hline 848 & $\mathbf{Z}$ & $17 \operatorname{Jan} 92$ & 14.5 & Tot. susp. solids & 10.00 & & $m g / l$ & Duplicate & \\
\hline 848 & $\mathbf{G}$ & $17 \operatorname{Jan} 92$ & 14.5 & ${ }^{151} \mathrm{Cs}$ & 0.09 & 0.55 & $\mathrm{~Bq} / \mathrm{L}$ & Rinse water & \\
\hline 848 & $\mathbf{Z}$ & $17 \operatorname{Jan} 92$ & 14.5 & Turbidity & 4.60 & & NTU & Duplicate & \\
\hline 851 & $\mathbf{Z}$ & 20 Jan 92 & 14.5 & Tot. susp. solids & 85.00 & & $\mathbf{m g l}$ & Duplicate & \\
\hline 851 & $\mathbf{Z}$ & $20 \operatorname{Jan} 92$ & 14.5 & Turbidity & 25.00 & & NTU & Duplicate & \\
\hline 855 & $\mathbf{Z}$ & $22 \operatorname{Jan} 92$ & 14.5 & Turbidity & 3.40 & & NTU & Duplicate & \\
\hline 855 & $\mathbf{Z}$ & $22 \operatorname{Jan} 92$ & 14.5 & Tot. surp. solids & 5.00 & & $\mathrm{mgl}$ & Duplicate & \\
\hline 857 & $\mathbf{Z}$ & $23 \operatorname{Jan} 92$ & 14.5 & Tot. susp. solids & 20.00 & & $\mathrm{mg} / \mathrm{L}$ & Duplicate & \\
\hline 857 & $\mathbf{z}$ & $23 \operatorname{Jan} 92$ & 14.5 & Turbidity & 10.00 & & NTU & Duplicate & \\
\hline 859 & $\mathbf{Z}$ & $24 \operatorname{Jan} 92$ & 14.5 & Tot. susp. solids & 7.00 & & $m g /$ & Duplicate & \\
\hline 859 & $\mathbf{Z}$ & $24 \operatorname{Jan} 92$ & 14.5 & Turbidity & 5.00 & & NIU & Duplicate & \\
\hline 859 & $\mathbf{G}$ & $24 \operatorname{Jan} 92$ & 14.5 & ${ }^{n} \mathrm{Cs}$ & 0.20 & 0.85 & $\mathrm{~Bq} / \mathrm{L}$ & Rinse water & \\
\hline 862 & $\mathbf{Z}$ & $27 \operatorname{Jan} 92$ & 14.5 & Turbidity & 12.00 & & NTU & Duplicate & \\
\hline 862 & $\mathbf{z}$ & $27 \operatorname{Jan} 92$ & 14.5 & Tot. susp. solids & 28.00 & & $\mathrm{mg} / \mathrm{L}$ & Duplicate & \\
\hline
\end{tabular}


Table E.1 (continuod)

\begin{tabular}{|c|c|c|c|c|c|c|c|c|}
\hline Sample I.D. & \multirow{2}{*}{$\frac{\text { Date }}{28 \text { Jan } 92}$} & \multirow{2}{*}{$\begin{array}{c}\begin{array}{c}\text { River } \\
\text { mile }\end{array} \\
14.5\end{array}$} & \multirow{2}{*}{$\frac{\text { Analysis }}{\text { Tot. susp. solids }}$} & \multirow{2}{*}{$\frac{\text { Result }}{39.00}$} & \multirow[t]{2}{*}{ Error } & \multirow{2}{*}{$\frac{\text { Units }}{\mathrm{mgl} / \mathrm{L}}$} & \multirow{2}{*}{$\begin{array}{c}\begin{array}{c}\text { QC sample } \\
\text { type }\end{array} \\
\text { Duplicate }\end{array}$} & \multirow[t]{2}{*}{$\begin{array}{c}\text { Construction } \\
\text { phase }\end{array}$} \\
\hline $\mathbf{z}$ & & & & & & & & \\
\hline $\mathbf{z}$ & $28 \operatorname{Jan} 92$ & 14.5 & Turbidity & 13.00 & & NTU & Duplicate & \\
\hline $\mathbf{z}$ & $29 \operatorname{Jan} 92$ & 14.5 & Tot. susp. solids & 5.00 & & $\mathrm{mg} / \mathrm{L}$ & Duplicate & \\
\hline $\mathbf{z}$ & $29 \operatorname{Jan} 92$ & 14.5 & Turbidity & 3.00 & & NTU & Duplicate & \\
\hline 0 & $30 \operatorname{Jan} 92$ & 14.5 & ${ }^{n} \mathrm{Cs}$ & -0.11 & 0.65 & $\mathrm{Bg} / \mathrm{L}$ & Rinse water & \\
\hline $\mathbf{G}$ & 08 Feb 92 & 20.8 & ${ }^{n} \mathrm{Cs}$ & 0.13 & 0.44 & $\mathrm{~Bq} / \mathrm{L}$ & Rinee water & \\
\hline $\mathbf{z}$ & 11 Feb 92 & 20.8 & Turbidity & 10.00 & & NTU & Duplicate & Jet grout \\
\hline $\mathbf{z}$ & 11 Feb 92 & 20.8 & Tot. susp. solids & 20.00 & & $m g /$ & Duplicate & Jet grout \\
\hline $\mathbf{z}$ & 11 Feb 92 & 20.8 & pH & 8.97 & & & Duplicate & Jet grout \\
\hline $\mathbf{z}$ & 18 Feb 92 & 20.8 & Tot. susp. solids & 6.00 & & $m g /$ & Duplicate & Jet grout \\
\hline $\mathbf{z}$ & 18 Feb 92 & 20.8 & Turbidity & 5.00 & & NTU & Duplicate & Jet grout \\
\hline $\mathbf{G}$ & 21 Feb 92 & 20.8 & ${ }^{17} \mathrm{Cs}$ & 4.70 & 0.70 & $B q / L$ & Rinse water & Jet grout \\
\hline $\mathbf{z}$ & $03 \mathrm{Mar} 92$ & 20.8 & Turbidity & 8.00 & & NTU & Duplicate & Jet grout \\
\hline $\mathbf{z}$ & 03 Mar 92 & 20.8 & Tol. susp. solids & 10.00 & & $m g / L$ & Duplicate & Jet grout \\
\hline $\mathbf{z}$ & $03 \operatorname{Mar} 92$ & 20.8 & pH & 8.19 & & & Duplicate & Jet grout \\
\hline $\mathbf{z}$ & $03 \operatorname{Mar} 92$ & 14.5 & Turbidity & 7.00 & & NTU & Duplicate & Jet grout \\
\hline $\mathbf{z}$ & 03 Mar 92 & 14.5 & $\mathrm{pH}$ & 8.26 & & & Duplicate & Jet grout \\
\hline $\mathbf{z}$ & $03 \operatorname{Mar} 92$ & 14.5 & Tot. susp. solids & 12.00 & & $m g l$ & Duplicate & Jet grout \\
\hline $\mathbf{G}$ & $06 \operatorname{Mar} 92$ & 20.8 & ${ }^{n} \mathrm{Cs}$ & 3.60 & 0.60 & $\mathrm{~B} q / \mathrm{L}$ & Rinee water & Jet grout \\
\hline $\mathbf{z}$ & $10 \operatorname{Mar} 92$ & 20.8 & pH & 8.21 & & & Duplicate & Jet grout \\
\hline $\mathbf{z}$ & 10 Mar 92 & 20.8 & ToL susp. solids & 14.00 & & $m g /$ & Duplicate & Jet grout \\
\hline $\mathbf{z}$ & $10 \mathrm{M} \times 92$ & 14.5 & Tol. susp. solids & 12.00 & & $\mathbf{m} \boldsymbol{g} /$ & Duplicste & Jet grout \\
\hline $\mathbf{z}$ & 10 Mar 92 & 14.5 & Turbidity & 5.50 & & NTU & Duplicate & Jet grout \\
\hline $\mathbf{z}$ & $10 \operatorname{Mar} 92$ & 14.5 & $\mathrm{pH}$ & 8.34 & & & Duplicate & Jet prout \\
\hline $\mathbf{z}$ & 17 Mar 92 & 14.5 & Turbidity & 5.60 & & NIU & Duplicate & Jet grout \\
\hline $\mathbf{z}$ & $17 \operatorname{Mar} 92$ & 14.5 & Tol. susp. solids & 11.00 & & $\mathrm{mg} / \mathrm{L}$ & Duplicate & Jet grout \\
\hline $\mathbf{z}$ & $17 \operatorname{Mar} 92$ & 14.5 & $\mathrm{pH}$ & 8.45 & & & Duplicate & Jet grout \\
\hline $\mathbf{z}$ & 17 Mar 92 & 14.5 & Turbidity & 5.60 & & NTU & Duplicate & Jet grout \\
\hline $\mathbf{G}$ & $20 \operatorname{Mar} 92$ & 20.8 & ${ }^{17} \mathrm{Cs}$ & 3.90 & 0.50 & $B g / L$ & Rinse water & Jet grout \\
\hline $\mathbf{z}$ & $24 \operatorname{Mar} 92$ & 20.8 & pH & 8.34 & & & Duplicate & Jet grout \\
\hline $\mathbf{z}$ & $24 \operatorname{Mar} 92$ & 20.8 & Turbidity & 9.70 & & NTU & Duplicate & Jet grout \\
\hline $\mathbf{z}$ & $24 \operatorname{Mar} 92$ & 20.8 & Tot. susp. solids & 10.00 & & $m g /$ & Duplicate & Jet grout \\
\hline $\mathbf{z}$ & $24 \operatorname{Mar} 92$ & 14.3 & $\mathrm{pH}$ & 8.35 & & & Duplicate & Jet grout \\
\hline $\mathbf{z}$ & 24 Mar 92 & 14.5 & Turbidity & 10.00 & & NTU & Duplicate & Jet grout \\
\hline $\mathbf{z}$ & $24 \operatorname{Mar} 92$ & 14.5 & Tot. susp. solids & 20.00 & & $\operatorname{mgl}$ & Duplicate & Jet grout \\
\hline
\end{tabular}


E-9

Table E1 (continued)

\begin{tabular}{|c|c|c|c|c|c|c|c|c|c|}
\hline Sample & I.D. & Date & $\begin{array}{c}\text { River } \\
\text { mile }\end{array}$ & Analysis & Result & Error & Units & $\begin{array}{l}\text { QC sample } \\
\text { type }\end{array}$ & $\begin{array}{c}\text { Construction } \\
\text { phase }\end{array}$ \\
\hline 1015 & $\mathbf{z}$ & $26 \operatorname{Mar} 92$ & 20.8 & Tot. susp. solids & 23.00 & & mg/l & Duplicate & Dredging \\
\hline 1015 & $\mathbf{z}$ & 26 Mar 92 & 20.8 & pH & 7.75 & & & Duplicate & Dredging \\
\hline 1015 & $\mathbf{z}$ & 26 Mar 92 & 20.8 & Turbidity & 10.50 & & NTU & Duplicate & Dredging \\
\hline 1016 & $\mathbf{z}$ & 26 Mar 92 & 14.5 & Turbidity & 12.50 & & NTU & Duplicate & Dredging \\
\hline 1016 & $\mathbf{z}$ & $26 \operatorname{Mar} 92$ & 14.5 & Tot. susp. solids & 34.00 & & $\mathrm{mg} / \mathrm{L}$ & Duplicate & Dredging \\
\hline 1016 & $\mathbf{z}$ & 26 Mar 92 & 14.5 & pH & 8.27 & & & Duplicate & Dredgins \\
\hline 1017 & $\mathbf{z}$ & 27 Mar 92 & 20.8 & pH & 8.29 & & & Duplicate & Dredging \\
\hline 1017 & $\mathbf{z}$ & 27 Mar 92 & 20.8 & Tot. susp. solids & 32.00 & & $\mathbf{m g l}$ & Duplicate & Dredgins \\
\hline 1017 & $\mathbf{z}$ & 27 Mar 92 & 20.8 & Turbidity & 12.40 & & NTU & Duplicate & Dredgins \\
\hline 1017 & $\mathbf{G}$ & 27 Mar 92 & 20.8 & ${ }^{17} \mathrm{Cs}$ & 13.00 & 1.00 & $\mathrm{~Bq} / \mathrm{L}$ & Rinse water & Dredging \\
\hline 1017 & $\mathbf{z}$ & 27 Mar 92 & 20.8 & $\mathrm{pH}$ & 8.23 & & & Duplicate & Dredging \\
\hline 1017 & $\mathbf{Z}$ & $27 \operatorname{Mar} 92$ & 20.8 & Turbidity & 10.50 & & NTU & Duplicate & Dredging \\
\hline 1019 & $\mathbf{Z}$ & $31 \operatorname{Mar} 92$ & 20.8 & Tot. susp. solids & 11.00 & & $\mathrm{mg} / \mathrm{L}$ & Duplicate & \\
\hline 1019 & $\mathbf{z}$ & $31 \mathrm{Mar} 92$ & 20.8 & Turbidity & 7.90 & & NTU & Duplicate & \\
\hline 1019 & $\mathbf{z}$ & $31 \operatorname{Mar} 92$ & 20.8 & pH & 8.14 & & & Duplicate & \\
\hline 1021 & $\mathbf{z}$ & 31 Mar 92 & 14.5 & $\mathbf{p H}$ & 8.30 & & & Duplicate & \\
\hline 1021 & $\mathbf{z}$ & $31 \operatorname{Mar} 92$ & 14.5 & Turbidity & 17.50 & & NTU & Duplicate & \\
\hline 1021 & $\mathbf{z}$ & 31 Mar 92 & 14.5 & Tot. susp. solids & 59.00 & & $m e l$ & Duplicate & \\
\hline 1024 & $\mathbf{Z}$ & $02 \mathrm{Apr} 92$ & 20.8 & Tot. susp. solids & 13.00 & & $\mathrm{mg} / \mathrm{L}$ & Duplicate & Dredgins \\
\hline 1024 & $\mathbf{z}$ & 02 Apr 92 & 20.8 & pH & 7.37 & & & Duplicate & Dredging \\
\hline 1024 & $\mathbf{z}$ & 02 Apr 92 & 20.8 & Turbidity & 10.00 & & NTU & Duplicate & Dredging \\
\hline 1025 & $\mathbf{z}$ & 02 Apr 92 & 14.5 & Turbidity & 3.80 & & NTU & Duplicate & Dredging \\
\hline 1025 & $\mathbf{z}$ & 02 Apr 92 & 14.5 & Tot. susp. solids & 5.00 & & $m g /$ & Duplicate & Dredgiag \\
\hline 1025 & $\mathbf{z}$ & 02 Apr 92 & 14.5 & pH & 8.28 & & & Duplicate & Dredgins \\
\hline 1026 & G & 03 Apr 92 & 20.8 & ${ }^{15} \mathrm{Cs}$ & 1.40 & 0.50 & $\mathrm{~Bq} / \mathrm{L}$ & Duplicate & Dredging \\
\hline 1027 & $\mathbf{G}$ & 03 Apr 92 & 14.5 & ${ }^{n} \mathrm{Cs}$ & $0.2 \mathrm{U}$ & 0.30 & $B g / L$ & Duplicate & Dredging \\
\hline 1028 & $\mathbf{z}$ & 07 Apr 92 & 20.8 & Tol. susp. solids & 10.00 & & $m g /$ & Duplicate & Rock anchor \\
\hline 1028 & $\mathbf{z}$ & 07 Apr 92 & 20.8 & pH & 8.28 & & & Duplicate & Rock anchor \\
\hline 1028 & $\mathbf{Z}$ & 07 Apr 92 & 20.8 & Turbidity & 9.20 & & NTU & Dupliante & Rock anchor \\
\hline 1029 & $\mathbf{z}$ & 07 Apr 92 & 14.5 & Tot. susp. solids & 35.00 & & $\mathrm{mg} /$ & Duplicate & Rock anchor \\
\hline 1029 & $\mathbf{z}$ & 07 Apr 92 & 14.5 & Turbidity & 12.50 & & NTU & Duplicate & Rock anchor \\
\hline 1029 & $\mathbf{z}$ & 07 Apr 92 & 14.5 & pH & 8.28 & & & Duplicate & Rock anchor \\
\hline 1063 & $\mathbf{G}$ & 10 Apr 92 & 20.8 & ${ }^{1 n} \mathrm{Cs}$ & 12.00 & 1.00 & $\mathrm{Bg} / \mathrm{L}$ & Rinse blank & Rock anchor \\
\hline 1064 & $\mathbf{G}$ & 10 Apr 92 & 14.5 & ${ }^{13} \mathrm{C}_{8}$ & 0.83 & 0.45 & $\mathrm{~Bq} / \mathrm{L}$ & Rince blank & Rock anchor \\
\hline
\end{tabular}


Trble E2. Project quality anurance/quality control data for surface water grab samples. Compare these values with those for Appendix D.

\begin{tabular}{|c|c|c|c|c|c|c|c|c|c|}
\hline Sample & I.D. & Date & $\begin{array}{l}\text { River } \\
\text { mile }\end{array}$ & Analyzis & Result & Error & Units & $\begin{array}{c}\text { QC sample } \\
\text { type }\end{array}$ & $\begin{array}{c}\text { Construction } \\
\text { phase }\end{array}$ \\
\hline 608 & $\mathbf{P}$ & 20 Aug 91 & 20.80 & Tot. susp. solids & 5.00 & & $m g / L$ & Rinse blank & Sheet pile \\
\hline 608 & $\mathbf{Z}$ & 20 Aug 91 & 20.80 & Turbidity & 0.21 & & NTU & Rinse blank & Sheet pile \\
\hline 608 & $\mathbf{P}$ & 20 Aug 91 & 20.80 & Tot. susp. solids & 5.00 & & $\mathrm{mg} / \mathrm{L}$ & Rinse blank & Sheet pile \\
\hline 608 & B & 20 Aug 91 & 20.80 & ${ }^{15} \mathrm{C}$ & 0.10 & 0.58 & $\mathrm{~Bq} / \mathrm{L}$ & Duplicate & Sheet pile \\
\hline 608 & $\mathbf{Z}$ & 20 Aug 91 & 20.80 & Turbidity & 1.60 & & NTU & Duplicate & Sheet pile \\
\hline 609 & $\mathbf{B}$ & 20 Aug 91 & 20.80 & ${ }^{15} \mathrm{Cs}$ & 0.25 & 0.33 & $\mathrm{Bg} / \mathrm{L}$ & Duplicate & Sheet pile \\
\hline 609 & $\mathbf{P}$ & 20 Aug 91 & 20.80 & Tot. susp. solids & 5.00 & & $\mathrm{mg} / \mathrm{L}$ & Rinse blank & Sheet pile \\
\hline 609 & $\mathbf{Z}$ & 20 Aug 91 & 20.80 & Turbidity & 1.80 & & NTU & Rinse blank & Sheet pile \\
\hline 609 & $\mathbf{P}$ & 20 Aug 91 & 20.80 & Tot. susp. solids & 5.00 & & $\mathrm{mg} / \mathrm{L}$ & Rinse blank & Sheet pile \\
\hline 609 & $\mathbf{Z}$ & 20 Aug 91 & 20.80 & Turbidity & 0.27 & & NTU & Duplicate & Sheet pile \\
\hline 611 & $\mathbf{B}$ & 21 Aug 91 & 20.80 & ${ }^{17} \mathrm{Cs}$ & 0.05 & 0.43 & $\mathrm{~Bq} / \mathrm{L}$ & Rinse blank & Sheet pile \\
\hline 612 & $\mathbf{B}$ & 21 Aug 91 & 20.80 & ${ }^{19} \mathrm{Cs}$ & 0.05 & 0.43 & $\mathrm{~Bq} / \mathrm{L}$ & Rinse blank & Sheet pile \\
\hline 686 & $\mathbf{Z}$ & 30 Sep 91 & 20.80 & Turbidity & 1.70 & & NTU & Duplicate & Rock sirmor \\
\hline 686 & $\mathbf{Z}$ & $30 \operatorname{Sep} 91$ & 20.80 & Tot. susp. solids & 5.00 & & $m g / L$ & Duplicate & Rock armor \\
\hline 953 & $\mathbf{Z}$ & 02 Mar 92 & 20.80 & Turbidity & 1250 & & NTU & Duplicate & Jet grout \\
\hline 953 & $\mathbf{Z}$ & 02 Mar 92 & 20.80 & Tot. susp. solids & 27.00 & & $m g / L$ & Duplicate & Jet grout \\
\hline 976 & $\mathbf{Z}$ & 09 Mar 92 & 20.80 & pH & 7.42 & & & Rinse blank & Jet grout \\
\hline 976 & $\mathbf{Z}$ & 09 Mar 92 & 20.80 & Turbidity & 0.29 & & NTU & Rinse blank & Jet grout \\
\hline 977 & $\mathbf{z}$ & 09 Mar 92 & 20.80 & Turbidity & 0.21 & & NTU & Rinse blank & Jet grout \\
\hline 977 & $\mathbf{B}$ & $09 \operatorname{Mar} 92$ & 20.80 & ${ }^{15} \mathrm{C}$ & 0.30 & 2.00 & $\mathrm{~Bq} / \mathrm{L}$ & Rinse blank & Jet grout \\
\hline 977 & $\mathbf{Z}$ & 09 Mar 92 & 20.80 & pH & 7.33 & & & Rinse blank & Jet grout \\
\hline 979 & $\mathbf{Z}$ & 10 Mar 92 & 20.80 & pH & 7.94 & & & Duplicate & \\
\hline 979 & B & $10 \mathrm{Mar} 92$ & 20.80 & ${ }^{19} \mathrm{C}$ & 8.60 & 2.00 & $\mathrm{Bg} / \mathrm{L}$ & Duplicate & \\
\hline 979 & $\mathbf{Z}$ & $10 \operatorname{Mar} 92$ & 20.80 & Turbidity & 24.00 & & NTU & Duplicate & \\
\hline 981 & $\mathbf{z}$ & 10 Mar 92 & 20.80 & pH & 6.88 & & & Rinse blank & Jet grout \\
\hline 981 & $\mathbf{z}$ & $10 \operatorname{Mar} 92$ & 20.80 & Turbidity & 0.47 & & NTU & Rinse blank & Jet grout \\
\hline 1002 & $\mathbf{z}$ & $11 \mathrm{Mar} 92$ & 20.80 & pH & 7.47 & & & Duplicate & Jet grout \\
\hline 1002 & $\mathbf{Z}$ & 11 Mar 92 & 20.80 & Turbidity & 27.00 & & NTU & Duplicate & Jet grout \\
\hline 1002 & $\mathbf{B}$ & $11 \operatorname{Mar} 92$ & 20.80 & ${ }^{n} \mathrm{C}$ & 11.00 & 2.00 & $\mathrm{Bg} / \mathrm{L}$ & Duplicate & Jet grout \\
\hline 1002 & $\mathbf{Z}$ & $11 \operatorname{Mar} 92$ & 20.80 & $\mathrm{pH}$ & 7.74 & & & Duplicate & Jet grout \\
\hline 1002 & $\mathbf{Z}$ & 11 Mar 92 & 20.80 & Turbidity & 0.40 & & NTU & Duplicate & Jet grout \\
\hline 1002 & $\mathbf{B}$ & 11 Mar 92 & 20.80 & ${ }^{15} \mathrm{C}$ & 11.00 & 2.00 & $\mathrm{~Bq} / \mathrm{L}$ & Duplicate & Jet grout \\
\hline 1004 & $\mathbf{Z}$ & $12 \operatorname{Mar} 92$ & 20.80 & Turbidity & 29.00 & & NTU & Rinse blank & Jet grout \\
\hline 1004 & $\mathbf{z}$ & $12 \operatorname{Mar} 92$ & 20.80 & Turbidity & 0.22 & & NTU & Rinse blank & Jet grout \\
\hline 1004 & $\mathbf{Z}$ & $12 \operatorname{Mar} 92$ & 20.80 & pH & 7.24 & & & Rinse blank & Jet groul \\
\hline
\end{tabular}


E-11

Table E2 (continued)

\begin{tabular}{|c|c|c|c|c|c|c|c|c|c|}
\hline Sample & I.D. & Date & $\begin{array}{l}\text { River } \\
\text { mile }\end{array}$ & Analysis & Result & Error & Units & $\begin{array}{l}\text { QC sample } \\
\text { type }\end{array}$ & $\begin{array}{c}\text { Construction } \\
\text { phase }\end{array}$ \\
\hline 1006 & $\mathbf{Z}$ & $16 \operatorname{Mar} 92$ & 20.80 & Turbidity & 3.90 & & NTU & Duplicate & Jet grout \\
\hline 1006 & B & 16 Mar 92 & 20.80 & ${ }^{15} \mathrm{Cs}$ & 0.60 & 2.20 & $\mathrm{~Bq} / \mathrm{L}$ & Duplicate & Jet grout \\
\hline 1006 & $\mathbf{z}$ & $16 \operatorname{Mar} 92$ & 20.80 & pH & 8.81 & & & Duplicate & Jet grout \\
\hline 1006 & B & $16 \operatorname{Mar} 92$ & 20.80 & ${ }^{19} \mathrm{Cs}$ & -0.60 & 2.20 & $\mathrm{Bg} / \mathrm{L}$ & Duplicate & Jet groul \\
\hline 1007 & $\mathbf{z}$ & 16 Mar 92 & 20.80 & pH & 7.81 & & & Rinse blank & Jet grout \\
\hline 1007 & $\mathbf{z}$ & $16 \operatorname{Mar} 92$ & 20.80 & Turbidity & 0.20 & & NTU & Rinse blank & Jet grout \\
\hline 1034 & B & $25 \operatorname{Mar} 92$ & 20.80 & ${ }^{17} \mathrm{Cs}$ & 1.50 & 2.90 & $B q / L$ & Duplicate & Jet grout \\
\hline 1034 & B & 25 Mar 92 & 20.80 & ${ }^{n} \mathrm{Cs}$ & 1.50 & 2.90 & $\mathrm{~Bq} / \mathrm{L}$ & Duplicate & Jet grout \\
\hline 1077 & $\mathbf{Z}$ & 08 Apr 92 & 20.79 & Turbidity & 2.30 & & NTU & Duplicate & Drilling \\
\hline 1077 & $\mathbf{Z}$ & 08 Apr 92 & 20.79 & $\mathrm{pH}$ & 8.58 & & & Duplicate & Drilling \\
\hline 1081 & $\mathbf{Z}$ & 08 Apr 92 & 20.80 & pH & 7.78 & & & Rinse blank & Drilling \\
\hline 1081 & $\mathbf{Z}$ & 08 Apr 92 & 20.80 & Turbidity & 0.26 & & NTU & Rinse blank & Drilling \\
\hline
\end{tabular}


ORNL/ER-150

\section{DISTRIBUTION}

1. F. D. Adams

2. L. D. Bates

3. D. T. Bell

4. B. G. Blaylock

5. H. L. Boston

6. G. F. Cada

7. J. B. Cannon

8. R. B. Cook

9. J. H. Cushman

10. M. F. P. DeLozier

11. N. W. Durfee

12. J. T. Etheridge

13-17. C. J. Ford

18. D. E. Fowler

19. M. L. Frank

20. S. B. Garland

21. C. W. Gehrs

22. C. D. Goins

23. P. J. Halsey

24. S. G. Hildebrand

25. F. O. Hoffman

26. R. O. Hultgren

27. K. G. Kahl

28. P. Kanciruk

29. B. L. Kimmel

30. T. M. Koepp

31. F. C. Kornegay
32. E. H. Krieg

33. J. R. Lawson

34-36. D. M. Matteo

37. J. R. Merriman

38-39. P. T. Owen

40. F. S. Patton

41. D. E. Reichle

42. M. W. Rosenthal

43. T. H. Row

44. G. E. Rymer

45. F. E. Sharples

46. D. S. Shriner

47. S. H. Stow

48. R. R. Turner

49. S. D. Van Hoesen

50. R. I. Van Hook

51. D. Watkins

52-54. M. T. Wefer

55. R. K. White

56. R. Williams

57. P. S. Wood

58. Central Research Library

59-61. ESD Library

62. Clinch River Program DMC

63-67. ER Document Management Center

68. ORNL Laboratory Records

69. ORNL Patent Section

70. Office of Assistant Manager for Energy Research and Development, Department of Energy Oak Ridge Field Office, P.O. Box 2001, Oak Ridge, Tennessee 37831-8600

71-73. J. Bilyeu, Department of Energy, Office of Environmental Restoration, Eastern Area D\&D Branch, EM-423 (GTN), Washington, DC 20545

74-78. M. Ferre, Department of Energy Oak Ridge Field Office, P. O. Box 2001, Oak Ridge, TN 37831-8541

79. J. F. Franklin, Bloedel Professor of Ecosystem Analysis, College of Forest Resources, University of Washington, Anderson Hall AR-10, Seattle, WA 98195

80. C. S. Gist, Department of Energy Oak Ridge Field Office, P.O. Box 2001, Oak Ridge, TN 37831-8541

81. R. C. Harriss, Institute for the Study of Earth, Oceans, and Space, Science and Engineering Research Building, University of New Hampshire, Durham, NH 03824

82. G. Y. Jordy, Director, Office of Program Analysis, Office of Energy Research, ER-30, G-226, U.S. Department of Energy, Washington, DC 20545

83. M. Leslie, CDM Federal Programs, 800 Oak Ridge Turnpike, Oak Ridge, TN 37830

84. R. L Nace, Branch Chief, N tenrichment Facilities, Oak Ridge Program Division, Office of Eastern Area Programs, Office of Environmental Restoration, EM-423, Trevion 2, U.S. Department of Energy, Washington, DC 20585

85. R. H. Olson, Professor, Microbiology and Immunology Department, University of Michigan, Medical Sciences II, \#5605, 1301 East Catherine Street, Ann Arbor, MI 48109-0620 
86-88. D. G. Page, Department of Energy Oak Ridge Field Office, P.O. Box 2001, Oak Ridge, TN 37831-8541

89. A. Patrinos, Director, Environmental Sciences Division, Office of Health and Environmental Research, Office of Energy Research, ER-74, U.S. Department of Energy, Washington, DC 20585

90-91. R. C. Sleeman, Department of Energy Oak Ridge Field Office, P.O. Box 2001, Oak Ridge, TN 37831-8541

92-93. J. T. Sweeney, Department of Energy Oak Ridge Field Office, P.O. Box 2001, Oak Ridge, TN 37831-8541

94. D. W. Swindle, Radian Corporation, 120 South Jefferson Circle, Oak Ridge, TN 37830

95. H. M. Thron, Chief, Enrichment Facilities, Oak Ridge Program Division, Office of Eastern Area Programs, Office of Environmental Restoration, EM-423, Trevion 2, U.S. Department of Energy, Washington, DC 20585

96. F. J. Wobber, Environmental Sciences Division, Office of Health and Environmental Research, Office of Energy Research, ER-74, U.S. Department of Energy, Washington, DC 20585

97-98. Office of Scientific and Technical Information, P.O. Box 62, Oak Ridge, TN 37831 

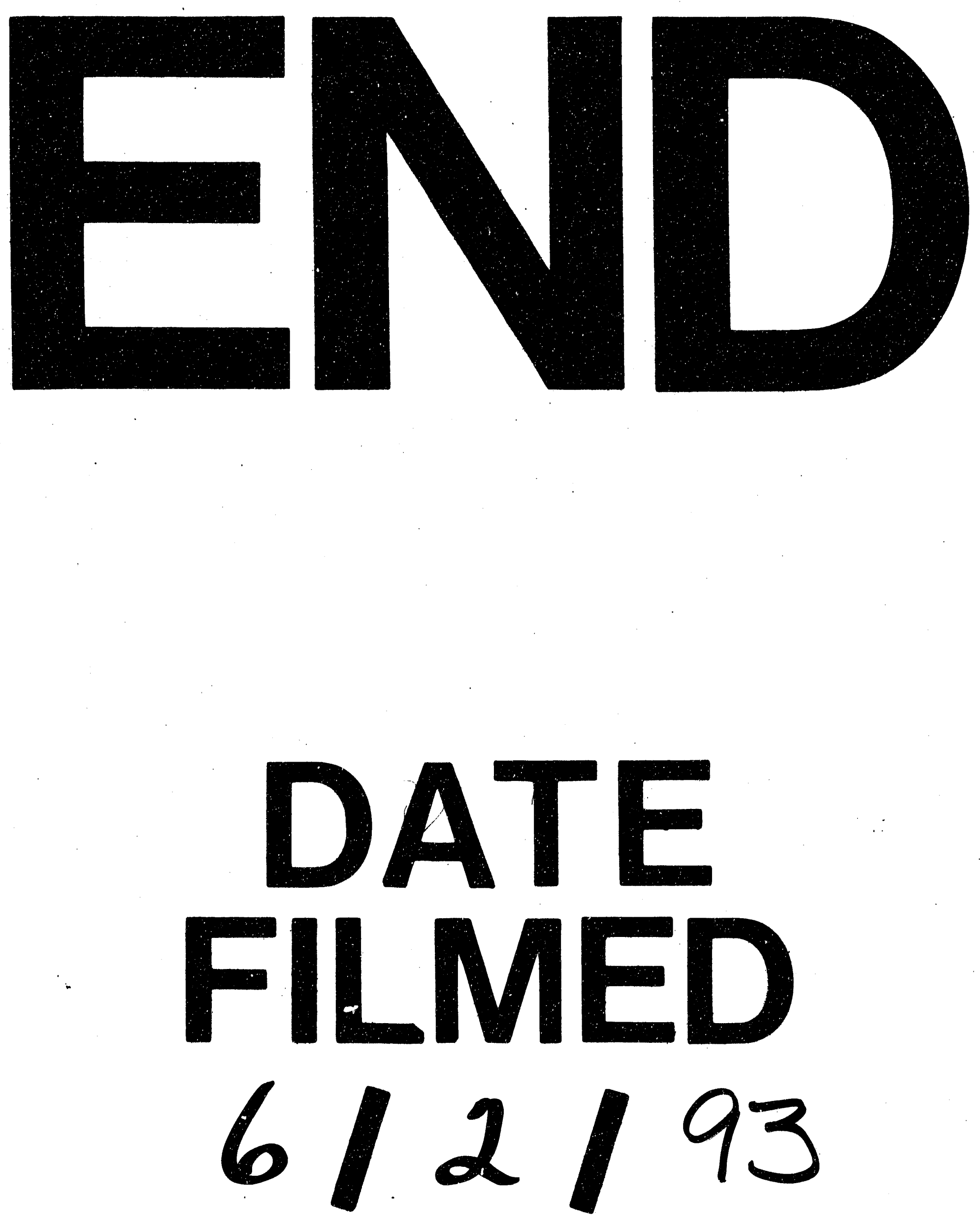
